\title{
Small mammals of the Estação Ecológica de Bananal, southeastern Atlantic Forest, Brazil, with description of a new species of Brucepattersonius (Rodentia, Sigmodontinae)
}

\author{
Edson Fiedler de Abreu-Júnior ${ }^{1,2}$ \& Alexandre Reis Percequillo ${ }^{1,3}$
}

\footnotetext{
1 Universidade de São Paulo (USP), Escola Superior de Agricultura “Luiz de Queiroz” (ESALQ), Departamento de Ciências Biológicas (LCB), Laboratório de Mamíferos; Programa de Pós-Graduação Interunidades em Ecologia Aplicada (PPGI-EA) - Universidade de São Paulo (USP), Escola Superior de Agricultura "Luiz de Queiroz" (ESALQ), Centro de Energia Nuclear na Agricultura (CENA). Piracicaba, São Paulo, Brasil.

${ }^{2}$ ORCID: http://orcid.org/0000-0003-4547-6160. E-mail: edson.abreu@usp.br (corresponding author)

${ }^{3}$ ORCID: http://orcid.org/0000-0002-7892-8912. E-mail: percequillo@usp.br
}

\begin{abstract}
The Atlantic Forest harbors an impressive diversity of nonvolant small mammals. Despite having been the target of many ecological and taxonomical studies in the last decades, this group is still poorly known in several aspects - basic information on intrapopulational morphologic variation and proper diagnoses are lacking for many species, even for common taxa. This is related to the fact that large series of specimens from a single locality are extremely rare in scientific collections. A consistent sampling effort was conducted at Estação Ecológica de Bananal, northeastern São Paulo State, throughout seven field expeditions between 2003 and 2011, under the coordination of the Museu de Zoologia da Universidade de São Paulo, resulting in extensive series of nonvolant small mammals. We studied in detail the external and cranio-dental morphology of this sample (575 specimens), that we assigned to 31 known species besides one new species to science. Most species belong to order Rodentia (26 species) from the families (ricetidae (22 species) and Echimyidae (four species); the remaining (six species) are marsupials from the order Didelphimorphia, family Didelphidae. This is one of the most diverse assemblages ever recorded in this biome, and includes some of the rarest endemic rodents of the Atlantic Forest, such as Abrawayaomys ruschii, Blarinomys breviceps, Drymoreomys albimaculatus, Phaenomys ferrugineus, Phyllomys kerri and Rhagomys rufescens. This outstanding richness can be explained by the extensive sampling effort performed for a long period of time. Also, the capture success of the third expedition (8.25\%) is among the highest rates obtained in the Atlantic Forest and the extensive use of pitfall traps was fundamental for trapping this high number of species. Herein, we provide an annotated list of nonvolant small mammals occurring at Estação Ecológica de Bananal, with comprehensive analyses of morphological variation and detailed diagnoses that will allow their proper identification, and will provide a sound basis for a better comprehension of the geographic variation of these taxa across the biome. We also present a formal description for a new species of Brucepattersonius from the mountain ranges along the Paraíba do Sul valley. For five species of sigmodontine rodents, we investigate the levels of ontogenetic and sexual variation, and while the first is accentuate in all analyzed species the second does not contribute significantly to intrapopulation variation.
\end{abstract}

Key-Words. Annotated list; Rodentia; Didelphimorphia; Intrapopulational variation; Sexual dimorphism.

Resumo. A Floresta Atlântica abriga uma diversidade impressionante de pequenos mamíferos não voadores que, apesar de terem sido alvo de inúmeros estudos taxonómicos e ecológicos nas últimas décadas, ainda carecem de informações básicas de variação intrapopulacional e diagnoses detalhadas, incluindo para algumas das espécies mais comuns. Isto está relacionado ao fato de que séries numerosas de espécimes de uma mesma localidade são extremamente raras em coleções científicas. Um consistente esforço amostral foi conduzido na Estação Ecológica de Bananal, nordeste do Estado de São Paulo, em sete expedições de campo realizadas entre os anos de 2003 e 2011, sob coordenação do Museu de Zoologia da Universidade de São Paulo, resultando em extensas séries de pequenos mamíferos não voadores. Nos estudamos em detalhe a morfologia externa e crânio-dental destas amostras (575 espécimes) e identificamos 31 espécies conhecidas, além de uma nova espécie para a ciência. A maioria das espécies são roedores (Rodentia; 26 espécies) das famílias Cricetidae (22 espécies) e Echimyidae (quatro espécies); as demais (seis espécies) são marsupiais (Didelphimorphia) da família Didelphidae. A assembleia de pequenos mamíferos da Estação Ecológica de Bananal é uma das mais diversas já registradas no bioma, que inclui algumas das espécies endêmicas mais raras da Floresta Atlântica, como Abrawayaomys ruschii, Blarinomys breviceps, Drymoreomys albimaculatus, Phaenomys ferrugineus, Phyllomys kerri e Rhagomys rufescens. Esta riqueza ímpar pode ser explicada pelo extenso esforço 
amostral realizado em um longo período de tempo. Além disso, o sucesso de captura registrado para a terceira expedição (8,25\%) está entre os mais altos obtidos na Floresta Atlântica e a utilização de armadilhadas de interceptação e queda foi determinantes para a captura de tantas e raras espécies. Neste estudo, apresentamos uma lista comentada dos pequenos mamíferos não-voadores que ocorrem na Estação Ecológica de Bananal, com análises detalhadas de variação morfológica e diagnoses detalhadas, que serão úteis para a identificação destas espécies e ainda fornecerão base para análises de variação geográfica destes táxons ao longo do bioma. Apresentamos também a descrição formal de uma nova espécie de Brucepattersonius que ocorre em áreas montanhosas ao logo do vale do Rio Paraíba do Sul. Para cinco espécies de roedores sigmodontíneos, nos investigamos a influência das variações ontogenética e sexual e concluímos que, enquanto a primeira é muito acentuada em todas as espécies estudadas, a segunda não corresponde a uma fonte significativa de variação.

Palavras-Chave. Lista comentada; Rodentia; Didelphimorphia; Variação intrapopulacional; Dimorfismo sexual.

\section{INTRODUCTION}

The Atlantic Forest (AF) is one of the most important biomes for biodiversity conservation in the world (Myers et al., 2000). Originally, it occupied $15 \%$ of the Brazilian territory along the Atlantic coast, encompassing 17 states from the northern State of Rio Grande do Norte to the southern State of Rio Grande do Sul (Fundação SOS Mata Atlântica \& INPE, 2013). Today, about $85 \%$ of its original area has been reduced in Brazil (Ribeiro et al., 2009), and the AF became the second most threatened biome in the world (Campanili \& Prochnow, 2006). The AF domain includes primarily Dense and Open Ombrophilous Forests (embracing high-montane, montane, sub-montane and lowland rainforests), Mixed Ombrophilous Forest and Semideciduous and Deciduous Seasonal Forests, as well as associated ecosystems and transitional areas (Câmara, 2005). This heterogeneous complexity of vegetation and the geographical characteristics associated with a large latitudinal and altitudinal range in the AF have been suggested as determinant factors to its notorious biological diversity and endemism (Ribeiro et al., 2009).

Around 300 species of mammals occur in the AF and about $30 \%$ of them are endemic (Costa et al., 2000; Paglia et al., 2012). This impressive diversity is not equally distributed among orders, as small sized species ( $<1 \mathrm{~kg}$ sensu Bovendorp et al., 2017) from the orders Rodentia and Didelphimorphia (traditionally regarded as nonvolant small mammals) account for over a third of the AF mammal's richness, with 124 species (Bovendorp et al., 2017). This number, however, is still growing, as new species and genera are continuously being described (e.g., Percequillo et al., 2011; Tavares et al., 2011; Gonçalves \& Oliveira, 2014) and species thought to be very rare or extinct in the AF are being rediscovered (e.g., Passamani et al., 2011; Cerboncini et al., 2014; Abreu-Júnior et al., 2018).

Despite the new findings involving nonvolant small mammals in the $A F$, there are several aspects of the group that are poorly studied. For instance, basic information on intrapopulational morphologic variation is not available for many species. Additionally, proper diagnoses are lacking for most species, even for the commonest taxa. This is related to the fact that large series of specimens from a single locality are extremely rare in scientific collections. A consistent sampling effort was conducted at Estação Ecológica de Bananal (EEB), northeastern São Paulo State, in 2003, 2004 and 2011 under the coordination of the Museu de Zoologia da Universidade de São Paulo (MZUSP), resulting in extensive series of nonvolant small mammals. These samples allowed us to study in detail the external and cranio-dental morphology of 32 species occurring at EEB, one of the largest assemblages recorded in this biome (see Bovendorp et al., 2017; Figueiredo et al., 2017). Herein, we provide an annotated list of these species, with comprehensive analyses of morphological variation and detailed diagnoses, that will allow their proper identification, and will provide a sound basis for a better comprehension of the geographic variation of these taxa across the biome. We also present the description of a new species of the genus Brucepattersonius, from the valley of the Rio Paraíba do Sul. For five species of sigmodontine rodents (one Akodontini, one Oryzomyini, one Wiedomyini, and two incertae sedis), we also investigated the levels of ontogenetic and sexual variation. Moreover, we compared the efficiency of conventional (Sherman, Tomahawk and Museum Special) and pitfall traps in capturing nonvolant small mammals at EEB.

\section{MATERIAL AND METHODS}

\section{Sampling}

The EEB comprises an area of 884 hectares in the municipality of Bananal, northeastern São Paulo State, Brazil, between $22^{\circ} 15^{\prime}$ to $22^{\circ} 37^{\prime} \mathrm{S}$ and $44^{\circ} 07^{\prime}$ to $44^{\circ} 22^{\prime} \mathrm{W}$ (Fig. 1 ). The EEB is located in the Serra da Bocaina - region of sharp relief inserted in the Serra do Mar mountain range (da Silva \& Castelletti, 2003). The elevation at EEB ranges from 1,100 to 2,000 $\mathrm{m}$ above sea level (Castro, 2001). The climate is characterized as mesothermal, with mild summers and without dry season. The average annual rainfall ranges between 1,500 and 2,000 $\mathrm{mm}$ (Castro, 2001). The mean annual temperature ranges between $20^{\circ}$ and $33^{\circ} \mathrm{C}$; the maximum absolute temperature varies from $36^{\circ}$ to $38^{\circ} \mathrm{C}$ and the minimum absolute temperature varies from $0^{\circ}$ to $4^{\circ} \mathrm{C}$ (Fundação Florestal, 2013). The vegetation at EEB is typical of montane (between 1,100 $\mathrm{m}$ and $1,500 \mathrm{~m}$ ) and high-montane formations (above 1,500 $\mathrm{m}$ ) of the Dense Ombrophilous Forest, according to the classification of Veloso et al. (1991). According to Baitello et al. (1998), 75\% of the EEB is formed by primary forest, where the forest canopy can reach $30 \mathrm{~m}$ high. 


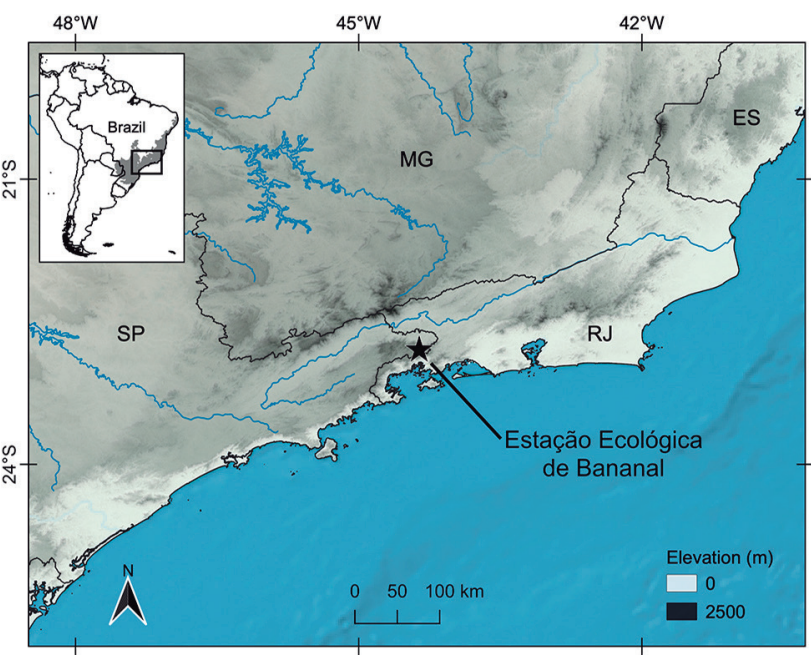

Figure 1. Location of the Estação Ecológica de Bananal (EEB) in the Serra da Bocaina mountain chain, municipality of Bananal, São Paulo State, Brazil. SP: São Paulo State, MG: Minas Gerais State, RJ: Rio de Janeiro State, ES: Espírito Santo State.

Sampling of nonvolant small mammals was conducted at EEB under the coordination of MZUSP during seven field expeditions: one expedition in December, 2003 (mammal sampling was conducted by A.R. Percequillo, E. Hingst-Zaher, C. Ayres and L. Geise); five expeditions in January, April, May, September and December, 2004 (mammal sampling was performed by the MZUSP herpetological team and EEB staff, coordinated by H. Zaher); and one expedition in December, 2011 (mammal sampling was conducted by E.F. Abreu-Júnior). Specimens were trapped with conventional (Sherman, Tomahawk and Museum Special) and pitfall (36 L buckets) traps. The sampling effort was recorded only for three expeditions, as follows: in December 2003, nonvolant small mammals were trapped for 17 consecutive days with a sampling effort of 3,266 conventional-trap nights and 2,984 pitfall-trap nights; in April 2004, the field expedition lasted 12 days and the sampling effort of conventional traps was 400 trap nights and of pitfall traps was 2,400 trap nights; in December 2011, nonvolant small mammals were trapped for three days only and the sampling effort of the conventional traps was 270 trap nights and of the pitfall traps was 126 trap nights.

All specimens collected at EEB are housed at MZUSP. However, a significant portion of the material had not been incorporated in the collection and, for that reason, the specimens in question have not received the museum number. In such cases, specimens are referred by their field numbers preceded by the acronyms EEB or EBM. All collecting and preparation procedures followed the guidelines of the Animal Care and Use Committee of the American Society of Mammalogists (Sikes \& The Animal Care and Use Committee, 2016).

\section{Species identification}

Species occurring at EEB were identified through morphological and molecular analyses. We analyzed qualitative and quantitative traits of external and cranio-dental morphology (as will be explained in details in the following sections) and compared these information with literature data and with specimens from other localities of the AF, particularly those from southeastern Brazil, deposited in the following collections: American Museum of Natural History (AMNH), New York, U.S.A.; Coleção de Mamíferos da Escola Superior de Agricultura "Luiz de Queiroz", Universidade de São Paulo (LMUSP), Piracicaba, Brazil; Coleção de Mamíferos da Universidade Federal de Lavras (CMUFLA), Lavras, Brazil; Museu Nacional da Universidade Federal do Rio de Janeiro (MN) Rio de Janeiro, Brazil; Museu de Zoologia da Universidade de São Paulo (MZUSP), São Paulo, Brazil; Museum of Vertebrate Zoology, University of California (MVZ), Berkeley, U.S.A.; Smithsonian National Museum of Natural History (USNM), Washington, D.C., U.S.A. Also, we examined specimens from smaller collections, those of Dr. Ana Paula Carmignotto (APC), housed at Universidade Federal de São Carlos (UFSCAR), Brazil, and Dr. Renata Pardini, which specimens were collected by Adriana Bueno $(A B)$ and are housed at Laboratório de Diversidade e Conservação de Mamíferos da Universidade de São Paulo (USP), Brazil. Molecular analyses were carried out for taxonomically complex genera - i.e., those with poorly defined and/or cryptic species - such as: Marmosops, Akodon, Brucepattersonius, Oxymycterus, Oligorzyzomys, Phyllomys and Trinomys. We sequenced the mitochondrial cytochrome $b$ (CYTB) as a barcode gene to confirm the species identity. Results of the molecular analysis are not shown, as they are beyond the scope of this paper, except for the genus Brucepattersonius, as these results results provided taxonomic novelties.

\section{Morphological quantitative analyses}

Quantitative analyzes were performed on two sets of variables: body measurements and cranio-dental measurements. Body variables represent dimensions of the external morphology of specimens that were taken before preparation; they are annotated in field catalogues and specimen tags and are as follows: total length (TTL); head and body length (HBL); tail length (TL); hind foot length, not considering the claws (HF1); hind foot length, considering the claws (HF2); ear length (EL); and weight (W). The data from body variables are presented for each species as absolute values or descriptive statistics (mean, standard deviation, maximum and minimum values, and sample size), when more than three specimens were available. Data from two variables ( $\mathrm{HBL}$ and $\mathrm{W}$ ) were also employed to delimit body-size classes for Sigmodontinae rodents. We generated a scatterplot with HBL values on " $X$ " axis and $W$ values on " $Y$ " axis, looking for the formation of discontinuities and/or clusters that could be interpreted as discrete categories. For most species, our dataset for this analysis was from EEB specimens; however, for six species (Abrawayaomys ruschii, Drymoreomys albimaculatus, Juliomys pictipes, Oxymycterus dasytrichus, Phaenomys ferrugineus and Rhagomys rufescens) body 
measurements were not available for adult specimens from EEB and we used literature data.

The cranio-dental variables were measured with a $0.01 \mathrm{~mm}$ precision digital caliper and were defined independently for each of the families of small mammals (Didelphidae, Cricetidae and Echimyidae). A total of 19 cranio-dental measurements (Fig. 2) were defined to characterize the didelphid marsupials. These variables were based on Mustrangi \& Patton (1997), Patton et al. (2000), Voss et al. (2001), Voss et al. (2004) and Rossi et al.
(2010): Rostral width (RW), width of the rostrum at the level of canines; Nasal breadth (NB), breadth of nasals at the maxillary-frontal suture; Least interorbital breadth (LIB), measured at the narrowest point across the frontals between the orbits, anterior to the postorbital processes if present; Postorbital constriction (POC), measured at the narrowest point across the frontals between the temporal fossae, posterior to the postorbital processes if present; Zygomatic breadth (ZB), greatest breadth across the zygomatic arches; Braincase breadth (BB), greatest
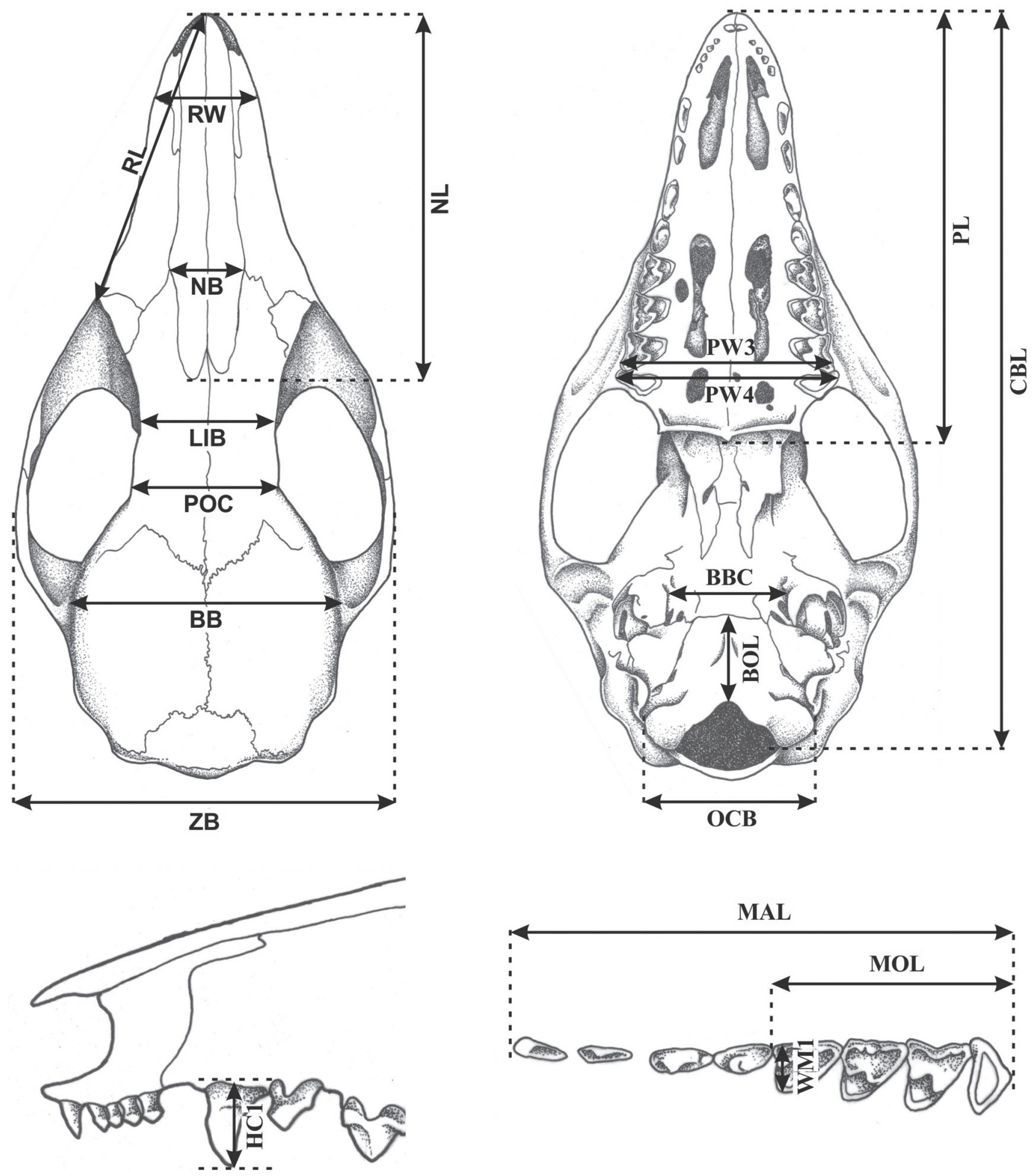

MAL

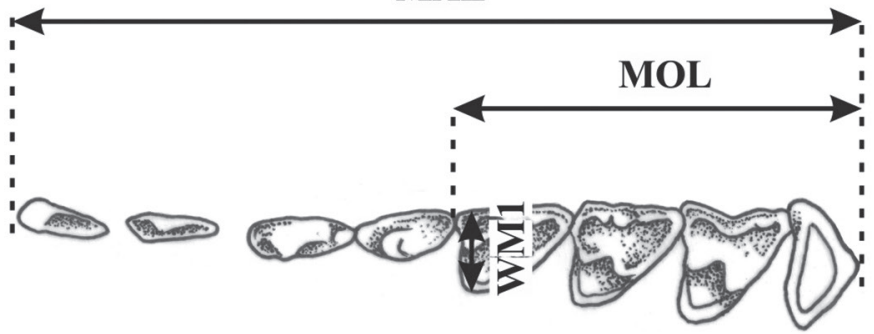

Figure 2. Skull of Marmosops paulensis (MZUSP 32512) showing the 19 cranio-dental morphometric variables used for didelphid marsupials. 
breadth of braincase above the squamosal root of the zygomatic arch; Nasal length (NL), midline distance from anterior tip to posterior margins of nasals; Rostral length $(R L)$, measured from the anterior margin of the orbit to the midline tip of the nasal bones; Condylobasal length $(\mathrm{CBL})$, measured from the occipital condyles to the anteriormost point of the premaxillae; Palatal length (PL), midline distance from the anteriormost point of the premaxillae to the posterior margin of the hard palate; Maxillary toothrow length (MAL), measured from the anterior face of the canine to the posterior margin of M4; Molar toothrow length (MOL), measured on the labial margin of the toothrow from M1 to M4; Height of upper canine $(\mathrm{HC} 1)$, measured from the alveolar margin to the apex of $\mathrm{C} 1$; Width of first upper molar (WM1), measured from the labial margin of the crown to the lingual apex of the protocone of M1; Palatal width (PW3), width of palate taken across the labial margins of third molars; Palatal width (PW4), width of palate taken across the labial margins of fourth molars; Breadth of basicranium (BBC), least distance between the anteromedial margins of the right and left alisphenoid tympanic processes; Basioccipital length (BOL), distance from anterior margin of foramen magnum to basioccipital-basisphenoid suture; Occipital condyle breadth (OCB), breadth across the outside margins of the occipital condyles. The age classification of didelphid marsupials followed Voss et al. (2001).

For cricetid rodents, 25 cranio-dental measurements (Fig. 3) were based on Voss (1988), Musser et al. (1998), Percequillo (1998) and Percequillo (2003), as follows: Greatest skull length (GSL), measured from the anteriormost projection of nasals to posteriormost projection of occipital region on midline of skull; Condylo-incisive length $(\mathrm{CIL})$, measured from the greater curvature of one upper incisor to the articular surface of the occipital condyle; Condylo-zigomatic length (CZL), measured from the zygomatic notch to the articular surface of the occipital condyle; Length of diastema (LD), from the crown of the first upper molar to the lesser curvature of the upper incisor; Length of incisive foramen (LIF), greatest internal anterior-posterior dimension of incisive foramen; Breadth of incisive foramina (BIF), greatest dimension measured across the internal surface of both incisive foramina; Length of molars (LM), from the anterior surface of $\mathrm{M} 1$ to posterior margin of M3; Breadth of $\mathrm{M} 1$ (BM1), greatest crown breadth of the M1 across the paracone-protocone; Incisive depth (ID), depth of the upper incisors taken from the anterior surface to the posterior surface of one upper incisor; Incisive width (IW), width of upper incisors taken across the lateral margins of both incisors; Length of nasals (LN), greatest anterior-posterior dimension of one nasal bone; Breadth of rostrum (BR), greatest dimension measured across the external border of the nasolacrimal capsules; Rostral length $(\mathrm{RL})$, measured from the anteriormost part of one nasal to the anteriormost internal curvature of orbital fossa; Zygomatic breadth (ZB), greatest dimension across the squamosal root of zygomatic arches; Breadth of zygomatic plate (BZP), measured across the central area of zygomatic plate; Least interorbital breadth (LIB), least distance across the frontal bones; Orbital fossa length (OFL), greatest length of the orbital fossa between the squamosal and maxillary roots of the zygomatic arch; Palatal length $(\mathrm{PL})$, midline distance from the posterior margin of 11 to the anterior border of mesopterygoid fossa; Length of palatal bridge (LPB), measured from the posterior border of the incisive foramen to the anterior border of the mesopterygoid fossa; Breadth of bony palate (BBP), measured across the hard palate on the lingual surface of first upper molars crowns; Height of braincase $(\mathrm{HBC})$, vertical distance from the sphenoccipital suture to the top of braincase; Breadth of braincase (BBC), greatest width of braincase measured posterior to the squamosal root of zygomatic arches; Interparietal breadth (IPB), greatest width of interparietal measured across the most distant lateral points; Interparietal length (IPL), greatest distance between the anterior and posterior margins of interparietal; Occipital condyle breadth (OCB), breadth across the outside margins of the occipital condyles. The criteria used to classify cricetid rodents into age groups were the eruption and wear of the occlusal surface of the molars, following Voss (1991), Brandt \& Pessôa (1994) and Percequillo (1998), according to which five distinct classes were applied.

For echimyid rodents, 23 cranio-dental measurements (Fig. 4) were defined and modified based on Patton et al. (2000), Leite (2003) and lack-Ximenes (2005), as follows: Greatest skull length (GSL), measured from the anteriormost projection of nasals to posteriormost projection of occipital region on midline of skull; Condylo-incisive length (CIL), measured from the greater curvature of one upper incisor to the articular surface of the occipital condyle; Length of diastema (LD), from the crown of the upper premolar to the lesser curvature of the upper incisor; Maxillary toothrow length (MAL), measured from the anterior face of PM4 to the posterior margin of M3; Breadth of PM4 (BPM), greatest crown breadth of the PM4; Length of incisive foramen (LIF), greatest internal anterior-posterior dimension of incisive foramen; Breadth of incisive foramina (BIF), greatest dimension measured across the internal surface of both incisive foramina; Palatal length (PL), midline distance from the posterior margin of I1 to the anterior border of mesopterygoid fossa; Length of nasals (LN), greatest anterior-posterior dimension of one nasal bone; Rostral length $(R L)$, measured from the anteriormost part of one nasal to the anteriormost internal curvature of orbital fossa; Orbital fossa length (OFL), greatest length of the orbital fossa between the squamosal and maxillary roots of the zygomatic arch; Breadth of rostrum (BR), greatest dimension measured across the external border of the nasolacrimal capsules; Least interorbital breadth (LIB), least distance across the frontal bones; Zygomatic breadth (ZB), greatest dimension across the squamosal root of zygomatic arches; Mastoid breadth (MB), distance across cranium at mastoid processes; Palatal width (PW), width of palate taken across the labial margins of third molars; Distance between bullae (DBB), least distance between tympanic bullae taken at basioccipital-basisphenoid suture; Occipital condyle breadth (OCB), breadth across the 

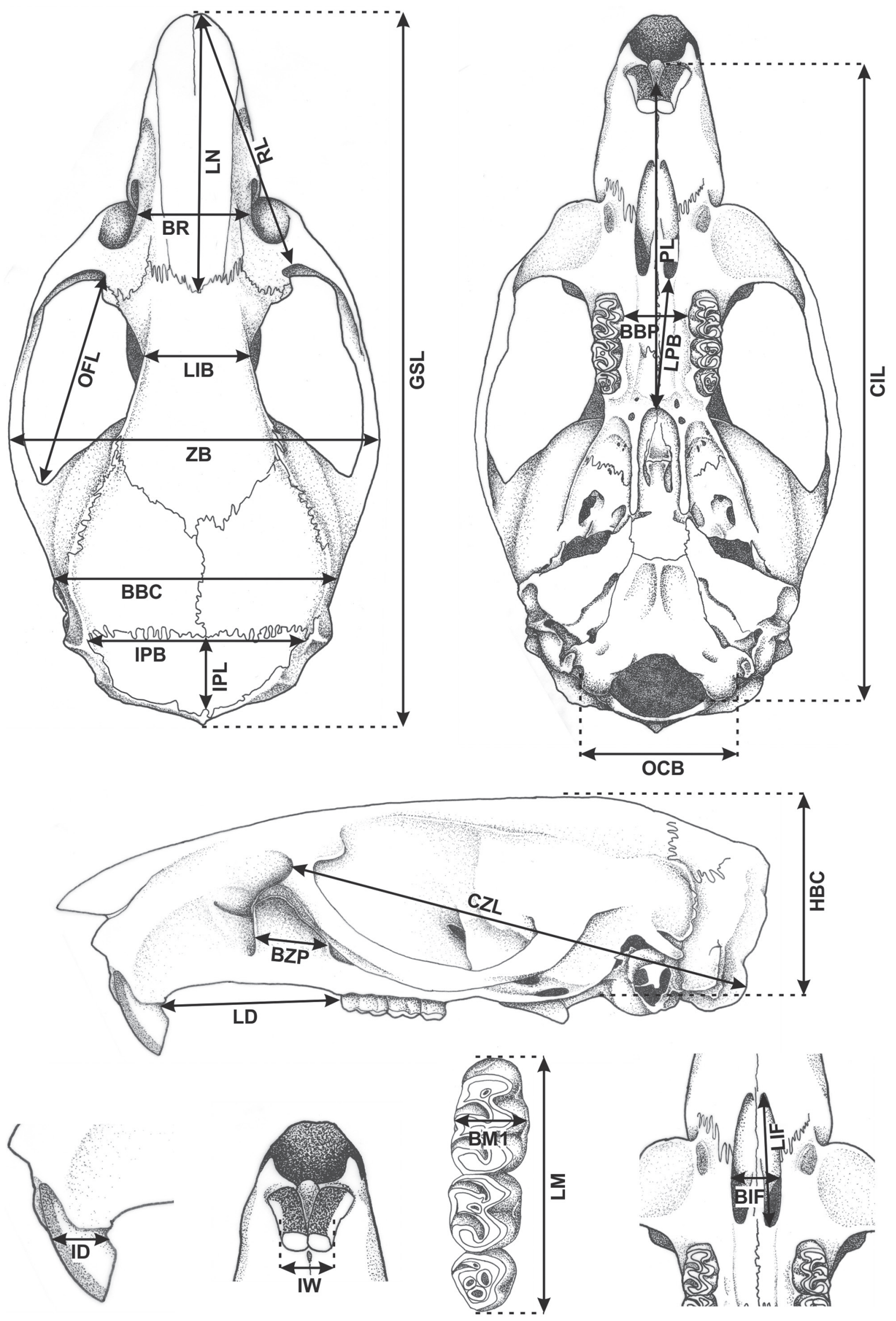

Figure 3. Skull of Euryoryzomys russatus (MZUSP 28392) showing the 25 cranio-dental morphometric variables used for cricetid rodents. 

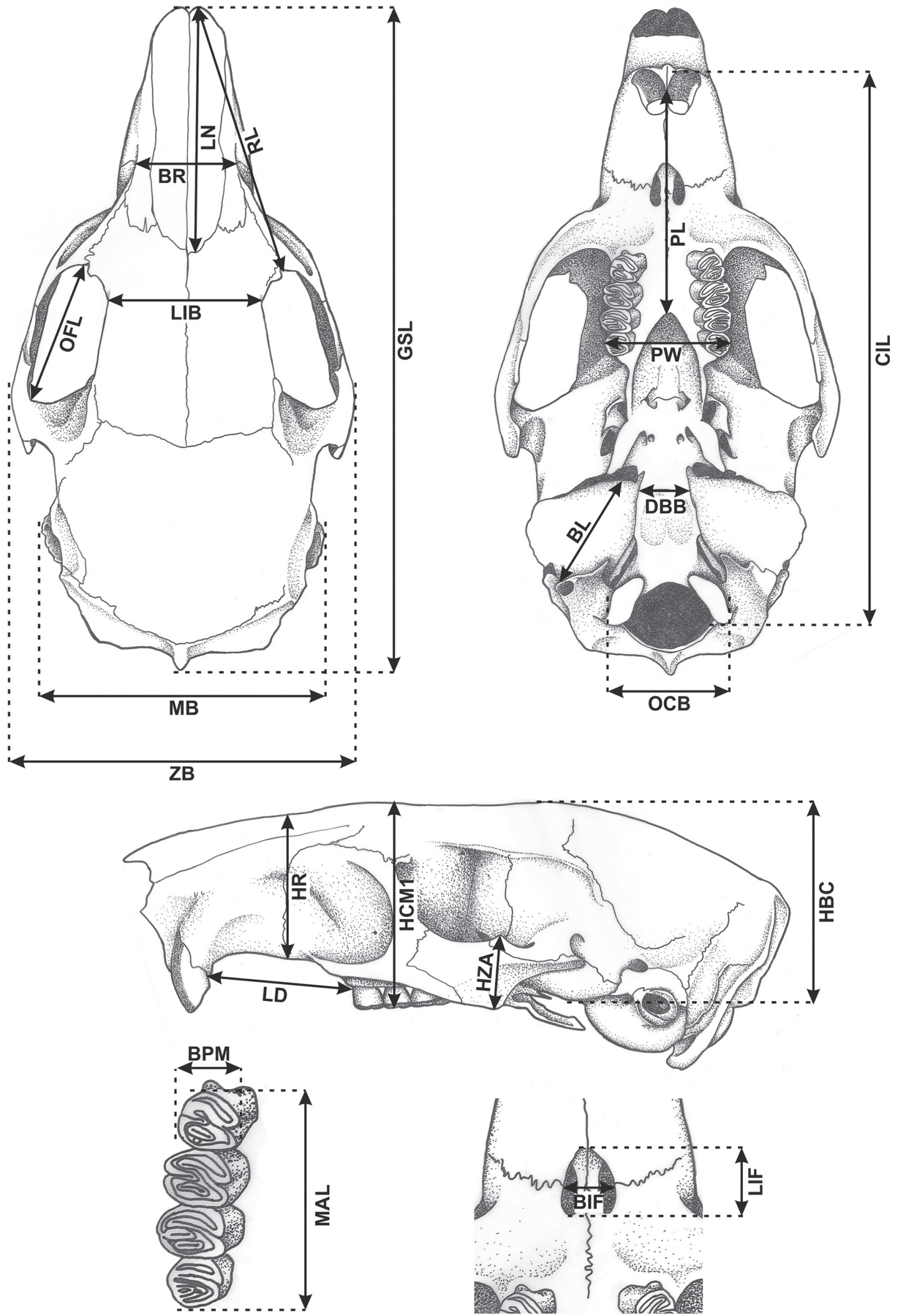

Figure 4. Skull of Trinomys dimidiatus (EEB 1013) showing the 23 cranio-dental morphometric variables used for echimid rodents. 
outside margins of the occipital condyles; Bullar length $(\mathrm{BL})$, greatest distance from anterior to posterior margins of tympanic bullae; Height of braincase ( $\mathrm{HBC})$, vertical distance from the spheno-occipital suture to the top of braincase; Height of cranium at M1 (HCM1), vertical distance from the occlusal surface of $M 1$ to the top of the cranium; Height of rostrum (HR), height of rostral region taken at premaxillary-maxillary suture; Height of zygomatic arch (HZA), greatest height of zygomatic arch including the postorbital process of zygoma. The age classes of echimid rodents were established based on the eruption and wear of ridges and folds of the upper molariform teeth following Leite (2003) and lack-Ximenes (2005). Besides descriptive statistics, data obtained from cranio-dental variables were used in intra-population variation analyses (differences in age and sex).

\section{Ontogenetic and sexual variation}

We tested ontogenetic and sexual variation in five species - Akodon montensis, Oligoryzomys nigripes, Juliomys ossitenuis, Delomys dorsalis and D. sublineatus - which presented the largest sample sizes. The normality of each sample (series of specimens per variable per species) was verified using the Kolmogorov-Smirnov test (Vanzolini, 1993; Sokal \& Rohlf, 1981). After that, ontogenetic variation among the pre-defined age classes was tested using the Analysis of Variance (ANOVA) followed by the Tukey post-hoc test. Next, the Student t-test was performed to evaluate sexual dimorphism, individually in the age-classes or in groups of classes, as determined by the previous test of ontogenetic variation (Brandt \& Pessôa, 1994; Camardella et al., 1998; Prado \& Percequillo, 2011). In addition to the tests described above, data from cranio-dental variables were used to differentiate among species in four genera (Akodon, Brucepattersonius, Oligoryzomys and Delomys), using principal component analysis (PCA) based on a covariant matrix (Neff \& Marcus, 1980; Johnson \& Wichern, 1999; Manly, 2005), and exploratory bivariate graphics. All statistical analyzes were conducted on SPSS Statistics 17.0 (SPSS Inc., 2008).

\section{Morphological qualitative analyses}

We analyzed qualitative characters of external and cranio-dental morphology. Characters of external morphology were examined in dry (skins) and wet (70\% alcohol) preserved specimens; approximately 30 characters were chosen, based on previous studies on rodents and marsupials (e.g., Percequillo, 1998; Leite, 2003; lack-Ximenes, 2005; Weksler, 2006; Voss \& Jansa, 2009). Cranio-dental characters were defined for each family independently. For Cricetidae we followed: Hershkovitz (1962), Carleton (1980), Voss (1988), Carleton \& Musser (1989), Myers et al. (1990), Voss (1993), Musser et al. (1998), Percequillo (1998), Hershkovitz (1998), Christoff et al. (2000), Weksler (2006), Costa et al. (2007) and Machado et al. (2011). For Echimyidae: Leite (2003) and lack-Ximenes (2005). And for Didelphidae: Mustrangi \& Patton (1997) and Voss \& Jansa (2003, 2009). In total, ca. 50 cranio-dental characters were analyzed in cricetid rodents, 30 in echimid rodents and 30 in didelphid marsupials. It is important to note that characters of the dental morphology for cricetid rodents are restricted to the first upper (M1) and lower (m1) molars, considering that they offer a greater number of diagnostic features. The results of the qualitative analysis were used to compose the diagnoses and descriptions of species. The frequency at which certain character states occur in the population was provided in every case that polymorphism was detected. When appropriate, we comment on changes in the frequency of character states that are associated with age categories.

\section{Molecular analyses}

Total DNA was extracted from muscle or liver tissue using DNeasy Blood \& Tissue kit (Qiagen Inc.) following the manufactures' protocol. We amplified the CYTB gene by PCR using the external primers MVZ05 (Smith \& Patton, 1993) and UMMZ04 (Jansa et al., 1999). For Brucepattersonius samples we also used internal primers: BRUCE793R (5' ATTCTGGTTTAATGTGTGCTGG) and BRUCE664F (5' CTGATAAAATTCCATTCCACC). PCR reactions and cycle conditions were similar to described in Percequillo et al. (2017). PCR products were purified using ExoSap-IT (Affymetrix) and sequenced using amplification primers and BigDye Terminator (Life Technologies Corp.), on ABI3130 sequencer (Applied Biosystems). Sequences were edited using Geneious 6.1.4 (Biomatters Ltd.) and multiple alignments were performed using MUSCLE (Edgar, 2004) implemented on Geneious. Best fitting nucleotide substitution models were searched in jModelTest 2.1.2 (Darriba et al., 2012; Guindon \& Gascuel, 2003) and chosen following the Bayesian Information Criterion (BIC).

Phylogenetic reconstructions were generated using Maximum Likelihood (ML) and Bayesian Inference (BI) methods. ML analyses were performed through 20 independent searches in RaxML 7.0.3 (Stamatakis, 2006); nodal support was obtained by 1,000 bootstraps replicates. $\mathrm{BI}$ analyses were performed by $5 \times 10^{6}$ generations of MCMC (Markov Chain Monte Carlo), using MrBayes 3.2.0 (Ronquist et al., 2012); nodal support was obtained as posterior probability. The first $25 \%$ of BI trees were discarded as burn-in. The results of MCMC runs were inspected in Tracer 1.5 (Rambaut \& Drummond, 2007). Trees were edited in FigTree 1.4.0 (Rambaut, 2012). Mean sequence divergences ( $p$-distances) within and among species were obtained using Mega 6.0 (Tamura et al., 2013).

\section{RESULTS AND DISCUSSION}

We recognized 32 species of nonvolant small mammals occurring at EEB based on the examination of 575 specimens collected between December 2003 and December 2011. Most species belong to order Rodentia 
Table 1. Sampling effort (trap nights; E), sampling success (S), number of specimens captured $(N)$ and number of species recorded $(R)$ in each field expedition to EEB. The results are showed separated by method (pitfall traps and conventional traps) and in total. Data not available (NA).

\begin{tabular}{|c|c|c|c|c|}
\hline Expeditions & Metrics & Pitfall traps & Conventional traps & Total \\
\hline \multirow{4}{*}{ First } & $E$ & 2,984 & 3,266 & 6,250 \\
\hline & $S$ & $5.06 \%$ & $2.08 \%$ & $3.50 \%$ \\
\hline & N & 151 & 68 & 219 \\
\hline & $\mathrm{R}$ & 18 & 13 & 22 \\
\hline \multirow{4}{*}{ Second } & $E$ & NA & NA & NA \\
\hline & $S$ & NA & NA & NA \\
\hline & $\mathrm{N}$ & 16 & 0 & 16 \\
\hline & $\mathrm{R}$ & 8 & 0 & 8 \\
\hline \multirow{4}{*}{ Third } & $E$ & 2,400 & 400 & 2,800 \\
\hline & S & $9.37 \%$ & $1.50 \%$ & $8.25 \%$ \\
\hline & $\mathrm{N}$ & 225 & 6 & 231 \\
\hline & $\mathrm{R}$ & 17 & 3 & 17 \\
\hline \multirow{4}{*}{ Fourth } & $E$ & NA & NA & NA \\
\hline & $S$ & NA & NA & NA \\
\hline & N & 43 & 0 & 43 \\
\hline & $\mathrm{R}$ & 13 & 0 & 13 \\
\hline \multirow{4}{*}{ Fifth } & $E$ & NA & NA & NA \\
\hline & S & NA & NA & NA \\
\hline & $\mathrm{N}$ & 6 & 0 & 6 \\
\hline & $\mathrm{R}$ & 3 & 0 & 3 \\
\hline \multirow{4}{*}{ Sixth } & $E$ & NA & NA & NA \\
\hline & $S$ & NA & NA & NA \\
\hline & $\mathrm{N}$ & 32 & 0 & 32 \\
\hline & R & 11 & 0 & 11 \\
\hline \multirow{4}{*}{ Seventh } & $E$ & 126 & 270 & 396 \\
\hline & $S$ & $8.73 \%$ & $4.44 \%$ & $5.80 \%$ \\
\hline & $\mathrm{N}$ & 11 & 12 & 23 \\
\hline & $\mathrm{R}$ & 7 & 5 & 9 \\
\hline
\end{tabular}

(26 species) from the families Cricetidae (22 species) and Echimyidae (four species); the remaining (six species) are marsupials from the order Didelphimorphia, family Didelphidae. Table 1 summarizes the number of specimens and species collected in each field expedition separated by capture method. For the first, third and seventh expeditions, we also present the sampling effort and capture success. Information on sampling effort for the remaining expeditions was not available. The third expedition yielded the greatest number of specimens collected and the best overall capture rate $(8.25 \%)$; the first expedition, on the other hand, yielded the smallest capture rate $(3.5 \%)$, but provided the highest species richness per expedition (22 species). In the seventh expedition, the sampling effort was the lowest, but capture success was intermediate $(5.8 \%)$.

Comparing the performance of pitfall traps versus conventional traps (Sherman, Tomahawk and Museum Special), pitfall traps captured more specimens and had better capture success in all expeditions in which both methods were used (first, third and seventh). Nine out of 22 species recorded during the first expedition were captured exclusively by pitfall traps (see Table 2). In the second expedition, pitfall trap effort was much greater than conventional trap effort, and the former type of trap was responsible for capturing 14 out of 17 species
Table 2. Nonvolant small mammals sampled at EEB and respective method(s) of capture of each species in each field expedition. Conventional trap (C), Pitfall trap (P).

\begin{tabular}{|c|c|c|c|c|c|c|c|}
\hline \multirow[b]{2}{*}{ Taxa } & \multicolumn{7}{|c|}{ Expeditions } \\
\hline & 产 & $\begin{array}{l}\text { ర్ } \\
\text { ڤ్ }\end{array}$ & 홀 & $\begin{array}{l}\text { 돌 } \\
\text { 호 }\end{array}$ & 恶 & 吾 & 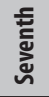 \\
\hline \multicolumn{8}{|l|}{ DIDELPHIMORPHIA } \\
\hline \multicolumn{8}{|l|}{ Didelphidae } \\
\hline Gracilinanus microtarsus & - & - & $P$ & $P$ & $P$ & - & - \\
\hline Didelphis aurita & $C$ & - & - & - & - & - & - \\
\hline Marmosops paulensis & $C, P$ & - & $P$ & - & $P$ & - & $C, P$ \\
\hline Monodelphis iheringi & $P$ & - & $P$ & - & - & - & - \\
\hline Monodelphis scalops & $C$ & - & $P$ & $P$ & - & - & - \\
\hline Philander quica & $C$ & 一 & 一 & 一 & 一 & 一 & - \\
\hline \multicolumn{8}{|l|}{ RODENTIA } \\
\hline \multicolumn{8}{|l|}{ Cricetidae } \\
\hline Abrawayaomys ruschii & $P$ & - & - & $P$ & - & - & - \\
\hline Akodon cursor & $C$ & - & - & - & - & - & - \\
\hline Akodon montensis & $C, P$ & - & $C, P$ & $P$ & - & $P$ & $C, P$ \\
\hline Blarinomys breviceps & $P$ & - & - & - & $P$ & - & - \\
\hline Brucepattersonius soricinus & $P$ & - & $P$ & - & - & $P$ & - \\
\hline Brucepattersonius n. sp. & $P$ & - & $P$ & - & - & - & - \\
\hline Castoria angustidens & $P$ & - & $C, P$ & $P$ & - & - & - \\
\hline Delomys dorsalis & $C, P$ & - & $C, P$ & - & - & $P$ & - \\
\hline Delomys sublineatus & $C, P$ & $P$ & $P$ & $P$ & - & $P$ & $C, P$ \\
\hline Drymoreomys albimaculatus & $P$ & - & - & - & - & $P$ & - \\
\hline Euryoryzomys russatus & - & - & - & - & - & - & $C$ \\
\hline Juliomys ossitenuis & $C, P$ & $P$ & $P$ & $P$ & - & $P$ & $P$ \\
\hline Juliomys pictipes & - & - & $P$ & - & - & $P$ & - \\
\hline Nectomys squamipes & - & $P$ & - & - & - & - & - \\
\hline Oligoryzomys flavescens & $P$ & - & $P$ & - & - & - & $P$ \\
\hline Oligoryzomys nigripes & $C, P$ & - & $P$ & $P$ & - & $P$ & $P$ \\
\hline Oxymycterus dasytrichus & - & $P$ & - & $P$ & - & - & - \\
\hline Phaenomys ferrugineus & - & - & $P$ & - & - & - & - \\
\hline Rhagomys rufescens & - & - & - & $P$ & - & - & - \\
\hline Rhipidomys itoan & $C, P$ & $P$ & $P$ & $P$ & - & $P$ & - \\
\hline Sooretamys angouya & $C, P$ & $P$ & $P$ & - & - & $P$ & - \\
\hline Thaptomys nigrita & $C, P$ & $P$ & - & $P$ & - & $P$ & $P$ \\
\hline \multicolumn{8}{|l|}{ Echimyidae } \\
\hline Euryzygomatomys spinosus & - & - & - & $P$ & - & - & - \\
\hline Phyllomys kerry & $P$ & - & - & - & - & - & - \\
\hline Phyllomys sulinus & - & $P$ & - & - & - & - & - \\
\hline Trinomys dimidiatus & - & - & - & - & - & 一 & C \\
\hline Total number of species & 22 & 8 & 17 & 13 & 3 & 11 & 9 \\
\hline Species sampled with both methods & 9 & 0 & 3 & 0 & 0 & 0 & 3 \\
\hline Only pitfall traps & 9 & 8 & 14 & 13 & 3 & 11 & 4 \\
\hline Only conventional traps & 4 & 0 & 0 & 0 & 0 & 0 & 2 \\
\hline
\end{tabular}

in that expedition. In the seventh expedition, of the nine species sampled, four were collected with pitfall traps exclusively.

In the following pages, we present the morphological identification of each species based exclusively on the specimens collected at EEB. For seven genera (Monodelphis, Akodon, Brucepattersonius, Oligoryzomys, Juliomys, Delomys and Phyllomys) we present a discussion on the morphological traits useful for the species identification of its congeners, and for five species (Akodon montensis, Oligoryzomys nigripes, Juliomys ossitenuis, Delomys dorsalis and $D$. sublineatus) we also present analyses of 
ontogenetic and sexual variation, which is useful to understand the influence of these sources of variation in the species delimitations. Moreover, we present a formal description of a new species of Brucepattersonius from EEB and five other highland localities in the southeastern $A F$, based on molecular and morphologic evidences.

\section{Species accounts}

\section{Order Didelphimorphia Family Didelphidae Subfamily Didelphinae Tribe Didelphini}

\section{Didelphis aurita Wied-Neuwied 1826}

Voucher material: $\mathrm{n}=1$; male: uncatalogued: EEB 663 .

Specimen identification: Only one specimen of $D$. aurita was collected at EEB and only its skin was found at MZUSP. Therefore, the cranio-dental description is not presented for this species.

Very large size $(\mathrm{TTL}=806.0 \mathrm{~mm}$; HF1 $=57.0 \mathrm{~mm}$; HF2 = $61.0 \mathrm{~mm} ; E L=50.0 \mathrm{~mm} ; \mathrm{W}=2000 \mathrm{~g}$ ). Head pelage mostly blackish with whitish spots on snout, cheeks and superciliary region. Dorsal pelage dense, moderately soft and long; aristiforms reaching $80 \mathrm{~mm}$ length; very heterogeneous coloration, predominantly whitish with black spots particularly on the dorsal midline, general aspect grizzled; individual hairs with basal portion cream white, a central portion black and an apical portion white. Lateral pelage also very heterogeneous, predominantly cream white with black spots and sprinkled of pure white; limit between lateral and ventral color weak. Pelage of arms and legs black sprinkled of white, contrasting with dorsal and ventral pelage. Ventral pelage mostly cream, with grayish and blackish spots concentrated near the ventral midline and the throat region. Mystacial vibrissae long, reaching the base of the pinnae when laid back. Pinnae large and rounded, blackish colored; covered with very short and sparse hairs, generally black. Dorsal surface of manus covered by short hairs, black colored; digits with long claws, claw of $\mathrm{d} I \mathrm{II}=6.20 \mathrm{~mm}$. Pes dorsally covered by short hairs, black colored; dIII similar in length to dII and IV; digits with long claws, claw of dIII $=8.30 \mathrm{~mm}$; tufts of ungual hairs very sparse and short, not extending beyond the claws, dark colored. Tail longer than head and body; covered at base (from $1 / 5$ to $1 / 6$ ) with similar pelage of dorsum and after that covered by short hairs; usually three hairs with same size (two scales longer) emerging from distal margin of each scale, blackish colored; scales large, distinguishable to the naked eye, with spiral arrangement; tail particolored, basal half black on both sides, with black hairs, and apical half whitish on both sides, with white hairs.

Field observations: The only specimen of $D$. aurita collected at EEB was an adult male. It was captured with a Tomahawk trap in December, 2003.

\section{Philander quica (Temminck, 1824)}

Voucher material: $\mathrm{n}=1$; male: MZUSP 32516.

Specimen identification: Large size. Head pelage dark gray, almost black, contrasting strongly with dorsal pelage; presence of a whitish gray spot on each side of superciliary region. Dorsal pelage dense and soft; predominantly homogeneous coloration, gray slightly brownish sprinkled with light gray. Lateral pelage gray, lighter than dorsum, sprinkled with very light gray; limit between lateral and ventral color very clear. Ventral pelage predominantly yellowish cream, slightly grayish cream on abdominal and throat regions; individual hairs entirely cream or with gray base (from $1 / 3$ to $1 / 2$ ) on the abdominal and throat regions. Mystacial vibrissae long, reaching the base of pinnae when laid back but not surpassing the top of them. Pinnae large and oval in shape, dark colored on the sides and whitish on central portion; covered with short and very sparse hairs, whitish colored. Dorsal surface of manus covered by short hairs, grayish colored; digits with short claws, claw of dIII $=3.5 \mathrm{~mm}$. Pes dorsally covered by short hairs, grayish colored; dIII similar in length to dII and IV; digits with medium claws, claw of dIII = $3.80 \mathrm{~mm}$; tufts of ungual hairs very sparse and short, not extending beyond the claws, whitish colored. Tail longer than head and body $(\approx 138 \%$ of head and body length; $n=1$ ); covered at base (from $1 / 4$ to $1 / 5$ ) with similar pelage of dorsum and posteriorly covered by short hairs; usually four hairs with same size (two scales longer) emerging from distal margin of each scale; scales large, distinguishable to the naked eye, with spiral arrangement; tail particolored, basal portion (approximately $2 / 3$ ) dark brown colored in the dorsal surface, with dark brown hairs, and brown slightly lighter in the venter, with hairs brown at base and whitish at top, and apical potion of the tail (approximately $1 / 3$ ) unpigmented, with whitish hairs in both faces.

Skull slender and elongated (Fig. 5). Premaxillary rostral process absent; premaxillae not extending beyond the alveolar margin of the first upper incisor (I1). Nasals short, not extending anteriorly above 11, exposing nasal orifice in dorsal view; nasals conspicuously widened posteriorly than anteriorly; posterior margins of nasals convergent. Interorbital region slight posteriorly convergent, with supraorbital margins rounded. Postorbital processes present but very reduced. Sagittal crest weakly developed in the juvenile specimen of EEB, but usually well developed on adult specimens. Interparietal present. One lacrimal foramen usually present on each side of skull, exposed on lateral view. Parietal and alisphenoid in contact on lateral braincase. Paroccipital process large. Incisive foramina short, not surpassing the canine teeth (C1). Premaxillary-maxillary suture collinear to superior canine alveolus. Maxillopalatine fenestrae present; right and left fenestrae divided by a medial septum. Maxillary fenestrae present but small. Palatine fenestrae present and large. Posterolateral palatal foramina present with complete ossified margins. Transverse canal foramen absent. Extracranial course of mandibular nerve enclosed 


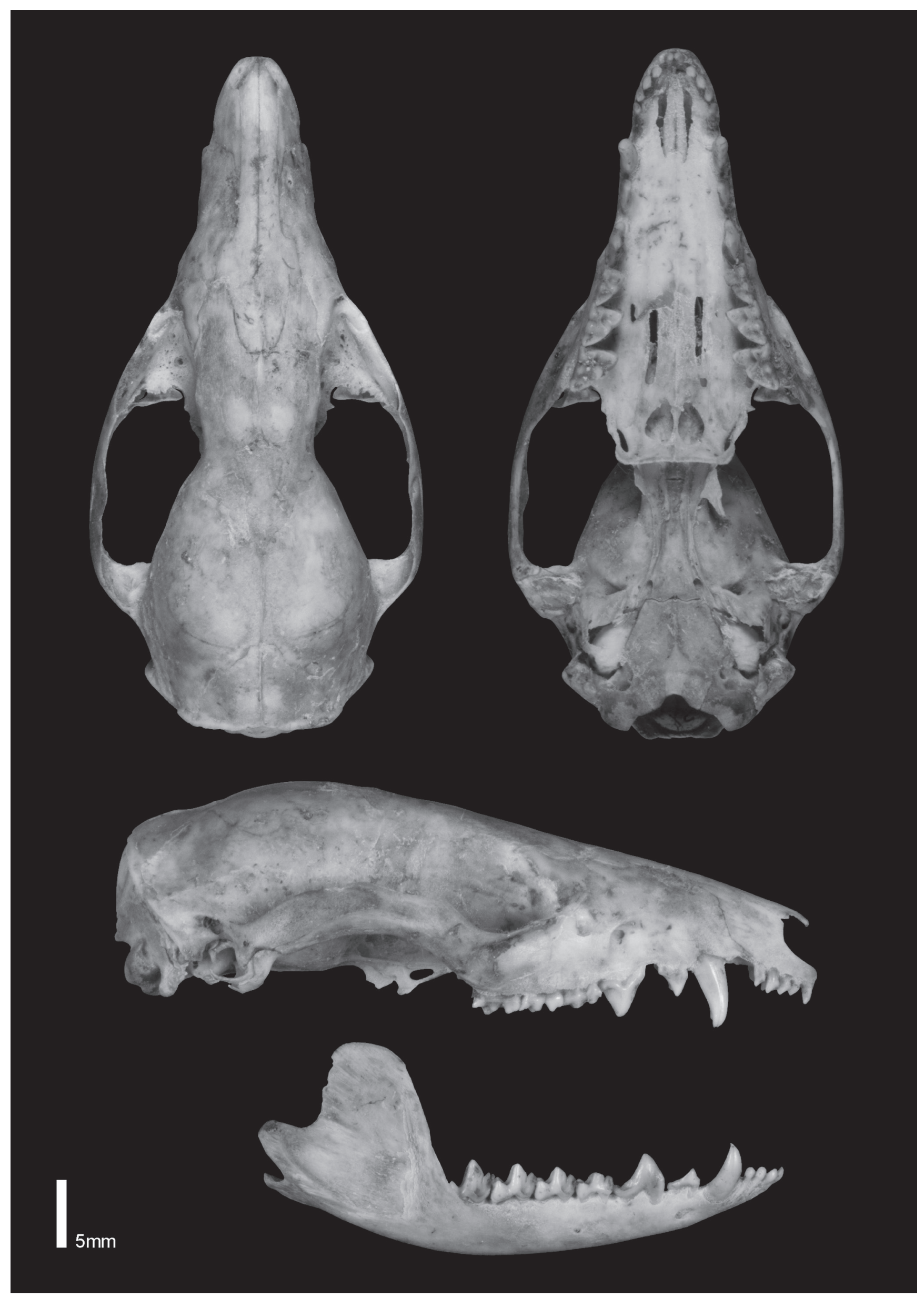

Figure 5. Skull and mandible of Philander quica (MZUSP 32516; male; $C B L=51.28 \mathrm{~mm}$ ). Skull on dorsal, ventral, and lateral view, and mandible on lateral view. 
by anteromedial strut of alisphenoid bulla, secondary foramen ovale present. Dorsal margin of foramen magnum bordered by supraoccipital and exoccipitals, incisura occipitalis present. Mandible with shallow notches (Fig. 5). Two mental foramina present on lateral surface of each hemimandible. Angular process acute and strongly inflected. C1 simple, without distinct accessory cusps. Upper molars without distinct ectoflexus.

Field observations: Only one $P$. quica specimen was collected at EEB, a juvenile male with P3 and M4 not erupted. It was captured with a Tomahawk trap in December, 2003.

\section{Tribe Marmosini Genus Monodelphis}

Monodelphis is the most speciose genus of SouthAmerican marsupials, with 23 currently recognized species (Pavan, 2019). In the AF about five species are known to occur (Pavan et al., 2014; Pavan, 2015). We identified 23 specimens of Monodelphis at EEB, which were assigned to two species: $M$. iheringi (17 specimens) and M. scalops (six specimens). The specimens from these two species can be reliably distinguished based on a set of external and cranio-dental characters. On the external morphology, $M$. iheringi exhibits three conspicuous black dorsal stripes in males and females throughout their lives - the stripes are relatively wider in adults and subadults and thinner in the juveniles, and the median stripe extends from the base of the tail to the snout, fading at the eye level. In $M$. scalops the dorsal stripes differ between males and females and are not conspicuous in all life stages - the dark stripes fade in both sexes, completely disappearing in adult males, while in females the stripes are still visible, although inconspicuously. Also, the dorsal pelage of $M$. scalops extends more conspicuously over the tail and reaches a greater proportion of it than in $M$. iheringi. Cranially, specimens of $M$. iheringi have the extracranial course of mandibular nerve completely exposed, while in M. scalops it is consistently enclosed by the posteromedial bullar lamina (secondary foramen ovale present). This characteristic was consistent in males and females and all age-groups of both species, and it is therefore an important diagnostic feature for the species (Fig. 6). The

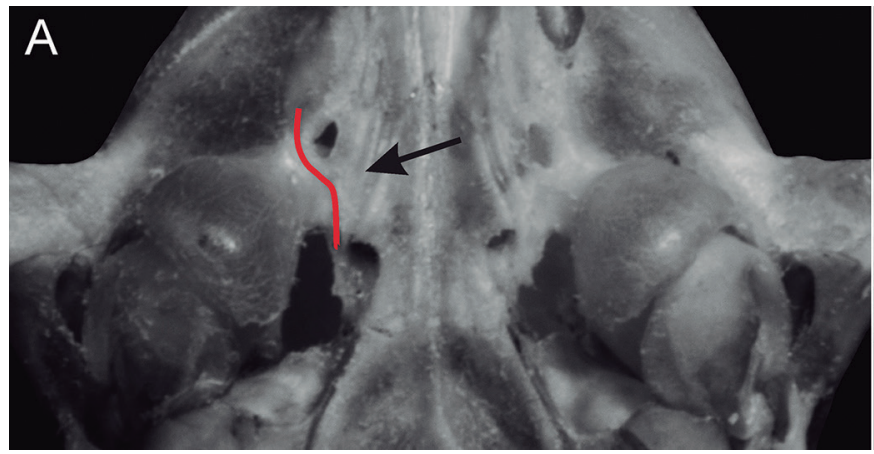

Table 3. Descriptive statistics (mean \pm standard deviation, range, and sample size) of body and cranio-dental measurements ( $\mathrm{mm}$ ), and weight ( $\mathrm{g}$ ), of Monodelphis iheringi and Monodelphis scalops from EEB. All measured specimens were adult males.

\begin{tabular}{lcc}
\hline & Monodelphis iheringi & Monodelphis scalops \\
\hline HBL & $98.23 \pm 4.60(94.9-105.0) 4$ & $(133.0) 1$ \\
TL & $53.90 \pm 4.15(51.0-60.0) 4$ & $(65.0) 1$ \\
HF1 & $15.80 \pm 1.09(14.2-16.5) 4$ & $(25.0) 1$ \\
HF2 & $17.08 \pm 1.21(15.3-18.0) 4$ & $(27.0) 1$ \\
EL & $11.20 \pm 1.29(9.3-12.0) 4$ & $(12.5) 1$ \\
W & $22.17 \pm 3.40(19.5-26.0) 3$ & $(74.0) 1$ \\
RW & $3.79 \pm 0.20(3.57-4.00) 4$ & $(3.72-6.31) 2$ \\
NB & $3.61 \pm 0.21(3.49-3.81) 4$ & $(3.88-5.39) 2$ \\
LIB & $4.87 \pm 0.06(4.80-4.94) 4$ & $(5.39-5.51) 2$ \\
ZB & $(12.37-20.35) 2$ \\
LBB & $13.16 \pm 0.71(12.48-14.00) 4$ & $(10.20-12.77) 2$ \\
NL & $9.86 \pm 0.30(9.41-10.04) 4$ & $(11.44-18.29) 2$ \\
RL & $11.15 \pm 0.47(10.54-11.61) 4$ & $(10.53-16.53) 2$ \\
CBL & $10.68 \pm 0.53(10.22-11.45) 4$ & $(25.42-35.30) 2$ \\
PL & $25.39 \pm 0.71(24.49-26.20) 4$ & $(14.14-19.42) 2$ \\
MAL & $13.49 \pm 0.53(12.80-14.07) 4$ & $(10.71-13.35) 2$ \\
M0L & $9.67 \pm 0.30(9.42-10.10) 4$ & $(5.78-5.83) 2$ \\
WM1 & $4.84 \pm 0.15(4.70-5.03) 4$ & $(1.23-1.35) 2$ \\
PW3 & $1.20 \pm 0.06(1.13-1.25) 4$ & $(7.33-9.68) 2$ \\
BBC & $7.32 \pm 0.24(7.01-7.56) 4$ & $(3.36-5.02) 2$ \\
B0L & $3.51 \pm 0.24(3.28-3.81) 4$ & $(3.05-5.26) 2$ \\
0CB & $3.43 \pm 0.04(3.38-3.47) 4$ & $(6.22-6.56) 2$ \\
\hline
\end{tabular}

smaller size of $M$. ihering in comparison with M. scalops is also evident in the cranio-dental morphometric characters (see Table 3 ).

\section{Monodelphis iheringi (Thomas 1888)}

Voucher material: $\mathrm{n}=17$; female: MZUSP 32519, 33783, 33784; uncatalogued: EEB 702; male: MZUSP 32520, 32521, 32522, 32523, 32524, 33785; uncatalogued: EBM 081, 169, 332; EEB 606, 757, 772; sex unknown: uncatalogued: EEB 528.

Specimens identification: Small size (see body measurements on Table 3). Dorsal pelage slightly dense and soft, brown lightly sprinkled with yellow ocher from head to near rump, and reddish brown on rump. Three stripes on dorsum, two lateral extending from the rump

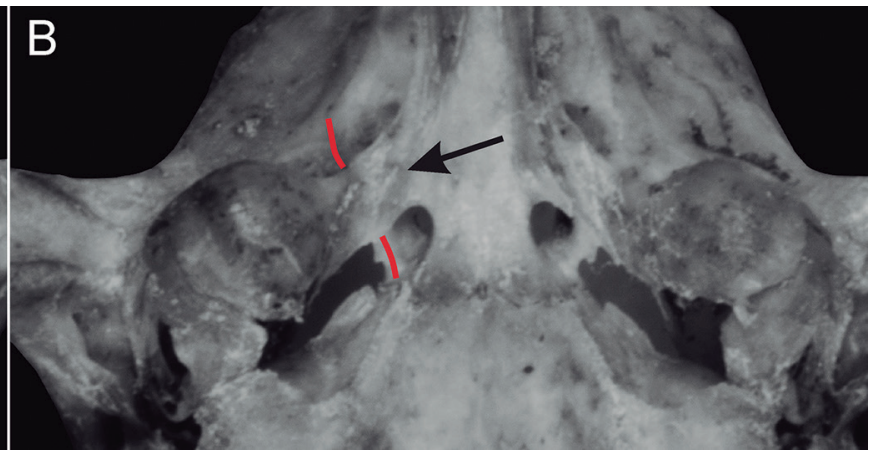

Figure 6. Conditions of extracranial course of mandibular nerve: (A) completely exposed in M. iheringi or (B) consistently enclosed by posteromedial bullar lamina in M. scalops. Schematic drawing of the mandibular nerve in red. 
to the shoulder girdle and a longer medial stripe, usually extending from the base of the tail to snout (in young specimens there is a variation in the length and intensity of the central stripe, which is less evident in the head, reaching or not the snout); dorsal stripes black with hairs entirely black. Lateral pelage brown or reddish-brown colored, sprinkled with yellow ocher; limit between lateral and ventral color strong. Ventral pelage orange colored on adult specimens and gray orange colored on young specimens; individual ventral hairs with basal half light gray and apical half orange. Scrotum with dark epithelium and covered by orange hairs. Mystacial vibrissae very short, not reaching the base of the pinnae when laid back. Pinnae small and rounded; covered with very short and sparse hairs, indistinguishable without magnification, from light brown to dark brown colored. Dorsal surface of manus covered by numerous hairs, dark brown and yellow ocher colored; digits with long claws (claw of dlll ranging from 2.2 to $2.9 \mathrm{~mm} ; \mathrm{n}=3$ ). Pes dorsally covered with numerous long hairs, dark brown and yellow ocher colored; dIII longer than dII and similar to IV; digits with long claws (claw of dIII ranging from 1.9 to $2.4 \mathrm{~mm} ; \mathrm{n}=3$ ); tufts of ungual hairs sparse and short, not extending beyond the claws, brownish colored. Tail shorter than head and body $(\approx 57 \%$ of head and body length, ranging from 48.6 to $67.2 \% ; n=15$ ); covered by short hairs, usually exceeding tree scales; scales very small, indistinguishable without magnification, with annular arrangement; tail dark brown dorsally with dark brown hairs and light brown ventrally with yellowish light brown hairs.

Skull short and broad (Fig. 7; Table 3). Premaxillary rostral process absent; premaxillae not extending beyond the alveolar margin of 11. Nasals long, extending anteriorly above 11, hiding nasal orifice from dorsal view; nasals conspicuously widened posteriorly than anteriorly. Interorbital region with margins generally parallels. Postorbital processes absent. Sagittal crest absent. Interparietal present. Two lacrimal foramina present on each side of skull, exposed on lateral view. Parietal and alisphenoid in contact on lateral braincase. Paroccipital process small and adnate to petrosal. Incisive foramina short, not surpassing C1. Premaxillary-maxillary suture collinear to superior canine alveolus, slightly moved anteriorly. Maxillopalatine fenestrae present; right and left fenestrae divided by a medial septum. Maxillary fenestrae absent. Palatine fenestrae absent. Posterolateral palatal foramina present and small, with complete ossified margins or margins incomplete. Transverse canal foramen present. Extracranial course of mandibular nerve opened. Dorsal margin of foramen magnum bordered by supraoccipital and exoccipitals, incisura occipitalis present. Mandible with deep notches (Fig. 7). Two mental foramina present on lateral surface of each hemimandible. Angular process acute and strongly inflected. Upper canine (C1) usually simple, without distinct accessory cusps or with vestigial posterior accessory cusps. First upper premolar (P1) large, with at least half of the height or width of the upper second premolar (P2). Third upper premolar (P3) distinctly taller than P2. Upper molars with distinct ectoflexus. Second lower premolar (p2) subequal in height to third lower premolar (p3).

Field observations: All 17 specimens of $M$. iheringi were collected using pitfall traps. Of those, 14 were captured in December, 2003, and three in April, 2004. Most were juveniles that did not display any sign of reproductive activity.

\section{Monodelphis scalops (Thomas 1888)}

Voucher material: $\mathrm{n}=6$; female: uncatalogued: EBM 179; male: uncatalogued: EBM 037, 092, 221; EEB 661, 836.

Specimens identification: Medium size (see body measurements on Table 3). Dorsal pelage on adult slightly dense and moderately soft, orange intense on the head, dark brown sprinkled with light yellow (grizzled appearance) between neck and middle dorsum, and reddish brown from middle dorsum to rump; dorsal stripes absent. Dorsal pelage on young specimens dense and soft, reddish brown on the head, dark brown slightly sprinkled with light yellow from neck to rump, and reddish brown on rump; three smooth and relatively broad sagittal dorsal stripes, extending from shoulder girdle to rump, dark brown colored with hairs entirely dark brown. Lateral pelage color very similar to dorsal color in both adult and young specimens; limit between lateral and ventral color strong on young and very weak on adult specimens. Ventral pelage yellowish gray colored (grizzled appearance) on adult and mostly grayish on young; individual ventral hairs with basal band light gray $(1 / 3)$, central band dark gray (1/3) and apical band yellow (1/3) on adult and with basal band light gray $(1 / 2)$ and apical band yellow $(1 / 2)$ on young specimens. Scrotum with dark epithelium and covered by orange hairs. Mystacial vibrissae very short, not reaching the base of the pinnae when laid back. Pinnae small and rounded; covered with very short and sparse hairs, indistinguishable without magnification, generally dark brown. Dorsal surface of manus covered with numerous and long hairs, dark brown colored; digits with long claws (claw of dIII measuring $3.45 \mathrm{~mm}$ on one adult specimen and $2.25 \mathrm{~mm}$ on one young specimen). Pes dorsally covered by numerous and long hairs, generally light brown colored, reddish on adult specimens; dIII longer than dII and similar to IV; digits with long claws (claw of dlll measuring $3.35 \mathrm{~mm}$ on one adult specimen and $2.25 \mathrm{~mm}$ on one young specimen); tufts of ungual hairs sparse and short, not extending beyond the claws, dark brown colored. Tail shorter than head and body ( $\approx 48 \%$ of head and body length, ranging from 44.9 to $51.5 \% ; n=5$ ); covered at base (approximately $1 / 6$ ) with pelage similar to dorsum and after that covered by short hairs, usually tree scales longer; scales very small, indistinguishable without magnification, with annular arrangement; tail dorsal color brown with brown slight reddish hairs and ventral color brown, slightly paler, with yellowish light brown hairs. 

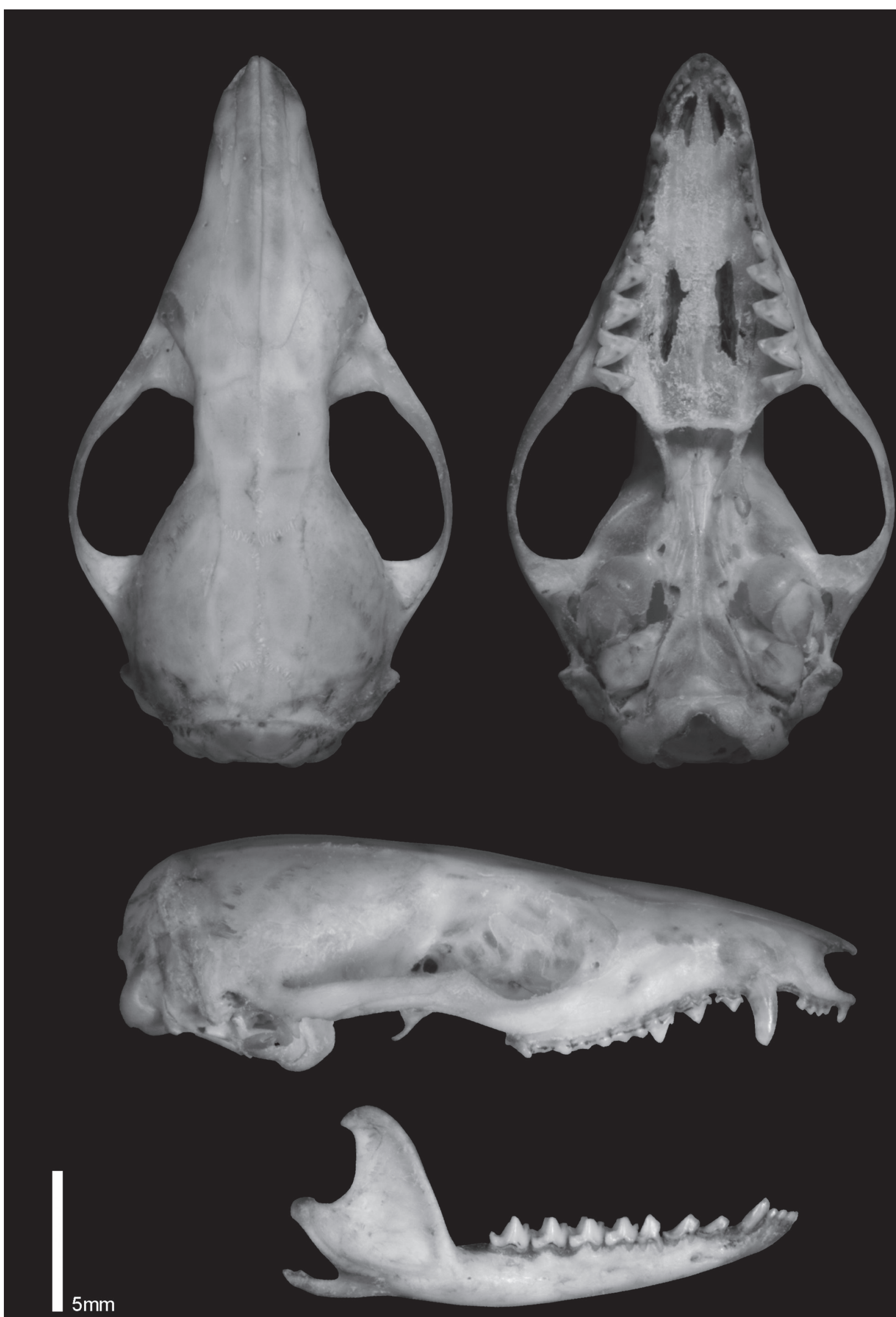

Figure 7. Skull and mandible of Monodelphis iheringi (EEB 772; male; $(B L=25.58 \mathrm{~mm})$. Skull on dorsal, ventral, and lateral view, and mandible on lateral view. 
Skull short and broad (Fig. 8; Table 3). Premaxillary rostral process absent; premaxillae not extending beyond the alveolar margin of I1. Nasals long, extending anteriorly above 11 , hiding nasal orifice from dorsal view; nasals conspicuously widened posteriorly than anteriorly. Interorbital region with margins parallels or slight posteriorly convergent. Postorbital processes absent. Sagittal crest present and extending over frontals. Interparietal present. Two lacrimal foramina present on each side of skull, exposed on lateral view. Parietal and alisphenoid in contact on lateral braincase. Paroccipital process small and adnate to petrosal. Incisive foramina short, not surpassing C1. Premaxillary-maxillary suture collinear to the alveolus of upper canine, slightly moved anteriorly. Maxillopalatine fenestrae present; right and left fenestrae divided by a medial septum. Maxillary fenestrae absent. Palatine fenestrae absent. Posterolateral palatal foramina present and large, with complete ossified margins. Transverse canal foramen present. Extracranial course of mandibular nerve enclosed by anteromedial strut of alisphenoid bulla, secondary foramen ovale present. Dorsal margin of foramen magnum bordered by supraoccipital and exoccipitals, incisura occipitalis present. Mandible with deep notches (Fig. 8). Two mental foramina present on lateral surface of each hemimandible. Angular process acute and strongly inflected. C1 simple, without distinct accessory cusps. P1 large, with at least half of the height or width of the P2. P3 slightly larger than P2. Upper molars with distinct ectoflexus. p2 larger than $\mathrm{p} 3$.

Field observations: Six M. scalops were collected at EEB: one specimen in December, 2003, four in April, 2004, and one in May, 2004. Five of those were captured with pitfall traps and one with conventional trap.

\section{Tribe Thylamyini \\ Gracilinanus microtarsus (Wagner 1842)}

Voucher material: $\mathrm{n}=4$; female: uncatalogued: EBM 043; EEB 822; male: uncatalogued: EEB 859; sex unknown: uncatalogued: EEB 857.

Specimens identification: Small size (see body measurements on Table 4). Head pelage brown, distinctly lighter in the mid-rostral region from snout to eyes; presence of a black mask around the eyes, very close to the base of the ears. Dorsal pelage very dense and soft, homogeneous colored from brown to light brown. Lateral pelage light brown; limit between lateral and ventral color marked. Ventral pelage entirely grayish cream; individual hairs with basal portion dark gray (approximately $2 / 3$ ) and apical portion cream (1/3). Mystacial vibrissae long, reaching the base of pinnae when laid back but not surpassing the top of them. Pinnae large and slight oval in shape, orange yellow colored at the base and brown on the apical portion; covered with short and very sparse hairs, whitish colored at the base and brown on the apical portion. Dorsal surface of manus covered by short hairs, whitish colored; digits with short claws, claw of dIII $=1.5 \mathrm{~mm}$. Pes dorsally covered by short hairs, whitish colored; dIII similar in length to dII and IV; digits with short claws, claw of dIII $=2.15 \mathrm{~mm}$; tufts of ungual hairs sparse and short, not extending beyond the claws, whitish colored. Tail longer than head and body (rang-

Table 4. Body and cranio-dental morphometric values $(\mathrm{mm})$, and weight $(\mathrm{g})$ of Gracilinanus microtarsus from EEB, and descriptive statistics (mean \pm standard deviation, range, and sample size) of "small microtarsus group" (Lóss et al., 2011).

\begin{tabular}{|c|c|c|c|c|c|}
\hline & \multicolumn{2}{|r|}{ Males } & \multicolumn{3}{|c|}{ Females } \\
\hline & EEB 859 & "small microtarsus group" & EEB 822 & EBM 043 & "small microtarsus group" \\
\hline HBL & - & $100.06 \pm 11.94(72.0-127.0) 58$ & - & 84.6 & $98.80 \pm 20.14(67.0-185.0) 30$ \\
\hline TL & - & $144.80 \pm 16.33(70.0-175.0) 59$ & - & 131.0 & $143.33 \pm 13.55(110.0-171.0) 30$ \\
\hline HF1 & - & $17.10 \pm 1.65(13.0-20.0) 60$ & - & 16.2 & $16.95 \pm 2.53(13.0-27.0) 29$ \\
\hline EL & - & $19.78 \pm 1.91(14.0-22.0) 54$ & - & 15.0 & $18.94 \pm 2.31(13.0-22.0) 28$ \\
\hline w & - & $23.71 \pm 7.16(12.0-50.0) 52$ & - & 14.5 & $21.61 \pm 9.01(12.0-58.0) 27$ \\
\hline RW & 4.16 & - & 4.98 & - & - \\
\hline NB & 3.49 & $3.32 \pm 0.30(2.65-4.04) 65$ & 4.28 & - & $3.30 \pm 0.27(2.72-3.98) 35$ \\
\hline LIB & 5.04 & $4.96 \pm 0.22(4.40-5.37) 65$ & 5.51 & - & $4.88 \pm 0.23(4.39-5.42) 35$ \\
\hline ZB & 15.52 & $15.77 \pm 0.84(14.45-17.98) 63$ & 17.35 & - & $15.60 \pm 0.95(14.0-17.46) 33$ \\
\hline BB & 11.64 & $11.56 \pm 0.35(10.62-12.39) 64$ & 12.77 & - & $11.37 \pm 0.42(9.94-11.96) 35$ \\
\hline NL & 12.99 & $11.79 \pm 0.81(10.05-13.97) 61$ & 13.01 & - & $11.47 \pm 0.90(9.93-13.42) 31$ \\
\hline RL & 11.20 & - & 11.52 & - & - \\
\hline CBL & 28.73 & $29.09 \pm 1.14(26.26-31.29) 65$ & 29.93 & - & $28.43 \pm 1.31(25.8-30.70) 33$ \\
\hline PL & 16.01 & $15.66 \pm 0.74(13.48-17.10) 62$ & 16.55 & - & $15.28 \pm 0.75(13.78-16.89) 34$ \\
\hline MAL & 11.13 & $11.10 \pm 0.38(10.05-11.89) 65$ & 11.39 & - & $11.04 \pm 0.32(10.47-11.91) 35$ \\
\hline MOL & 5.69 & $5.68 \pm 0.18(5.24-6.10) 65$ & 5.83 & - & $5.72 \pm 0.17(5.41-6.11) 35$ \\
\hline WM1 & 1.35 & - & 1.49 & - & - \\
\hline PW4 & 8.32 & $8.78 \pm 0.32(8.11-9.59) 64$ & 9.39 & - & $8.88 \pm 0.38(8.31-9.77) 35$ \\
\hline BBC & 5.19 & - & 5.77 & - & - \\
\hline BOL & 3.29 & - & 3.92 & - & - \\
\hline$O C B$ & 6.46 & - & 6.58 & - & - \\
\hline
\end{tabular}



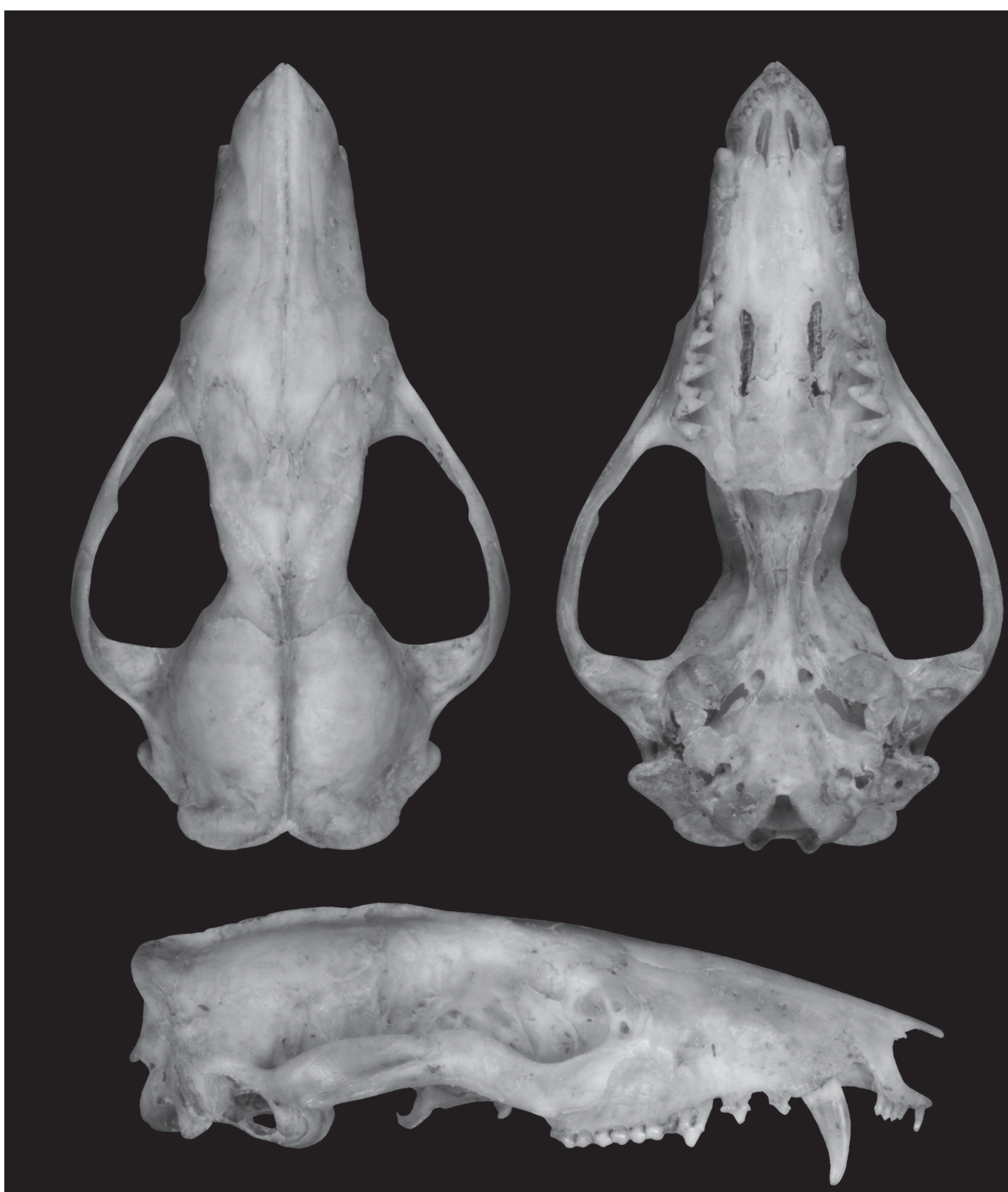

$5 \mathrm{~mm}$

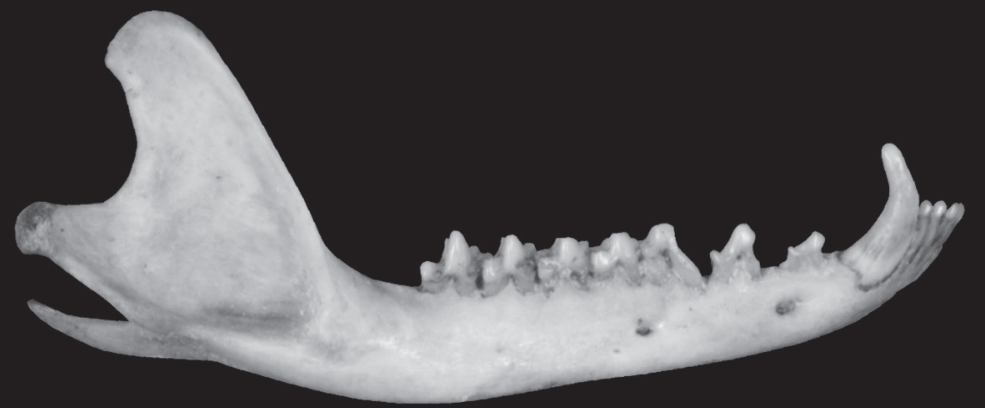

Figure 8. Skull and mandible of Monodelphis scalops (EEB 661; male; $(B L=35.30 \mathrm{~mm})$. Skull on dorsal, ventral, and lateral view, and mandible on lateral view. 
ing from 155 to $157 \%$ of head and body length; $n=2$ ); covered by short hairs, three hairs usually with same size (from one to two scales longer) emerging from distal margin of each scale; scales small, indistinguishable to the naked eye, with spiral arrangement; tail dorsal color brown, with hairs mostly brown, and ventral color brown slightly lighter, with hair predominantly whitish.

Skull short and broad (Fig. 9; Table 4). Premaxillary rostral process present; premaxillae extending beyond the alveolar margin of I1. Nasals long, extending anteriorly above I1, hiding nasal orifice from dorsal view; nasals conspicuously widened posteriorly than anteriorly; posterior margins of nasals convergent. Interorbital region with margins mostly parallels and rounded. Postorbital processes absent. Sagittal crest absent. Interparietal present. Two lacrimal foramina present on each side of skull, but not exposed on lateral view. Parietal and alisphenoid in contact on lateral braincase. Paroccipital process small and adnate to petrosal. Incisive foramina reaching the posterior face of $\mathrm{C} 1$ or slightly surpassing. Premaxillarymaxillary suture collinear to superior canine alveolus. Maxillopalatine fenestrae present and large; right and left fenestrae divided by a medial septum. Maxillary fenestrae present. Palatine fenestrae present and large. Posterolateral palatal foramina present, with complete ossified margins. Transverse canal foramen present. Extracranial course of mandibular nerve consistently enclosed by anteromedial strut of alisphenoid bulla, secondary foramen ovale present. Dorsal margin of foramen magnum bordered by supraoccipital and exoccipitals, incisura occipitalis present. Mandible with deep notches (Fig. 9). Two mental foramina present on lateral surface of each hemimandible. Angular process acute and strongly inflected. C1 simple, without distinct accessory cusps. P1 large, with at least half of the height or width of the P2. P2 slightly larger them P3. Upper molars with distinct ectoflexus in one or more teeth. p2 slightly larger than p3.

Remarks: Lóss et al. (2011) examined the geographical variation in Gracilinanus microtarsus using different sources of information (e.g., genetic, morphometric, morphologic traits). They recovered this taxon as monotypic but with strong geographical structuration. Based on morphology (both quali and quantitative characters) three distinct groups were suggested by Lóss et al. (2011). Based on our examinations - and also in accordance with the geographical distribution - the phenotype of EBB specimens fits into the "small microtarsus" group, which is distinguished by ventral hairs with gray base from the chin to the base of the tail, and bicolored tail. In the skull, specimens of this group have poorly developed temporal margins and absent or poorly developed supraorbital crests; incisive foramen short, usually not extended beyond the canines; and tympanic process of alisphenoid inflated. Biometric comparisons between EEB specimens with those in the "small microtarsus" group (Lóss et al., 2011) are presented in Table 4.

Field observations: Four specimens of G. microtarsus were collected at EEB, all with pitfall traps in 2004. Two specimens were collected in April and two in September. Information on the reproductive condition of these specimens were not available.

\section{Marmosops paulensis (Tate 1931)}

Voucher material: $\mathrm{n}=9$; female: MZUSP 32512, 32513, 32514, 32515; uncatalogued: EBM 038; EEB 1021; male: uncatalogued: EEB 860, 1015; sex unknown: uncatalogued: EBM 308.

Specimens identification: Medium size (see body measurements on Table 5). Head pelage predominantly brown; dark brown mid-rostral stripe present, but narrow; dark brown circumocular mask present. Dorsal pelage soft and moderately dense, homogeneously brown colored, slightly darker on the mid-dorsal region from shoulder girdle to rump. Lateral pelage brown colored, very similar to dorsum; limit between lateral and ventral color strong; presence of a subtle stripe with hairs cream colored at base and brown at top on the lateral-ventral transition. Ventral pelage entirely cream white; individual hairs with basal and apical portions cream white; hairs on the mammary region yellow-ocher colored in females on reproductive activity; scrotum with brown epithelium and covered by cream/white hairs. Mystacial vibrissae long, reaching the base of pinnae when laid back but not surpassing the top of them. Pinnae large and rounded, yellowish colored at the base and brown on the apical portion; covered with very short and sparse hairs, whitish colored at the base and brown on the apical portion. Dorsal surface of manus covered by numerous hairs, predominantly brown colored on the metacarpal region and whitish over digits; digits with short claws, claw of dIII ranging from $1.5 \mathrm{~mm}$ to $2.0 \mathrm{~mm}(\mathrm{n}=6)$. Pes dorsally covered by numerous hairs, mostly whitish colored; dIII similar in length to dII and IV; digits with medium claws, claw of dlll ranging from $2.8 \mathrm{~mm}$ to $3.3 \mathrm{~mm}$ $(n=6)$; tufts of ungual hairs sparse and very short, not extending beyond the claws, whitish colored. Tail longer than head and body ( $\approx 133 \%$ of head and body length, ranging from 104.5 to $148.3 \% ; n=8$ ); covered by short hairs, three hairs emerging from distal margin of each scale, central hair longer (length of two scales) and wider them lateral hairs; scales medium in size, distinguishable to the naked eye, with spiral arrangement; tail usually particolored, basal portion brown colored in the dorsum, with brown hairs, and brown slightly lighter in the venter, with whitish hairs, and apical portion of the tail (ranging from $1 / 3$ to $1 / 4$ ) unpigmented on both surfaces, with whitish hairs.

Skull slender and elongated (Fig. 10; Table 5). Premaxillary rostral process present but very short; premaxillae extending subtly beyond the alveolar margin of I1. Nasals long, extending anteriorly above 11, hiding nasal orifice from dorsal view; nasals uniformly narrow with lateral margins parallels, widened only on the maxillary-frontal suture region; posterior margins of nasals divergent. Interorbital region slightly anteriorly diver- 


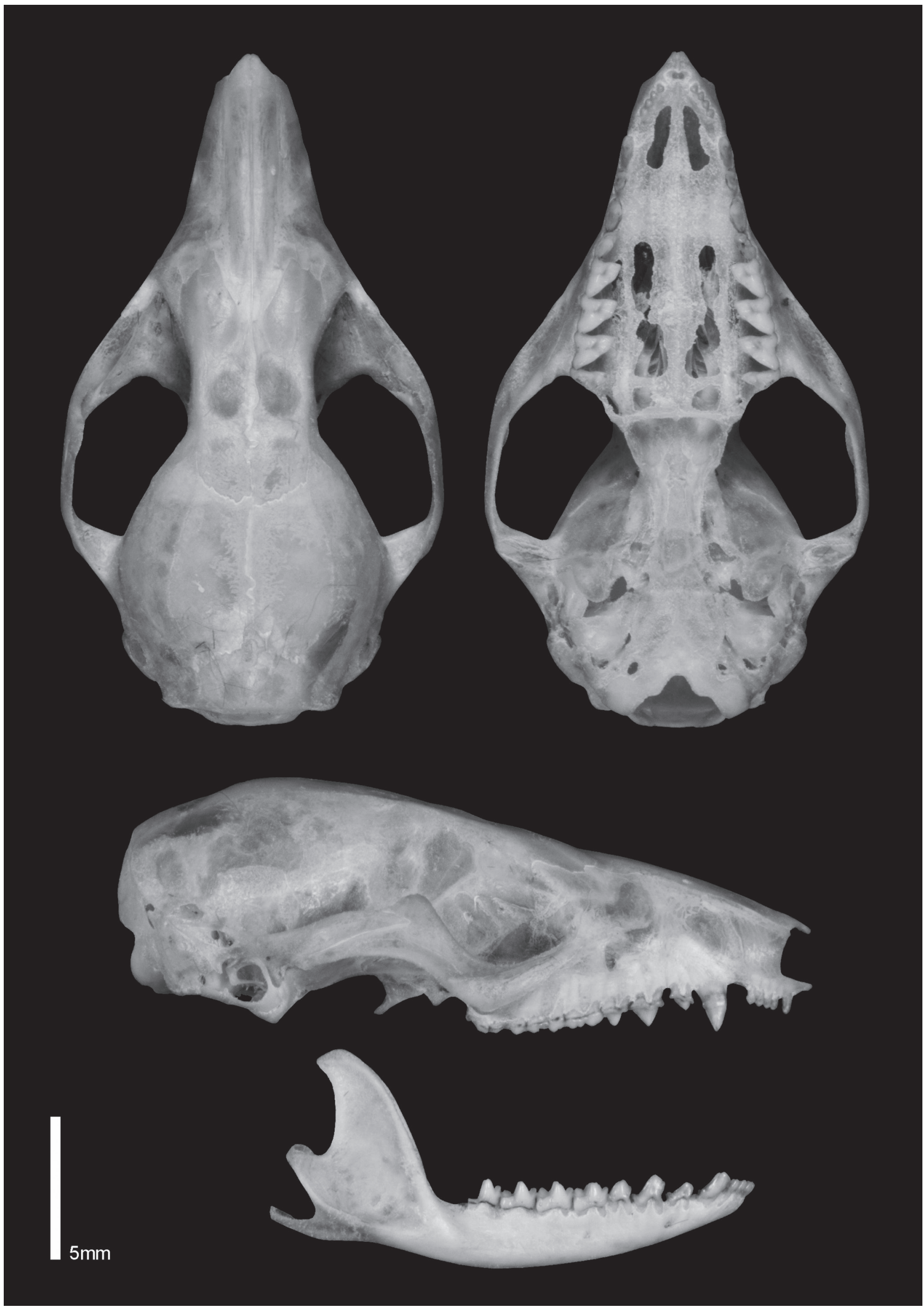

Figure 9. Skull and mandible of Gracilinanus microtarsus (EEB 822; female; $(B L=29.93 \mathrm{~mm})$. Skull on dorsal, ventral, and lateral view, and mandible on lateral view. 
Table 5. Descriptive statistics (mean \pm standard deviation, range, and sample size) of body and cranio-dental measurements ( $\mathrm{mm}$ ), and weight ( $\mathrm{g}$ ), of adult specimens of Marmosops paulensis from EEB, and descriptive statistics of M. paulensis and M. incanus (Mustrangi \& Patton, 1997).

\begin{tabular}{|c|c|c|c|c|c|c|}
\hline \multirow[b]{4}{*}{ TTL } & \multicolumn{4}{|c|}{ M. paulensis } & \multicolumn{2}{|c|}{ M. incanus } \\
\hline & \multicolumn{2}{|c|}{ Males } & \multicolumn{2}{|c|}{ Females } & Males & Females \\
\hline & EEB specimens & Species values & EEB specimens & Species values & \multicolumn{2}{|c|}{ Species values } \\
\hline & 307.0 & $320.8 \pm 4.6(264-365) 26$ & $319.6 \pm 20.72(296-350) 5$ & $281.1 \pm 5.8(243-320) 19$ & $343.0 \pm 2.85(273-431) 138$ & $304.1 \pm 3.1(240-358) 93$ \\
\hline TL & 179.0 & $189.7 \pm 2.86(150-212) 26$ & $183.0 \pm 7.58(171-190) 5$ & $164.0 \pm 2.22(145-181) 19$ & $196.7 \pm 1.60(146-237) 138$ & $175.6 \pm 1.7(140-200) 93$ \\
\hline HF2 & 23.5 & $21.9 \pm 0.26(19-24) 26$ & $21.60 \pm 0.42(21-22) 5$ & $19.6 \pm 0.33(17-22) 22$ & $22.1 \pm 0.18(15-28) 139$ & $19.5 \pm 0.24(11-24) 93$ \\
\hline EL & 22.5 & $23.5 \pm 0.29(20-27) 26$ & $23.10 \pm 1.64(21.5-25.5) 5$ & $21.3 \pm 0.26(19-23) 22$ & $27.2 \pm 0.22(20-33) 137$ & $25.5 \pm 0.21(21-32) 93$ \\
\hline W & 67.0 & $42.7 \pm 2.51(20-70) 25$ & $60.40 \pm 4.98(53-65) 5$ & $28.0 \pm 2.26(16-47) 19$ & $66.5 \pm 2.11(25-140) 127$ & $42.5 \pm 1.57(20-72) 82$ \\
\hline RW & 5.65 & $5.98 \pm 0.07(5.4-6.7) 25$ & $5.71 \pm 0.30(5.4-6.11) 5$ & $5.65 \pm 0.10(5.1-6.4) 21$ & $6.75 \pm 0.05(5.8-8.2) 131$ & $6.16 \pm 0.05(5.4-7.3) 84$ \\
\hline NB & 4.16 & - & $3.93 \pm 0.28(3.71-4.41) 5$ & - & - & - \\
\hline LIB & 6.86 & $6.52 \pm 0.06(5.9-7.0) 26$ & $6.54 \pm 0.14(6.3-6.67) 5$ & $6.13 \pm 0.06(5.6-6.8) 21$ & $6.73 \pm 0.04(5.6-8.5) 133$ & $6.24 \pm 0.04(5.5-7.4) 86$ \\
\hline ZB & 19.08 & $17.40 \pm 0.30(12.8-20.1) 24$ & $18.81 \pm 0.46(18.12-19.2) 5$ & $16.51 \pm 0.24(15.1-18.7) 20$ & $19.87 \pm 0.14(17.1-23.7) 126$ & $17.94 \pm 0.14(16.0-20.4) 76$ \\
\hline BB & 13.48 & - & $13.66 \pm 0.30(13.36-14.01) 5$ & - & - & - \\
\hline NL & 17.39 & $17.18 \pm 0.20(15.0-19.0) 24$ & $18.72 \pm 0.64(18.1-19.58) 5$ & $15.81 \pm 0.25(14.2-18.1) 21$ & $19.38 \pm 0.14(14.9-22.8) 131$ & $17.2 \pm 0.15(14.6-21.0) 85$ \\
\hline $\mathbf{R L}$ & 15.4 & - & $16.23 \pm 0.53(15.6-16.94) 5$ & - & - & - \\
\hline CBL & 36.03 & $36.67 \pm 0.35(32.6-39.9) 24$ & $36.92 \pm 0.92(36.0-38.4) 5$ & $34.43 \pm 0.44(31.4-37.8) 20$ & $40.08 \pm 0.24(33.0-45.0) 122$ & $36.37 \pm 0.25(32.1-41.2) 76$ \\
\hline PL & 20.72 & - & $21.12 \pm 0.35(20.79-21.65) 5$ & - & - & - \\
\hline MAL & 14.95 & - & $14.95 \pm 0.41(14.39-15.35) 5$ & - & - & - \\
\hline MOL & 7.21 & $7.33 \pm 0.04(6.7-7.7) 26$ & $7.11 \pm 0.15(6.95-7.32) 5$ & $7.13 \pm 0.03(6.9-7.4) 21$ & $7.43 \pm 0.02(6.5-8.1) 132$ & $7.21 \pm 0.03(6.4-7.7) 88$ \\
\hline WM1 & 1.49 & - & $1.53 \pm 0.03(1.49-1.55) 5$ & - & - & - \\
\hline PW3 & 10.63 & $10.00 \pm 0.07(9.4-10.7) 25$ & $10.72 \pm 0.19(10.45-10.95) 5$ & $9.59 \pm 0.08(9.0-10.3) 21$ & $10.58 \pm 0.05(9.3-11.6) 131$ & $10.02 \pm 0.05(8.3-11.3) 83$ \\
\hline $\mathrm{BBC}$ & 5.79 & - & $6.09 \pm 0.27(5.71-6.43) 5$ & - & - & - \\
\hline BOL & 4.64 & - & $4.35 \pm 0.07(4.22-4.41) 5$ & - & - & - \\
\hline$O C B$ & 7.7 & - & $7.89 \pm 0.17(7.76-8.18) 5$ & - & - & - \\
\hline HC1 & 3.37 & $2.33 \pm 0.10(1.51-3.58) 26$ & $2.35 \pm 0.11(2.19-2.48) 5$ & $1.83 \pm 0.06(1.54-2.37) 21$ & $3.12 \pm 0.05(1.75-4.47) 132$ & $2.84 \pm 0.03(1.76-2.84) 88$ \\
\hline
\end{tabular}

gent, with margins predominantly parallels and rounded, without crests. Postorbital processes absent. Sagittal crest absent. Interparietal present. Two lacrimal foramina present on each side of skull, but not exposed on lateral view. Parietal and alisphenoid in contact on lateral braincase. Paroccipital process small and fused to petrosal. Incisive foramina surpassing $\mathrm{C} 1$ and reaching P1. Premaxillary-maxillary suture collinear to superior canine alveolus. Maxillopalatine fenestrae present; right and left fenestrae divided by a medial septum. Maxillary fenestrae usually present and reduced. Palatine fenestrae with large variation (Fig. 11): absent (one specimen), present only on the left side (one specimen), present on both sides but small (one specimen), present and large (three specimens). Posterolateral palatal foramina present, with complete ossified margins. Transverse canal foramen present (three specimen) or absent (three specimens). Extracranial course of mandibular nerve consistently enclosed by anteromedial strut of alisphenoid bulla, secondary foramen ovale present. Dorsal margin of foramen magnum bordered by supraoccipital and exoccipitals, incisura occipitalis present. Mandible with deep notches (Fig. 10). Two mental foramina present on lateral surface of each hemimandible. Angular process acute and strongly inflected. C1 without distinct accessory cusps (four specimens), with posterior accessory cusps (one specimen), or with posterior and anterior accessory cusps (one specimen). P1 large, with at least half of the height or width of the P2. P2 and P3 subequal in height. Upper molars with distinct ectoflexus only on M3. p2 slightly taller than p3 (four specimens) or p2 and p3 subequal in height (two specimens).
Remarks: Mustrangi \& Patton (1997) in a taxonomic review of the AF forms of Marmosops recognized $M$. paulensis (Marmosa incana paulensis Tate) and M. incanus (including Marmosa incana bahiensis Tate and Marmosa incana incana Tate) as two distinct and valid species. These two species were separated based on their divergence in the mitochondrial gene CYTB (about 17\%) and on external and cranial characters. In general, our analysis of EEB specimens corroborated their suggestions, reinforcing the relevance of some traits to distinguish between M. paulensis and M. incanus. Among them, the following characters of $M$. paulensis stand out: dorsal coloration brown more intensely reddish; well defined contrast in the transition between the lateral and ventral pelage, without the presence of a band of hairs with gray base laterally on the venter; posterior margins of nasal divergent; and long incisive foramen, which greatly exceeds the level of upper canines anteriorly. However, we have observed important variation in the palatine fenestrae: while Mustrangi \& Patton (1997: 42) mentioned that these fenestrae are usually absent or greatly reduced in $M$. paulensis, specimens from the EEB showed large variation in this character, with some specimens also presenting well developed palatine fenestrae (see Fig. 11).

A comparison between the morphometric values obtained from the EEB specimens with those published by Mustrangi \& Patton (1997) reveals that the specimens from the EEB specimens are larger on average, with higher maximum values. This applies to most body and some cranial variables. However, the important characters that distinguish $M$. paulensis from $M$. incanus (e.g., length 

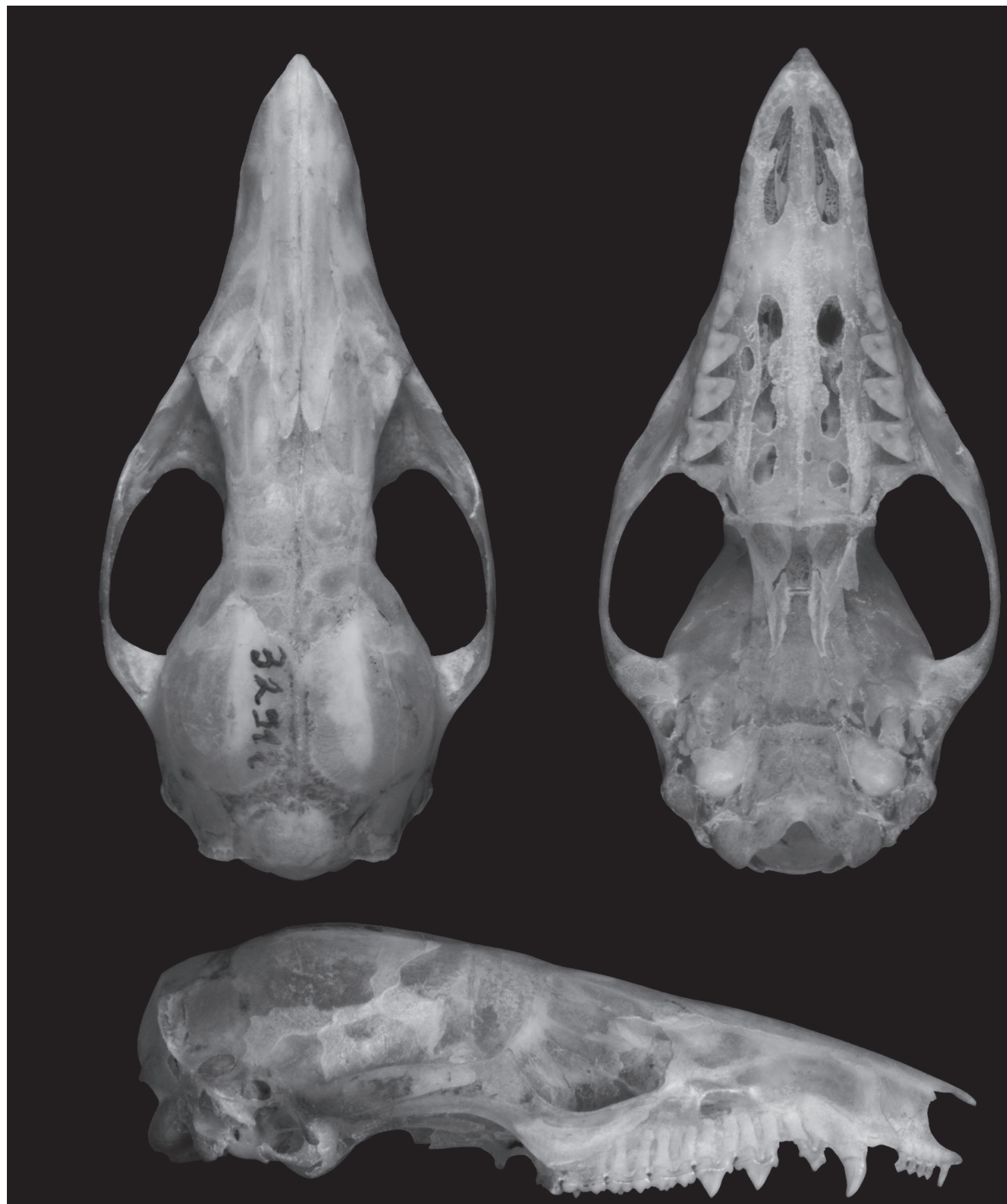

$5 \mathrm{~mm}$

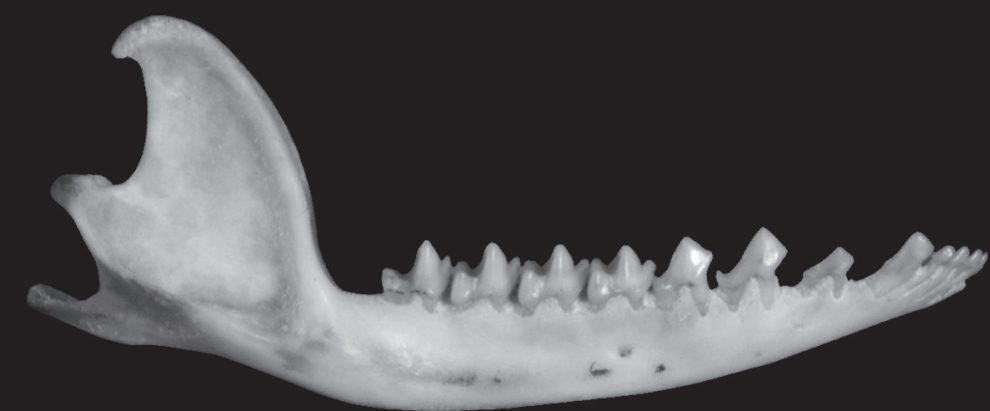

Figure 10. Skull and mandible of Marmosops paulensis (MZUSP 32512; female; $C B L=36.44 \mathrm{~mm}$ ). Skull on dorsal, ventral, and lateral view, and mandible on lateral view. 
of ear and height of upper canine), though presenting higher means in the EEB specimens, fall into the variation proposed for M. paulensis (Table 5).
Field observations: Nine M. paulensis were collected at EEB, six in pitfall traps and three with conventional traps. The specimens were collected in December 2003 (four

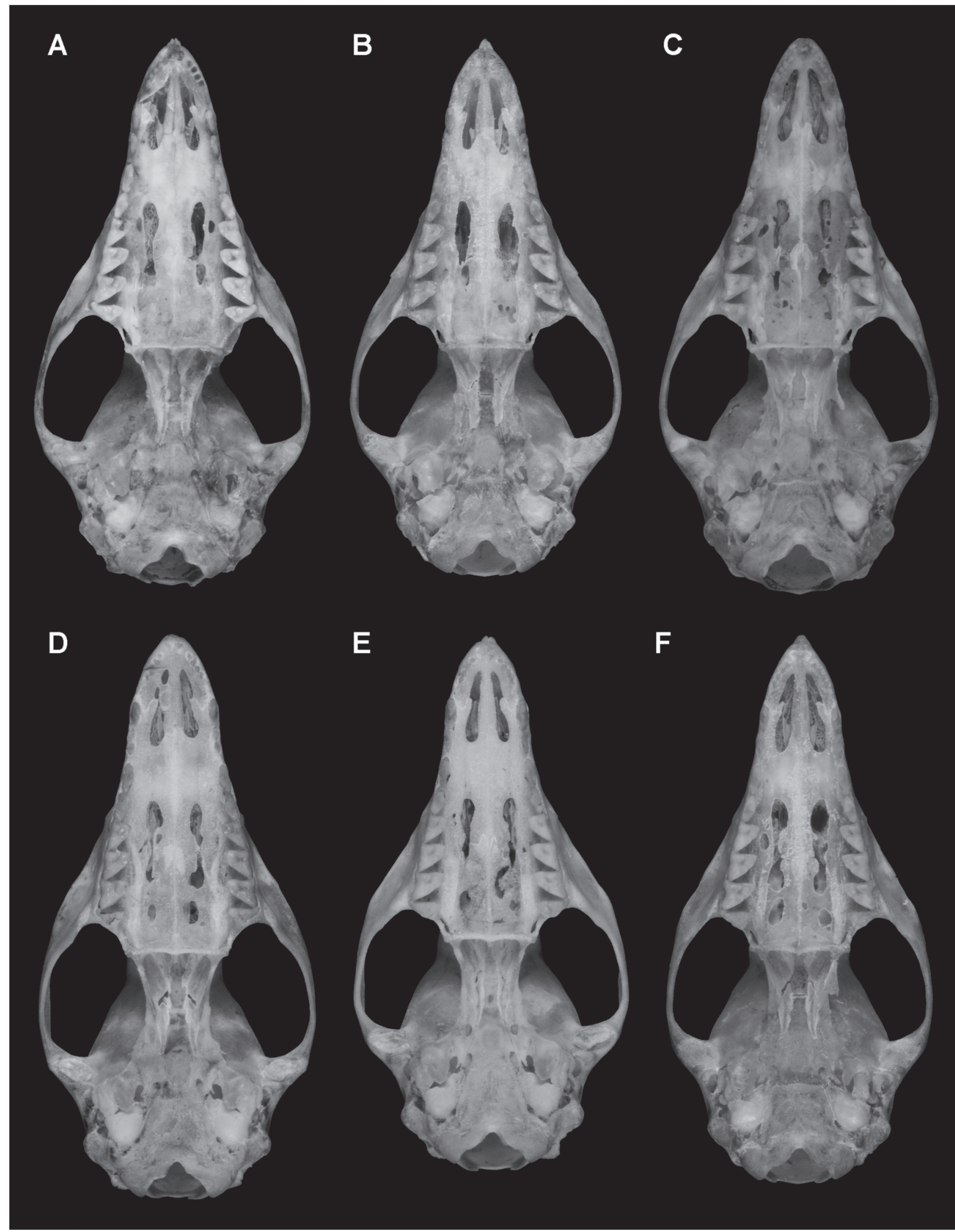

Figure 11. Variation of palatine fenestrae in Marmosops paulensis: absent in MZUSP 32514 (A); present only on the left side in MZUSP 32515 (B); present on both sides but small in MZUSP 32513 (C); and present and large in EEB 1021 (D), EEB 1015 (E), and MZUSP 32512 (F). 
specimens), April 2004 (two specimens), September 2004 (one specimens) and December 2011 (two specimens). In December 2003, three reproductively active females (mammal glands visible and lactating) were collected along with one female that showed no signs of reproductive activity. In April 2004 one reproductively active female and one female that showed no signs of reproductive activity were collected. In September 2004 one adult male was collected. In December 2011, an adult male and a lactating female were collected.

\section{Order Rodentia \\ Family Cricetidae Subfamily Sigmodontinae}

\section{Body-size categories of sigmodontine rodents}

The values of $\mathrm{HBL}$ and $\mathrm{W}$ enabled us to establish four body-size classes for sigmodontine rodents. On the biplot, two very clear discontinuities in the dispersion of the values resulted in the recognition of three classes (Fig. 12), as follows: (1) small - length of head and body shorter than $100 \mathrm{~mm}$ and weighing up to $30 \mathrm{~g}$; (2) medium - length of head and body between 101 and $130 \mathrm{~mm}$ and weight between 31 and $60 \mathrm{~g}$; and (3) large - length of head and body between 131 and $160 \mathrm{~mm}$ and weight ranging from 61 to $100 \mathrm{~g}$. Moreover, we consider a fourth class (very large) for species in with HBL and W values extrapolate those in class 3 (e.g., Nectomys squamipes).

\section{Tribe Akodontini Genus Akodon}

Akodon is the most speciose genus of the tribe Akodontini (Pardiñas et al., 2015). In the AF, six species have been recorded, three of which potentially occur at EEB - Akodon cursor, A. montensis and A. paranaensis (Paglia et al., 2012; Pardiñas et al., 2015). These species are morphologically very similar and their identification relies mostly on karyotype and/or molecular data (Christoff et al., 2000; Geise et al., 2005; Gonçalves et al., 2007). Therefore, to identify the large series of specimens from EEB we used three sources of information: karyotypes (provided by Geise et al., 2005 for nine specimens), CYTB sequences (generated for 14 specimens; data not shown), and cranio-dental morphometrics (using all specimens).

The PCA analysis, based on the values of 19 cranio-dental variables, showed two clusters in the Akodon sample. The projections of the individual scores of the specimens are shown in Fig. 13. The first component accounted for $93.49 \%$ of the variation and no discontinuity in the projection of individual scores was observed; in the second component, which corresponded to $1.46 \%$ of the variation, there was a clear separation between one specimen and all the others; the third component accounted for $1.19 \%$ of the variation and, as with the first component, displayed no segregation. The specimen that was segregated from the others by the second component is MZUSP 33751, whose karyotype had been pre-

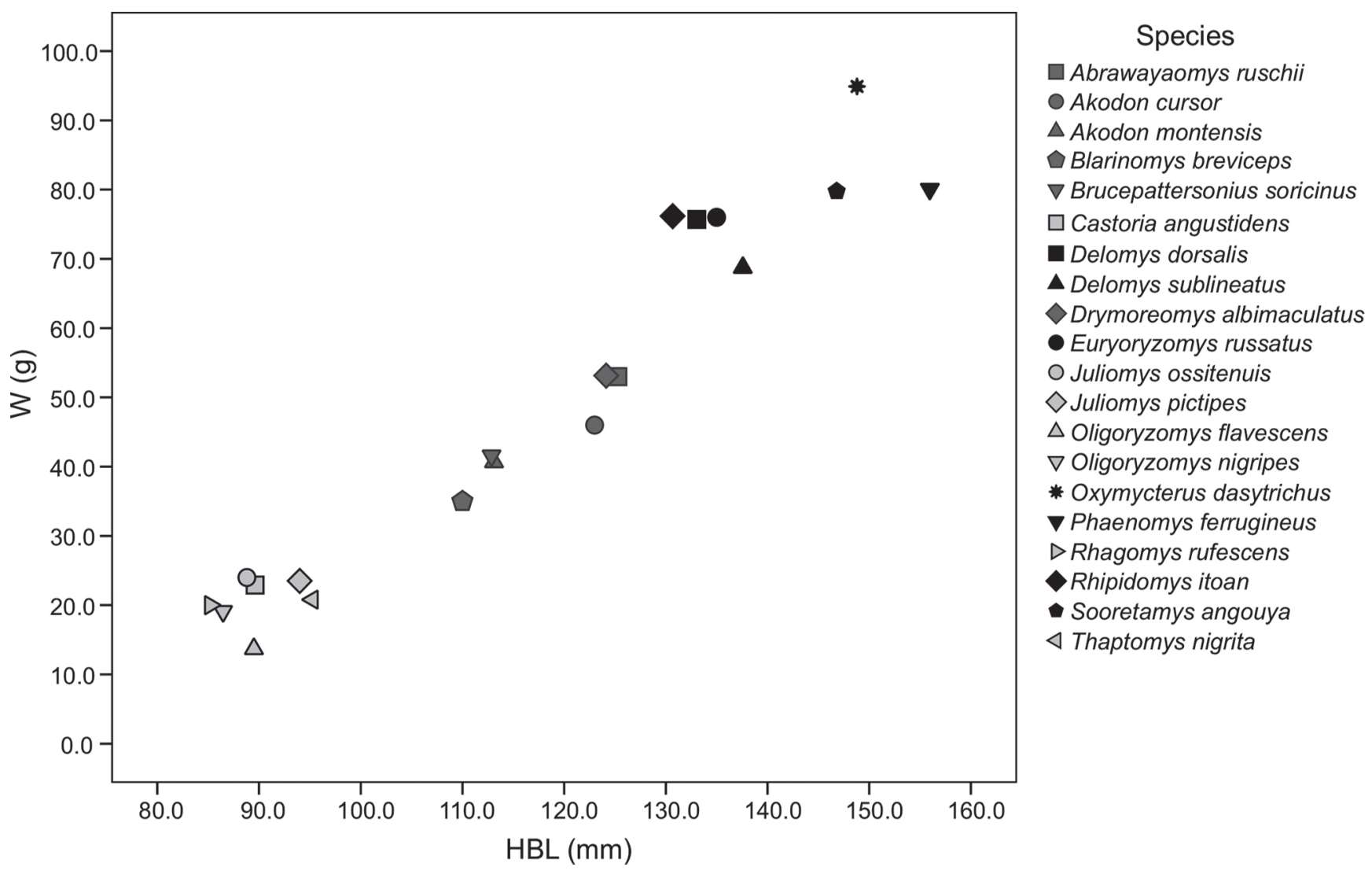

Figure 12. Biplot graph displaying the head and body length (HBL) and the weight (W) of 20 sigmodontine rodent species to determine their size categories. Light gray symbols represent small sized species, dark gray represent medium sized species, and black represent large sized species. 
viously analyzed and it was identified as $A$. cursor (Geise et al., 2005). Among the specimens that formed the large group in the PCA there are eight individuals with karyotype data and 10 with CYTB sequences (including individuals of all age classes and both sexes), and the information available corroborated their identification as $A$. montensis. The variables that most influenced the dispersion of individual scores in the second component, which is responsible for distinguishing the two species, were length of palatal bridge (LPB), least interorbital breadth (LIB), breadth of M1 (BM1) and breadth of braincase (BBC); the values of these and other variables in the first three main components are presented in Table 6. Therefore, this approach enabled us to recognize two species occurring at EEB: $A$. cursor (one specimen) and A. montensis (62 specimens).

Consistent with literature information, we found no external and/or cranial characteristics to differentiate between A. cursor and A. montensis in the samples from EEB. However, as observed in the results of PCA, a multivariate analysis of craniometric characters clearly distinguish between these two species. A comparison between the
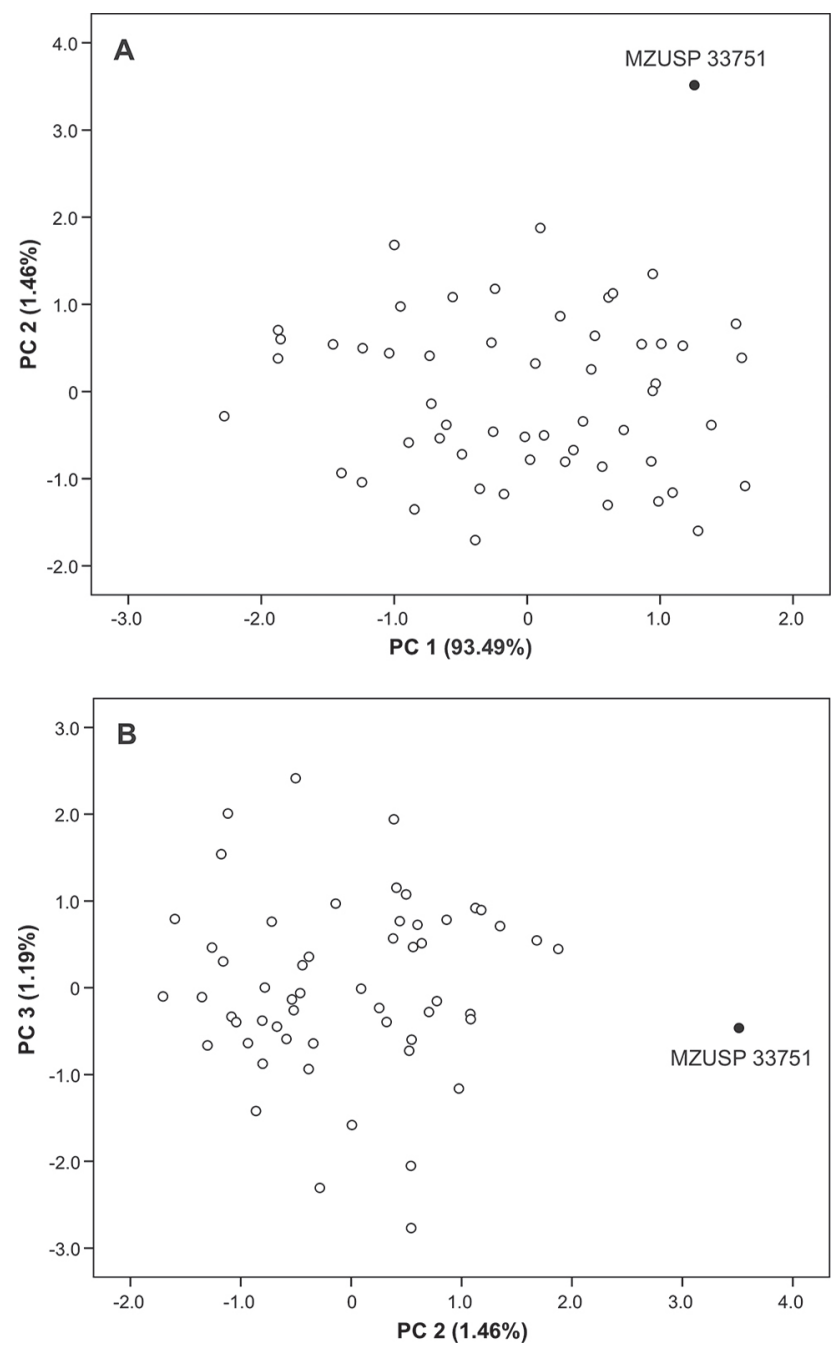

Figure 13. Scatterplot graph showing the projections of individual scores of Akodon montensis (white dots) and Akodon cursor (black dot) from a PCA analysis. (A) First principal component ( $P(1)$ versus second principal component $(P C 2)$ and $(B) P C 2$ versus third principal component $(P C 3)$.
Table 6. Scores of cranio-dental morphometric variables in the first three principal components from a PCA of Akodon from EEB. Variables that loaded higher on each component are indicated in bold.

\begin{tabular}{llll}
\hline \multicolumn{1}{c}{ Variables } & PC1 & PC2 & PC 3 \\
\hline CIL & $\mathbf{0 . 9 9 8}$ & 0.048 & -0.028 \\
CZL & $\mathbf{0 . 9 9 2}$ & 0.059 & -0.041 \\
LD & $\mathbf{0 . 9 8 0}$ & -0.024 & -0.040 \\
LIF & 0.911 & -0.164 & -0.052 \\
BIF & 0.811 & 0.116 & 0.067 \\
LM & 0.395 & 0.157 & -0.047 \\
BM1 & 0.542 & $\mathbf{0 . 2 7 6}$ & 0.040 \\
ID & 0.921 & 0.079 & -0.037 \\
IW & 0.835 & 0.184 & $-\mathbf{0 . 1 9 5}$ \\
LN & $\mathbf{0 . 9 6 3}$ & -0.240 & 0.073 \\
BR & 0.778 & 0.016 & 0.029 \\
BZP & 0.835 & 0.008 & 0.064 \\
LIB & 0.509 & $\mathbf{0 . 3 1 2}$ & 0.192 \\
OFL & 0.952 & 0.000 & -0.021 \\
LPB & 0.644 & $\mathbf{0 . 3 7 0}$ & 0.018 \\
BBP & 0.912 & 0.064 & 0.084 \\
HBC & 0.742 & -0.029 & $\mathbf{0 . 4 3 8}$ \\
BBC & 0.701 & $\mathbf{0 . 2 5 9}$ & $\mathbf{0 . 6 3 1}$ \\
OCB & 0.747 & 0.146 & $\mathbf{0 . 2 7 5}$ \\
\hline
\end{tabular}

cranio-dental measurements of adults of $A$. cursor and A. montensis are presented in Table 7. Even though only one $A$. cursor specimen was identified in the EEB sample, the values of some variables obtained for this species are higher than the highest values obtained for $A$. montensis; this corroborates the craniometric differentiation of these species (Gonçalves et al., 2007; Geise, 2012). The most significant and informative variables were: interorbital width, for which $A$. montensis presented mean value of $4.99 \mathrm{~mm}$ and maximum value of $5.30 \mathrm{~mm}$, while the value obtained from the EEB specimen of $A$. cursor was $5.38 \mathrm{~mm}$; length of the palatine bridge, which ranged from 3.14 to $4.05 \mathrm{~mm}$, with mean value of $3.62 \mathrm{~mm}$ in A. montensis, while the observed value in $A$. cursor was $4.09 \mathrm{~mm}$; length of the upper molar series, with mean value of $4.19 \mathrm{~mm}$ in $A$. montensis, with only one specimen showing greater value $(4.45 \mathrm{~mm})$ than the value obtained for A. cursor (4.43 mm); and width of $\mathrm{M} 1$, for which $A$. montensis presented mean value of $1.18 \mathrm{~mm}$ and maximum value of $1.28 \mathrm{~mm}$, and the observed value in $A$. cursor was $1.30 \mathrm{~mm}$.

\section{Akodon cursor (Winge 1887)}

Voucher material: $\mathrm{n}=1$; male: MZUSP 33751 .

Specimen identification: Medium size (see body measurements on Table 7). Dorsal pelage soft and dense, brown colored speckled with golden yellow, darker near the dorsal midline and on the rump; dorsal guard hairs long (length about $12 \mathrm{~mm}$ ), with the basal half narrower, black in color, and the apical half wider, brownish; dorsal cover hairs medium (length about $10 \mathrm{~mm}$ ), narrower basally (two thirds) and wider apically (one third), grayish 
Table 7. Body and cranio-dental morphometric values ( $\mathrm{mm})$, and weight $(\mathrm{g})$ of Akodon cursor from EBB, and descriptive statistics (mean \pm standard deviation, range, and sample size) of Akodon montensis from EBB. All measured specimens were adults.

\begin{tabular}{lcc}
\hline & Akodon cursor (1 male) & Akodon montensis (18 males and 15 females) \\
\hline HBL & 123.0 & $113.11 \pm 11.81(89.5-146.0) 28$ \\
TL & {$[86.0]$} & $92.63 \pm 7.84(80.0-115.0) 25$ \\
HF2 & 24.0 & $23.58 \pm 2.63(19.0-34.5) 28$ \\
HF1 & 26.0 & $25.32 \pm 2.48(21.0-36.0) 28$ \\
EL & 15.5 & $18.92 \pm 1.94(15.7-23.0) 28$ \\
W & 46.0 & $40.75 \pm 11.46(19.0-64.0) 26$ \\
GSL & 29.92 & $29.06 \pm 1.19(26.41-31.04) 33$ \\
CIL & 27.97 & $26.34 \pm 1.20(23.78-28.35) 33$ \\
CZL & 20.53 & $19.3 \pm 0.87(17.39-20.74) 33$ \\
LD & 8.03 & $7.76 \pm 0.47(6.90-8.60) 33$ \\
LIF & 6.39 & $6.51 \pm 0.39(5.91-7.26) 33$ \\
BIF & 2.51 & $2.44 \pm 0.18(2.16-2.85) 33$ \\
LM & 4.43 & $4.19 \pm 0.13(3.87-4.45) 33$ \\
BM1 & 1.30 & $1.18 \pm 0.05(1.07-1.28) 33$ \\
ID & 1.54 & $1.40 \pm 0.07(1.26-1.53) 33$ \\
IW & 1.87 & $1.53 \pm 0.11(1.25-1.74) 33$ \\
LN & 11.29 & $11.51 \pm 0.69(9.97-12.77) 33$ \\
BR & 3.68 & $3.78 \pm 0.20(3.37-4.21) 33$ \\
RL & 11.00 & $11.33 \pm 0.64(9.87-12.53) 33$ \\
ZB & 15.00 & $14.19 \pm 0.73(12.94-15.68) 33$ \\
BZP & 2.67 & $2.51 \pm 0.17(2.28-2.90) 33$ \\
LIB & 5.38 & $4.99 \pm 0.15(4.67-5.30) 33$ \\
OFL & 9.35 & $9.16 \pm 0.32(8.46-9.91) 33$ \\
PL & 11.90 & $11.69 \pm 0.55(10.58-12.85) 33$ \\
LPB & 4.09 & $3.62 \pm 0.26(3.14-4.05) 33$ \\
BBP & 3.42 & $2.98 \pm 0.17(2.67-3.41) 33$ \\
HBC & 8.64 & $8.53 \pm 0.25(8.12-9.03) 33$ \\
BBC & 12.84 & $12.50 \pm 0.31(11.89-13.29) 33$ \\
IPB & 6.58 & $6.76 \pm 1.03(4.32-8.46) 33$ \\
IPL & 1.59 & $1.71 \pm 0.41(0.95-2.91) 33$ \\
OCB & 6.81 & $6.39 \pm 0.16(6.04-6.78) 33$ \\
\hline & &
\end{tabular}

colored with the apical third composed by three bands of color, an inferior band dark gay, a subterminal band yellow and a dark apical band; dorsal wool hairs short (length about 7,5 mm), thinner, blackish colored with a brownish apex. Lateral pelage brownish slightly lighter than dorsum; limit between lateral and ventral color very subtle. Ventral pelage color predominantly ocher; individual ventral hairs with basal portion (two thirds) gray colored and apical portion (one third) ochraceous colored. Mystacial vibrissae short, a few hairs reaching the base of the pinnae when laid back. Pinnae with medium size and rounded; covered internally and externally with numerous hairs, brownish colored. Dorsal surface of manus covered by brown hairs; digits with short claws (claw of dIII $=2.0 \mathrm{~mm} ; \mathrm{n}=1$ ). Pes moderately long and narrow, dorsally covered by hairs usually with basal portion brown and apical portion whitish; dIII similar in length to dII and dIV; digits with medium long claws (claw of $\mathrm{dIII}=3.5 \mathrm{~mm} ; \mathrm{n}=1$ ); tufts of ungual hairs long, extending beyond the claws, white colored. Tail shorter than head and body; covered by short hairs, with length of one to two scales at the basal portion on dorsal surface and longer than two scales on ventral surface; scales arranged in approximately 17 transverse rows per centimeter at the base of the tail $(n=1)$; dorsal tail color dark brown, with dark brown hairs, and ventral tail color dark brown slightly lighter especially at the base, with predominantly whitish hairs; tail generally unicolor.

Skull elongated and narrow (Fig. 14; Table 7). Rostrum long. Nasals anteriorly long, extending beyond the superior incisors, and posteriorly short, not extending beyond the maxillary-frontal-lacrimal suture; with posterior margin rounded. Premaxillae short posteriorly, ending collinearly to nasals. Lacrimal with equal contact with the maxillary and frontal. Zygomatic notch deep. Interorbital region hourglass-shaped with supraorbital margins rounded anteriorly and slightly sharp posteriorly. Frontosquamosal suture not collinear with frontoparietal suture. Braincase small and rounded, without temporal crests. Parietal entirely restricted to the dorsal surface of the braincase. Zygomatic plate broad with anterodorsal margin produced as a sharp corner, conspicuously anterior to superior maxillary root of zygoma; posterior margin of zygomatic plate collinear to M1 alveolus. Jugal present and large, maxillary and squamosal processes of zygomatic arch not overlapping. Anterior opening of alisphenoid canal present. Alisphenoid strut present, buccinator-masticatory foramen and accessory oval foramen separated. Squamosal-alisphenoid groove present on braincase lateral view, in the squamosal and alisphenoid bones; sphenofrontal foramen present; posterior opening of alisphenoid canal large; stapedial foramen present and large in the ectotympanic bullae, on the petrosal-ectotympanic suture (stapedial and carotid circulatory pattern 1; Voss, 1988). Subsquamosal fenestra present. Mastoid completely ossified on one side and with a diminutive pit in the dorsal contact with the exoccipital border on other side. Incisive foramina long, posterior margin surpassing the anterior face of $\mathrm{M} 1$; anterior portion of incisive foramina narrow and acute, central region broad and posterior region as broader as central region. Palate short and wide, with the central region deeper; lateral excavations shallow and one central foramen present on each side; posterolateral palatal pits present as one large foramen on the right side and two small foramina on the left side. Mesopterygoid fossa surpassing the posterior face of $M 3$; sphenopalatine vacuities present but reduced as narrow openings, anterior to basisphenoid-presphenoid suture. Parapterygoid plate narrower than the middle portion of mesopterygoid fossae. Auditory bullae small; exposed flange of periotic extending to internal carotid canal. Mandible elongated, with ramus moderately deep (Fig. 14). Superior and posterior notches moderately deep. Capsular process of lower incisor alveolus present but reduced as a slight rounded elevation. Superior and inferior masseteric ridges with anterior portion conjoined as single crest. Upper incisors opisthodont. Maxillary toothrows parallel. M1 with anterocone divided into labial and lingual conules by the anteromedian flexus; anteroloph present but reduced as a projection of anterolabial conule; protostyle present; mesoloph present but reduced as a projection of paracone; enterostyle present. $\mathrm{m} 1$ with anteroconid 


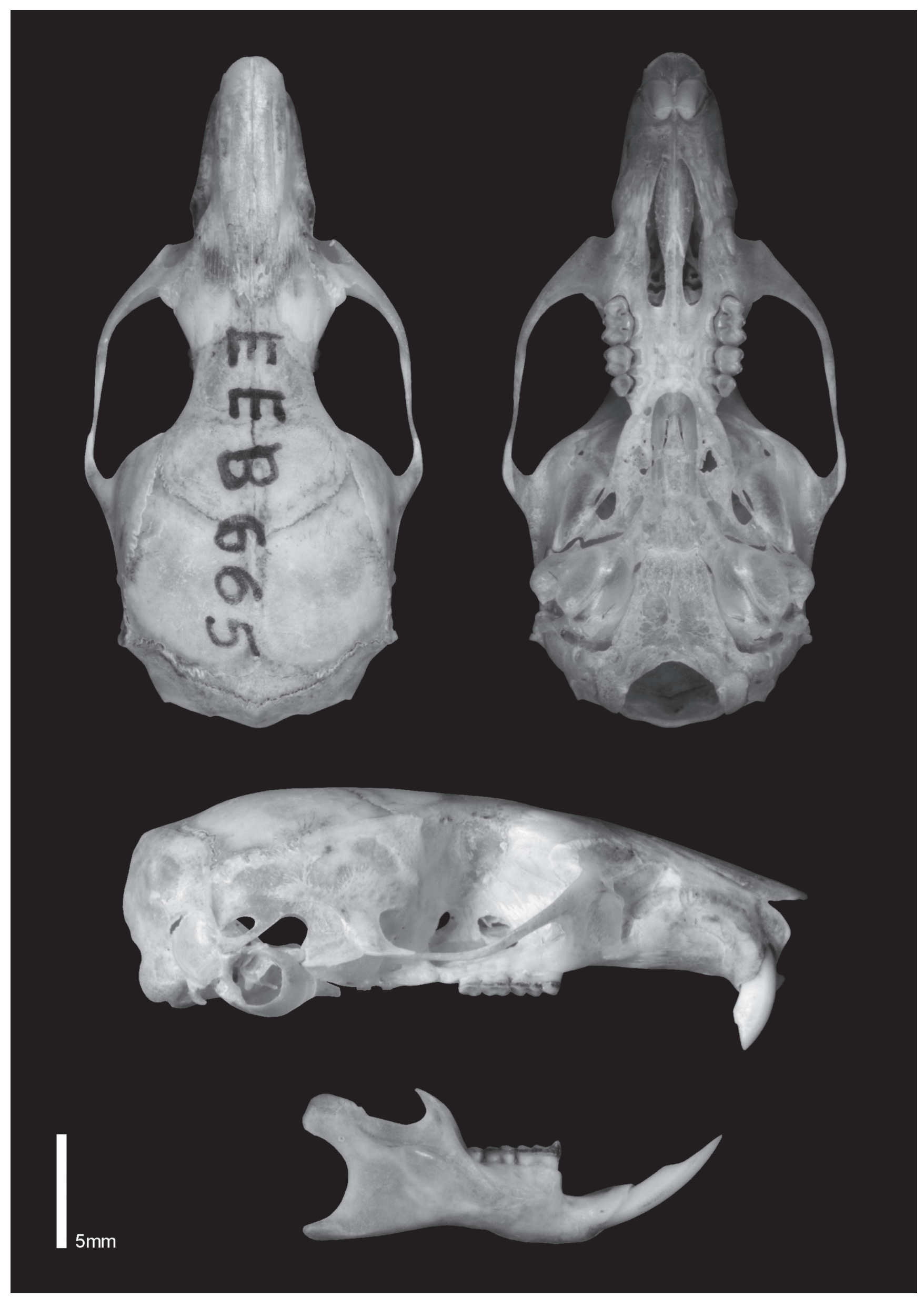

Figure 14. Skull and mandible of Akodon cursor (MZUSP 33751; male; GSL = $29.92 \mathrm{~mm}$ ). Skull on dorsal, ventral, and lateral view, and mandible on lateral view. 
divided into labial and lingual conulids by the anteromedian flexid; anterolophid absent; protostylid present; mesolophid present but reduced; ectostylid present.

Field observations: Only one specimen of $A$. cursor was identified in the EEB samples. It is a male with scrotal testes, collected on December, 2003, using conventional traps. This single specimens of $A$. cursor collected at EEB compared with the large series of $A$. montensis may result from the altitudinal segregation that occurs when these two species are found in sympatry in mountainous areas of the AF. According to Geise et al. (2005), when this happens $A$. cursor is found from sea level to altitudes around $1,000 \mathrm{~m}$, whereas $A$. montensis predominates above $800 \mathrm{~m}$. Therefore, the dominance of $A$. montensis in EEB samples was expected, as the altitude there varies between 1,100 and 2,000 m.

\section{Akodon montensis Thomas 1913}

Voucher material: $\mathrm{n}=62$; female: MZUSP 33681, 33685, 33686, 33688, 33692, 33695, 33697, 33698, 33699, 33702, 33706, 33761, 33766, 33769, 33779, 34890; uncatalogued: EBM 007, 016, 153, 212; EEB 539, 545, 563, 651, 670, 676, 678, 680, 1002, 1004, 1009, 1016; male: MZUSP 33679, 33682, 33683, 33687, 33691, 33700, 33704, 33759, 33764, 33767, 33770; uncatalogued: EBM 031, 033, 068, 234, 255; EEB 540, 553, 560, 574, 695, 703, 713, 813, 823, $843,903,922,1005,1007$.

Specimens identification: Medium size (see body measurements on Table 7). Dorsal pelage soft and moderately dense, brown speckled with dark golden yellow hairs, slightly darker on young and subadult specimens; dorsal guard hairs long (length ranging from 12 to $13.5 \mathrm{~mm}$ ), with the basal half narrower, gray in color, and the apical half wider, dark gray; dorsal cover hairs medium (length ranging from 9 to $11 \mathrm{~mm}$ ), narrower basally (two thirds) and wider apically (one third), grayish colored with the apical third composed by three bands of color, a basal band dark gay, a subterminal band yellow and a dark apical band; dorsal wool hairs short (length ranging from 8 to $9 \mathrm{~mm}$ ), thinner, dark gray colored with a subterminal band yellow and dark apex. Lateral pelage brownish lighter than dorsum, with yellow hairs more conspicuous and subtly grayish on young and subadult specimens; limit between lateral and ventral color very subtle, almost imperceptible in some specimens. Ventral pelage color overall ocher, grayish on young specimens and orange ocher on adult specimens; individual ventral hairs with basal portion (two thirds) light gray colored and apical portion (one third) ocher or orange ocher colored. Mystacial vibrissae short, a few hairs reaching the base of the pinnae when laid back. Pinnae with medium size and rounded; covered internally with numerous hairs, principally close to the apical portion, dark brown colored on the basal half and yellowish on the apical half, and with less numerous hairs externally, mostly grayish colored. Dorsal surface of manus covered by hairs with gray base and whitish top; digits with short or medium claws (claw of dIII with length ranging from 1.5 to $2.4 \mathrm{~mm} ; \mathrm{n}=3$ ). Pes moderately long and narrow, dorsally covered by hairs usually gray colored on base and whitish on top; dIII similar in length to dII and dIV; digits with medium claws (claw of dIII about $3.0 \mathrm{~mm}$ longer; $\mathrm{n}=3$ ); tufts of ungual hairs dense and long, extending beyond the claws, hairs with the basal portion gray and apical portion whitish. Tail shorter than head and body $(83.6 \% \pm 6.2$ of head and body length, ranging from 71.3 to $97.5 \%$; $n=46$ ); covered by short hairs, with length of two scales at the basal portion on both surfaces; scales arranged in 16 to 18 transverse rows per centimeter, at the base of the tail, between 15 to 18 rows per $\mathrm{cm}$ on the central portion of the tail, and between 20 and 22 rows on the apical centimeter of the tail $(n=3)$; overall dorsal tail color dark brown, with dark brown hairs, and ventral tail color dark brown slightly lighter especially at the base, with predominantly whitish hairs; tail generally unicolor.

Skull elongated and narrow (Fig. 15; Table 7). Rostrum long. Nasals anteriorly long, extending beyond the superior incisors, and posteriorly short, not extending beyond the maxillary-frontal-lacrimal suture $(87.9 \%)$ or long, extending beyond the maxillary-frontal-lacrimal suture $(12.1 \%)(n=33)$; with posterior margin rounded $(48.5 \%)$, sharp (30.3\%) or mostly rounded but with the terminal portion abruptly thin $(21.2 \%)(n=33)$. Premaxillae posteriorly short, ending anteriorly to nasals (78.8\%) or ending collinear to nasals $(21.2 \%)(n=33)$. Lacrimal with equal contact with maxillary and frontal $(69.7 \%)$ or in contact mainly with maxillary $(30.3 \%)(n=33)$. Zygomatic notch deep. Interorbital region hourglass in shape with squared supraorbital margins (52.9\%), or with rounded anteriorly and squared posteriorly supraorbital margins (44.1\%), or with conspicuously beaded supraorbital margins (2.9\%) $(n=34)$. Frontosquamosal suture not collinear with frontoparietal suture $(66.7 \%)$ or collinear $(33.3 \%)$ ( $n=33)$. Braincase small and rounded, without temporal crests. Parietal entirely restricted to the dorsal surface of the braincase $(71.9 \%)$ or partial expanded to the lateral surface of the braincase $(28.1 \%)(n=32)$. Zygomatic plate broad with anterodorsal margin smoothly rounded and conspicuously anterior to superior maxillary root of zygoma (50.0\%), or anterodorsal margin produced as a sharp corner $(47.1 \%)$, or produced as a prominent spinous process $(2.9 \%)(n=34)$; posterior margin of zygomatic plate situated approximately even with the alveolus of M1 (94.1\%) or situated anterior to the alveolus of M1 (5.9\%) $(n=34)$. Jugal present and large, maxillary and squamosal processes of zygomatic arch not overlapping (90.3\%), or small, maxillary and squamosal processes overlapping, but not in contact $(9.7 \%)(n=31)$. Anterior opening of alisphenoid canal present. Alisphenoid strut present, buccinator-masticatory foramen and accessory oval foramen separate $(97.1 \%)$, or absent, buccinator-masticatory foramen and accessory oval foramen confluent (2.9\%) $(n=34)$. Squamosal-alisphenoid groove present on braincase lateral view, in the squamosal and alisphenoid bones; sphenofrontal foramen present; posterior opening of alisphenoid canal large; stapedial foramen present and large 


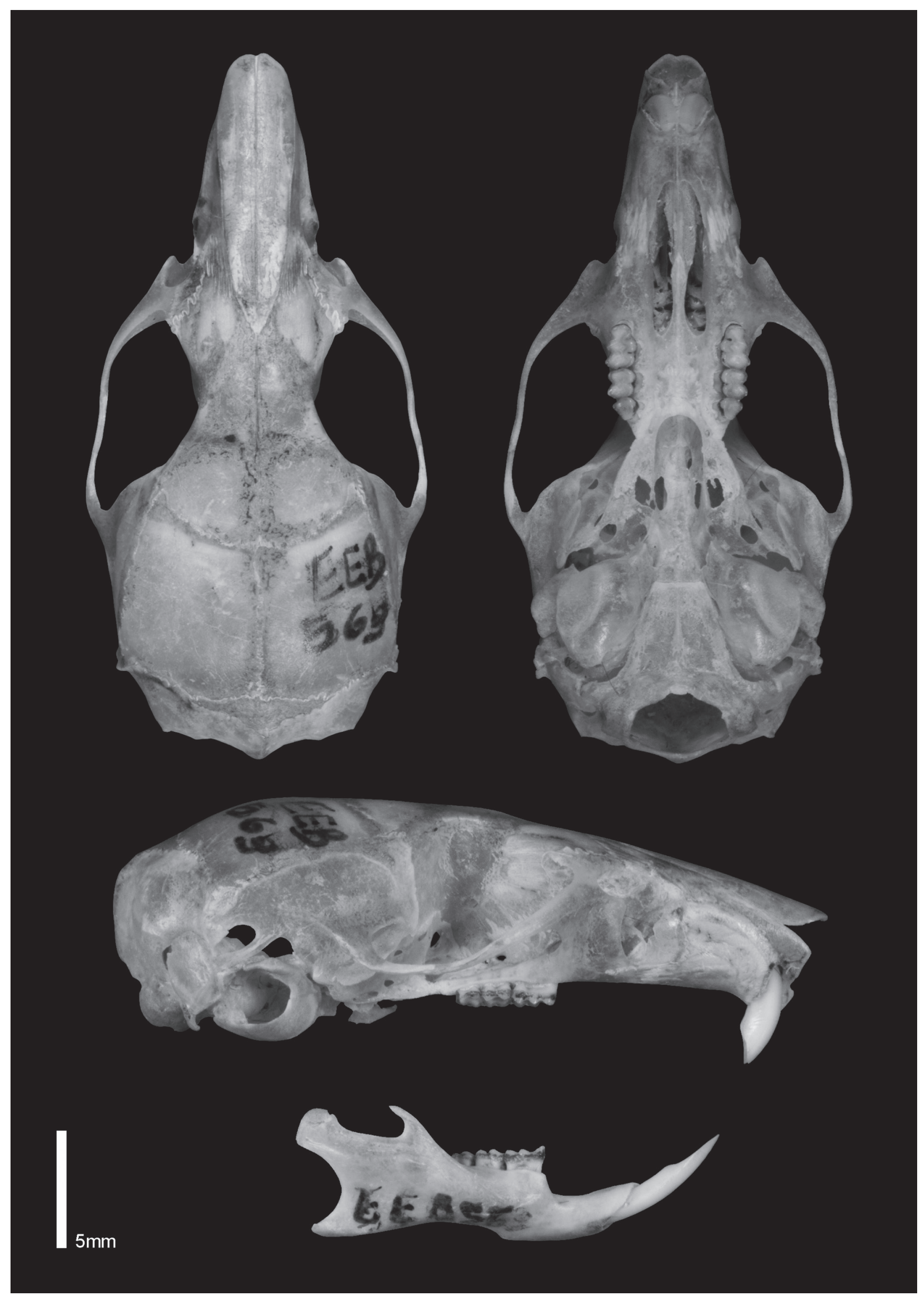

Figure 15. Skull and mandible of Akodon montensis (EEB 563; female; GSL $=30.45 \mathrm{~mm}$ ). Skull on dorsal, ventral, and lateral view, and mandible on lateral view. 
in the ectotympanic bullae, on the petrosal-ectotympanic suture (stapedial and carotid circulatory pattern 1; Voss, 1988). Subsquamosal fenestra present. Mastoid with conspicuous fenestra. Incisive foramina long, posterior margin surpassing the anterior face of $\mathrm{M} 1$ and reaching the anterocone (2.9\%), reaching the protoflexus $(50.0 \%)$, reaching the protocone (44.1\%), or reaching the hipoflexus $(2.9 \%)(n=34)$; anterior portion of incisive foramina narrow and acute, central region slightly broader than ends (principally on young specimens) or with same width of posterior region. Palate short and wide, with the central region deeper; lateral excavations shallow and one central foramen present on each side; posterolateral palatal pits present as one small pit on each side of posterior palate $(48.5 \%)$ or present as more than one pit on at least one side of posterior palate $(51.5 \%)(n=33)$. Mesopterygoid fossa reaching the posterior face of M3 $(61.8 \%)$, surpassing the posterior face of M3 (29.4\%) or not reaching the posterior face of M3 (8.8\%) ( $n=34)$; sphenopalatine vacuities present but reduced as narrow openings, anterior to basisphenoid-presphenoid suture (60.0\%), or vacuities present as large openings along the presphenoid reaching basisphenoid (6.7\%), or vacuities absent, mesopterygoid roof completely ossified (33.3\%) $(n=30)$. Parapterygoid plate narrower than the middle portion of mesopterygoid fossae $(67.7 \%)$, with similar width to the middle portion of mesopterygoid fossae (19.4\%), or wider than the middle portion of mesopterygoid fossae (12.9\%) $(n=31)$. Auditory bullae small, exposed flange of periotic extends to internal carotid canal (63.6\%), or intermediate, exposed wedge of periotic smaller and not contributing to wall of carotid canal $(36.4 \%)(n=33)$.

Mandible elongated, with ramus slightly low (Fig. 15). Superior and posterior notches moderately deep. Capsular process of lower incisor alveolus present but reduced as a slight rounded elevation (82.4\%), present and well developed (2.9\%), or absent $(14.7 \%)(n=34)$. Superior and inferior masseteric ridges with anterior portion conjoined as single crest $(67.6 \%)$ or superior and inferior masseteric ridges converge anteriorly as an opening "V" shaped (32.4\%) ( $n=34)$. Upper incisors opisthodont. Maxillary toothrows parallels. M1 with anterocone divided into labial and lingual conules by the anteromedian flexus; anteroloph present but reduced as a projection of anterolabial conule; mesoloph present but reduced as a projection of paracone. $\mathrm{m} 1$ with anteroconid divided into labial and lingual conulids by the anteromedian flexid; anterolophid absent; protolophid and protostylid present and well developed; mesolophid present but reduced; ectolophid and ectostylid absent $(55.6 \%)$ or present $(44.4 \%)(n=27)$.

Ontogenetic and sexual variation: Ontogenetic and sex-related variation were investigated in $A$. montensis from EEB, based on cranio-dental variables. For the ontogenetic analysis, specimens were classified in five age classes (ACs) and subsequently subjected to ANOVA followed by Tukey's post-hoc test. The ANOVA showed that 22 out of 25 variables tested varied significantly with age (Table 8 ). The Tukey's test showed that individuals in
AC1 differ from those in AC2 in 16 variables, individuals in AC2 differ from AC3 in 12 variables, individuals in $A C 3$ differ from AC4 in 14 variables, and individuals in AC4 are statistically the same as individuals in $A C 5$, since no variable differed significantly between these two age classes (Table 8).

Consistent ontogenetic differences have been documented in some Akodontini rodents. For example, studying the boliviensis group of the genus Akodon, Myers et al. (1990) revealed the presence of variation among all age classes in most variables. Only between classes 4 and 5 they observed a decrease in the number of variables with significant variation. Libardi (2013), analyzing this variation in samples of Necromys lasiurus, also found significant values among all classes, which decreased, in females, between classes 4 and 5 . In general, the results obtained for A. montensis collected at EEB corroborate these studies, and the fact that variation between age-classes decreases in the older classes, AC4 and AC5. Both studies mentioned above, in addition to the contribution of Ventura et al. (2000) using populations of the Akodontini Necromys urichi and Microxus bogotensis from Venezuela, highlight a continuous increase in the values of variables with age, which was also observed in A. montensis from EEB.

The lack of ontogenetic variation in the measurements of the upper molars, as observed for the LM in this study, is common in sigmodontine rodents (e.g., Voss, 1991; Brandt \& Pessôa, 1994; Prado \& Percequillo, 2011). However, these variables vary more in some Akodontini, at least in the lower age classes (e.g., Myers et al., 1990; Libardi, 2013).

The variables IPB and IPL have a different variation pattern from all other measurements, with higher AC2 means and random variation (higher and lower means) among the age classes; the lack of age-related differences in the interparietal breadth was also observed in the species of the tribe Oryzomyini, Aegialomys xanthaeolus (Prado \& Percequillo, 2011), and between classes 1 and 2 in the species of the tribe Akodontini, Necromys lasiurus (Libardi, 2013).

To test for sexual dimorphism, specimens were reclassified into four age-groups based on the results of the previous ontogenetic analysis: juveniles, $A C 1$; subadults, AC2; young adults, AC3; and old adults, AC4 plus AC5. A Student's t-test comparing craniometric values between males and females of each age class was conducted. The results, in Tables 9 and 10, show that in juveniles only three (IW, RL, BBP) of the 24 variables tested differed significantly between the sexes; in subadults, six (GSL, CIL, $\mathrm{RL}, \mathrm{PL}, \mathrm{BBP}$ and $\mathrm{HBC}$ ) of the 25 variables presented sexual dimorphism; in young adults, significant sexual differences were observed only in one variable (IPB); and in old adults, four (CIL, LD, BIF and ID) of the 25 measurements were significantly different between the sexes.

This result differs from those of Geise et al. (2005), who studied sexual dimorphism in populations of $A$. montensis from several localities and found that sexual dimorphism was present in 14 out of 20 craniometric variables. However, when pulling together specimens from distinct localities (as performed by Geise et al., 2005) another 
Table 8. Results of Kolmogorov-Smirnov test, Analysis of Variance (ANOVA), and Tukey's post-hoc test, and sample size in each age class for 25 cranio-dental morphometric variables measured in Akodon montensis from EEB. Statistically significant values $(P<0.05)$ are indicated in bold.

\begin{tabular}{|c|c|c|c|c|c|c|c|c|c|c|c|c|c|c|c|c|c|c|}
\hline & \multirow{2}{*}{ K-S Sig. } & \multicolumn{2}{|c|}{ ANOVA } & \multicolumn{10}{|c|}{ Tukey } & \multicolumn{5}{|c|}{$\mathrm{N}$} \\
\hline & & $\mathbf{F}$ & Sig. & 1 vs. 2 & 1 vs. 3 & 1 vs. 4 & 1 vs. 5 & 2 vs. 3 & 2 vs. 4 & 2 vs. 5 & 3 vs. 4 & 3 vs. 5 & 4 vs. 5 & 1 & 2 & 3 & 4 & 5 \\
\hline GSL & 0.610 & 61.208 & 0.000 & 0.000 & 0.000 & 0.000 & 0.000 & 0.002 & 0.000 & 0.000 & 0.000 & 0.004 & 0.996 & 10 & 15 & 12 & 17 & 4 \\
\hline CIL & 0.770 & 63.193 & 0.000 & 0.000 & 0.000 & 0.000 & 0.000 & 0.002 & 0.000 & 0.000 & 0.000 & 0.001 & 0.890 & 10 & 15 & 12 & 17 & 4 \\
\hline CZL & 0.880 & 51.783 & 0.000 & 0.000 & 0.000 & 0.000 & 0.000 & 0.029 & 0.000 & 0.001 & 0.000 & 0.001 & 0.918 & 10 & 15 & 12 & 17 & 4 \\
\hline LD & 0.887 & 73.904 & 0.000 & 0.000 & 0.000 & 0.000 & 0.000 & 0.000 & 0.000 & 0.000 & 0.000 & 0.001 & 0.556 & 13 & 15 & 12 & 17 & 4 \\
\hline LIF & 0.914 & 45.434 & 0.000 & 0.000 & 0.000 & 0.000 & 0.000 & 0.113 & 0.000 & 0.000 & 0.016 & 0.003 & 0.391 & 13 & 15 & 12 & 17 & 4 \\
\hline BIF & 0.877 & 18.665 & 0.000 & 0.077 & 0.000 & 0.000 & 0.000 & 0.009 & 0.000 & 0.026 & 0.734 & 0.959 & 1.000 & 13 & 15 & 12 & 17 & 4 \\
\hline LM & 0.929 & 0.849 & 0.501 & - & - & - & - & - & - & - & - & - & - & 9 & 14 & 12 & 17 & 4 \\
\hline BM1 & 0.383 & 3.511 & 0.013 & 0.765 & 0.708 & 0.011 & 0.189 & 1.000 & 0.168 & 0.593 & 0.294 & 0.686 & 1.000 & 13 & 15 & 12 & 17 & 4 \\
\hline ID & 0.470 & 50.788 & 0.000 & 0.002 & 0.000 & 0.000 & 0.000 & 0.000 & 0.000 & 0.000 & 0.006 & 0.127 & 1.000 & 10 & 15 & 12 & 17 & 4 \\
\hline IW & 0.464 & 30.102 & 0.000 & 0.428 & 0.000 & 0.000 & 0.000 & 0.002 & 0.000 & 0.000 & 0.034 & 0.008 & 0.488 & 11 & 15 & 12 & 17 & 4 \\
\hline LN & 0.537 & 61.665 & 0.000 & 0.000 & 0.000 & 0.000 & 0.000 & 0.009 & 0.000 & 0.000 & 0.000 & 0.055 & 0.997 & 11 & 15 & 12 & 17 & 4 \\
\hline BR & 0.671 & 11.853 & 0.000 & 0.017 & 0.003 & 0.000 & 0.000 & 0.935 & 0.039 & 0.010 & 0.307 & 0.049 & 0.491 & 10 & 15 & 12 & 17 & 4 \\
\hline $\mathbf{R L}$ & 0.770 & 78.681 & 0.000 & 0.000 & 0.000 & 0.000 & 0.000 & 0.000 & 0.000 & 0.000 & 0.000 & 0.000 & 0.465 & 11 & 15 & 12 & 17 & 4 \\
\hline ZB & 0.779 & 47.224 & 0.000 & 0.002 & 0.000 & 0.000 & 0.000 & 0.136 & 0.000 & 0.000 & 0.000 & 0.001 & 1.000 & 9 & 14 & 12 & 17 & 4 \\
\hline BZP & 0.984 & 19.172 & 0.000 & 0.083 & 0.000 & 0.000 & 0.000 & 0.158 & 0.000 & 0.005 & 0.066 & 0.281 & 0.999 & 13 & 15 & 12 & 17 & 4 \\
\hline LIB & 0.810 & 3.889 & 0.007 & 0.092 & 0.127 & 0.017 & 0.015 & 1.000 & 0.968 & 0.504 & 0.972 & 0.524 & 0.744 & 12 & 15 & 12 & 17 & 4 \\
\hline OFL & 0.278 & 46.036 & 0.000 & 0.000 & 0.000 & 0.000 & 0.000 & 0.027 & 0.000 & 0.000 & 0.002 & 0.027 & 0.992 & 10 & 15 & 12 & 17 & 4 \\
\hline PL & 0.665 & 65.975 & 0.000 & 0.000 & 0.000 & 0.000 & 0.000 & 0.000 & 0.000 & 0.000 & 0.002 & 0.003 & 0.707 & 13 & 15 & 12 & 17 & 4 \\
\hline LPB & 0.956 & 8.961 & 0.000 & 0.822 & 0.028 & 0.000 & 0.003 & 0.230 & 0.002 & 0.018 & 0.532 & 0.437 & 0.951 & 13 & 15 & 12 & 17 & 4 \\
\hline BBP & 0.551 & 56.905 & 0.000 & 0.000 & 0.000 & 0.000 & 0.000 & 0.014 & 0.000 & 0.000 & 0.000 & 0.033 & 0.938 & 12 & 15 & 12 & 17 & 4 \\
\hline $\mathrm{HBC}$ & 0.800 & 9.936 & 0.000 & 0.023 & 0.006 & 0.000 & 0.005 & 0.951 & 0.016 & 0.526 & 0.153 & 0.835 & 0.987 & 10 & 15 & 12 & 17 & 4 \\
\hline BBC & 0.952 & 8.512 & 0.000 & 0.013 & 0.001 & 0.000 & 0.166 & 0.833 & 0.108 & 1.000 & 0.695 & 0.942 & 0.464 & 10 & 15 & 12 & 17 & 4 \\
\hline IPB & 0.458 & 2.022 & 0.105 & - & - & - & - & - & - & - & - & - & - & 10 & 15 & 12 & 17 & 4 \\
\hline IPL & 0.800 & 0.285 & 0.887 & - & - & - & - & - & - & - & - & - & - & 10 & 15 & 12 & 17 & 4 \\
\hline$O C B$ & 0.726 & 14.518 & 0.000 & 0.014 & 0.002 & 0.000 & 0.001 & 0.925 & 0.000 & 0.329 & 0.014 & 0.687 & 0.899 & 10 & 15 & 12 & 17 & 4 \\
\hline
\end{tabular}

Table 9. Descriptive statistics (mean \pm standard deviation, and sample size) of 25 cranio-dental measurements of young and subadult male and female specimens of Akodon montensis from EEB, and results of Student's t-test and its value of significance. Statistically significant values $(P<0.05)$ are indicated in bold.

\begin{tabular}{|c|c|c|c|c|c|c|c|c|}
\hline & \multicolumn{4}{|c|}{ Young } & \multicolumn{4}{|c|}{ Subadults } \\
\hline & Males & Females & $t$ & Sig. & Males & Females & $t$ & Sig. \\
\hline GSL & $23.98 \pm 0.14(2)$ & $24.88 \pm 1.16(8)$ & 2.120 & 0.068 & $27.07 \pm 0.88(7)$ & $26.13 \pm 0.67(8)$ & -2.357 & 0.035 \\
\hline CIL & $21.31 \pm 0.49(2)$ & $22.24 \pm 1.04(8)$ & 1.856 & 0.139 & $24.33 \pm 0.80(7)$ & $23.45 \pm 0.75(8)$ & -2.200 & 0.046 \\
\hline CZL & $15.93 \pm 0.28(2)$ & $16.58 \pm 0.80(8)$ & 1.093 & 0.306 & $17.97 \pm 0.67(7)$ & $17.48 \pm 0.61(8)$ & -1.486 & 0.161 \\
\hline LD & $5.77 \pm 0.34(4)$ & $6.09 \pm 0.33(9)$ & 1.592 & 0.140 & $6.89 \pm 0.35(7)$ & $6.55 \pm 0.27(8)$ & -2.115 & 0.054 \\
\hline LIF & $5.12 \pm 0.41(4)$ & $5.30 \pm 0.31(9)$ & 0.884 & 0.395 & $6.07 \pm 0.23(7)$ & $5.82 \pm 0.22(8)$ & -2.133 & 0.053 \\
\hline BIF & $1.95 \pm 0.10(4)$ & $2.02 \pm 0.12(9)$ & 0.988 & 0.345 & $2.23 \pm 0.17(7)$ & $2.11 \pm 0.16(8)$ & -1.349 & 0.200 \\
\hline LM & 4.35 & $4.07 \pm 0.11(8)$ & - & - & $4.12 \pm 0.10(6)$ & $4.19 \pm 0.17(8)$ & 0.860 & 0.407 \\
\hline BM1 & $1.13 \pm 0.05(4)$ & $1.13 \pm 0.03(9)$ & -0.188 & 0.854 & $1.15 \pm 0.05(7)$ & $1.15 \pm 0.07(8)$ & 0.000 & 1.000 \\
\hline ID & $1.09 \pm 0.06(2)$ & $1.11 \pm 0.08(8)$ & 0.350 & 0.736 & $1.24 \pm 0.09(7)$ & $1.19 \pm 0.07(8)$ & -1.058 & 0.309 \\
\hline IW & $1.17 \pm 0.08(3)$ & $1.27 \pm 0.05(8)$ & 2.763 & 0.022 & $1.36 \pm 0.13(7)$ & $1.26 \pm 0.10(8)$ & -1.563 & 0.142 \\
\hline LN & $8.41 \pm 0.38(3)$ & $9.00 \pm 0.45(8)$ & 2.017 & 0.074 & $10.38 \pm 0.63(7)$ & $10.04 \pm 0.47(8)$ & -1.217 & 0.245 \\
\hline BR & $3.33 \pm 0.10(3)$ & $3.42 \pm 0.14(7)$ & 1.075 & 0.314 & $3.72 \pm 0.14(7)$ & $3.55 \pm 0.22(8)$ & -1.848 & 0.087 \\
\hline RL & $8.45 \pm 0.16(3)$ & $9.09 \pm 0.47(8)$ & 2.278 & 0.049 & $10.18 \pm 0.42(7)$ & $9.70 \pm 0.38(8)$ & -2.358 & 0.035 \\
\hline ZB & $11.87 \pm 0.44(2)$ & $12.21 \pm 0.70(7)$ & 0.635 & 0.546 & $13.22 \pm 0.46(6)$ & $12.79 \pm 0.41(8)$ & -1.839 & 0.091 \\
\hline BZP & $2.02 \pm 0.08(4)$ & $2.11 \pm 0.19(9)$ & 0.938 & 0.368 & $2.35 \pm 0.16(7)$ & $2.17 \pm 0.21(8)$ & -1.829 & 0.090 \\
\hline LIB & $4.78 \pm 0.13(4)$ & $4.81 \pm 0.12(8)$ & 0.500 & 0.628 & $5.01 \pm 0.14(7)$ & $4.90 \pm 0.22(8)$ & -1.195 & 0.254 \\
\hline OFL & $7.67 \pm 0.11(2)$ & $7.94 \pm 0.37(8)$ & 0.996 & 0.348 & $8.67 \pm 0.34(7)$ & $8.41 \pm 0.34(8)$ & -1.449 & 0.171 \\
\hline PL & $9.42 \pm 0.31(4)$ & $9.71 \pm 0.47(9)$ & 1.140 & 0.278 & $10.71 \pm 0.31(7)$ & $10.32 \pm 0.36(8)$ & -2.199 & 0.047 \\
\hline LPB & $3.14 \pm 0.18(4)$ & $3.25 \pm 0.29(9)$ & 0.690 & 0.505 & $3.37 \pm 0.21(7)$ & $3.26 \pm 0.25(8)$ & -0.897 & 0.386 \\
\hline BBP & $2.31 \pm 0.03(3)$ & $2.43 \pm 0.15(9)$ & 2.467 & 0.035 & $2.75 \pm 0.17(7)$ & $2.55 \pm 0.09(8)$ & -2.907 & 0.012 \\
\hline HBC & $8.02 \pm 0.13(2)$ & $8.00 \pm 0.30(8)$ & -0.050 & 0.961 & $8.52 \pm 0.27(7)$ & $8.16 \pm 0.16(8)$ & -3.269 & 0.006 \\
\hline BBC & $11.77 \pm 0.16(2)$ & $11.88 \pm 0.41(8)$ & 0.363 & 0.726 & $12.48 \pm 0.26(7)$ & $12.18 \pm 0.37(8)$ & -1.664 & 0.122 \\
\hline IPB & $7.06 \pm 0.62(2)$ & $6.84 \pm 0.83(8)$ & -0.345 & 0.739 & $7.42 \pm 1.25(7)$ & $7.18 \pm 0.91(8)$ & -0.435 & 0.671 \\
\hline IPL & $1.95 \pm 0.04(2)$ & $1.65 \pm 0.32(8)$ & -1.243 & 0.249 & $1.82 \pm 0.34(7)$ & $1.74 \pm 0.19(8)$ & -0.563 & 0.583 \\
\hline$O C B$ & $6.00 \pm 0.23(2)$ & $5.99 \pm 0.23(8)$ & -0.062 & 0.952 & $6.30 \pm 0.20(7)$ & $6.14 \pm 0.16(8)$ & -1.736 & 0.106 \\
\hline
\end{tabular}


source of variation is added - the geographical variation - which could lead to inaccurate interpretation of sexual differences, as males and females from one region may be larger than individuals of both sex from other region.

Craniometric sexual dimorphism has been documented in different Akodontini species, at least in one age class, but in the same time it was not considered an important source of population variation. For example, Macêdo \& Mares (1987) found sexual dimorphism in 83\% of the measurements of Necromys lasiurus, and Myers et al. (1990) observed conspicuous sex differences in the boliviensis group of the genus Akodon using multivariate analysis; in these studies, however, these differences were not considered relevant, as the average values of the variables in question did not differ significantly between males and females. Furthermore, a consistent pattern was not observed in the dimorphic variables of the boliviensis group of Akodon.

The specimens from EEB fit in the latter case, as only two measurements were dimorphic in more than one age class: the $R L$, in juveniles and subadults, and the CIL, in subadults and old adults. The remaining variables were dimorphic in only one age class, demonstrating the random nature of dimorphism in this population. Given that the dimorphic variables did not coincide between the age groups, besides representing a very low percentage of the total measurements tested, particularly in adults, we consider that sexual dimorphism is punctual and not relevant in the sample of $A$. montensis from EEB. Agerelated variations, by contrast, are prominent in the population, as previously pointed out by Myers et al. (1990).

Field observations: $A$ total of $62 A$. montensis specimens were identified in the EEB samples. Of these, 42 were collected in December, 2003, 11 in April, 2004, one in May, 2004, two in December, 2004, and six in December, 2011. They were collected by conventional traps (48.4\%) and pitfall traps (51.6\%). In December 2003, 22 females were captured, nine of which showed signs of reproductive activity (perforated vagina, mammary glands visible or pregnancy) and eight without those signs (unperforated vaginas), and the reproductive condition of five females was not observed; also 18 males were trapped, 12 with scrotal testes and three with abdominal testes, in addition to three individuals whose reproductive state was not ascertained, and two specimens of undetermined sex. In April, 2004, four females were captured, three in reproductive activity and one without signs of it; and seven males, three with scrotal testes and two without information on their reproductive status. In December, 2011, four females were captured, none of which seemed to be in reproductive activity, and two males, both with scrotal testes. No information on reproductive activity was obtained for the specimens captured in May and December of 2004.

\section{Blarinomys breviceps (Winge 1887)}

Voucher material: $\mathrm{n}=5$; female: uncatalogued: EEB 776; male: uncatalogued: EEB 720, 854, 855, 858.
Specimens identification: Medium size (see body measurements on Table 11). Dorsal pelage dense and velvety, although slightly hispid, dark gray colored with brown shades; dorsal guard hairs long (length about $10 \mathrm{~mm}$ ), with the basal half narrower, light gray in color, and the apical half wider and flat, dark gray in color, apex thin; dorsal cover hairs medium (length about $8.5 \mathrm{~mm}$ ), with two basal thirds light gray and the apical third brownish dark gray; dorsal wool hairs short (length about $7 \mathrm{~mm}$ ), light gray colored with a brownish terminal band (about $1 / 6$ of hair length). Lateral pelage dark gray colored, similar to dorsum; limit between lateral and ventral color not conspicuous. Ventral pelage gray colored; individual ventral hairs with basal portion (3/4) gray in color and apical portion ( $1 / 4)$ brownish. Mystacial vibrissae short, not reaching the base of the pinnae when laid back. Pinnae very small and rounded, with a strip of dark gray hairs on the superior edge of external surface. Dorsal surface of manus covered by grayish brown hairs; digits with long claws (claw of dlll = $3.5 \mathrm{~mm} ; \mathrm{n}=1$ ). Pes short and relatively wide, dorsally covered by grayish brown hairs; dIII slightly longer than dII and dIV; digits with long claws (claw of dIII = $3.5 \mathrm{~mm} ; \mathrm{n}=1$ ); tufts of ungual hairs short and sparse, not extending beyond the claws, brownish colored. Tail notably shorter than head and body (ca. $45.5 \%$ of head and body length, ranging from 43.5 to $47.6 \% ; n=2$ ); tail covered by long hairs, with length of two to three scales at the basal portion on both surfaces; scales arranged in 18 transverse rows per centimeter at the base of the tail and in 17 rows on the apical centimeter of the tail $(n=1)$; dorsal and ventral tail color brown, with brown hairs; tail unicolor.

Skull short and wide (Fig. 16; Table 11). Rostrum long. Nasals posteriorly long, extending beyond the maxillary-frontal-lacrimal suture; with posterior margin sharp. Premaxillae posteriorly short, ending anteriorly to nasals. Lacrimal with equal contact with maxillary and frontal (three specimens) or in contact mainly with maxillary (one specimen). Zygomatic notch indistinguishable. Interorbital region very broad with smooth posterior constriction and with rounded supraorbital margins. Frontosquamosal suture collinear with frontoparietal suture. Braincase large, very wide and inflated; without temporal crests. Parietal entirely restricted to the dorsal surface of the braincase. Interparietal absent. Lambdoidal crests well developed and acute; anteriorly displaced and situated close to squamosal root of the zygomatic arch. Nasals and premaxillae projected anteriorly beyond incisors, forming a distinct and long rostral tube. Gnathic process well developed. Zygomatic plate narrow, short and slightly sloped posteriorly with anterodorsal margin rounded; posterior margin of zygomatic plate situated approximately even with the alveolus of M1. Masseteric tubercle developed. Jugal present and large, maxillary and squamosal processes of zygomatic arch not overlapping. Anterior opening of alisphenoid canal present but small. Alisphenoid strut present on both sides, buccinator-masticatory foramen and accessory oval foramen separate (two specimens), present in only one side (one specimen), or absent, buccinator-masticatory foramen 
Table 10. Descriptive statistics (mean \pm standard deviation, and sample size) of 25 cranio-dental measurements of young adult and old adult specimens of $A$ kodon montensis from EEB, separated by sex, and results of Student's t-test and its value of significance. Statistically significant values $(P<0.05)$ are indicated in bold.

\begin{tabular}{|c|c|c|c|c|c|c|c|c|}
\hline & \multicolumn{4}{|c|}{ Young adults } & \multicolumn{4}{|c|}{ Old adults } \\
\hline & Males & Females & $t$ & Sig. & Males & Females & $t$ & Sig. \\
\hline$\overline{\text { GSL }}$ & $28.03 \pm 1.03(8)$ & $27.70 \pm 0.87(4)$ & -0.561 & 0.587 & $30.03 \pm 0.77(10)$ & $29.44 \pm 0.59(11)$ & -1.968 & 0.064 \\
\hline CIL & $25.27 \pm 1.02(8)$ & $25.00 \pm 0.86(4)$ & -0.448 & 0.664 & $27.39 \pm 0.75(10)$ & $26.65 \pm 0.57(11)$ & -2.583 & 0.018 \\
\hline CZL & $18.53 \pm 0.77(8)$ & $18.34 \pm 0.66(4)$ & -0.429 & 0.677 & $19.99 \pm 0.53(10)$ & $19.59 \pm 0.44(11)$ & -1.924 & 0.069 \\
\hline LD & $7.39 \pm 0.42(8)$ & $7.28 \pm 0.24(4)$ & -0.475 & 0.645 & $8.13 \pm 0.35(10)$ & $7.87 \pm 0.32(11)$ & -1.787 & 0.09 \\
\hline LIF & $6.24 \pm 0.27(8)$ & $6.22 \pm 0.17(4)$ & -0.151 & 0.883 & $6.76 \pm 0.41(10)$ & $6.58 \pm 0.33(11)$ & -1.095 & 0.287 \\
\hline BIF & $2.41 \pm 0.18(8)$ & $2.34 \pm 0.18(4)$ & -0.643 & 0.535 & $2.56 \pm 0.17(10)$ & $2.38 \pm 0.14(11)$ & -2.594 & 0.018 \\
\hline LM & $4.17 \pm 0.11(8)$ & $4.18 \pm 0.19(4)$ & 0.073 & 0.943 & $4.21 \pm 0.16(10)$ & $4.19 \pm 0.13(11)$ & -0.243 & 0.810 \\
\hline BM1 & $1.15 \pm 0.05(8)$ & $1.17 \pm 0.06(4)$ & 0.532 & 0.606 & $1.20 \pm 0.06(10)$ & $1.19 \pm 0.05(11)$ & -0.338 & 0.739 \\
\hline ID & $1.35 \pm 0.06(8)$ & $1.34 \pm 0.05(4)$ & -0.298 & 0.772 & $1.48 \pm 0.04(10)$ & $1.40 \pm 0.04(11)$ & -4.091 & 0.001 \\
\hline IW & $1.44 \pm 0.11(8)$ & $1.48 \pm 0.08(4)$ & 0.646 & 0.533 & $1.60 \pm 0.05(10)$ & $1.55 \pm 0.10(11)$ & -1.364 & 0.189 \\
\hline LN & $10.99 \pm 0.55(8)$ & $10.76 \pm 0.57(4)$ & -0.681 & 0.512 & $12.04 \pm 0.55(10)$ & $11.69 \pm 0.45(11)$ & -1.598 & 0.126 \\
\hline BR & $3.74 \pm 0.20(8)$ & $3.56 \pm 0.15(4)$ & -1.588 & 0.143 & $3.92 \pm 0.17(10)$ & $3.77 \pm 0.15(11)$ & -2.048 & 0.055 \\
\hline $\mathbf{R L}$ & $10.75 \pm 0.51(8)$ & $10.61 \pm 0.29(4)$ & -0.498 & 0.629 & $11.82 \pm 0.51(10)$ & $11.55 \pm 11.82(11)$ & -1.458 & 0.161 \\
\hline ZB & $13.44 \pm 0.48(8)$ & $13.43 \pm 0.42(4)$ & -0.035 & 0.973 & $14.80 \pm 0.53(10)$ & $14.44 \pm 0.31(11)$ & -1.941 & 0.067 \\
\hline BZP & $2.41 \pm 0.11(8)$ & $2.38 \pm 0.07(4)$ & -0.571 & 0.581 & $2.61 \pm 0.18(10)$ & $2.54 \pm 0.15(11)$ & -0.963 & 0.347 \\
\hline LIB & $4.96 \pm 0.16(8)$ & $4.93 \pm 0.04(4)$ & -0.499 & 0.630 & $5.01 \pm 0.20(10)$ & $5.01 \pm 0.13(11)$ & -0.077 & 0.940 \\
\hline OFL & $8.87 \pm 0.28(8)$ & $8.89 \pm 0.22(4)$ & 0.102 & 0.921 & $9.42 \pm 0.25(10)$ & $9.24 \pm 0.14(11)$ & -2.001 & 0.060 \\
\hline PL & $11.28 \pm 0.47(8)$ & $11.21 \pm 0.48(4)$ & -0.225 & 0.827 & $12.13 \pm 0.46(10)$ & $11.77 \pm 0.37(11)$ & -2.008 & 0.059 \\
\hline LPB & $3.58 \pm 0.26(8)$ & $3.38 \pm 0.25(4)$ & -1.262 & 0.236 & $3.74 \pm 0.24(10)$ & $3.62 \pm 0.23(11)$ & -1.169 & 0.257 \\
\hline BBP & $2.83 \pm 0.09(8)$ & $2.77 \pm 0.11(4)$ & -0.946 & 0.367 & $3.09 \pm 0.11(10)$ & $3.07 \pm 0.13(11)$ & -0.417 & 0.681 \\
\hline HBC & $8.40 \pm 0.15(8)$ & $8.39 \pm 0.17(4)$ & -0.117 & 0.909 & $8.62 \pm 0.28(10)$ & $8.60 \pm 0.27(11)$ & -0.159 & 0.875 \\
\hline BBC & $12.49 \pm 0.36(8)$ & $12.34 \pm 0.08(4)$ & -1.049 & 0.324 & $12.65 \pm 0.31(10)$ & $12.44 \pm 0.29(11)$ & -1.545 & 0.139 \\
\hline IPB & $7.61 \pm 0.56(8)$ & $6.41 \pm 1.28(4)$ & -2.323 & 0.043 & $6.76 \pm 0.94(10)$ & $6.26 \pm 1.01(11)$ & -1.172 & 0.256 \\
\hline IPL & $1.69 \pm 0.29(8)$ & $1.57 \pm 0.34(4)$ & -0.629 & 0.543 & $1.60 \pm 0.43(10)$ & $1.88 \pm 0.47(11)$ & 1.428 & 0.169 \\
\hline$O C B$ & $6.27 \pm 0.09(8)$ & $6.27 \pm 0.18(4)$ & -0.117 & 0.909 & $6.50 \pm 0.15(10)$ & $6.43 \pm 0.13(11)$ & -1.199 & 0.245 \\
\hline
\end{tabular}

Table 11. Descriptive statistics (mean \pm standard deviation, range, and sample size) of body and cranio-dental measurements (mm), and weight ( $\mathrm{g}$ ), of adult specimens of Blarinomys breviceps from EEB, and descriptive statistics for the species obtained in Geise et al. (2008). *Values of males and females together.

\begin{tabular}{|c|c|c|c|c|}
\hline & \multicolumn{2}{|c|}{ EEB specimens } & \multicolumn{2}{|c|}{ Species values } \\
\hline & Males & Female & Males & Females \\
\hline HBL & 105.0 & 115.0 & $102.54 \pm 7.20(91.0-122.0) 23$ & $108.20 \pm 5.01(100.0-117.0) 11$ \\
\hline TL & 50.0 & 50.0 & $44.02 \pm 8.73(30.0-59.0) 22$ & $44.78 \pm 3.70(40.0-50.0) 9$ \\
\hline HF1 & 14.0 & 15.0 & 14.10 & $-16.0)^{*}$ \\
\hline HF2 & 16.0 & 18.0 & 17.00 & $-19.0)^{*}$ \\
\hline EL & 8.0 & 7.0 & - & - \\
\hline W & 36.5 & 33.5 & $33.31 \pm 9.31(12.5-49.0) 15$ & $35.89 \pm 3.44(29.5-39.5) 9$ \\
\hline GSL & $25.81 \pm 0.29(25.54-26.11) 3$ & 25.58 & - & - \\
\hline CIL & $22.78 \pm 0.45(22.48-23.30) 3$ & 22.61 & $23.50 \pm 0.74(21.56-24.63) 18$ & $24.28 \pm 0.84(23.18-25.72) 6$ \\
\hline CZL & $16.25 \pm 0.29(16.05-16.58) 3$ & 16.08 & - & - \\
\hline LD & $6.40 \pm 0.07(6.34-6.47) 3$ & 6.32 & $6.22 \pm 0.33(5.37-6.67) 20$ & $6.36 \pm 0.29(6.06-6.87) 7$ \\
\hline LIF & $5.36 \pm 0.16(5.23-5.54) 3$ & 4.92 & $4.83 \pm 0.31(4.36-5.37) 18$ & $4.90 \pm 0.25(4.55-5.38) 8$ \\
\hline BIF & $2.38 \pm 0.15(2.24-2.54) 3$ & 2.40 & $2.32 \pm 0.26(1.81-2.75) 20$ & $2.46 \pm 0.18(2.25-2.73) 8$ \\
\hline LM & $4.13 \pm 0.14(4.01-4.29) 3$ & 4.07 & $4.34 \pm 0.28(3.82-4.88) 20$ & $4.49 \pm 0.25(3.97-4.72) 8$ \\
\hline BM1 & $1.12 \pm 0.01(1.11-1.13) 3$ & 1.16 & $1.24 \pm 0.12(1.04-1.52) 20$ & $1.28 \pm 0.10(1.08-1.40) 8$ \\
\hline ID & $1.10 \pm 0.09(1.04-1.20) 3$ & 1.15 & - & - \\
\hline IW & $1.18 \pm 0.01(1.17-1.19) 2$ & 1.23 & - & - \\
\hline LN & $11.79 \pm 0.35(11.42-12.12) 3$ & 11.64 & - & - \\
\hline BR & $3.71 \pm 0.09(3.65-3.81) 3$ & 3.67 & - & - \\
\hline RL & $11.02 \pm 0.35(10.70-11.39) 3$ & 10.86 & $10.71 \pm 0.55(9.13-11.48) 19$ & $10.86 \pm 0.40(10.11-11.41) 8$ \\
\hline ZB & $12.99 \pm 0.08(12.90-13.06) 3$ & 13.11 & $13.47 \pm 0.68(12.33-14.43) 9$ & $14.65 \pm 0.06(14.60-14.72) 3$ \\
\hline BZP & $1.31 \pm 0.11(1.22-1.43) 3$ & 1.36 & $1.40 \pm 0.16(1.10-1.66) 18$ & $1.55 \pm 0.20(1.17-1.78) 7$ \\
\hline LIB & $6.82 \pm 0.07(6.75-6.89) 3$ & 6.81 & $6.63 \pm 0.31(6.03-7.15) 20$ & $6.75 \pm 0.35(5.91-7.01) 8$ \\
\hline OFL & $7.76 \pm 0.29(7.58-8.10) 3$ & 7.71 & $7.81 \pm 0.26(7.36-8.52) 20$ & $8.13 \pm 0.35(7.49-8.57) 7$ \\
\hline PL & $10.04 \pm 0.05(10.00-10.09) 3$ & 10.04 & - & - \\
\hline LPB & $3.95 \pm 0.10(3.83-4.01) 3$ & 4.32 & $4.19 \pm 0.22(3.64-4.48) 15$ & $4.14 \pm 0.26(3.83-4.63) 7$ \\
\hline BBP & $3.24 \pm 0.10(3.13-3.30) 3$ & 3.31 & - & - \\
\hline HBC & $8.69 \pm 0.20(8.46-8.81) 3$ & 8.28 & - & - \\
\hline BBC & $12.44 \pm 0.21(12.24-12.65) 3$ & 13.00 & - & - \\
\hline$O C B$ & $6.88 \pm 0.09(6.78-6.96) 3$ & 7.09 & $7.05 \pm 0.33(6.39-7.49) 18$ & $7.18 \pm 0.43(6.48-7.69) 6$ \\
\hline
\end{tabular}



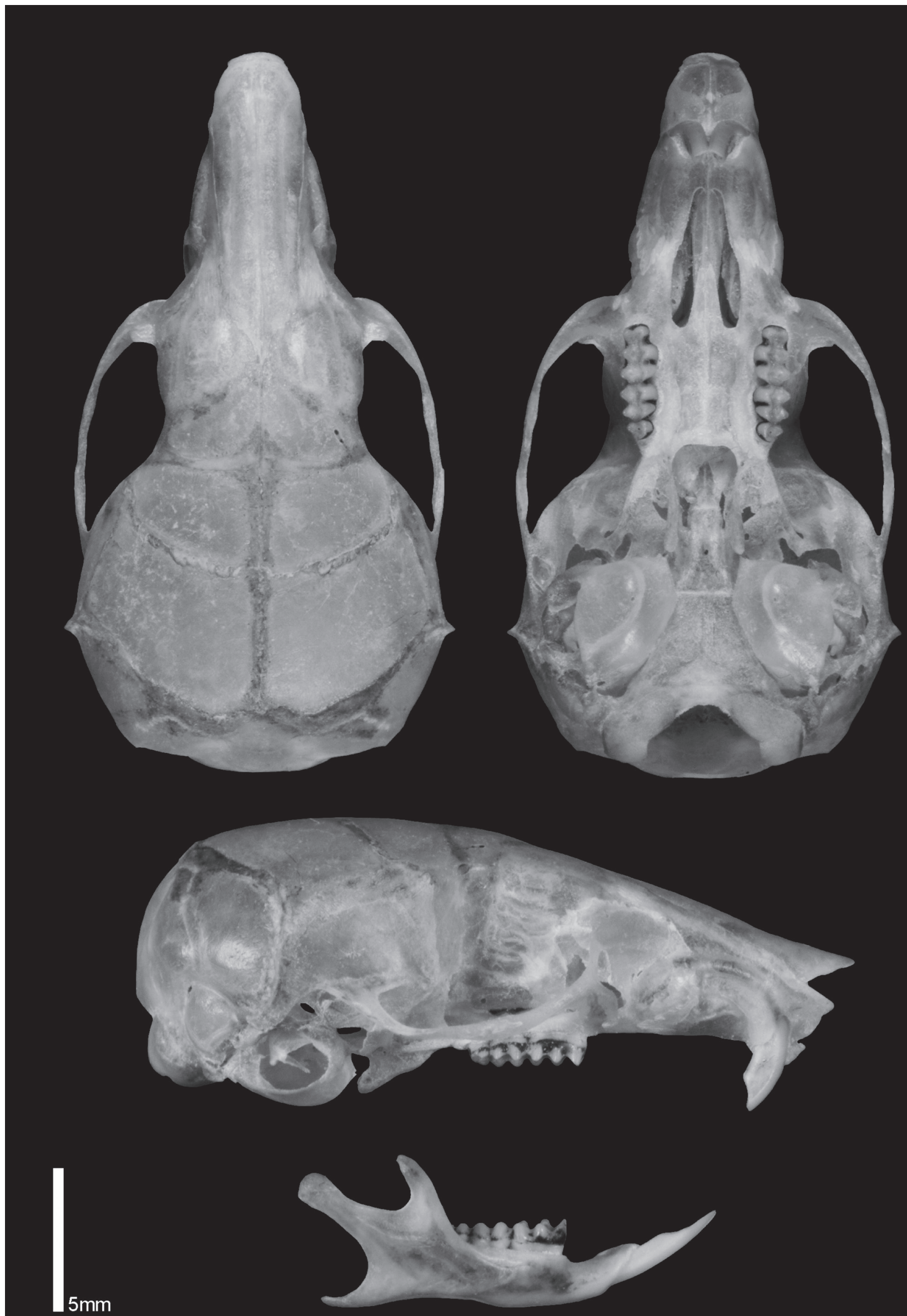

Figure 16. Skull and mandible of Blarinomys breviceps (EEB 776; female; GSL $=25.58 \mathrm{~mm}$ ). Skull on dorsal, ventral, and lateral view, and mandible on lateral view. 
and accessory oval foramen confluent (one specimen). Squamosal-alisphenoid groove present on braincase lateral view, in the squamosal and alisphenoid bones; sphenofrontal foramen present; posterior opening of alisphenoid canal large; stapedial foramen present and large in the ectotympanic bullae, on the petrosal-ectotympanic suture (stapedial and carotid circulatory pattern 1; Voss, 1988). Subsquamosal fenestra vestigial or absent. Postglenoid foramen present and large. Mastoid with conspicuous fenestra. Incisive foramina teardrop on shape, with anterior portion notably narrow; posterior margin of incisive foramina reaching the anterior face of $\mathrm{M} 1$ (two specimen) or surpassing the anterior face of M1 (two specimen). Palate short and wide, with the central region deeper; posterolateral palatal pits present as one single opening on the right side of posterior palate (two specimens) or present as one single opening on the left side and two openings on the right side (two specimens). Mesopterygoid fossa reaching the posterior face of M3 (three specimens) or surpassing the posterior face of M3 (one specimen); sphenopalatine vacuities present but reduced as narrow openings close to basisphenoid-presphenoid suture. Parapterygoid plate narrower than the middle portion of mesopterygoid fossae (three specimens) or with similar width to the middle portion of mesopterygoid fossae (one specimen). Auditory bullae large, periotic bone mostly masked in ventral view. Mandible elongated, with low ramus (Fig. 16). Superior and posterior notches very deep. Capsular process of lower incisor alveolus absent. Superior and inferior masseteric ridges very subtle and with anterior portion conjoined as single crest. Upper incisors opisthodont. Maxillary toothrows parallels or slightly anteriorly divergent. M1 with anterocone not divided into labial and lingual conules (anteromedian flexus absent), but with the central region of anterior surface slightly deeper; anteroloph and anteroflexus absent; protostyle absent; mesoloph absent; enterostyle absent. $\mathrm{m} 1$ with anteroconid not divided, anteromedian flexid absent; anterolophid present but very reduced (vestigial); protostylid present but reduced; mesolophid absent; ectolophid and ectostylid absent.

Remarks: Comparison of EEB specimens with the morphological descriptions of $B$. breviceps by Geise et al. (2008) reveals that most characteristics found in EEB specimens are within the variation proposed for the species. However, we observed some cranial structures in EEB specimens that, according to Geise et al. (2008), are not present in $B$. breviceps: the posterolateral palatal pits and the sphenopalatine vacuities. Additionally, the EEB specimens did not present a developed capsular process of the lower incisor alveolus, contrasting with what Geise et al. (2008) attributed to the species. Even though the latter changes with age (it can be absent in juveniles and present in adults), all EEB specimens analyzed by us were adults, in which this structure, when present, should be well developed. All biometric characters (body and cranio-dental), obtained from EEB specimens, are within the values observed for the species (Geise et al., 2008) (see Table 11).
Field observations: Five specimens of $B$. breviceps were collected at EEB, four males and one female, which were captured with pitfall traps in December, 2003, and April, 2004. Two specimens captured in December 2003, one male and one female, were reproductively active: the male had scrotal testes and the female had perforated vagina and visible mammary glands. The other specimens, three males, captured in April, 2004, did not show signs of reproductive activity.

\section{Genus Brucepattersonius}

The genus Brucepattersonius was described by Hershkovitz (1998), who included in its contents B. iheringi (previously Oxymycterus iheringi Thomas) and four new species: $B$. soricinus (type species of the genus), $B$. igniventris, $B$. griserufescens and $B$. albinasus. Besides the four new names, Hershkovitz included in the genus one unnamed species from Misiones, Argentina. Two years later, Mares \& Braun (2000) described three additional species based on three specimens collected in Misiones: B. paradisus, B. misionensis and B. guarani. After morphological comparison with holotypes and molecular analyses, Vilela et al. (2006) found no substantial support to hold the recognition of $B$. griserufescens and $B$. albinasus as distinct taxa, suggesting the synonymy of $B$. albinasus under B. griserufescens. As a result, Brucepattersonius currently includes seven species (Vilela et al., 2015). However, the taxonomy of the genus is far from stable. For instance, the validity of the three species described by Mares \& Braun (2000), based on one specimen each and from very close localities in Misiones, has been questioned (e.g., Pereira et al., 2005; Jung, 2009; Cirignoli et al., 2011; Vilela et al., 2015), as well the legitimacy of $B$. soricinus and B. igniventris as distinct taxa (e.g., Vilela et al., 2015; Dias, 2016).

Fifteen specimens of Brucepattersonius were identified at EEB sample, and for six of them we sequenced the CYTB gene. To confirm their identities and phylogenetic relationships, we generated additional sequences for two specimens from Rio Grande do Sul (MCNU 2820 from Dois Lajeados municipality) and Santa Catarina (MCNU 2795 from Ouro municipality) states, representing $B$. iheringi. Sequences from B. griserufescens were kindly provided by D. Dias and Y.L.R. Leite for two specimens from the Parque Nacional do Caparaó, Espírito Santo State (UFES 1128 and 1161). J.L. Patton generously make available sequences for $B$. soricinus (LG 123 from Itatiaia, Rio de Janeiro State) and from Brucepattersonius sp. (MF 12 from Passa Quatro, Minas Gerais State, and LG 108 from Itatiaia, Rio de Janeiro State). Finally, we obtained three GenBank sequences, two of those identified as B. soricinus (MN 78974 from Natividade da Serra and MVZ 183036 from Boracéia, São Paulo State) and one associated to B. igniventris (MVZ 183250 from Intervales, São Paulo State).

Our phylogenetic inferences based on $\mathrm{ML}$ and $\mathrm{BI}$ showed identical topologies (Fig. 17). Notwithstanding that monophyly tests were far from our intention here, 
Brucepattersonius was recovered as a natural group with strong support on $\mathrm{BI}$, but only moderate on ML. The 16 Brucepattersonius specimens used as terminals composed four strongly supported clades, with mean sequence divergences ranging from $5.75 \%$ to $8.44 \%$ among them (Table 12). Specimens from EEB fell into two clades: one of them, includes specimens from Itatiaia, Rio de Janeiro State, and Passa Quatro, Minas Gerais State, along with EEB specimens. This clade is sister to a clade composed by specimens from Parque Nacional do Caparaó, Espírito Santo State, type locality of $B$. griserufescens. The remaining specimens from EEB clustered with samples from throughout São Paulo State and south of Rio de Janeiro
State, including specimens associated to $B$. soricinus and $B$. igniventris. We assigned this clade to $B$. soricinus following Vilela et al. (2015) and unpublished data (Abreu-

Table 12. CYTB mean divergence ( $p$-distance) between and within (in bold) species of Brucepattersonius.

\begin{tabular}{lcccc}
\hline & $\begin{array}{c}\text { Brucepattersonius } \\
\text { n. sp. }\end{array}$ & B. griserufescens & B. soricinus & B. iheringi \\
\hline Brucepattersonius n. sp. & $\mathbf{1 . 1 8 \%}$ & & & \\
$\begin{array}{l}\text { B. griserufescens } \\
\text { B. soricinus }\end{array}$ & $5.80 \%$ & $\mathbf{0 . 3 7 \%}$ & & \\
B. iheringi & $8.24 \%$ & $7.40 \%$ & $\mathbf{0 . 7 1 \%}$ & \\
\hline
\end{tabular}

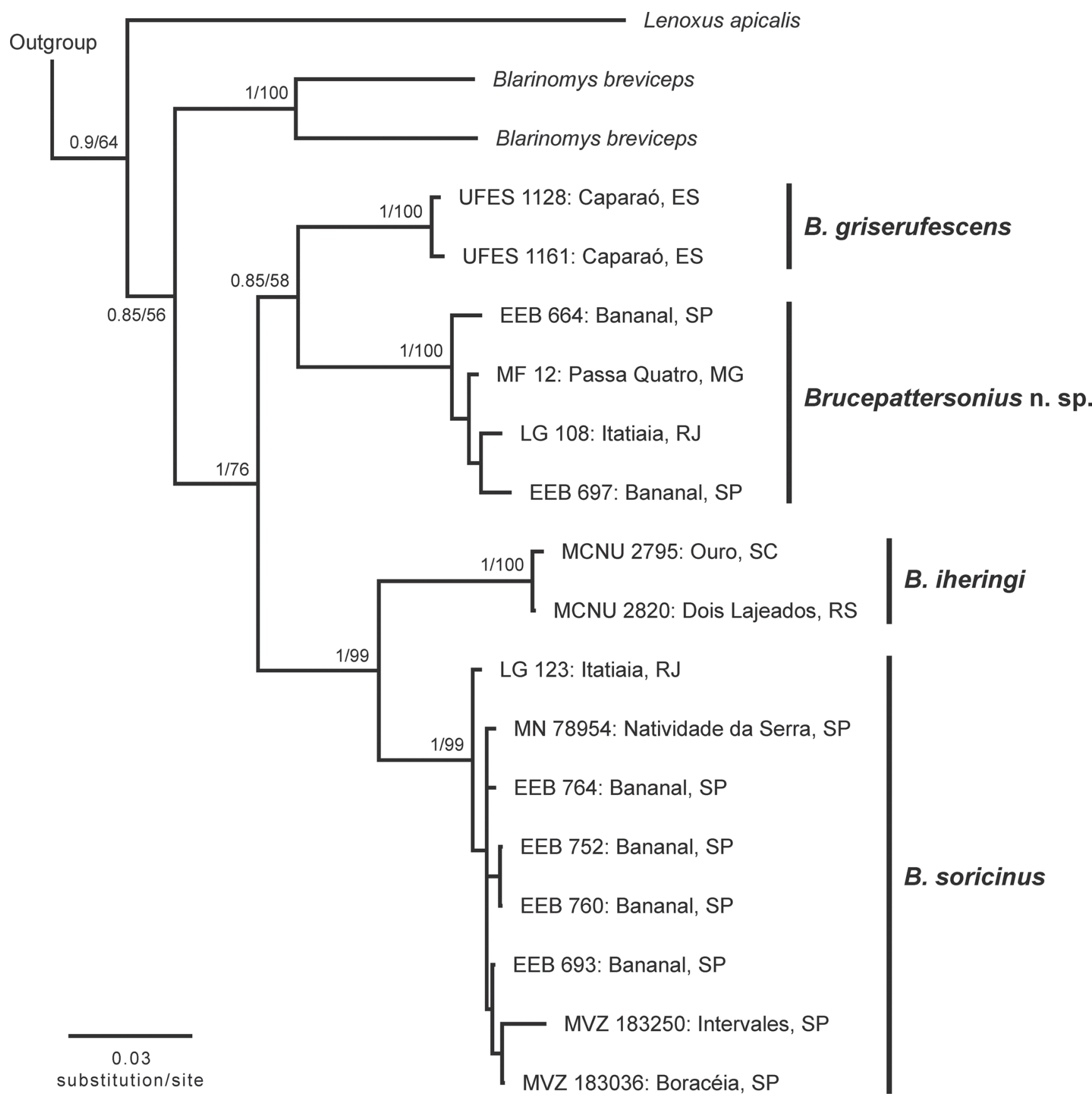

Figure 17. Bayesian Inference topology based on CYTB sequences from 22 specimens of Brucepattersonius, plus one Lenoxus apicalis (U03541) and two Blarinomys breviceps (AF108668 and AY275112). We included Scapteromys tumidus (AY275133), Kunsia tomentosus (AY275121) and Bibimys labiosus (DQ444329) as outgroups. The nucleotide substitution model suggested by the Akaike Information Criterion (AIC) was GTR + I + G4. Support values (close to the nodes) are BI posterior probabilities and bootstrap replicates (obtained from ML analysis). 
Júnior et al., in prep.), which suggest that $B$. igniventris may not be distinct from $B$. soricinus, and thus possibly representing a single entity. This decision was strengthened by our morphological analyses (see discussion on the remarks of $B$. soricinus as follow). The sister clade of the former is composed by specimens from Rio Grande do Sul and Santa Catarina states. Along the geographic range of the samples in this clade is the type locality of B. iheringi.

Therefore, we hypothesized that the clade composed by two specimens from EEB plus specimens from Itatiaia and Passa Quatro represent an unnamed lineage in the genus Brucepattersonius. To better evaluate the taxonomic status of this lineage, we conducted morphometric and morphologic analyses comparing specimens from localities sampled in this clade with specimens from the sister-clade (B. griserufescens) and with sympatric specimens of $B$. soricinus.

We performed a multivariate analysis (PCA) using 21 cranio-dental variables. The results corroborate the molecular approach and showed these three lineages morphometric segregated (Fig. 18). The first two components accounted for $35 \%$ of the variation, and variables representing skull length (e.g., GSL, CIL, LD and RL) contributed largely on the first component (Table 13). In the second component, along with two variables also representing skull length (CZL and OFL), dimensions related to the zygomatic plate (BZP) and teeth (ID) were determinant to explain the observed variation of $19.32 \%$. One specimen of $B$. griserufescens overlapped with specimens of Brucepattersonius n. sp. - an old female which is the only old adult, AC5, in the sample. Also, one specimen of $B$. soricinus overlapped with Brucepattersonius n. sp., in this case, a young-adult male AC2. Specimens from the new species were split in two clusters along the PC 1, possibly reflecting the ontogenetic variation of the sample, as the oldest specimens were positioned towards negative values and the youngest specimens towards positive values of the PC1.

We also generated standard boxplots using absolute values for the cranio-dental variables to compare the samples above mentioned. We found three variables distinguishing all samples (Fig. 19). Two of those were very conserved dimensions, which rarely vary across the ontogenetic development (LM and BZP), being reliable as diagnostic features for these species. We observed a size variation in these two variables reflecting the latitudinal gradient, as the northern species (B. griserufescens) exhibited the narrowest zygomatic plate (BZP), which increased in size in the southern species, Brucepattersonius n. sp. and $B$. soricinus, respectively. On the other hand, the length of molar (LM) decreased from north (B. griserufescens has the longest molar series) to south ( $B$. soricinus has the shorter molar series). The LPB did not exhibit such north-south pattern of variation, although $B$. griserufescens presented the longest palatal bridge.

Some variables were powerful to distinguish Brucepattersonius n. sp. from B. griserufescens (Fig. 20). For instance, $B$. griserufescens showed thinner incisors (ID) and longer diastema and palate (LD and PL), when compared to the new species. Some other variables segregated Brucepattersonius n. sp. from B. soricinus (Fig. 21), the latter with broader rostrum in comparison with Brucepattersonius n. sp., as demonstrated by values of BIF and BR.

Besides genetic (5.8\% on CYTB) and morphometric divergences, specimens of Brucepattersonius n. sp. differed from specimens of $B$. griserufescens on morphological qualitative traits. In the external morphology (Fig. 22), B. griserufescens exhibited the dorsal pelage

Table 13. Scores of cranio-dental morphometric variables in the first two principal components from a PCA of Brucepattersonius samples. Variables that loaded higher on each component are indicated in bold.

\begin{tabular}{lcc}
\hline \multicolumn{1}{c}{ Variables } & PC1 & PC2 \\
\hline GSL & $\mathbf{1 2 . 2 7 0}$ & 0.029 \\
CIL & 11.815 & 0.791 \\
CZL & 1.250 & 16.836 \\
LD & 11.349 & 0.194 \\
LIF & 3.527 & 3.933 \\
BIF & 0.038 & 7.628 \\
LM & 2.006 & 8.038 \\
BM1 & 2.681 & 0.003 \\
ID & 0.269 & 12.128 \\
LN & 3.969 & 2.870 \\
BR & 0.263 & 0.583 \\
RL & $\mathbf{1 1 . 1 5 5}$ & 0.510 \\
ZB & 6.523 & 3.010 \\
BZP & 0.057 & $\mathbf{1 5 . 1 9 4}$ \\
LIB & 6.287 & 2.766 \\
OFL & 0.030 & $\mathbf{1 5 . 6 3 8}$ \\
PL & 10.681 & 0.817 \\
LPB & 4.011 & 2.445 \\
BBP & 3.516 & 3.761 \\
BBC & 5.453 & 1.320 \\
OCB & 2.852 & 1.508 \\
\hline
\end{tabular}

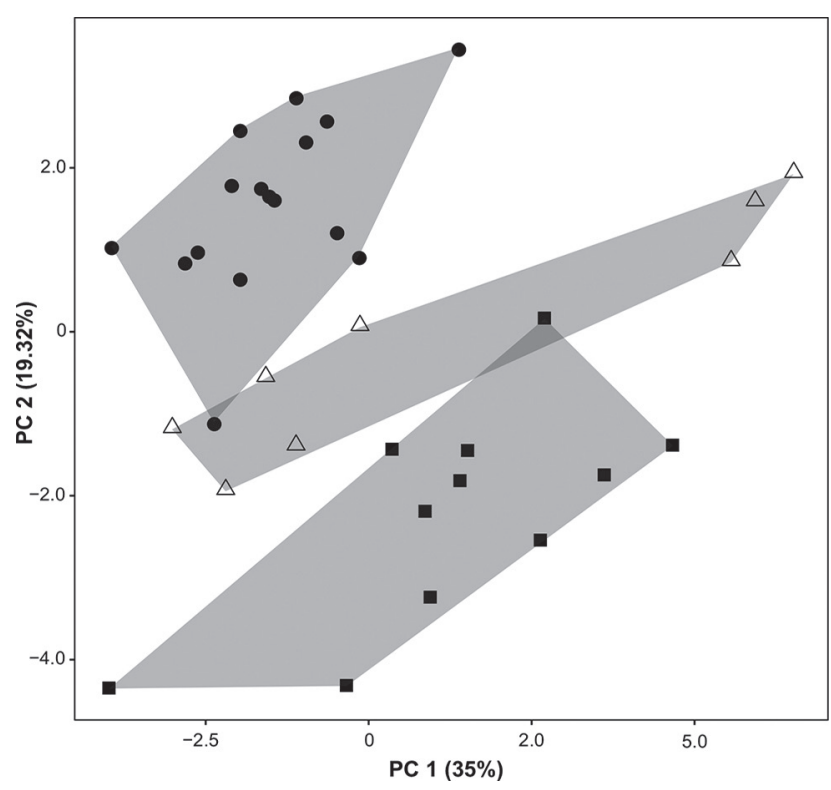

Figure 18. Scatter plot graph showing the projections of individual scores of Brucepattersonius griserufescens (black dots), Brucepattersonius n. sp. (open triangles) and $B$. soricinus (black squares) from a PCA analysis. The polygons in gray show the external limits of each sample. 
denser than Brucepattersonius n. sp. The ventral pelage of $B$. griserufescens showed a larger portion of white hairs, specially in the gular region, configuring an overall whitish pattern more evident, while in the new species the overall pattern tends to grayish-buff. The tail is usually shorter than head and body length in the new species (averaging 98\% of head in body length in eight specimens), while in $B$. griserufescens the tail is usually longer than head and body (averaging 105\% of head in body length in seven specimens). The tail dorsal hairs are about 1 scale long in $B$. griserufescens, while in Brucepattersonius n. sp. they reach two scales long;
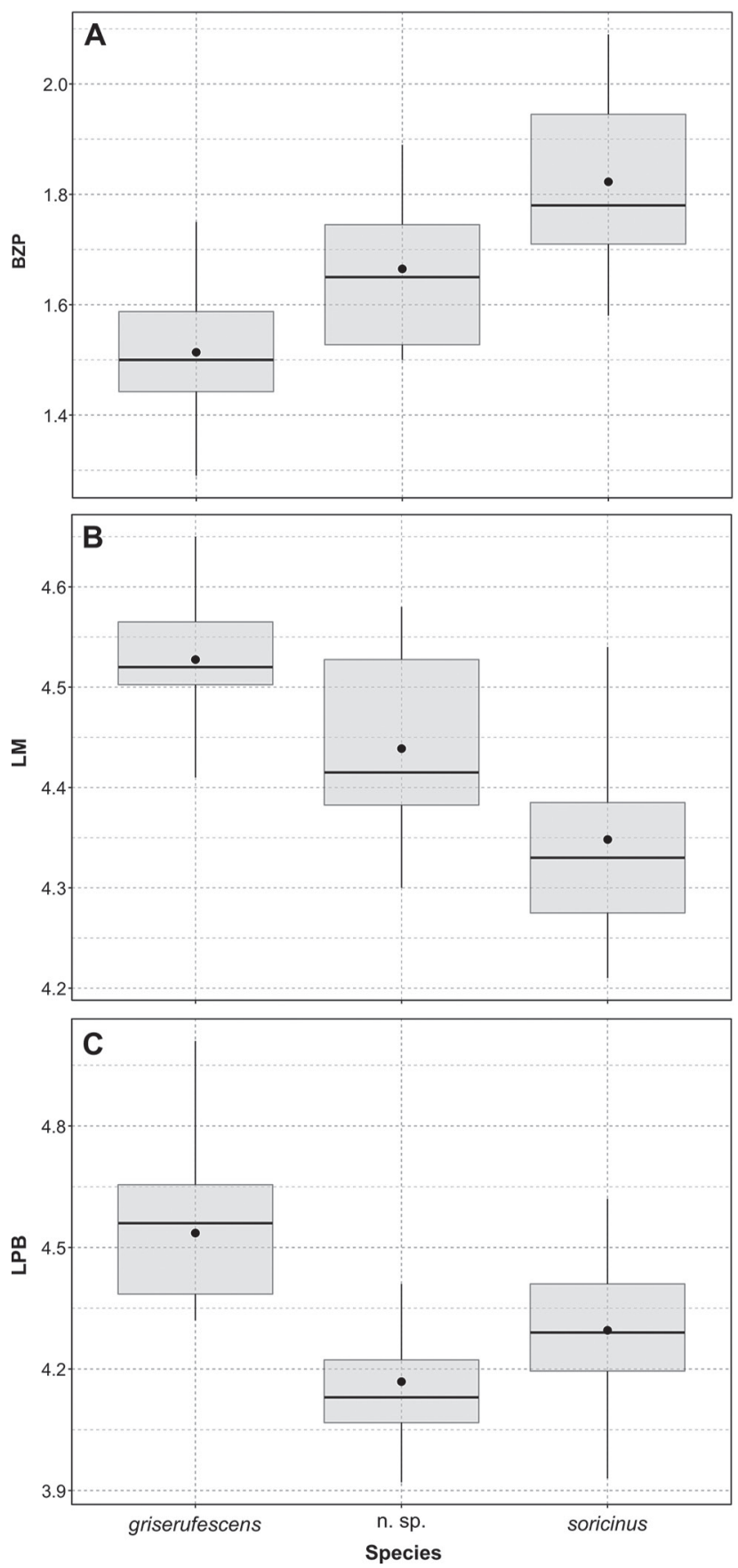

Figure 19. Boxplots showing the median (black line), mean (black dot), first and third quartiles, and minimum and maximum values for BZP (A), LM (B) and LPB (C), comparing Brucepattersonius n. sp. with B. griserufescens from Caparaó and B. soricinus from EEB. hairs on the ventral surface of the tail are predominantly whitish in B. griserufescens, while the majority of tail ventral hairs are brownish in Brucepattersonius n. sp. The claws of pes are longer in B. griserufescens (about $4 \mathrm{~mm}$ long), while in Brucepattersonius n. sp. they average $3 \mathrm{~mm}$ long. In the skull morphology, the nasals of Brucepattersonius n. sp. extend posteriorly beyond the maxillary-frontal-lacrimal suture, while in B. griserufescens the posterior margin of nasals are collinear or anterior to the maxillary-frontal-lacrimal suture. The incisive foramina of Brucepattersonius n. sp. has the central portion broader than the anterior (very narrow) and the
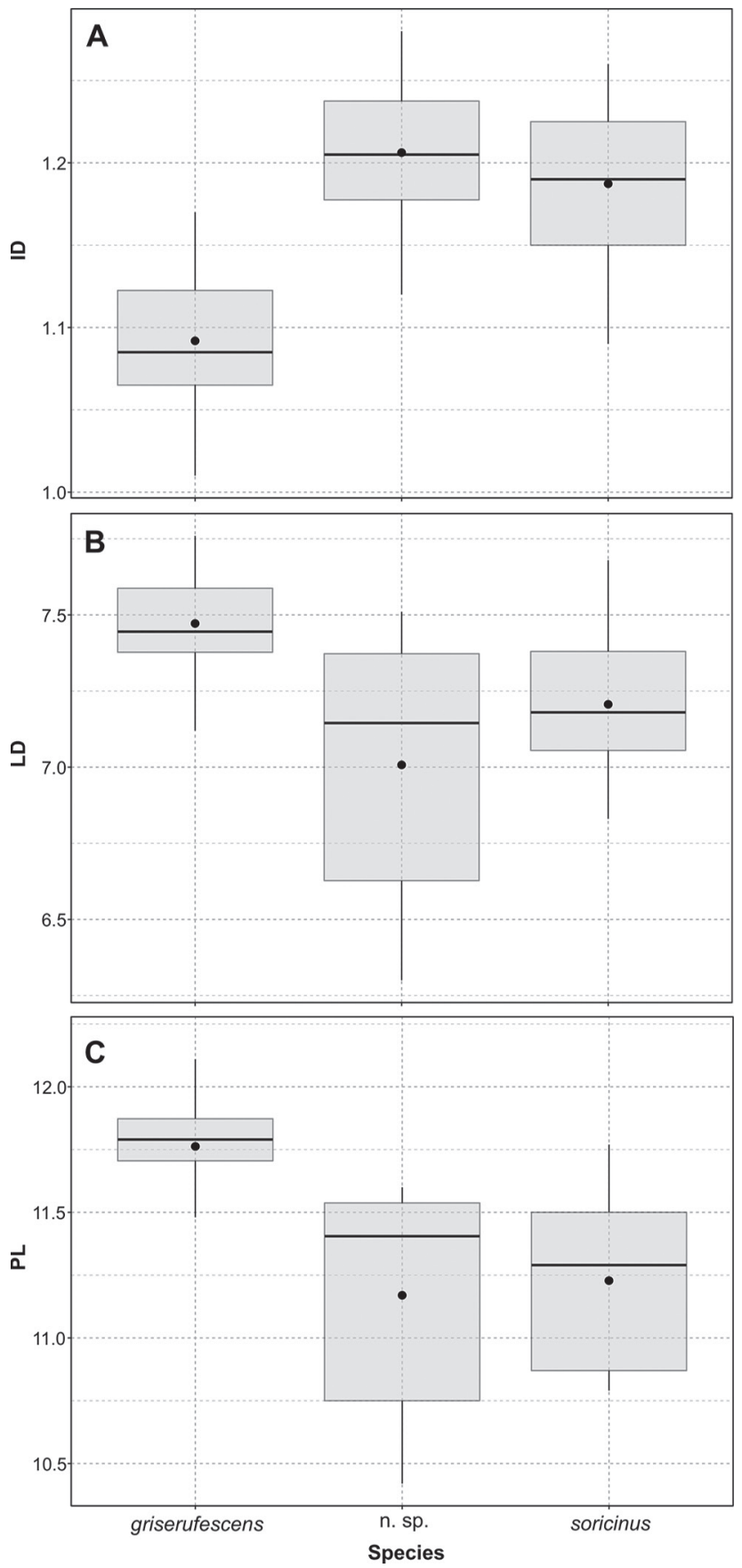

Figure 20. Boxplots showing the median (black line), mean (black dot), first and third quartiles, and minimum and maximum values for ID (A), LD (B) and $\mathrm{PL}(\mathrm{C})$, comparing Brucepattersonius n. sp. with B. griserufescens from Caparaó and B. soricinus from EEB. 
posterior portions, while in B. griserufescens the central portion presents similar breadth than the posterior portion; moreover, the posterior margin of the incisive foramina penetrates the molar series reaching the protoflexus in Brucepattersonius n. sp., while in B. griserufescens the incisive foramina is shorter, ending collinear to anterior surfaces of the first molars (Fig. 23). The nasal and premaxillae form a rostral tube, that is strongly built and well projected anteriorly in B. griserufescens, and more delicate and shorter in the new species; the gnathic process of $B$. griserufescens is more robust than in the new species (Fig. 24). In ventral view, the premaxillae are
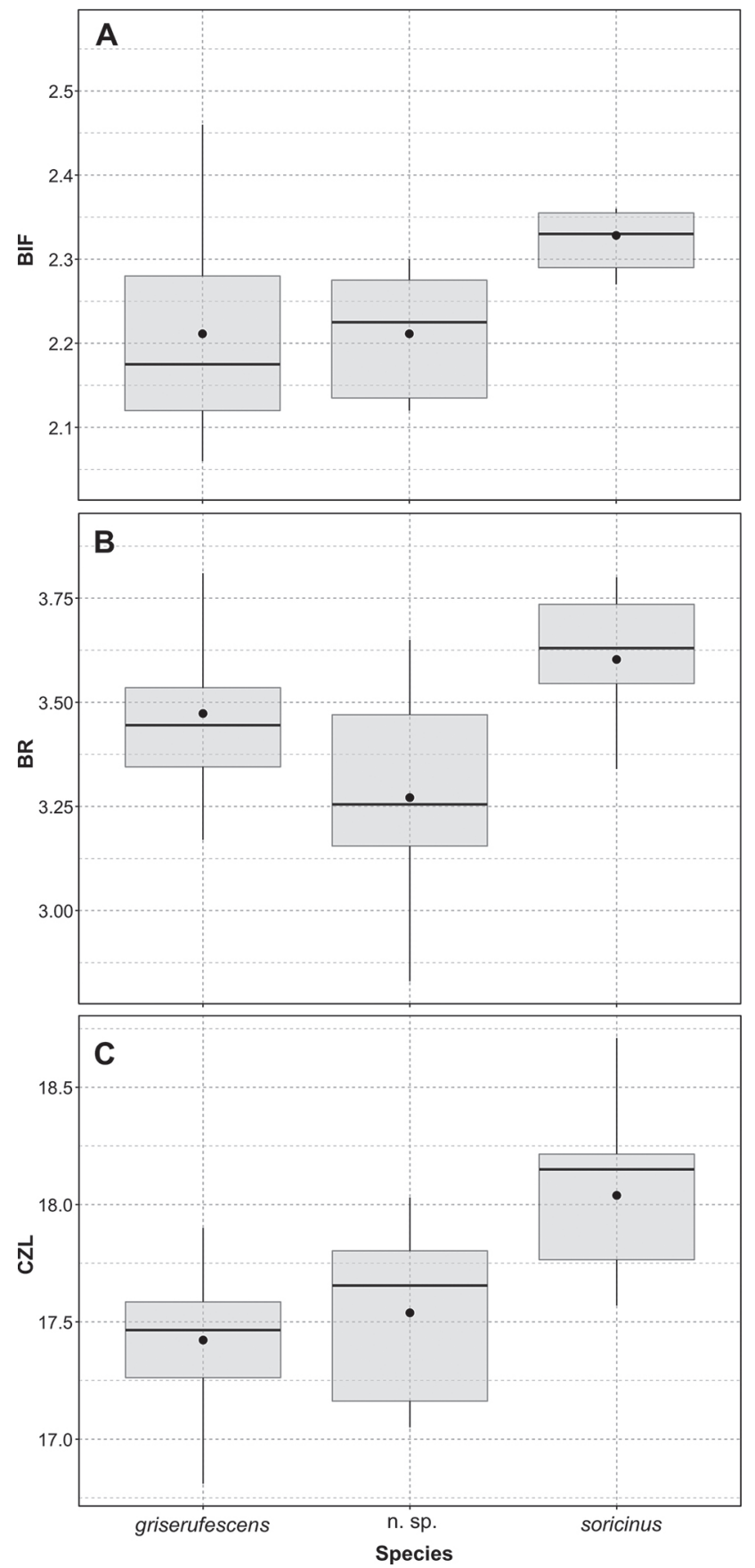

Figure 21. Boxplots showing the median (black line), mean (black dot), first and third quartiles, and minimum and maximum values for BIF (A), BR (B) and $\mathrm{CZL}(\mathrm{C})$, comparing Brucepattersonius $\mathrm{n}$. sp. with B. griserufescens from Caparaó and B. soricinus from EEB. joined medially, forming a closed ring and configuring a small nasal aperture in $B$. griserufescens, while in the new species the premaxillaries are distantly positioned, configuring a wider nasal aperture (Fig. 23).

Comparing specimens of Brucepattersonius $\mathrm{n}$. sp. with sympatric specimens of $B$. soricinus from EEB, a consistent set of morphological differences were observed. In the external morphology (Fig. 22), the dark brown dorsal color of Brucepattersonius n. sp. differed from the copperish-brown coloration of $B$. soricinus. The limit between the lateral and ventral colors is conspicuous (though slightly) in Brucepattersonius n. sp., with the venter more grayish, and this differs from the condition observed in $B$. soricinus, in which such contrast is not present, in part due to the orange coloration of the venter that is intermixed with the lateral coloration. The tail is unicolor dorsoventrally, dark-brown in Brucepattersonius n. sp., but predominantly bicolor in B. soricinus, dark-brown dorsally and white ventrally. In the cranial morphology, the rostrum of Brucepattersonius $\mathrm{n}$. $\mathrm{sp}$. is narrow, with the premaxillary weakly flared laterally on anterior portion (Fig. 23), while the rostrum of $B$. soricinus is conspicuously broader (see Fig. 27). The zygomatic plate of $B$. soricinus is more expanded laterally, forming a wide zygomatic notch, with posterior margin approximately close to alveolus of $\mathrm{M} 1$, whereas the zygomatic plate in Brucepattersonius n. sp. is less expanded laterally, configuring a narrow zygomatic notch, and with posterior margin situated posteriorly to alveolus of M1. The dorsal root of the zygomatic process of the maxilla is also more robust (wider) in B. soricinus, which also has the lacrimal more pronounced laterally, differing from Brucepattersonius n. sp. In Brucepattersonius n. sp., incisive foramina with convex lateral margins are more frequent, a feature not evident in $B$. soricinus, even though this structure is very variable in the second species. The hamular process of the squamosal is usually wider in B. soricinus, configuring smaller and narrower subsquamosal fenestra and postglenoid foramen, and narrower in Brucepattersonius n. sp, resulting in large and ample fenestra and foramen. The coronoid process of the mandible is more rounded and curved in B. soricinus, whereas in Brucepattersonius n. sp. it is more acute and less curved.

Based on the congruence of evidences obtained from distinct sources (e.g., genetic, morphometric, morphologic), we present the formal description of a new species for the genus Brucepattersonius.

\section{Brucepattersonius nebulosus new species E.F. Abreu-Júnior, J.F. Vilela, A.U. Christoff, V.H. Valiati \& A.R. Percequillo}

\section{Nebulous Brucie}

Holotype: MZUSP 35847, a young-adult male, consisting of skin, skull, carcasses, tissue preserved in ethanol and CYTB gene sequence. The holotype was collected with pitfall trap during the first field expedition to EEB in 14 December 2003, under the field number EEB 697. 

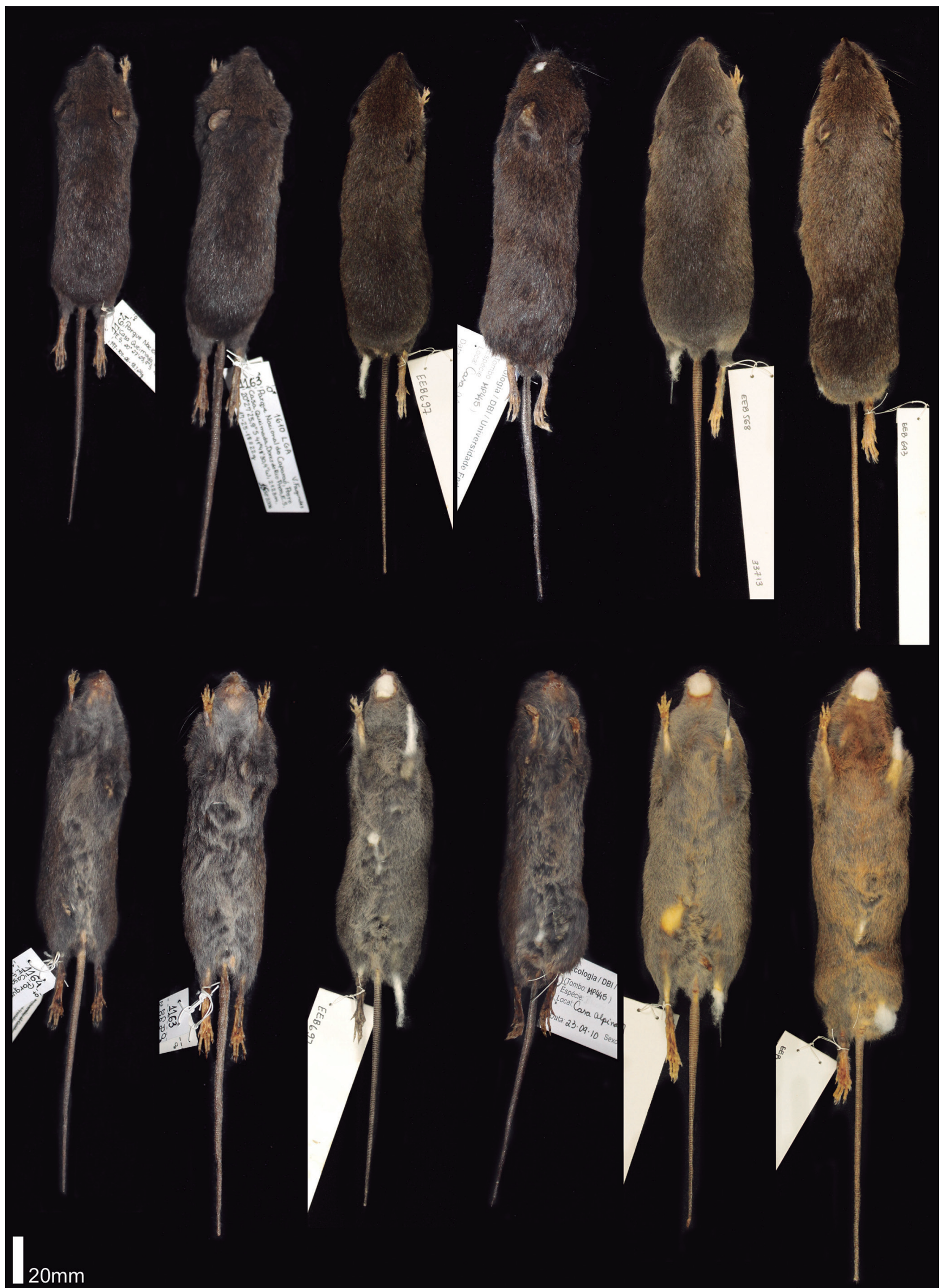

Figure 22. Skins (dorsal and ventral views) of (from left to right) Brucepattersonius griserufescens (UFES 1164 and 1163), Brucepattersonius n. Sp. (MZUSP 35847 and MP 445) and B. soricinus (MZUSP 33713 and 33758). 
Paratypes: We assigned all other specimens of $B$. nebulosus collected at EEB as paratypes: MZUSP 33714 (original number EEB 664), a female collected in 12 December 2003, consisting of skin (tail damaged), skull, carcasses and tissue preserved in ethanol (CYTB sequence available); MZUSP 35848 (original number EEB 698), a female collected in 14 December 2003, consisting of skin, skull, carcasses and tissue preserved in ethanol; MZUSP 35846 (original number EBM 023), a male collected in 14 April 2004 , consisting of skull and body in fluid.

Other specimens examined and sequence data: MINAS GERAIS: Itamonte: Casa Alpina: MP 312, MP 445, MP 481 (sequence data provided in Dias, 2016), MP 491,
MP 531; Passa Quatro: Fazenda do Itaguaré, 16 km SW: UFMG 1891 (MF 12; sequence data generated by James $L$. Patton). RIO DE JANEIRO: Itatiaia: Brejo da Lapa: LG 108 (sequence data generated by James L. Patton); Paraty: Parque Nacional da Serra da Bocaina: BOCA 667 (MN uncatalogued; sequence data provided in Dias, 2016), BOCA 795 (MN uncatalogued, sequence data provided in Dias, 2016); Teresópolis: Parque Nacional da Serra dos Órgãos: RF 77 (sequence data provided in Dias, 2016).

Type locality: Brazil, São Paulo State, Bananal municipality, Estação Ecológica de Bananal. The holotype was collected at Trilha da Pedra Vermelha $\left(22^{\circ} 47^{\prime} 53^{\prime \prime} \mathrm{S}\right.$, $44^{\circ} 21^{\prime} 35^{\prime \prime} \mathrm{W}$ ) around 1,200 m above sea level. The Estação

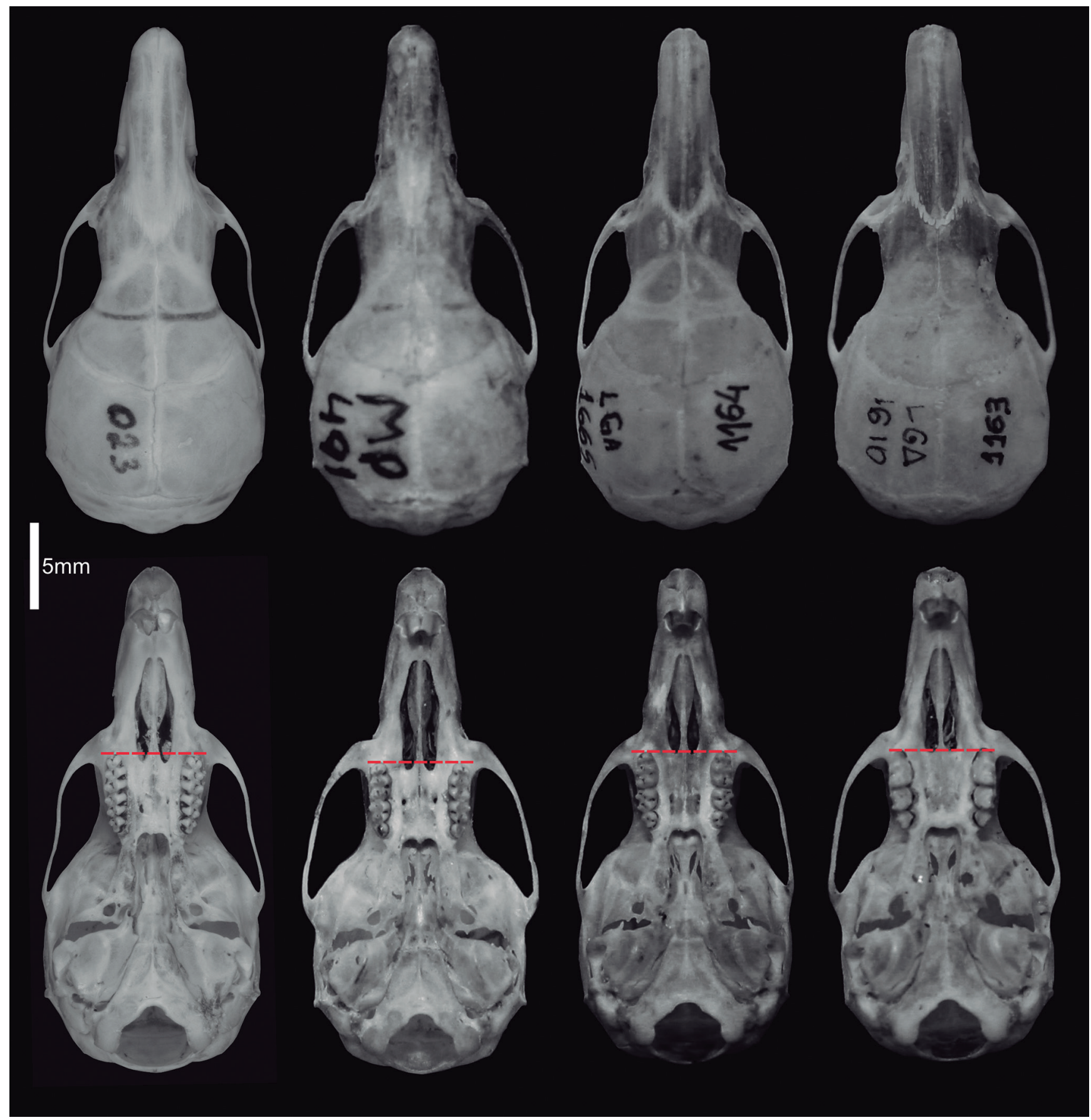

Figure 23. Skull (dorsal and ventral views) of (from left to right) Brucepattersonius n. sp. (MZUSP 35846 [EBM 023] and MP 491) and B. griserufescens (UFES 1164 and 1163). The red line highlights the length of the incisive foramina with posterior margin penetrating or between the molar series. 
Ecológica de Bananal is located in the Serra da Bocaina mountain range on the east side of the Rio Paraíba do Sul.

Etymology: This new species was named "nebulosus" in allusion to its occurrence in "misty" montane forests along the highlands of the southeastern Atlantic Forest, and also to its taxonomic status that remained overlooked and unclear for decades.

Geographic distribution: Brucepattersonius nebulosus is currently known from the slopes of the mountain ranges along both banks of the Rio Paraíba do Sul valley in the southeastern Atlantic Forest (Fig. 25). Two localities are from the Serra da Bocaina (EEB and Parque Nacional da Serra da Bocaina) and one locality from the Serra dos
Órgãos (Parque Nacional da Serra dos Órgãos), both ranges part of the Serra do Mar system, and three localities from the Serra da Mantiqueira (Parque Nacional do Itatiaia, Itamonte and Passa Quatro).

Morphological diagnosis: This species is diagnosed from other species of the genus Brucepattersonius by a unique combination of morphological traits, as dark brown dorsal pelage, ventral pelage grayish-buff slightly whitish, tail usually shorter than head and body length and uniformly dark brown, skull with narrow rostrum, long incisive foramina (penetrating the molar series and reaching the protoflexus) and short palatal bridge.

Morphological description: Medium size (see body measurements on Table 14). Dorsal pelage dense and

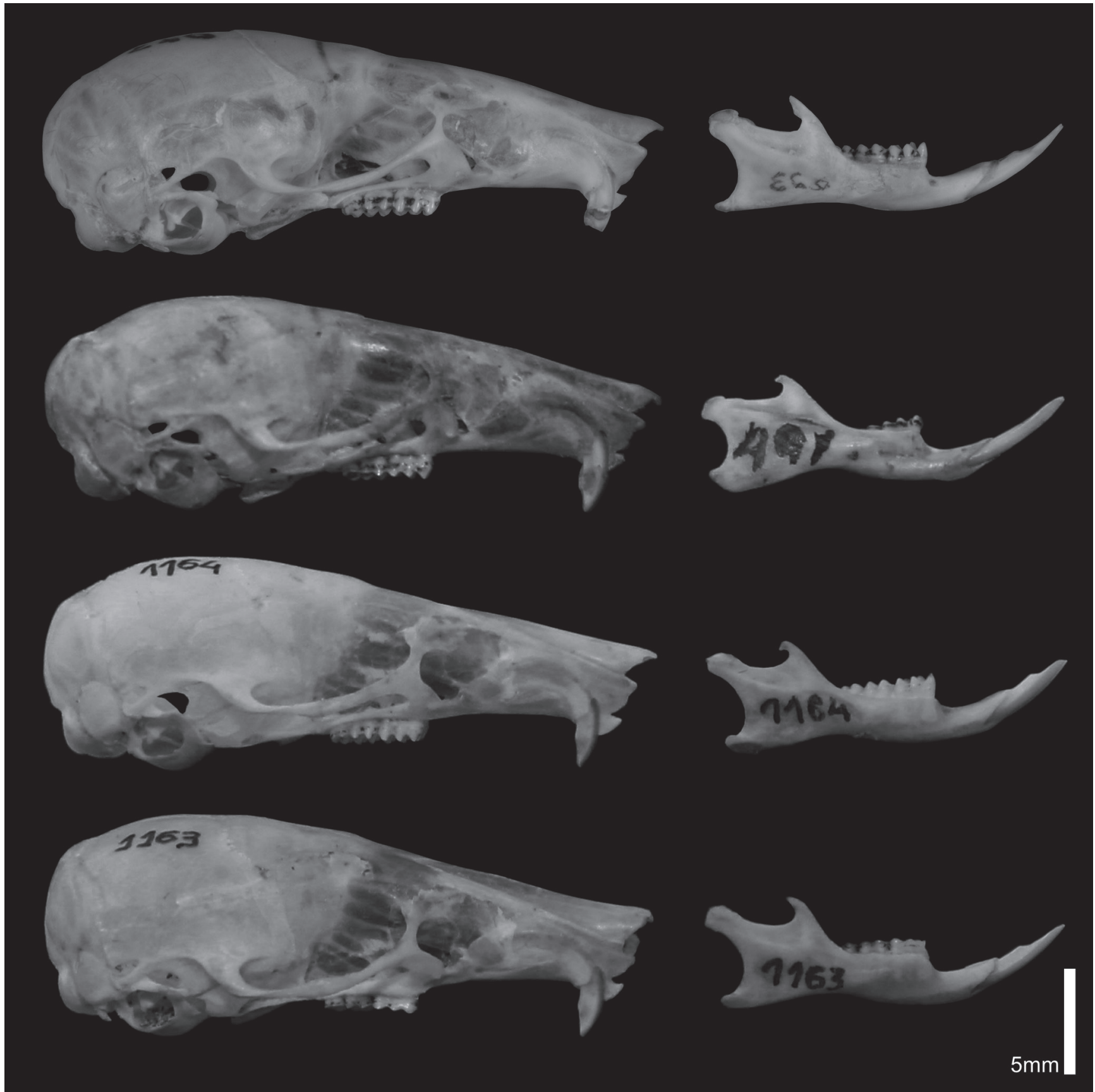

Figure 24. Skull (lateral view) and mandible of (from top to down) Brucepattersonius n. sp. (MZUSP 35846 and MP 491) and B. griserufescens (UFES 1164 and 1163 ). 
Table 14. Descriptive statistics (mean \pm standard deviation, range, and sample size) of body and cranio-dental measurements ( $\mathrm{mm})$, and weight $(\mathrm{g})$, of Brucepattersonius nebulosus n. sp., B. griserufescens and B. soricinus.

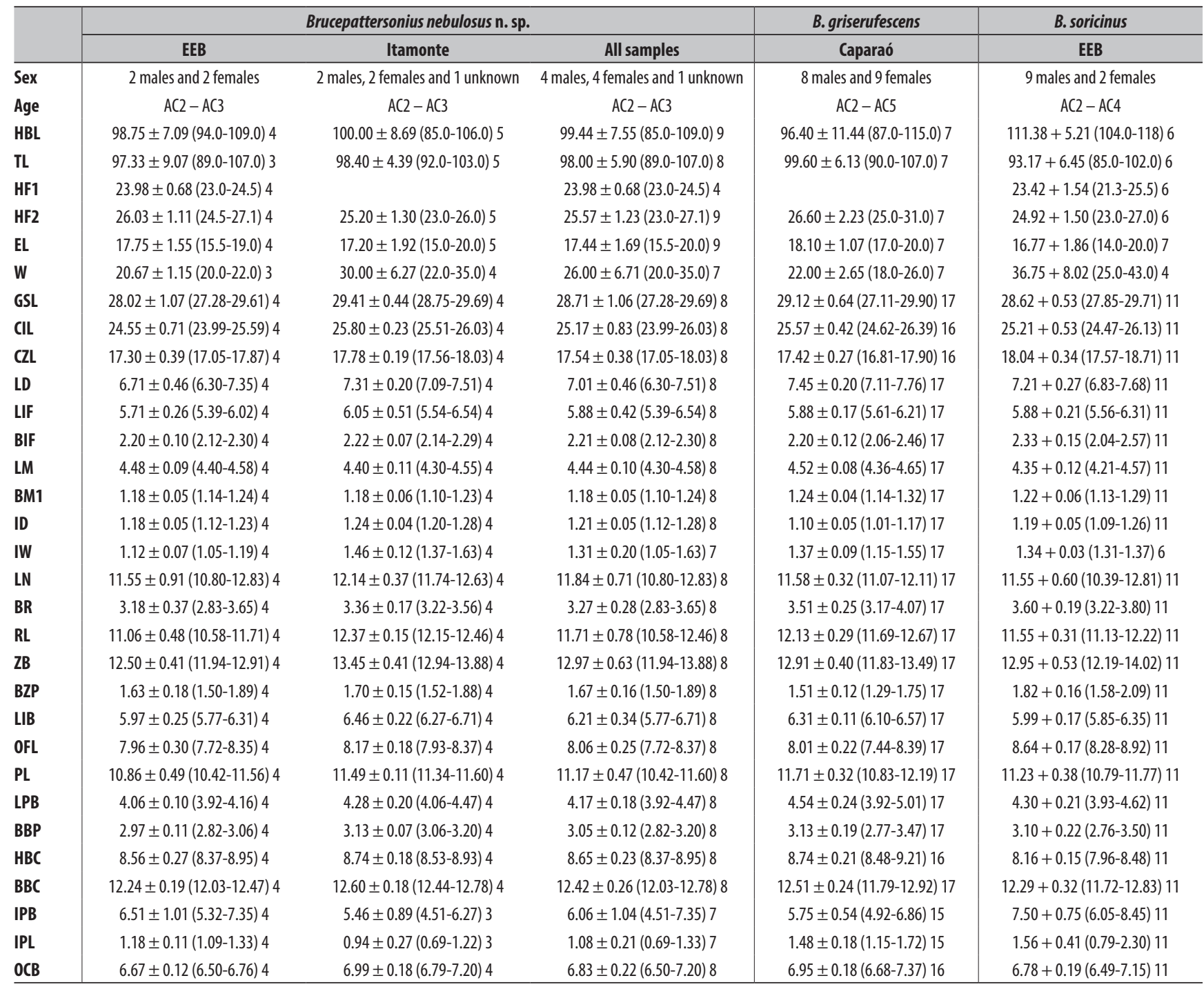

soft, dark brown; dorsal guard hairs long (length ranging from 9.5 to $11 \mathrm{~mm}$ ), with the basal portion ( $2 / 3$ ) narrower, grayish, and the apical third wider, brown in color; dorsal cover hairs medium (length ranging from 8 to $9 \mathrm{~mm}$ ), narrower basally and wider apically, grayish colored with light brown subterminal band and dark apical band; dorsal wool hairs slightly shorter (length about $8 \mathrm{~mm}$ ), thinner, grayish colored with a light brown subterminal band and a black terminal band. Lateral pelage grayish brown on the body and light brown on the head; limit between lateral and ventral color subtle, but evident. Ventral pelage color grayish-buff to slightly whitish; gular region grayish-buff tinged slightly with whitish; individual ventral hairs with basal portion (2/3) gray and apical portion ( $1 / 3)$ buff. Mystacial vibrissae short, hardly reaching the base of the pinnae when laid back. Pinnae small and rounded; covered internally and externally with numerous and short dark brown hairs. Dorsal surface of manus covered with brown to whitish hairs; digits with long claws (claw of dIII = $2.3 \mathrm{~mm} ; \mathrm{n}=3$ ). Pes moderately long and narrow, dorsally covered by grayish brown hairs; dIII longer than dII and similar to IV; digits with long claws (claw of dIII = $3 \mathrm{~mm} ; \mathrm{n}=3$ ); tufts of ungual hairs sparse and moderately long (few hairs extending beyond the claws), white colored. Tail similar or shorter than head and body length $(98 \% \pm 5.2$ of head and body length, ranging from 90.8 to $108.2 \%$; $n=8$ ); covered by short hairs, with length of about two scales at the basal portion of dorsal surface and length of about three scales at basal portion on ventral surface; scales arranged in 14 to 18 transverse rows per centimeter, at the base of the tail, between 15 to 17 rows per cm on the central portion of the tail, and between 20 and 22 rows on the apical centimeter of the tail $(n=2)$; dorsal tail color dark brown with dark brown hairs and ventral tail color dark brown slightly lighter at the base, with dark brown and few whitish hairs (specially at the base); tail predominantly unicolor.

Skull long and moderately broad, delicate (Fig. 26; Table 14). Rostrum very long and narrow, anterior portion of premaxillae partially apparent or not apparent in dorsal view. Nasals posteriorly long, extending beyond the maxillary-frontal-lacrimal suture, with posterior margin rounded or sharped. Premaxillae posteriorly long, extending beyond the nasals. Lacrimals small, usually with 


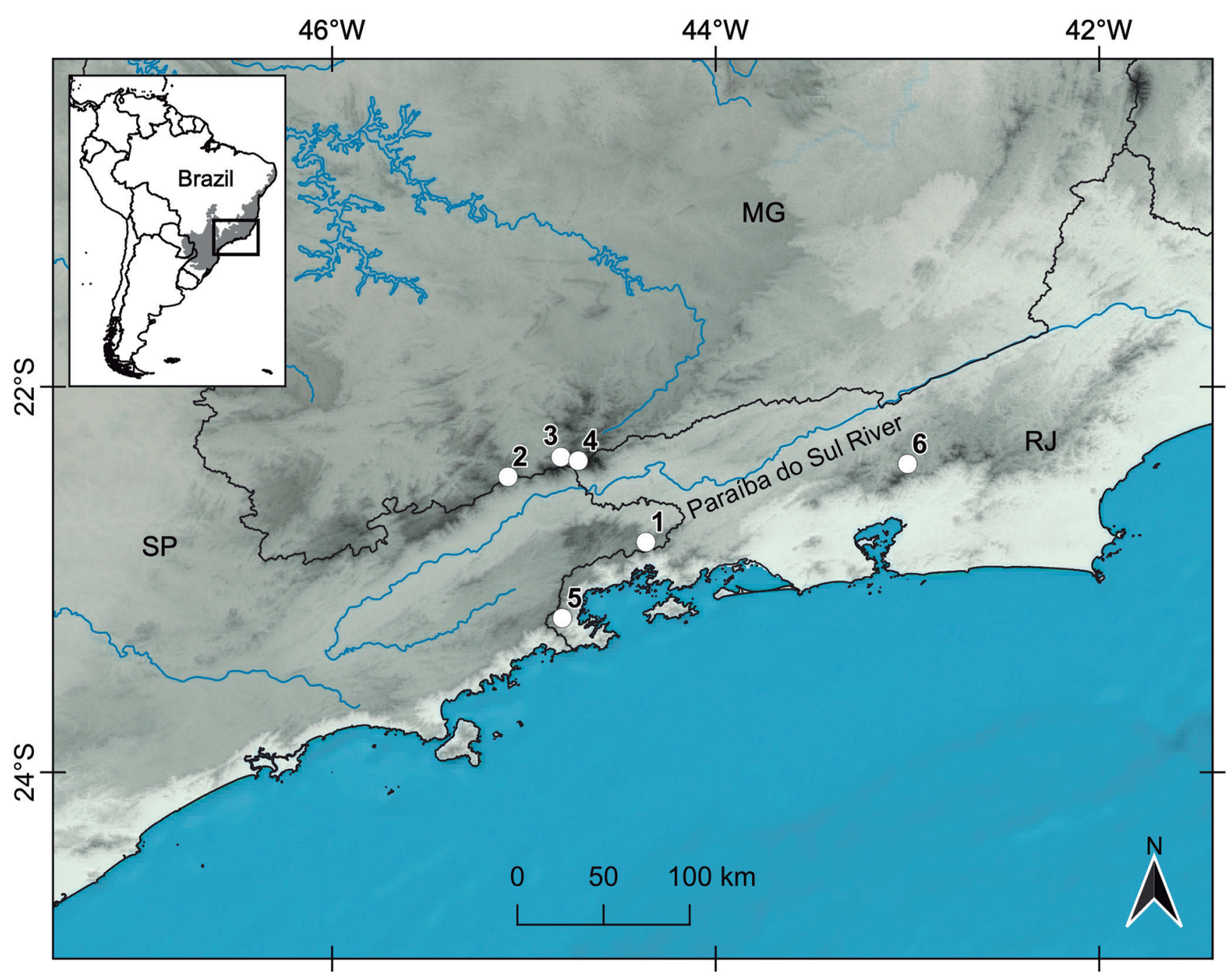

Figure 25. Geographic distributions of Brucepattersonius nebulosus n. sp. (1) Estação Ecológica de Bananal, Bananal, SP; (2) Fazenda do Itaguaré, 16 km SW, Passa Quatro, MG; (3) Casa Alpina, Itamonte, MG; (4) Brejo da Lapa, Itatiaia, RJ; (5) Parque Nacional da Serra da Bocaina, Paraty, RJ; (6) Parque Nacional da Serra dos Órgãos, Teresópolis, RJ. SP: São Paulo; MG: Minas Gerais; RJ: Rio de Janeiro.

equal contact with the maxillary and frontal or, in a few cases, in contact mainly with the maxillary. Zygomatic notch very shallow and narrow. Interorbital region broad, hourglass shaped; rounded and almost parallel supraorbital margins. Frontosquamosal suture not collinear or collinear with frontoparietal suture. Braincase moderately large and rounded, without temporal crests. Parietal partial or largely expanded to the lateral surface of the braincase. Nasals and premaxillae projected anteriorly beyond incisors, forming a distinct and long rostral tube. Gnathic process well developed. Zygomatic plate narrow and greatly sloped posteriorly with anterior margin rounded; free dorsal margin inconspicuous; dorsal root of the zygomatic process of the maxilla very narrow; posterior margin of zygomatic plate posterior to molar series. Zygomatic arch slender and delicate, nearly parallel; jugal present and large, maxillary and squamosal processes of zygomatic arch not overlapping. Anterior opening of alisphenoid canal present. Alisphenoid strut absent; buccinator-masticatory foramen and accessory oval foramen confluent. Squamosal-alisphenoid groove present on braincase lateral view, in the squamosal and alisphenoid bones; sphenofrontal foramen present; pos- terior opening of alisphenoid canal large; stapedial foramen present and large in the ectotympanic bullae, on the petrosal-ectotympanic suture (stapedial and carotid circulatory pattern 1; Voss, 1988). Subsquamosal fenestra present and wide. Postglenoid foramen large. Hamular process of squamosal narrow. Auditory bulla with large and rounded external auditory meatus; tegmen tympani greatly overlapped to posterior suspensory process of squamosal. Mastoid rounded and perforated by a medium size fenestra. Incisive foramina long, posterior margin surpassing the anterior face of $\mathrm{M} 1$ and reaching the protoflexus; anterior portion of incisive foramina very narrow, central region broad and posterior region narrow, but not as narrow as anterior region. Palate long and wide, with shallow lateral excavations and slightly deep in the central region, mainly on the anterior portion; posterolateral palatal pits usually present as one or two small perforations on each side. Mesopterygoid fossa wide, reaching the posterior face of $M 3$; sphenopalatine vacuities variable: present as large openings along the presphenoid reaching basisphenoid, or vacuities present but reduced as narrow openings, anterior to basisphenoid-presphenoid suture, or vacuities absent, 


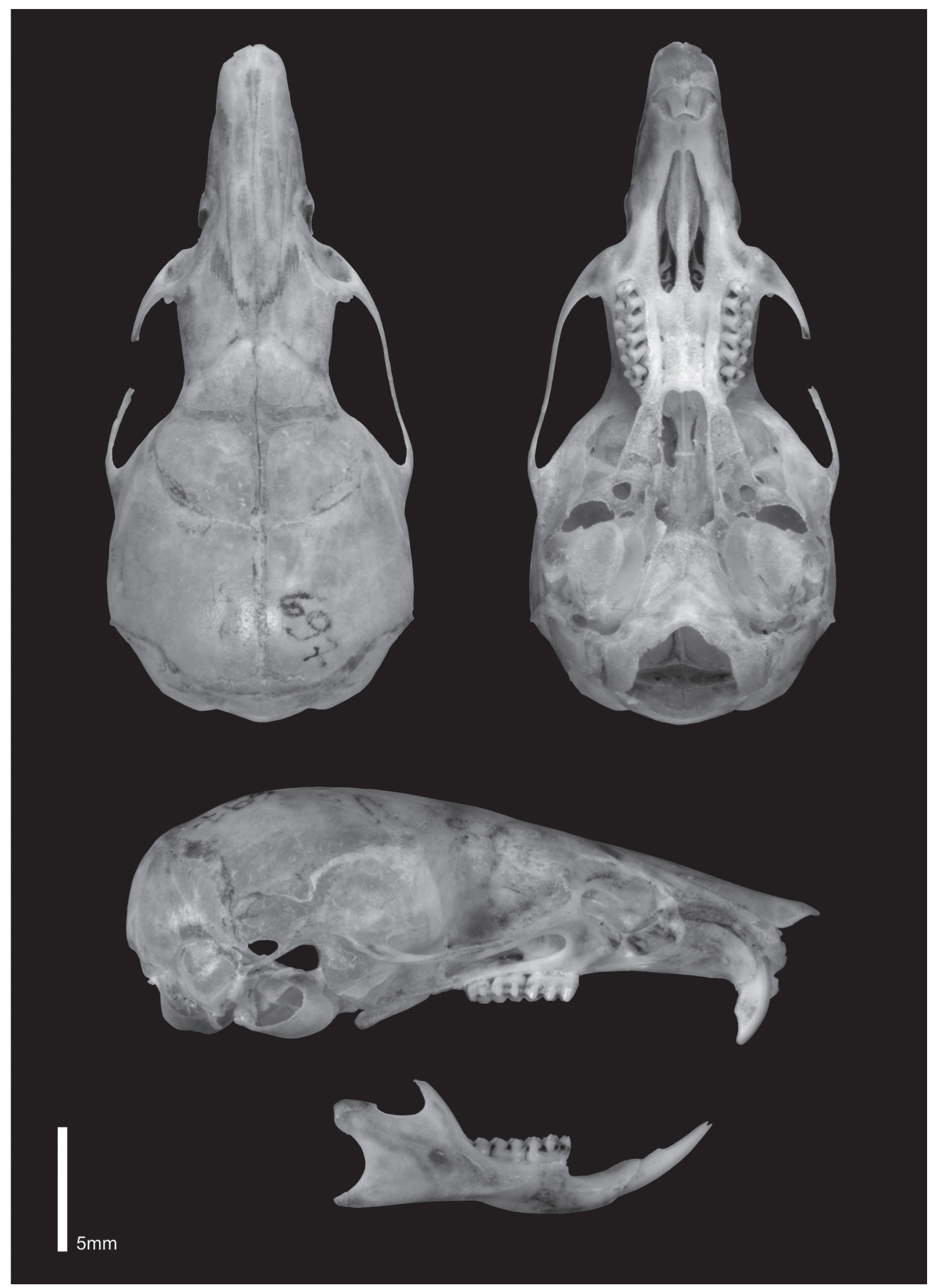

Figure 26. Skull and mandible of the holotype of Brucepattersonius nebulosus n. sp. (MZUSP 35847; male; GSL = $27.53 \mathrm{~mm}$ ). Skull on dorsal, ventral, and lateral view, and mandible on lateral view. 
mesopterygoid roof totally ossified. Parapterygoid plate narrower than the middle portion of mesopterygoid fossae. Stapedial process absent or very short. Auditory bullae small; exposed flange of periotic extends to internal carotid canal. Mandible elongated, with ramus very shallow (Fig. 26). Coronoid process usually sharp and slightly curved, higher than condyloid process. Angular process not surpassing the condyloid process. Superior and posterior notches deep. Capsular process of lower incisor alveolus absent. Superior and inferior masseteric ridges weak, especially the superior; anterior portion of masseteric ridges conjoined as single crest. Upper incisors opisthodont. Maxillary toothrows weakly divergent anteriorly. M1 with anterocone divided into labial and lingual conules by the anteromedian flexus; anteroloph very reduced as a projection of anterolabial conule reaching the paracone; protostyle absent; mesoloph reduced as a projection of paracone reaching the metacone; enterostyle absent or present. $\mathrm{m} 1$ with anteroconid without division, anteromedian flexid absent; anterolophid absent; mesolophid present, but reduced; protolophid and protostylid present; ectolophid and ectostylid absent or present.

Cytogenetics: Karyological preparations were not carried on EEB specimens, but based on Bonvicino et al. (1998) analyses from specimens sampled in Itamonte, Minas Gerais State, Brucepattersonius nebulosus shows the only exception from the stable $2 \mathrm{n}=52, \mathrm{NFa}=52$ found in the genus (Svartman \& Cardoso de Almeida, 1993; Mares \& Braun, 2000). This exception is based on a specimen that presented a NFa $=53$, due to a pair of small metacentric chromosomes that showed an inversion polymorphism. Besides this oddity, Brucepattersonius nebulosus presents the regular 24 pairs of acrocentric chromosomes that varies from large to small, and a small pair of metacentric chromosomes in its autosomal complement. The $\mathrm{X}$ chromosome is a medium acrocentric as $Y$ chromosome is a small acrocentric.

Remarks: Vilela (2005) analyzed the molecular (CYTB gene) and morphometric (cranio-dental) variation across the genus Brucepattersonius. He recognized five distinct taxonomic unities within the genus, among them, one unity including two specimens analyzed here: UFMG 1891 (from Passa Quatro, MG) and LG 108 (from Itatiaia, RJ). Vilela (2005) was the first to hypothesized that this lineage represented an undescribed species, which he called Brucepattersonius sp. "B". Later, in a more conservative posture, Vilela et al. (2015) allocated these very specimens under $B$. griserufescens, although still acknowledging them as a putative new species. Recently, Dias (2016), studding the molecular systematics, biogeography and diversification of Brucepattersonius, also analyzed the aforementioned specimens and found them consistently composing a distinct lineage within the genus (named Brucepattersonius sp.). Based on a robust molecular dataset, including five mitochondrial and nuclear markers (e.g., CYTB, BFIB, INS, ADH E IRBP), Dias (2016), suggested that this lineage represented an unnamed species, which deserved a formal description.
Additional specimens examined: Brucepattersonius griserufescens: $\mathrm{n}=18$; Brazil: Espírito Santo: Parque Nacional do Caparaó, Dores do Rio Preto $\left(20^{\circ} 28^{\prime} 49^{\prime \prime} S\right.$, 4149'46"W): female: UFES 1134, 1135, 1137, 1140, 1161, 1162, 1164; male: UFES 1131, 1132, 1133, 1136, 1138, 1139, 1163, 1165; sex unknown: UFES 1128; Minas Gerais: Parque Nacional do Caparaó, Pico da Bandeira (20²5'S, 41ํㄱ'W): female: MN 32016, 32017.

Field observations: The individuals of Brucepattersonius nebulosus collected at EEB were captured with pitfall traps in December, 2003 (three specimens) and in April, 2004 (one specimen). Specimens captured in December, 2003, two females and one male, did not show signs of reproductive activity: the male had abdominal testes and the females had unperforated vaginas.

\section{Brucepattersonius soricinus Hershkovitz 1998}

Voucher material: $\mathrm{n}=11$; female: uncatalogued: EBM 078; EEB 752; male: MZUSP 33713, 33758; uncatalogued: EBM 089, 122, 219, 307; EEB 764, 893, 902.

Specimens identification: Medium size (see body measurements on Table 14). Dorsal pelage dense and soft, brownish or copperish-brown in some adult specimens; dorsal guard hairs long (length about $11 \mathrm{~mm}$ ), with the basal portion $(2 / 3)$ narrower, gray in color, and the apical third wider, brownish; dorsal cover hairs medium (length about $9 \mathrm{~mm}$ ), narrower basally and wider apically (half and half), grayish colored with light brown subterminal band and black apical band; dorsal wool hairs slightly shorter (length about $8 \mathrm{~mm}$ ), thinner, grayish colored with a light brown subterminal band and a black terminal band. Lateral pelage predominantly brown colored in the body, grayish in some young specimens and copperish in some adult specimens, and lighter on the head; limit between lateral and ventral color indistinct. Ventral pelage color usually grizzled orange/copperish; gular region orange colored to copperish in some older specimens; individual ventral hairs with basal portion ( $2 / 3$ ) light gray colored and apical portion ( $1 / 3$ ) orange/copperish. Mystacial vibrissae short, not reaching the base of the pinnae when laid back. Pinnae small and rounded; covered internally and externally with numerous and short brownish hairs. Dorsal surface of manus covered mainly by whitish hairs, some of those with gray basal portion; digits with long claws (claw of dIII $=2.5 \mathrm{~mm} ; \mathrm{n}=1$ ). Pes moderately long and narrow, dorsally covered by numerous whitish hairs; dIII longer than others; digits with long claws (claw of dIII = $3.0 \mathrm{~mm} ; \mathrm{n}=1$ ); tufts of ungual hairs sparse and short, usually not extending beyond the claws, white colored. Tail shorter than head and body length $(82.5 \% \pm 5.4$ of head and body length, ranging from 75.9 to $92.7 \% ; n=8$ ); covered by short hairs, with length of one to two scales, at the basal portion of tail on both surfaces; scales arranged in 15 transverse rows per centimeter, at the base of the tail, 14 rows per $\mathrm{cm}$ on the central portion of the tail, and 18 rows on the apical 
centimeter of the tail $(n=1)$; dorsal tail color brown with brown hairs and ventral tail color light brown with hairs brown colored on the base and whitish on the apical portion; tail predominantly bicolor.

Skull long and moderately broad (Fig. 27; Table 14). Rostrum very long and broad, anterior portion of premaxillae usually apparent in dorsal view. Nasals posteriorly short, not extending beyond the maxillary-frontal-lacrimal suture (54.5\%) or long, surpassing the maxillary-frontal-lacrimal suture $(45.5 \%)(n=11)$; posterior margin of nasals sharp $(54.5 \%)$ or rounded $(45.5 \%)$ $(n=11)$. Premaxillae in general posteriorly short, ending before nasals $(72.7 \%)$, or ending collinearly with nasals (27.3\%) ( $n=11)$. Lacrimals usually well developed, with equal contact with the maxillary and frontal (63.6\%) or in contact mainly with the maxillary $(36.4 \%)(n=11)$. Zygomatic notch deep and broad. Interorbital region broad, hourglass shaped; rounded supraorbital margins. Frontosquamosal suture not collinear $(90.9 \%)$ or collinear $(9.1 \%)$ with frontoparietal suture $(n=11)$. Braincase moderately large and rounded, without temporal crests. Parietal partial expanded to the lateral surface of braincase $(63.6 \%)$, largely expanded $(18.2 \%)$ or not expanded to the lateral surface of braincase $(18.2 \%)(n=11)$. Nasals and premaxillae projected anteriorly beyond incisors, forming a distinct and long rostral tube. Gnathic process well developed. Zygomatic plate narrow and greatly sloped posteriorly with anterior margin rounded; free dorsal margin inconspicuous; dorsal root of the zygomatic process of the maxilla broad; posterior margin of zygomatic plate collinear to molar series. Zygomatic arch slender and delicate, nearly parallel; jugal present and large, maxillary and squamosal processes of zygomatic arch not overlapping. Anterior opening of alisphenoid canal present. Alisphenoid strut absent, buccinator-masticatory foramen and accessory oval foramen confluent (90.9\%), or present, buccinator-masticatory foramen and accessory oval foramen not confluent $(9.1 \%)(n=11)$. Squamosal-alisphenoid groove present on braincase lateral view, in the squamosal and alisphenoid bones; sphenofrontal foramen present; posterior opening of alisphenoid canal large; stapedial foramen present and large in the ectotympanic bullae, on the petrosal-ectotympanic suture (stapedial and carotid circulatory pattern 1; Voss, 1988). Subsquamosal fenestra present and usually narrow. Postglenoid foramen usually small. Hamular process of squamosal broad. Auditory bulla with large and rounded external auditory meatus; tegmen tympani greatly overlapped to posterior suspensory process of squamosal. Mastoid rounded and perforated by a medium size fenestra. Incisive foramina long, posterior margin surpassing the anterior face of M1 (54.5\%) or only reaching the anterior face of $M 1(45.5 \%)(n=11)$; anterior portion of incisive foramina usually very narrow and sharp, central region broader and posterior region subtly narrower or equal to central portion. Palate long and wide, with shallow lateral excavations and slightly deep in the central region, mainly on the anterior portion; posterolateral palatal pits present as one small perforation on each side (63.6\%), present as more than one pit on at least one side (27.3\%) or absent $(9.1 \%)(n=11)$. Mesopterygoid fossa wide; reaching the posterior face of M3 (63.6\%), surpassing the posterior face of M3 (18.2\%) or not reaching the posterior face of M3 (18.2\%) $(n=11)$; sphenopalatine vacuities present as large openings along the presphenoid reaching basisphenoid (81.8\%), or vacuities present but reduced as narrow openings, anterior to basisphenoid-presphenoid suture (9.1\%), or vacuities absent, mesopterygoid roof totally ossified (9.1\%) $(n=11)$. Parapterygoid plate with breadth similar to the middle portion of mesopterygoid fossae $(72.7 \%)$ or narrower than the middle portion of mesopterygoid fossae $(27.3 \%)(n=11)$. Stapedial process long and thin. Auditory bullae small; exposed flange of periotic extends to internal carotid canal. Mandible elongated, with ramus very shallow (Fig. 27). Coronoid process usually rounded and slightly curved, higher than condyloid process. Angular process not surpassing the condyloid process. Superior and posterior notches slightly deep. Capsular process of lower incisor alveolus absent. Superior and inferior masseteric ridges with the anterior portion conjoined as single crest. Upper incisors opisthodont. Maxillary toothrows weakly divergent anteriorly. M1 with anterocone undivided, anteromedian flexus absent (54.5\%), or partially divided by the internal fold of procingulum (36.4\%), or divided into labial and lingual conules by the anteromedian flexus $(9.1 \%)(n=11)$; anteroloph very reduced as a projection of anterolabial conule reaching the paracone $(87.5 \%)$ or absent $(12.5 \%)(n=8)$; protostyle absent $(88.9 \%)$ or present $(11.1 \%)(n=9)$; mesoloph present and very reduced as a projection of paracone reaching the metacone $(88.9 \%)$ or absent $(11.1 \%)(n=9)$; enterostyle absent. $\mathrm{m} 1$ with anteroconid without division, anteromedian flexid absent (66.7\%), or anteroconid divided into labial and lingual conulids by the anteromedian flexid (33.3\%) ( $n=9)$; anterolophid absent; mesolophid present but reduced $(88.9 \%)$ or absent $(11.1 \%)(n=9)$; protolophid and protostylid present $(77.8 \%)$ or absent (22.2\%) ( $n=9)$; ectolophid and ectostylid absent $(66.7 \%)$ or present $(33.3 \%)(n=9)$.

Remarks: In the original description of $B$. soricinus, the morphological description is very brief, especially regarding the ontogenetic variation (Hershkovitz, 1998). In the EEB specimens, we notice strong age-related variation, particularly in the pelage coloration. Young specimens show a general pattern of grayish color and old specimens present a reddish copper pattern. This observation is particularly relevant because the pelage color was proposed by Hershkovitz (1998) as one of the key characteristics to distinguish $B$. soricinus from $B$. igniventris. The pelage pattern described here for young specimens of $B$. soricinus corresponds with the original description of the species, while the pelage color of adults matches the diagnose provided by Hershkovitz (1998) for B. igniventris.

Field observations: The 11 specimens of $B$. soricinus collected at EEB were captured in December, 2003 (four individuals), and April (five individuals) and December 


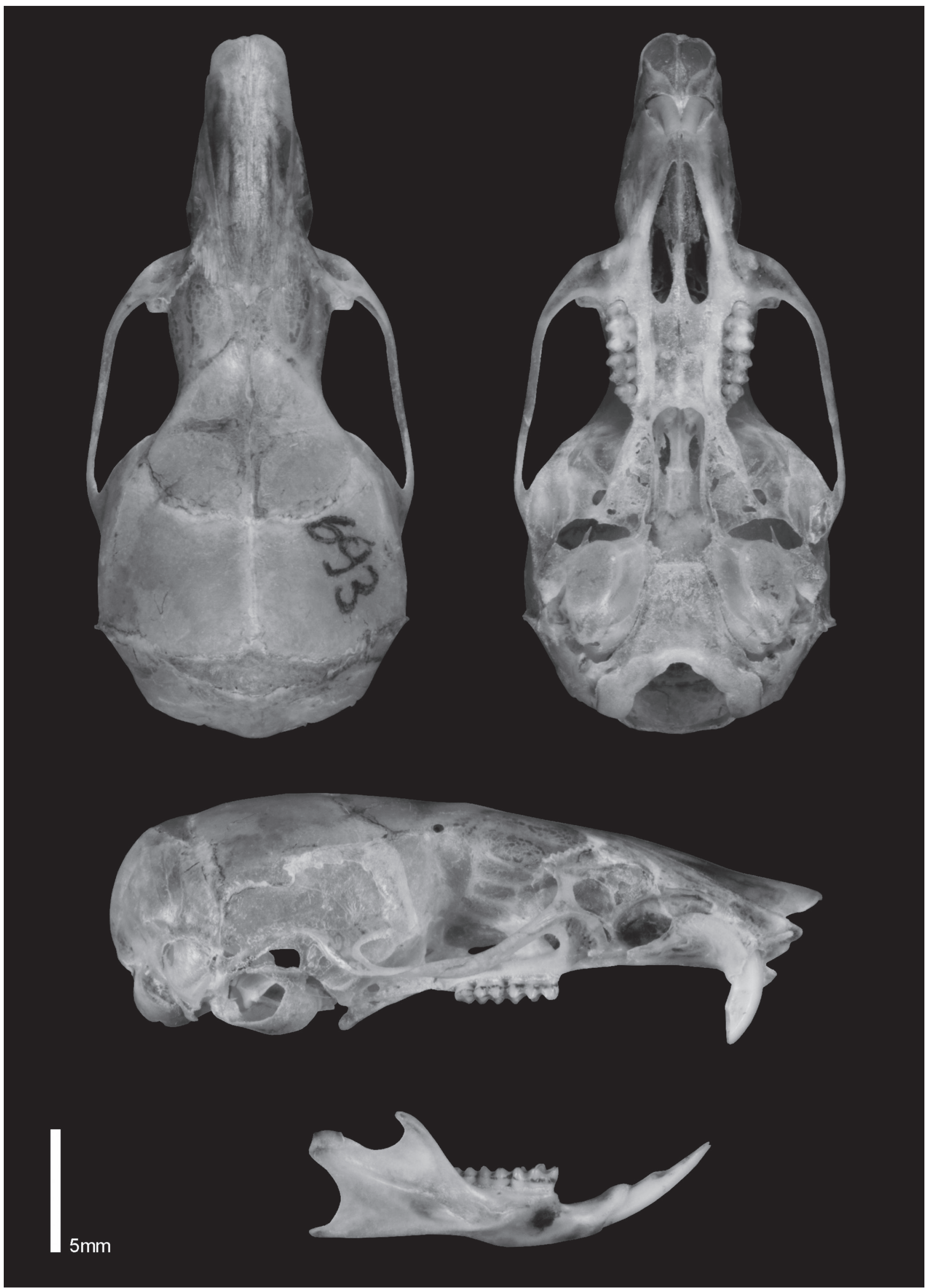

Figure 27. Skull and mandible of Brucepattersonius soricinus (MZUSP 33758; male; GSL = $28.95 \mathrm{~mm}$ ). Skull on dorsal, ventral, and lateral view, and mandible on lateral view. 
(two individuals), 2004, all in pitfall traps. The four individuals captured in December, 2003 showed signs of reproductive activity: three males with scrotal testes and one female with mammal glands visible and perforated vagina. In April 2004, three individuals showed signs of reproductive activity: two males with scrotal testes and one female with perforated vagina. In December 2004, information about the reproductive state of specimens was not taken.

\section{Castoria angustidens (Winge, 1887)}

Voucher material: $n=6$; female: MZUSP 33694, 33705; male: uncatalogued: EBM 032, 180, 336; EEB 825.

Specimens identification: Small size (see body measurements on Table 15). Dorsal pelage dense and soft, dark brown speckled with ferruginous yellow hairs; dorsal guard hairs long (length about $11 \mathrm{~mm}$ ), with the basal portion (3/4) narrower, gray in color, and the apical portion ( $1 / 4)$ wider, black in color; dorsal cover hairs medium (length about $9 \mathrm{~mm}$ ), dark gray colored with the apical portion (about $1 / 6$ ) composed by three bands of color, a basal brown band, a subterminal ferruginous yellow band and a apical brown band; dorsal wool hairs short (length about $6 \mathrm{~mm}$ ), thinner in the basal portion and slightly wider in the apex, dark gray colored. Lateral pelage mainly brownish colored speckled with ferruginous yellow hairs, more intense near the head; limit between lateral and ventral color very subtle, almost imperceptible. Ventral pelage color ferruginous slightly grayish; individual ventral hairs with basal portion (two thirds) gray and apical portion (one third) ferruginous. Mystacial vibrissae short, a few hairs reaching the base of the pinnae when laid back. Pinnae small and rounded; covered externally by numerous hairs, grayish colored, and internally by less numerous hairs, dark brown colored on the basal portion and ferruginous on the apical portion. Dorsal surface of manus covered by dark brown hairs; digits with medium claws (claw of dIII about $2 \mathrm{~mm}$ longer; $n=1$ ). Pes short and narrow, dorsally covered by hairs brown colored on the base and lighter brown on top; dIII similar in length to dII and dIV; digits with medium claws (claw of dIII about $2.85 \mathrm{~mm}$ longer; $\mathrm{n}=1$ ); tufts of ungual hairs scarce and short, few hairs extending beyond the claws, brownish colored. Tail slightly shorter than head and body $(92.7 \% \pm 6.2$ of head and body length, ranging from 90.8 to $99.6 \% ; n=3$ ); covered by short hairs, with length of one to two scales at the basal portion on both surfaces; scales arranged in 20 transverse rows per centimeter at the basal and central portion of the tail and in 23 rows on the apical centimeter of the tail $(n=1)$; dorsal tail color dark brown, with dark brown hairs, and ventral tail color lighter brown, with hairs brown colored on the basal portion and whitish on the apical portion; tail generally unicolor.

Skull elongated and slightly wide (Fig. 28; Table 15). Rostrum moderately long. Nasals anteriorly long, extending beyond the incisors, and posteriorly short, not
Table 15. Descriptive statistics (mean \pm standard deviation, range, and sample size) of body and cranio-dental measurements $(\mathrm{mm})$, and weight $(\mathrm{g})$, of adult specimens of Castoria angustidens from EEB, and descriptive statistics for the species obtained in Christoff et al. (2000).

\begin{tabular}{|c|c|c|}
\hline & $\begin{array}{c}\text { Species values } \\
\text { (13 males and } 2 \text { females) }\end{array}$ & $\begin{array}{c}\text { EEB specimens } \\
\text { (4 males and } 2 \text { females) }\end{array}$ \\
\hline HBL & $97.6(90.5-110.0) 9$ & $89.62 \pm 5.95(85.0-100.9) 5$ \\
\hline TL & $84.8(76.0-98.5) 9$ & $80.93 \pm 2.66(79.0-84.7) 3$ \\
\hline HF2 & - & $25.68 \pm 1.29(23.8-27.0) 5$ \\
\hline HF1 & $23.3(22.0-25.0) 9$ & $24.18 \pm 1.24(23.0-25.5) 5$ \\
\hline EL & $15.2(12.5-17.0) 9$ & $16.70 \pm 1.96(13.5-19.0) 5$ \\
\hline w & - & $22.90 \pm 4.93(19.0-32.5) 5$ \\
\hline GSL & - & $26.83 \pm 0.41(26.38-27.30) 4$ \\
\hline CIL & $25.19(23.38-26.67) 16$ & $23.70 \pm 0.36(23.17-23.95) 4$ \\
\hline CZL & $18.30(17.06-19.16) 16$ & $17.19 \pm 0.28(16.91-17.57) 4$ \\
\hline LD & $7.29(6.51-7.91) 16$ & $6.70 \pm 0.31(6.27-6.93) 4$ \\
\hline LIF & $6.11(5.53-6.95) 16$ & $5.72 \pm 0.23(5.51-6.05) 4$ \\
\hline BIF & - & $2.17 \pm 0.07(2.09-2.24) 4$ \\
\hline LM & $4.96(4.65-5.31) 16$ & $4.97 \pm 0.18(4.86-5.18) 3$ \\
\hline BM1 & $1.22(1.10-1.35) 15$ & $1.14 \pm 0.09(1.02-1.23) 4$ \\
\hline ID & - & $1.15 \pm 0.10(1.07-1.30) 4$ \\
\hline IW & - & $1.33 \pm 0.05(1.27-1.40) 4$ \\
\hline LN & - & $10.56 \pm 0.40(10.12-11.09) 4$ \\
\hline BR & - & $3.62 \pm 0.13(3.45-3.74) 4$ \\
\hline RL & $10.79(9.78-11.57) 15$ & $10.39 \pm 0.27(10.22-10.79) 4$ \\
\hline ZB & $14.50(13.53-15.03) 14$ & $13.55 \pm 0.21(13.31-13.80) 4$ \\
\hline BZP & $2.70(2.40-2.97) 16$ & $2.66 \pm 0.11(2.58-2.82) 4$ \\
\hline LIB & $5.38(5.14-5.70) 16$ & $5.47 \pm 0.08(5.41-5.59) 4$ \\
\hline OFL & $8.55(8.19-9.00) 16$ & $7.91 \pm 0.29(7.55-8.26)$ \\
\hline PL & - & $11.29 \pm 0.23(11.05-11.60) 4$ \\
\hline LPB & $4.12(3.81-4.41) 15$ & $4.25 \pm 0.19(4.12-4.53) 4$ \\
\hline BBP & $2.84(2.52-3.31) 16$ & $2.60 \pm 0.10(2.49-2.68) 3$ \\
\hline HBC & - & $8.61 \pm 0.29(8.25-8.87) 4$ \\
\hline BBC & - & $12.55 \pm 0.08(12.46-12.66) 4$ \\
\hline IPB & - & $7.77 \pm 0.60(6.91-8.28) 4$ \\
\hline IPL & - & $2.45 \pm 0.21(2.17-2.68) 4$ \\
\hline OCB & $6.42(6.04-6.83) 16$ & $6.45 \pm 0.26(6.08-6.67) 4$ \\
\hline
\end{tabular}

extending beyond the maxillary-frontal-lacrimal suture (three specimens) or long, extending beyond the maxillary-frontal-lacrimal suture (one specimen); with posterior margin rounded (three specimens) or sharp (one specimen); nasals narrow, premaxillae visible on dorsal view. Premaxillae short, ending anteriorly to nasals. Lacrimal with equal proportion of contact with maxillary and frontal (three specimens) or in contact mainly with maxillary (one specimen). Zygomatic notch from moderate to deep. Interorbital region hourglass in shape with rounded supraorbital margins (two specimens) or with supraorbital margins posteriorly squared (two specimens). Frontosquamosal suture not collinear with frontoparietal suture and situated anteriorly to this. Braincase large, wide and slightly elongated; temporal crests absent. Parietal partial expanded to the lateral surface of the braincase. Zygomatic plate short and broad, with anterodorsal margin produced as a sharp corner and conspicuously anterior to superior maxillary root of zygoma; posterior margin of zygomatic plate situated posteriorly to the alveolus of M1. Jugal present and large, maxillary and squamosal 


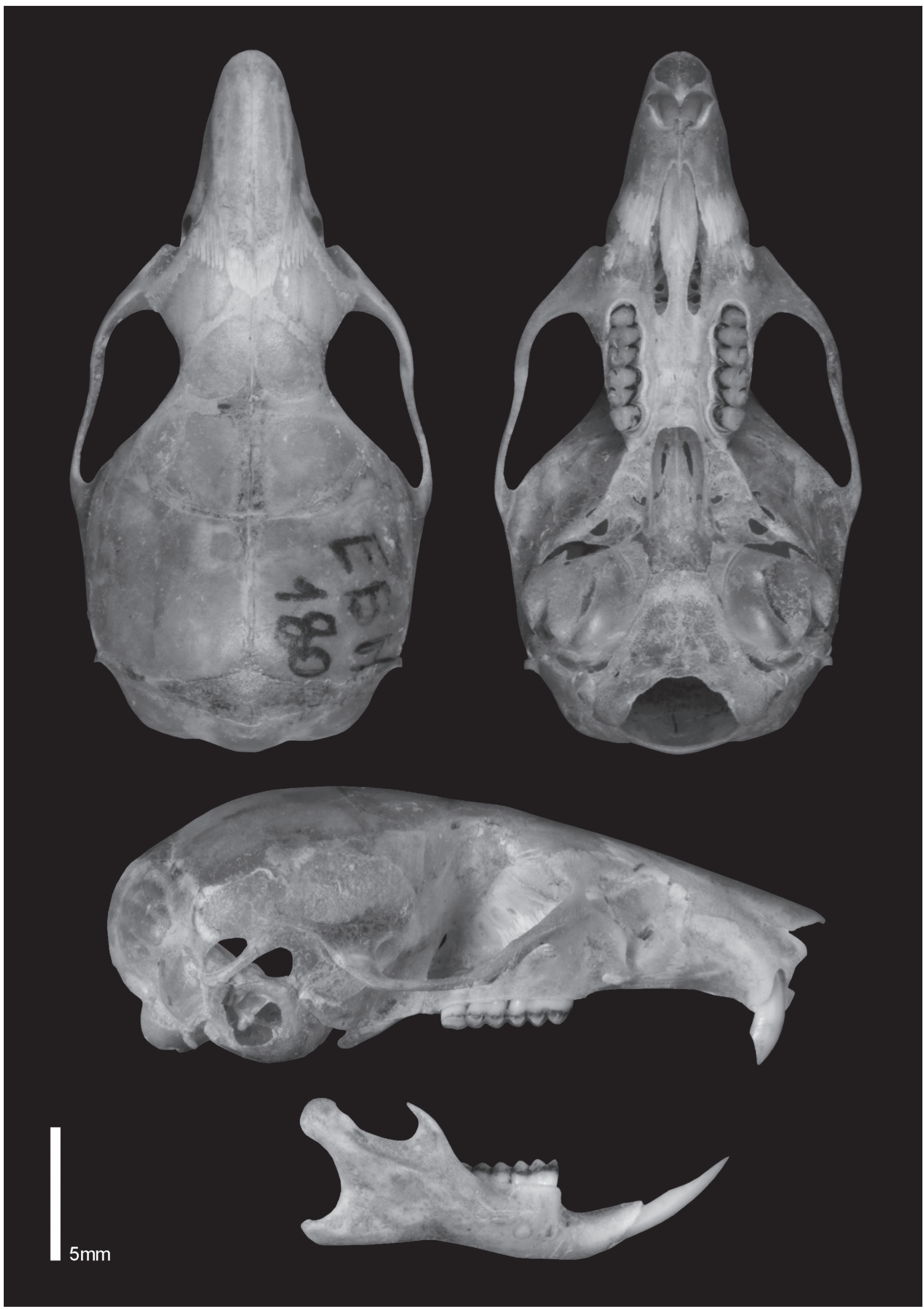

Figure 28. Skull and mandible of Castoria angustidens (EBM 180; male; $G S L=26.61 \mathrm{~mm}$ ). Skull on dorsal, ventral, and lateral view, and mandible on lateral view. 
processes of zygomatic arch not overlapping. Anterior opening of alisphenoid canal present. Alisphenoid strut present, buccinator-masticatory foramen and accessory oval foramen separate. Squamosal-alisphenoid groove present on braincase lateral view, in the squamosal and alisphenoid bones; sphenofrontal foramen present; posterior opening of alisphenoid canal large; stapedial foramen present and large in the ectotympanic bullae, on the petrosal-ectotympanic suture (stapedial and carotid circulatory pattern 1; Voss, 1988). Subsquamosal fenestra present. Mastoid with conspicuous fenestra. Incisive foramina teardrop on shape, with anterior portion narrow, central region broad and posterior portion slightly narrower than central region; posterior margin of incisive foramina surpassing the anterior face of M1 and reaching the protoflexus (three specimens) or just reaching the anterocone (one specimen). Palate short and wide, usually flat; lateral excavations shallow and one central foramen present on each side; posterolateral palatal pits present as one small opening on each side of posterior palate. Mesopterygoid fossa just reaching the posterior face of M3 (three specimens) or surpassing the posterior face of $\mathrm{M} 3$ (one specimen); sphenopalatine vacuities present as large openings along the presphenoid reaching basisphenoid. Parapterygoid plate with similar width to the middle portion of mesopterygoid fossae (two specimens), narrower than the middle portion of mesopterygoid fossae (one specimen), or wider than the middle portion of mesopterygoid fossae (one specimen). Auditory bullae intermediate, exposed wedge of periotic small and not contributing to wall of carotid canal. Mandible elongated, with ramus moderately deep (Fig. 28). Superior notch deep and posterior notch moderately shallow. Capsular process of lower incisor alveolus absent. Superior and inferior masseteric ridges with anterior portion conjoined as single crest. Upper incisors opisthodont. Maxillary toothrows slightly anteriorly divergent. $\mathrm{M} 1$ with anterocone not divided into labial and lingual conules, anteromedian flexus absent; anteroloph present but reduced as a projection of anterolabial conule; mesoloph present but reduced as a projection of paracone. $\mathrm{m} 1$ with anteroconid not divided into labial and lingual conulids, anteromedian flexid absent; anterolophid absent; protostylid present and well developed; mesolophid absent; entoconid with entolophulid; ectolophid and ectostylid absent.

Field observations: Six C. angustidens were collected at EEB, two females and four males. The two females had unperforated vaginas when they were captured, in December, 2003. The four males were captured in April and May, 2004, and there is no information about their reproductive status. Five specimens were captured by pitfall traps and one with Sherman trap.

\section{Oxymycterus dasytrichus (Schinz 1821)}

Voucher material: $\mathrm{n}=2$; male: uncatalogued: EEB 806, 850.
Specimens identification: Large size. Dorsal pelage dense and moderately rough, reddish dark brown, slightly darker on mid-dorsal region; dorsal guard hairs long (length ranging from 13 to $14.5 \mathrm{~mm}$ ), with the basal half narrower, dark brown, and the apical half wider, brown in color; dorsal cover hairs medium (length ranging from 10.5 to $12 \mathrm{~mm}$ ), darkish colored with a copperish orange subterminal band and a darkish apical band; dorsal wool hairs short (length ranging from 7.5 to $9 \mathrm{~mm}$ ), entirely gray colored. Lateral pelage cooperish brown colored, lighter than dorsum; limit between lateral and ventral color delimited. Ventral pelage ranging from ocher gray to orange ocher; individual ventral hairs with basal portion (from $1 / 2$ to $1 / 3$ ) gray colored and apical portion (from $1 / 2$ to $2 / 3$ ) ocher or orange ocher colored. Mystacial vibrissae short, a few hairs reaching the base of the pinnae when laid back. Pinnae large and notably rounded; covered internally with numerous hairs principally on basal region and externally with numerous hairs mostly on apical region, dark brown colored on both surfaces. Dorsal surface of manus covered by hairs with brownish base and whitish top; digits with very long claws (claw of dIII with length ranging from 4.8 to $5.3 \mathrm{~mm} ; \mathrm{n}=2$ ). Pes moderately long and wide, dorsally covered by numerous hairs, dark brown colored; dIII slightly longer than dII and dIV; digits with very long claws (claw of dIII ranging from 4.6 to $4.9 \mathrm{~mm}$; $\mathrm{n}=2$ ); tufts of ungual hairs short, about half of claw's length, brown colored. Tail shorter than head and body; covered by short hairs, with length of one to two scales at the basal portion on both surfaces; scales arranged in 16 transverse rows per centimeter at the base of the tail, between 12 to 14 rows per $\mathrm{cm}$ on the central portion of the tail, and in 13 rows on the apical centimeter of the tail $(n=2)$; dorsal tail color dark brown, with dark brown hairs, and ventral tail color dark brown slightly lighter, with hair basally brown and apically whitish; tail generally unicolor.

Skull long and narrow (Fig. 29; Table 16). Rostrum very long. Nasals posteriorly short, not extending beyond the maxillary-frontal-lacrimal suture; posterior margin of nasals rounded. Premaxillae posteriorly short, ending before nasals. Lacrimals with equal contact with the maxillary and frontal. Zygomatic notch deep. Interorbital region broad, hourglass shaped; supraorbital margins rounded anteriorly and squared posteriorly. Frontosquamosal suture collinear (one specimen) or not collinear (one specimen) with frontoparietal suture. Braincase narrow and elongated, without temporal crests. Parietal partial expanded to the lateral surface of braincase. Nasals and premaxillae projected anteriorly beyond incisors, forming a distinct and long rostral tube. Gnathic process very reduced. Zygomatic plate narrow and sloped posteriorly with anterodorsal margin rounded and conspicuously anterior to superior maxillary root of zygoma; posterior margin of zygomatic plate situated posteriorly to the alveolus of M1. Jugal present and large, maxillary and squamosal processes of zygomatic arch not overlapping. Anterior opening of alisphenoid canal present. Alisphenoid strut present, buccinator-masticatory foramen and accessory oval foramen not confluent. 
Table 16. Cranio-dental morphometric values ( $\mathrm{mm}$ ) of Oxymycterus dasytrichus from EEB.

\begin{tabular}{|c|c|c|}
\hline & EEB 850 (subadult, male) & EEB 806 (adult, male) \\
\hline GSL & 36.17 & 37.92 \\
\hline CIL & 32.02 & 33.75 \\
\hline CZL & 22.37 & 23.22 \\
\hline LD & 8.24 & 9.20 \\
\hline LIF & 7.79 & 7.83 \\
\hline BIF & 2.71 & 3.55 \\
\hline LM & 5.62 & 5.61 \\
\hline BM1 & 1.43 & 1.54 \\
\hline ID & 1.47 & 1.76 \\
\hline IW & - & 1.74 \\
\hline LN & 14.26 & 14.91 \\
\hline BR & 4.39 & 4.66 \\
\hline RL & 14.84 & 15.50 \\
\hline ZB & 15.45 & 16.85 \\
\hline BZP & 2.37 & 2.94 \\
\hline LIB & 6.78 & 7.08 \\
\hline OFL & 10.31 & 10.92 \\
\hline PL & 13.33 & 14.13 \\
\hline LPB & 4.30 & 4.94 \\
\hline BBP & 3.36 & 3.99 \\
\hline HBC & 10.26 & 10.34 \\
\hline BBC & 14.64 & 15.30 \\
\hline IPB & 9.09 & 9.31 \\
\hline IPL & 2.95 & 3.55 \\
\hline$O C B$ & 8.40 & 8.26 \\
\hline
\end{tabular}

Squamosal-alisphenoid groove present on braincase lateral view, in the squamosal and alisphenoid bones; sphenofrontal foramen present; posterior opening of alisphenoid canal large; stapedial foramen present and large in the ectotympanic bullae, on the petrosal-ectotympanic suture (stapedial and carotid circulatory pattern 1; Voss, 1988). Subsquamosal fenestra present but vestigial. Mastoid completely ossified or with a diminutive pit in the dorsal contact with the exoccipital border. Incisive foramina long, posterior margin surpassing the anterior face of $\mathrm{M} 1$ and reaching the anterocone (one specimen) or reaching the protoflexus (one specimen); anterior portion of incisive foramina narrow, central and posterior regions broader and similar in width. Palate short and wide, with lateral excavations shallow anteriorly and deeper posteriorly; posterolateral palatal pits present as one or two openings on each side. Mesopterygoid fossa surpassing the posterior face of M3; sphenopalatine vacuities absent, mesopterygoid roof totally ossified. Parapterygoid plate narrower than the middle portion of mesopterygoid fossae. Auditory bullae intermediate, exposed wedge of periotic small and not contributing to wall of carotid canal. Mandible elongated, with ramus very low (Fig. 29). Superior notch shallow and posterior deep. Capsular process of lower incisor alveolus absent. Superior and inferior masseteric ridges with the anterior portion conjoined as single crest. Upper incisors opisthodont. Maxillary toothrows parallels. M1 with anterocone divided into labial and lingual conules by the anteromedian flexus; anteroloph reduced as a projection of anterolabial conule reaching the paracone; protostyle present; mesoloph present and reduced as a projection of paracone toward to the metacone; enterostyle present. $\mathrm{m} 1$ with anteroconid without division, anteromedian flexid absent; anterolophid and anteroflexid absent; protostylid present (one specimen) or absent (one specimen); mesolophid absent; ectostylid present (one specimen) or absent (one specimen).

Remarks: In the most recent taxonomic arrangement proposed for Oxymycterus, Oliveira \& Gonçalves (2015) recognized 15 species, five of which occur in the eastern Brazil, with three potentially occuring at EEB, i.e., $O$. dasytrichus, O. quaestor (including O. missionalis and $O$. judex as junior synonyms), and O. rufus. According to Gonçalves \& Oliveira (2004), O. dasytrichus is characterized by the dark dorsal coloration, interparietal large and expanded laterally, and lacrimal pronounced laterally characteristics observed in the EEB specimens. Therefore, it differs from $O$. rufus, which has predominantly reddish dorsal coloration, interparietal small and triangular and lacrimal reduced; and from 0 . quaestor, with has dorsal coloration dark yellow gray or dark orange. Furthermore, $O$. dasytrichus differs from both $O$. rufus and $O$. quaestor in the high frequency of presence of the alisphenoid strut, which is present on both sides in $90 \%$ of the specimens examined by Gonçalves \& Oliveira (2004), and on one side in $10 \%$ - this structure was present in both EEB specimens on both sides. In O. rufus, the alisphenoid strut is less frequent, being present in less than $50 \%$ of the specimens, and in O. quaestor it is absent (Gonçalves \& Oliveira, 2004).

Field observations: Two males were collected during the expeditions at EEB, both with pitfall traps in 2004: one adult in January and one juvenile in May. Observations on their reproductive status were not assessed in the field.

\section{Thaptomys nigrita (Lichtenstein 1829)}

Voucher material: $\mathrm{n}=12$; female: uncatalogued: EEB $613,777,803,832,880,1017$; male: MZUSP 33711, 33712; uncatalogued: EEB 721, 791, 821, 1020.

Specimens identification: Small size (see body measurements on Table 17). Dorsal pelage dense and soft, dark brown slightly reddish or copperish colored; dorsal guard hairs long (length ranging from 7.5 to $8 \mathrm{~mm}$ ), with the basal half narrower, gray in color, and the apical half wider, dark brownish; dorsal cover hairs from medium to long (length ranging from 6 to $8 \mathrm{~mm}$ ), grayish colored on basal portion ( $2 / 3)$ and the apical third composed by three bands of color, an inferior band dark gay, a subterminal band copper, and an apical band dark gray; dorsal wool hairs from short to medium (length ranging from 5.5 to $7 \mathrm{~mm}$ ), gray colored with copperish brown apex. Lateral pelage copperish dark brown, sometimes slightly grayish, in general very similar to dorsum; limit between lateral and ventral color very subtle or indistinct. Ventral 


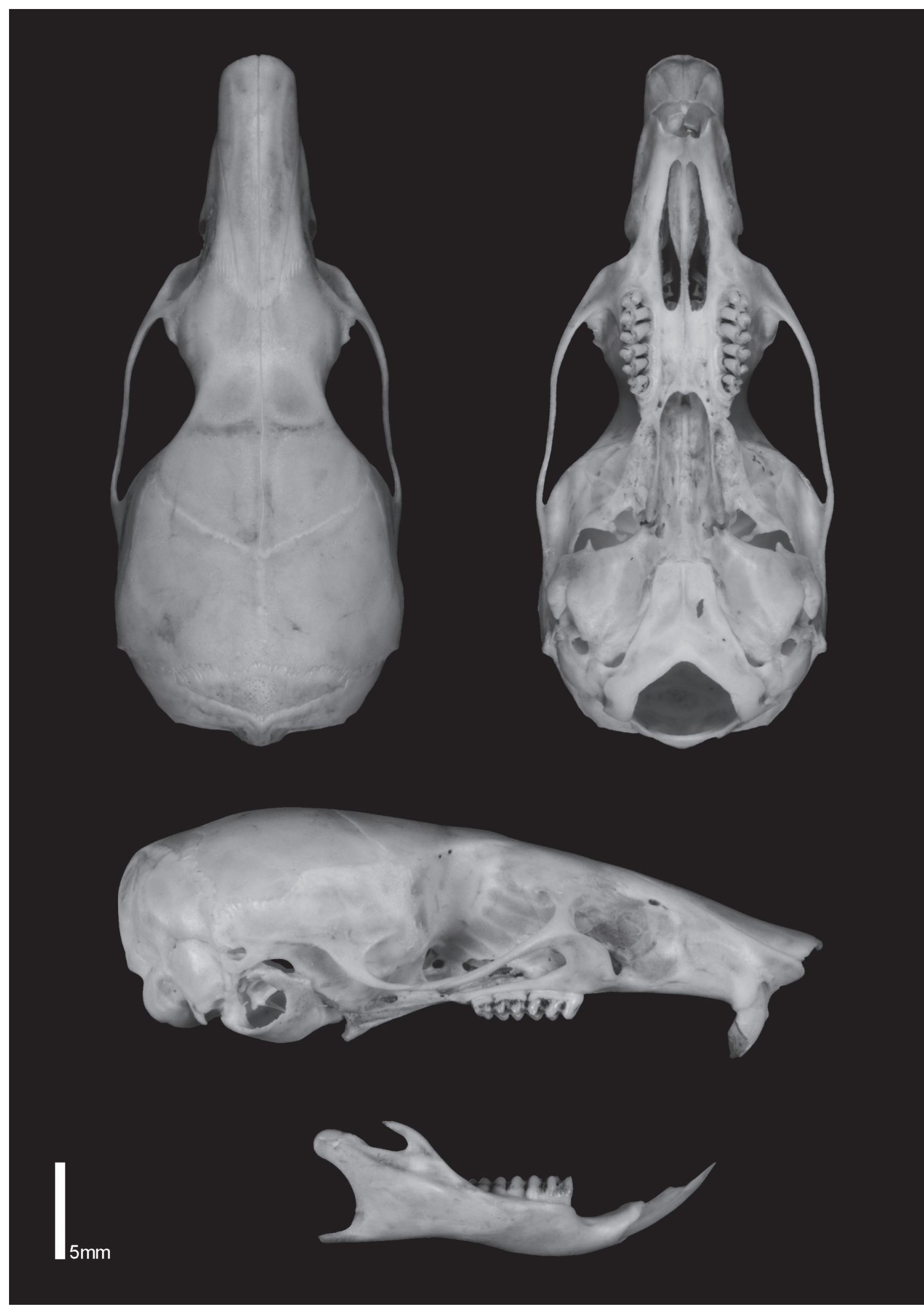

Figure 29. Skull and mandible of Oxymycterus dasytrichus (EEB 850; male; GSL=36.17 mm). Skull on dorsal, ventral, and lateral view, and mandible on lateral view. 
Table 17. Descriptive statistics (mean \pm standard deviation, range, and sample size) of body and cranio-dental measurements (mm), and weight ( $\mathrm{g}$ ), of Thaptomys nigrita from EEB. Values are presented separated for age and sex.

\begin{tabular}{|c|c|c|c|c|}
\hline & \multicolumn{2}{|c|}{ Young and subadult specimens } & \multicolumn{2}{|c|}{ Adult specimens } \\
\hline & Males & Females & Males & Females \\
\hline HBL & 78.00 & 90.0 & $94.67 \pm 5.03(90.0-100.0) 3$ & $(95.0-97.0) 2$ \\
\hline $\mathrm{TL}$ & 53.00 & 47.0 & $46.83 \pm 2.57(44.0-49.0) 3$ & $(48.0-49.0) 2$ \\
\hline HF1 & 18.00 & 18.0 & $17.17 \pm 1.26(16.0-18.5) 3$ & $(16.0-19.0) 2$ \\
\hline HF2 & 21.50 & 20.0 & $18.67 \pm 1.15(18.0-20.0) 3$ & $(18.0-21.5) 2$ \\
\hline EL & 11.00 & 9.50 & $12.17 \pm 1.26(11.0-13.5) 3$ & $(12.0-14.0) 2$ \\
\hline w & 11.00 & 13.50 & $22.17 \pm 2.75(19.0-24.0) 3$ & $(16.5-21.0) 2$ \\
\hline GSL & $(22.24-22.61) 2$ & 22.61 & $23.55 \pm 0.55(23.06-24.14) 3$ & $23.46 \pm 0.51(22.87-23.81) 3$ \\
\hline CIL & $(20.99-21.98) 2$ & $(20.00-21.30) 2$ & $22.71 \pm 0.44(22.36-23.33) 4$ & $22.72 \pm 0.66(21.96-23.15) 3$ \\
\hline CZL & $(15.78-16.63) 2$ & $(14.89-16.13) 2$ & $17.04 \pm 0.37(16.59-17.46) 4$ & $17.02 \pm 0.46(16.49-17.34) 3$ \\
\hline LD & $(6.15-6.65) 2$ & $(6.15-6.16) 2$ & $6.91 \pm 0.26(6.58-7.20) 4$ & $6.91 \pm 0.34(6.52-7.12) 3$ \\
\hline LIF & $(4.55-5.06) 2$ & $(4.34-4.66) 2$ & $5.03 \pm 0.17(4.85-5.25) 4$ & $5.01 \pm 0.09(1.39-1.57) 3$ \\
\hline BIF & $(1.43-1.65) 2$ & $(1.52-1.52) 2$ & $1.57 \pm 0.12(1.43-1.69) 4$ & $1.49 \pm 0.09(1.39-1.57) 3$ \\
\hline LM & $(3.58-3.80) 2$ & $(3.37-3.54) 2$ & $3.51 \pm 0.07(3.42-3.58) 4$ & $3.61 \pm 0.14(3.49-3.76) 3$ \\
\hline BM1 & $(0.94-1.06) 2$ & $(1.00-1.06) 2$ & $0.98 \pm 0.05(0.92-1.03) 4$ & $1.03 \pm 0.03(0.99-1.05) 3$ \\
\hline ID & $(1.03-1.08) 2$ & $(1.02-1.11) 2$ & $1.13 \pm 0.02(1.11-1.15) 4$ & $1.18 \pm 0.04(1.13-1.21) 3$ \\
\hline IW & $(1.27-134) 2$ & $(1.26-1.29) 2$ & $1.38 \pm 0.03(1.35-1.41) 4$ & $1.54 \pm 0.11(1.42-1.62) 3$ \\
\hline LN & $(8.26-8.55) 2$ & 8.09 & $8.63 \pm 0.51(8.15-9.16) 3$ & $8.75 \pm 0.47(8.40-9.28) 3$ \\
\hline BR & $(3.29-3.55) 2$ & $(3.30-3.33) 2$ & $3.32 \pm 0.10(3.20-3.44) 4$ & $3.38 \pm 0.13(3.25-3.51) 3$ \\
\hline RL & $(3.29-3.37) 2$ & 7.62 & $7.97 \pm 0.16(7.80-8.11) 3$ & $8.14 \pm 0.26(7.84-8.33) 3$ \\
\hline ZB & $(11.94-11.99) 2$ & $(11.39-12.32) 2$ & $12.84 \pm 0.28(12.46-13.11) 4$ & $12.93 \pm 0.66(12.27-13.58) 3$ \\
\hline BZP & $(2.03-2.24) 2$ & $(1.93-2.04) 2$ & $2.19 \pm 0.17(2.05-2.44) 4$ & $2.32 \pm 0.18(2.20-2.52) 3$ \\
\hline LIB & $(4.82-5.03) 2$ & $(4.82-4.87) 2$ & $4.94 \pm 0.12(4.79-5.06) 4$ & $4.87 \pm 0.15(4.77-5.04) 3$ \\
\hline OFL & $(7.88-8.13) 2$ & $(7.45-7.72) 2$ & $8.01 \pm 0.20(7.80-8.28) 4$ & $8.17 \pm 0.35(7.80-8.49) 3$ \\
\hline PL & $(9.53-10.24) 2$ & $(9.26-9.68) 2$ & $10.41 \pm 0.13(10.24-10.53) 4$ & $10.42 \pm 0.22(10.18-10.62) 3$ \\
\hline LPB & $(3.05-3.14) 2$ & $(2.97-3.65) 2$ & $3.41 \pm 0.33(3.08-3.85) 4$ & $3.41 \pm 0.04(3.38-3.46) 3$ \\
\hline BBP & $(2.51-2.51) 2$ & $(2.30-2.31) 2$ & $2.53 \pm 0.20(2.38-2.81) 4$ & $2.68 \pm 0.23(2.44-2.89) 3$ \\
\hline HBC & $(7.32-7.43) 2$ & $(7.15-7.16) 2$ & $7.78 \pm 0.11(7.69-7.93) 4$ & $7.51 \pm 0.18(7.38-7.71) 3$ \\
\hline BBC & $(10.62-11.24) 2$ & $(10.87-11.11) 2$ & $11.53 \pm 0.32(11.12-11.82) 4$ & $11.22 \pm 0.34(11.35-11.83) 3$ \\
\hline IPB & $(5.45-5.55) 2$ & $(6.47-6.56) 2$ & $6.63 \pm 0.65(6.18-7.59) 4$ & $5.34 \pm 0.57(4.72-5.84) 3$ \\
\hline IPL & $(1.00-1.27) 2$ & $(1.07-1.39) 2$ & $1.26 \pm 0.14(1.12-1.45) 4$ & $0.88 \pm 0.19(0.69-1.07) 3$ \\
\hline ОСB & $(5.89-5.97) 2$ & $(5.47-6.17) 2$ & $6.04 \pm 0.14(5.88-6.19) 4$ & $6.08 \pm 0.05(6.03-6.12) 3$ \\
\hline
\end{tabular}

pelage color ranging from grayish copper brown to grayish light brown; individual ventral hairs with basal portion (3/4) gray colored and apical portion (1/4) copper brown or light brown colored. Mystacial vibrissae short, not reaching the base of the pinnae when laid back. Pinnae small and rounded; covered internally with numerous hairs, brown colored on the basal portion of pinnae and copper on the apical portion, and with less numerous hairs externally, grayish colored. Dorsal surface of manus covered by dark brown hairs; digits with medium to long claws (claw of dlll with length ranging from 2.3 to $3 \mathrm{~mm} ; \mathrm{n}=3$ ). Pes short and narrow, dorsally covered by dark brown hairs; dIII longer than dII and shorter than dIV; digits with medium to long claws (claw of dIII with length ranging from 2.4 to $3.1 \mathrm{~mm} ; \mathrm{n}=3$ ); tufts of ungual hairs sparse and moderately short, a few hairs extending beyond the claws, brown colored. Tail shorter than head and body $(50.2 \% \pm 1.4$ of head and body length, ranging from 48.9 to $52.2 \%$; $n=5$ ); covered by short hairs, with length of one and a half to two scale at the basal portion on both surfaces; scales arranged in 27 to 32 transverse rows per centimeter at the base of the tail and between 29 and 34 rows on the apical centimeter of the tail $(n=3)$; dorsal tail color dark brown, with dark brown hairs, and ventral tail color brown slightly lighter especially at the base, with whitish and brown hairs.

Skull short and wide (Fig. 30; Table 17). Rostrum short. Nasals anteriorly short, not extending beyond the level of superior incisors, and posteriorly long, extending beyond the maxillary-frontal-lacrimal suture; with posterior margin sharp $(81.8 \%)$ or rounded $(18.2 \%)$ $(n=11)$. Premaxillae posteriorly short, ending anteriorly to nasals. Lacrimal with equal contact with maxillary and frontal $(80 \%)$ or in contact mainly with maxillary (20\%) $(\mathrm{n}=10)$. Zygomatic notch shallow. Interorbital region broad, hourglass in shape (72.8\%) or slightly posteriorly convergent (27.2\%), with rounded supraorbital margins $(n=11)$. Frontosquamosal suture not collinear with frontoparietal suture. Braincase large, broad and inflated, without temporal crests. Parietal entirely restricted to the dorsal surface of the braincase (9\%), partially expanded to the lateral surface of the braincase (45.5\%), or largely expanded onto lateral surface of the braincase $(45.5 \%)(n=11)$. Zygomatic plate narrow with anterodorsal margin rounded and conspicuously anterior to superior maxillary root of zygoma; posterior margin 


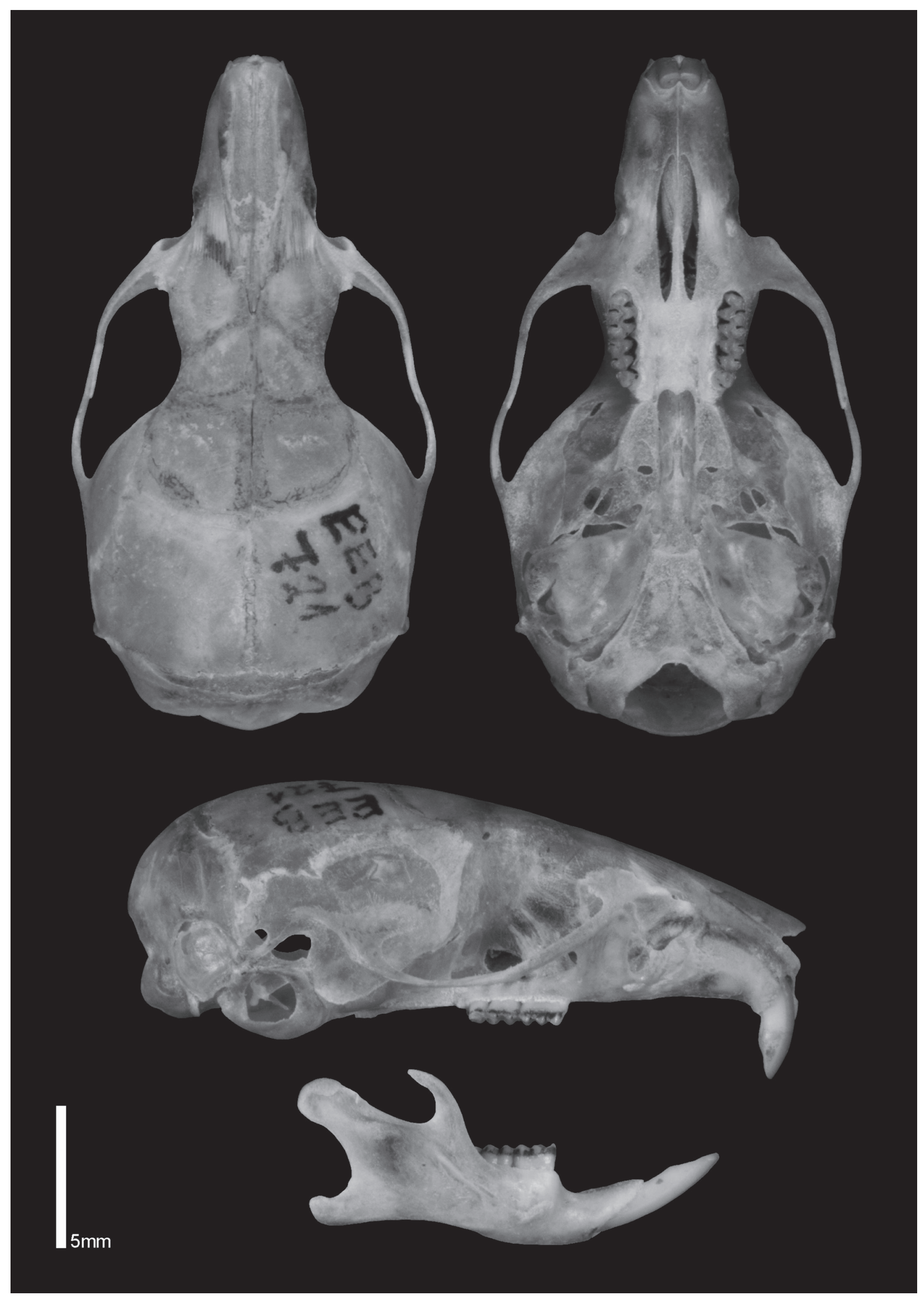

Figure 30. Skull and mandible of Thaptomys nigrita (EEB 721; male; GSL = $24.14 \mathrm{~mm}$ ). Skull on dorsal, ventral, and lateral view, and mandible on lateral view. 
of zygomatic plate situated approximately even with the alveolus of M1 (90.9\%) or situated anterior to the alveolus of M1 $(9.1 \%)(n=11)$. Jugal present and large, maxillary and squamosal processes of zygomatic arch not overlapping. Anterior opening of alisphenoid canal covered by the alisphenoid strut. Alisphenoid strut very broad and robust, buccinator-masticatory foramen and accessory oval foramen separate; buccinator-masticatory foramen laterally covered by the alisphenoid strut. Posterolateral border of the parapterygoid plate absent (77.8\%), setting a large foramen formed by the posterior opening of alisphenoid canal, oval foramen and accessory oval foramen, or present but very narrow and delicate (22.2\%), separating the posterior opening of alisphenoid canal and the accessory oval foramen $(n=9)$. Squamosal-alisphenoid groove present on braincase lateral view, in the squamosal and alisphenoid bones; sphenofrontal foramen present; posterior opening of alisphenoid canal large; stapedial foramen present and large in the ectotympanic bullae, on the petrosal-ectotympanic suture (stapedial and carotid circulatory pattern 1; Voss, 1988). Subsquamosal fenestra present. Mastoid with conspicuous fenestra. Incisive foramina narrow and long, with slightly convex edges; posterior margin surpassing the anterior face of $\mathrm{M} 1$ and reaching the anterocone $(27.3 \%)$, reaching the protoflexus $(63.6 \%)$, or reaching the protocone $(9.1 \%)(n=11)$. Palate short and wide, with the central region deeper and lateral excavations shallow; posterolateral palatal pits absent (18.2\%), present as one small pit on each side of posterior palate $(72.7 \%)$, or present as one single opening on the left side and two foramina on the right side (9.1\%) $(n=11)$. Mesopterygoid fossa not reaching the posterior face of $M 3$ (27.3\%), reaching the posterior face of $M 3$ (54.5\%), or surpassing the posterior face of M3 (18.2\%) ( $n=11$ ); sphenopalatine vacuities absent, mesopterygoid roof completely ossified. Parapterygoid plate with similar width to the middle portion of mesopterygoid fossae $(80 \%)$, or narrower than the middle portion of mesopterygoid fossae $(20 \%)(n=10)$. Auditory bullae small, exposed flange of periotic extends to internal carotid canal $(27.3 \%)$, or intermediate, exposed wedge of periotic smaller and not contributing to wall of carotid canal $(72.7 \%)(n=11)$. Mandible elongated, with ramus moderately high (Fig. 30). Superior and posterior notches deep. Capsular process of lower incisor alveolus present but reduced as a slight rounded elevation (27.3\%), or present and well developed $(72.7 \%)(n=11)$. Superior and inferior masseteric ridges very subtle and converge anteriorly in an opening "V" shaped. Upper incisors orthodont. Maxillary toothrows from parallels to slightly anteriorly divergent. M1 with anterocone divided into labial and lingual conules by the anteromedian flexus; anteroloph present but reduced as a projection of anterolabial conule; mesoloph present but reduced as a projection of paracone toward to metacone. $\mathrm{m} 1$ with anteroconid divided into labial and lingual conulids by the anteromedian flexid; anterolophid absent; protostylid usually present; mesolophid present but reduced as a projection of metaconid; ectostylid usually present.
Field observations: Twelve specimens of $T$. nigrita were captured at EEB, 10 with pitfall traps and two with conventional traps. In the expedition of December, 2003, seven specimens were with information on the reproductive activity for some specimens (males with scrotal testes and females with open vaginas) and also on reproductively inactive specimens. In 2004, one specimen was captured in April, one in May and one in December. In 2011 two specimens were collected in December. Observations on the reproductive state of the specimens trapped in 2004 and 2011 were not recorded.

\section{Tribe Oryzomyini \\ Drymoreomys albimaculatus Percequillo, Weksler \& Costa 2011}

Voucher material: $\mathrm{n}=2$; female: MZUSP 33782; male: uncatalogued: EEB 924.

Specimens identification: Both specimens captured at the EEB exhibit thin and wavy grayish pelage, with dominance of wool hairs, as well as weakly worn molars, features typical of young individuals. Therefore, some external and cranial information presented here is derived from the original description of the genus and species (Percequillo et al., 2011), with new amendments based on the specimens from EEB.

Medium size (see body measurements on Table 18). Dorsal fur very long, extremely lax and dense; pelage color ochraceous brown or orange, grizzled with brownish, with some reddish brown hairs; guard hairs very long and moderately stiff (length ranging from 17 to $21 \mathrm{~mm}$ ), with half proximal portion grayish and distal portion reddish to dark brown; cover hairs long (length ranging from 14 to $21 \mathrm{~mm}$ ), with distal portion banded, with basal and terminal bands dark brown and subterminal band orangish; wool hairs shorter, wavy and thin (length ranging from 12 to $14 \mathrm{~mm}$ ), with basal and very short terminal $(1 / 10$ of hair length) bands dark brown and subterminal band orangish. Lateral pelage predominantly reddish or rusty brown; transition with ventral region sharply delimited. Ventral pelage predominantly grayish, with gray-based white hairs; ventral pelage with distinct patches of self-colored white hairs, in gular, thoracic and, eventually, inguinal positions. Mystacial vibrissae very long and dense, surpassing pinnae when laid backwards. Pinnae small and rounded, covered internally by short golden hairs and densely covered externally by orangish to reddish brown short hairs. Manus covered dorsally by short brown hairs; digits II to V (dl reduced) covered by whitish/grayish hairs, not developed in conspicuous ungual tufts; digits with short claws (claw of dIII = $1.7 \mathrm{~mm}$; $n=1)$. Pes short and moderately wide, with proximal dorsal surface covered by short brown hairs, forming dark patches of variable size, and with dorsal distal surface and digits covered by grayish hairs; dIII longer than dll and equal in length to dIV; claw moderate in size (claw of dIII = $2.85 \mathrm{~mm} ; \mathrm{n}=1$ ); digits with conspicuous grayish to whitish ungual tufts, long and dense exceeding 
Table 18. Body and cranio-dental morphometric values (mm), and weight $(\mathrm{g})$ of Drymoreomys albimaculatus from localities in São Paulo State. *Adult specimens measured by Percequillo et al. (2011). ${ }^{* *}$ Young specimens from EEB.

\begin{tabular}{|c|c|c|c|c|c|c|c|c|c|c|}
\hline & \multicolumn{4}{|c|}{ Females } & \multicolumn{2}{|c|}{ Males } & \multicolumn{4}{|c|}{ Sex unknown } \\
\hline & MZUSP 34716* & MAM $15^{*}$ & MVZ 182089* & MZUSP $33782^{* *}$ & RG 47* & EEB 924** & AB 397* & AB 423* & B0 24* & B0 41* \\
\hline HBL & 149 & 135 & 125 & 105 & 103 & - & 109 & 143 & - & - \\
\hline TL & 176 & 170 & 165 & 145 & 113 & - & 150 & 162 & - & - \\
\hline HF2 & 28 & 30 & 30 & 25 & 23 & - & 18.5 & 19 & - & - \\
\hline EL & 20 & 20 & 20 & 16 & 18 & - & 27.5 & 29 & - & - \\
\hline W & 57 & 58 & 50 & 32 & 21 & - & 52 & 102 & - & - \\
\hline CIL & 30.67 & 30.79 & 30.53 & 26.66 & 24.94 & 25.83 & 28.22 & 29.92 & 28.26 & 29.93 \\
\hline LD & 8.56 & 8.37 & 8.13 & 7.29 & 6.87 & 7.47 & 7.99 & 8.24 & 7.88 & 7.98 \\
\hline LIF & 6.33 & 6.37 & 5.82 & 5.56 & 5.16 & 5.36 & 5.59 & 5.99 & 5.74 & 5.78 \\
\hline BIF & 2.82 & 2.85 & 2.65 & 2.56 & 2.30 & 2.71 & 2.55 & 3.03 & 2.79 & 2.96 \\
\hline LM & 5.25 & 5.43 & 5.01 & 5.19 & 5.03 & 4.93 & 5.00 & 5.15 & 5.02 & 5.07 \\
\hline BM1 & 1.49 & 1.48 & 1.44 & 1.45 & 1.37 & 1.45 & 1.38 & 1.39 & 1.42 & 1.44 \\
\hline LN & 12.37 & 12.26 & 12.52 & 10.67 & 10.44 & 10.84 & 11.57 & 12.06 & - & - \\
\hline BR & 5.99 & 5.95 & 5.31 & 4.74 & 4.77 & 4.06 & 4.82 & 5.73 & 5.33 & 5.57 \\
\hline ZB & 17.84 & 16.66 & 17.08 & 14.91 & 14.39 & 14.48 & 15.45 & 17.60 & - & - \\
\hline BZP & 2.65 & 2.43 & 2.65 & 1.92 & 1.90 & 2.31 & 2.46 & 2.55 & 2.15 & 2.47 \\
\hline LIB & 5.69 & 5.64 & 5.33 & 5.26 & 5.40 & 5.07 & 5.01 & 5.57 & 5.32 & 5.52 \\
\hline OFL & 11.37 & 11.24 & 11.32 & 10.57 & 9.96 & 10.04 & 10.69 & 11.25 & 10.60 & 11.66 \\
\hline LPB & 5.33 & 5.38 & 5.59 & 4.91 & 4.70 & 4.88 & 5.37 & 5.77 & 5.07 & 5.34 \\
\hline BBP & 3.07 & 2.83 & 2.94 & 2.44 & 2.41 & 2.81 & 2.67 & 2.75 & 2.95 & 2.96 \\
\hline
\end{tabular}

the length of claws; pes with fleshy and very large interdigital pads; thenar and hypothenar pads large; plantar integument between pads with squamae; pads flesh colored. Tail longer than head and body (ca. 138\% of head and body length; $n=1$ ); tail densely covered by short hairs, whose length equals the length of two scale rows, both on dorsal and ventral surface; apical tuft of hairs absent; scales very small and arranged in 29 transverse rows per centimeter at the base of the tail, in 34 rows on the central portion of the tail, and in 23 rows on the tip of the tail ( $n=1)$; tail brownish (skin and hairs), dorsally and ventrally.

Skull robust and elongated (Fig. 31; Table 18). Rostrum moderately long; short rostral tube present, formed by the anterior portions of nasals and premaxillae. Nasals long, with posterior margin (nasofrontal suture) surpassing the maxillar-frontal-lacrimal sutures. Premaxillae short posteriorly, ending anteriorly to the nasals. Lacrimal equally contacts maxillar and frontal. Zygomatic notch very shallow and narrow, barely present. Interorbital region slightly converging anteriorly, with supraorbital margins square and sharp, continuous with the sharp temporal margin. Frontosquamosal suture collinear with the frontoparietal suture. Zygomatic arches projected laterally, wider at the squamosal root. Braincase large, with rounded profile. Parietals expanded ventrally, on the lateral surface of braincase. Interparietal long and wide. Rostrum with gnathic process short; rostral tube short. Zygomatic plate very narrow, with anterior margin straight and without free dorsal margin. Zygomatic arches narrow, with jugal large and with maxillar and squamosal processes not overlapping. Stapedial and sphenofrontal foramina, and squamosal-alisphenoid groove absent; large opening for the alisphenoid canal; presence of the anastomotic canal on the surface of alisphenoid (derived carotid and stapedial circulatory pattern 3; Voss, 1988). Alisphenoid strut present, configuring distinct oval accessory and buccinator-masticatory foramina (on both sides or only on the left side of skull). Postglenoid foramen present. Subsquamosal fenestra present. Stapedial and dorsostapedial processes of ectotympanic present; external auditory meatus small and rounded; mastoid with fenestra. Incisive foramina teardrop shaped, moderately wide and long, not reaching the anterior alveoli of M1. Posterior margin of zygomatic plate positioned anteriorly to the alveoli of M1. Palate wide and moderately short, with central region deeper and with two deeper parallel depressions aligned with posterior palatine foramina (configuring a medial crest); palate with posterolateral palatal pits small and situated on the palate level. Mesopterygoid fossa penetrating between molar series, reaching the hypocone of $M 3$; roof of mestopterygoid fossa perforated by large sphenopalatine vacuities, along the presphenoid and basisphenoid. Parapterygoid fossa narrower or with the same width of the mesopterygoid fossa. Auditory bullae small and moderately globose, with the periotic margin extending or not to the internal carotid canal. Mandible delicate, with ramus low (Fig. 31). Coronoid process high and falciform, slightly higher that condyloid process; angular process rounded, extending posteriorly to the condyloid process; superior notch moderately deep and inferior notch moderately shallow. Capsular process of lower incisor absent or present, but small. Superior and inferior masseteric crests weakly developed and converging anteriorly in a V-shape. Upper incisors opisthodont. Upper molar series parallel; molars with main cusps arranged in slightly alternate pairs, with lingual margins anterior to labial ones; labial and lingual flexus slightly interpenetrating on medial plane of molar. M1 with procingulum divided in two conules, anterolabial and anterolingual, by the anteromedian flexus; anterolingual conule slight- 


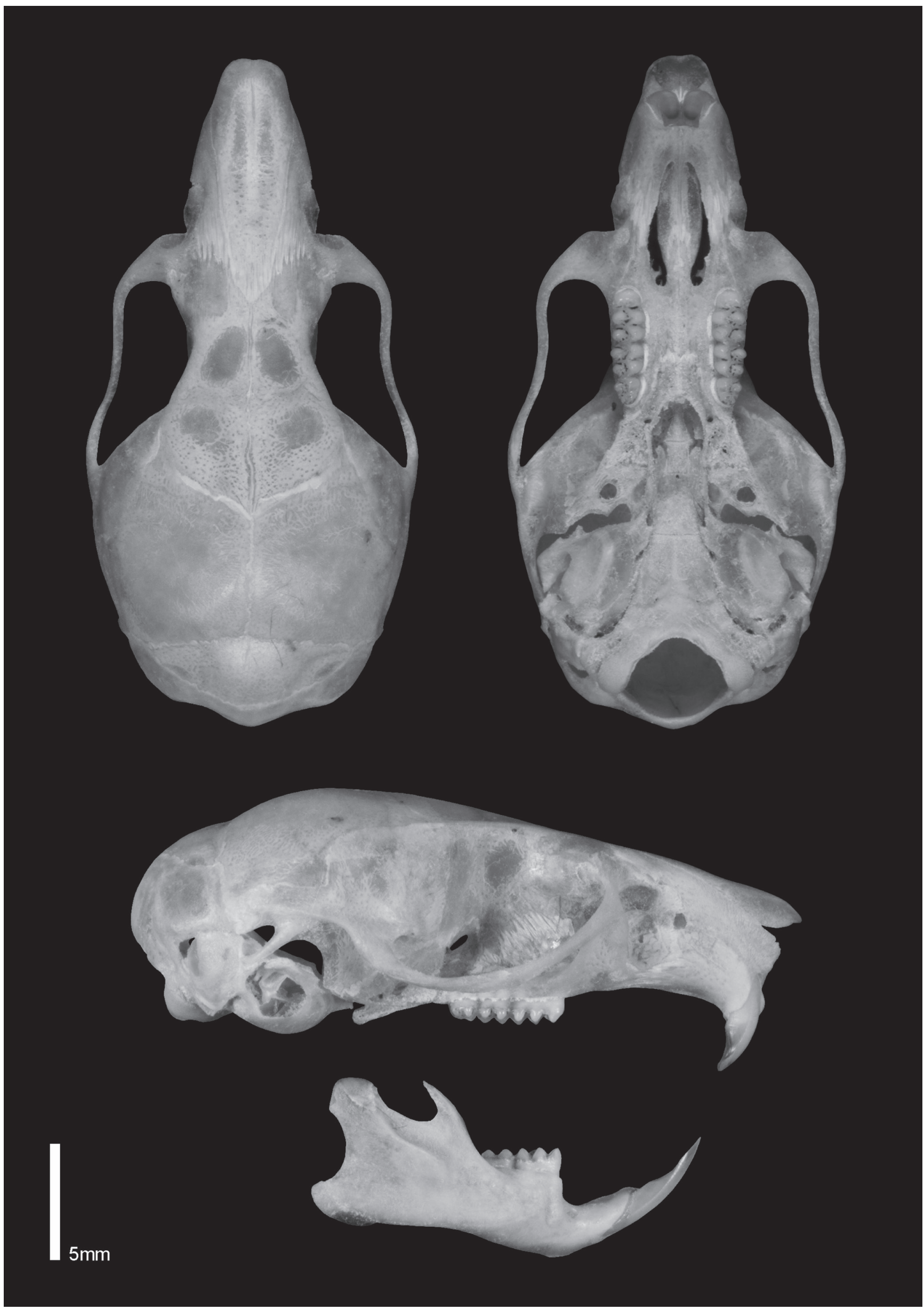

Figure 31. Skull and mandible of Drymoreomys albimaculatus (EEB 924; male; GSL=29.13 mm). Skull on dorsal, ventral, and lateral view, and mandible on lateral view. 
ly larger than anterolabial; anteroloph well developed, reaching the labial margin and labial cingulum; anteroflexus present; mesoloph present, reaching the labial cingulum, fused to the mesostyle; protostyle and enterostyle present. $\mathrm{m} 1$ with procingulum not divided by the anteromedian flexid; anterolophid well developed, reaching the cingulid; mesolophid well developed, fused to mesostylid; protostylid and ectostylid well developed; ectolophid present (with moderate wear).

Remarks: Percequillo et al. (2011) described the genus Drymoeromys and its single species, D. albimaculatus. This species can be easily diagnosed from the remaining AF oryzomyines by a gular patch of self-colored white hairs and a unique set of cranio-dental characteristics, including the presence of a short rostral tube (see Percequillo et al., 2011). One of the specimens from EEB (MZUSP 33782) is a paratype of D. albimaculatus.

Field observations: The specimen MZUSP 33782 is a female with unperforated vagina, captured in the beginning of the summer (December, 2003). The specimen EEB 924 is a juvenile male, with abdominal testes, captured in December, 2004. Both were captured by pitfall traps, in trails next to the main building of the EEB. According to field notes, MZUSP 33782 was captured in a camping site that had been abandoned approximately 10-15 years before this small mammal sampling, and which was covered by a low and unstructured forest with vegetation about 8-10 $\mathrm{m}$ in height.

\section{Euryoryzomys russatus (Wagner 1848)}

Voucher material: $\mathrm{n}=3$; female: uncatalogued: $E E B$ 1001, 1022; male: uncatalogued: EEB 1003.

Specimens identification: Medium size (see body measurements on Table 19). All specimens captured at EEB have the dorsal pelage distinct from what has been described for the species (Musser et al., 1998; Percequillo, 1998, 2015a), as they represent young and subadult specimens, with a shorter and harsher pelage, formed

Table 19. Descriptive statistics (mean \pm standard deviation, range, and sample size) of body measurements $(\mathrm{mm})$, and weight $(\mathrm{g})$ of Euryoryzomys russatus, obtained in Percequillo (1998), and values of EEB specimens.

\begin{tabular}{lcccc}
\hline & Species values & $\begin{array}{c}\text { EEB 1001 } \\
\text { (adult, female) }\end{array}$ & $\begin{array}{c}\text { EEB 1022 } \\
\text { (young, female) }\end{array}$ & $\begin{array}{c}\text { EEB 1003 } \\
\text { (subadult, male) }\end{array}$ \\
\hline HBL & $\begin{array}{c}142.70 \pm 12.05 \\
(105-196) 200\end{array}$ & 150 & 106 & 120 \\
TL & $\begin{array}{c}150.56 \pm 14.50 \\
(90-180) 191\end{array}$ & 151 & 115 & 115 \\
HF1 & $\begin{array}{c}32.93 \pm 1.62 \\
(30-36.5)\end{array}$ & 34 & 29 & 33 \\
HF2 & $\begin{array}{c}35.06 \pm 2.04 \\
(30-40) 200\end{array}$ & 37 & 31 & 35 \\
EL & $\begin{array}{c}23.06 \pm 2.12 \\
(16-29) 195\end{array}$ & 23 & 20 & 20 \\
W & $\begin{array}{c}81.78 \pm 19.05 \\
(40-150) 142\end{array}$ & 106 & 37.5 & 46 \\
\hline
\end{tabular}

by brownish hairs, moderately grizzled with grayish and orangish hairs; typical adult E. russatus exhibit a long, soft and moderately dense pelage, reddish orange intensely grizzled with dark brown, resulting in a ferruginous dorsal coloration (op. cit.).

Dorsal pelage with guard hairs long and moderately stiff (length ranging from 13 to $15 \mathrm{~mm}$ ), with half proximal portion grayish and distal portion dark brown, almost black, and tip golden; cover hairs moderately long (length ranging from 10 to $12 \mathrm{~mm}$ ), with proximal two thirds grayish and distal third portion banded, with basal and terminal bands dark brown and subterminal band orangish; wool hairs shorter, wavy and thin (length ranging from 7 to $8 \mathrm{~mm}$ ), with the apical portion banded, with basal and terminal bands dark brown and subterminal band orangish. Lateral pelage predominantly orange-reddish or rusty brown; transition with ventral region sharply delimited. Ventral pelage predominantly grayish or grayish-white, with gray-based (2/3) white hairs. Mystacial vibrissae long and moderately dense, not surpassing pinnae when laid backwards. Pinnae large and rounded, covered internally by short brown and orangish hairs and densely covered externally by short brown hairs. Manus covered dorsally by predominant short white hairs and few brown based hairs; digits with short claws (claw of dll with 1.3 to $1.4 \mathrm{~mm} ; \mathrm{n}=2$ ); digits without conspicuous ungual tufts. Pes long and narrow, with dorsal surface covered by short white hairs; with dIII longer than other digits; claw moderate in size (claw of dIII with 2.45 to $3.1 \mathrm{~mm} ; \mathrm{n}=2$ ); digits with conspicuous whitish ungual tufts, very long and very dense exceeding the length of claws, concealing them; pes with small interdigital pads; thenar and hypothenar pads present; plantar integument between pads without squamae, smooth; pads dark brown, darker to much darker than the remaining ventral surface. Tail similar to head and body length $(99.8 \% \pm 7.6$ of head and body length, ranging from 95 to $108.5 \% ; n=3)$; tail covered by short hairs, whose length equals the length of two scale rows on dorsal surface and of less than two scale rows on the ventral surface; apical tuft of hairs absent; scales very small; scales arranged in 18 to 25 transverse rows per centimeter at the base of the tail, between 17 to 23 rows per $\mathrm{cm}$ on the central portion of the tail, and between 23 and 27 rows on the apical centimeter of the tail $(n=2)$; tail brownish (skin and hairs) dorsally, and ventrally whitish (proximal half) and brownish (distal half); tail bicolored on proximal half.

Skull robust and elongated (Fig. 32; Table 20). Rostrum moderately long and narrow; rostral tube absent. Nasals long, expanded laterally distally, with posterior margin (nasofrontal suture) surpassing the maxillar-frontal-lacrimal sutures. Nasofrontal suture triangular or rounded. Premaxillae projected posteriorly, aligned to the nasals or surpassing them. Lacrimal equally contacts maxillar and frontal. Zygomatic notch deep and wide. Interorbital region slightly converging anteriorly, with supraorbital margins square or beaded, forming moderately dorsal projections, continuous with the sharp temporal margin. Frontosquamosal suture collinear or not with the frontoparietal suture. Zygomatic arches projected later- 
Table 20. Descriptive statistics (mean \pm standard deviation, range, and sample size) of cranio-dental measurements (mm) of Euryoryzomys russatus, obtained in Abreu-Júnior et al. (2012), and values of EEB specimens.

\begin{tabular}{|c|c|c|c|c|c|}
\hline & \multicolumn{3}{|c|}{ Females } & \multicolumn{2}{|c|}{ Males } \\
\hline & Species values & EEB 1001 (adult) & EEB 1022 (young) & Species values & EEB 1003 (subadult) \\
\hline GSL & $35.11 \pm 1.30(31.94-38.16) 101$ & 35.41 & 28.50 & $35.45 \pm 1.43(30.70-39.22) 159$ & 30.24 \\
\hline CIL & $31.34 \pm 1.19(28.45-34.65) 103$ & 31.59 & 25.42 & $31.77 \pm 1.33(27.99-35.54) 159$ & 26.52 \\
\hline LD & $8.88 \pm 0.56(5.49-10.22) 113$ & 8.81 & 6.94 & $9.08 \pm 0.51(7.66-10.48) 164$ & 7.30 \\
\hline LM & $5.11 \pm 0.18(4.56-5.49) 111$ & 5.32 & 5.05 & $5.10 \pm 0.17(4.62-5.59) 163$ & 4.81 \\
\hline BM1 & $1.49 \pm 0.05(1.33-1.67) 114$ & 1.54 & 1.48 & $1.49 \pm 0.06(1.23-1.67) 167$ & 1.42 \\
\hline LIF & $5.90 \pm 0.344(5.17-6.90) 115$ & 5.95 & 4.84 & $5.95 \pm 0.35(4.96-6.69) 167$ & 4.94 \\
\hline BIF & $2.32 \pm 0.17(1.89-2.77) 116$ & 2.39 & 2.28 & $2.36 \pm 0.17(1.91-3.00) 167$ & 2.07 \\
\hline IPB & $10.18 \pm 0.60(8.43-12.00) 105$ & 9.45 & 10.30 & $10.28 \pm 0.67(8.32-11.82) 161$ & 9.82 \\
\hline IPL & $4.37 \pm 0.49(3.17-5.20) 104$ & 4.14 & 3.14 & $4.26 \pm 0.50(3.15-5.40) 159$ & 3.54 \\
\hline LN & $14.40 \pm 0.78(12.51-16.54) 111$ & 14.92 & 11.68 & $14.47 \pm 0.85(11.41-16.69) 160$ & 11.81 \\
\hline LPB & $6.65 \pm 0.34(5.81-7.55) 116$ & 6.77 & 5.53 & $6.67 \pm 0.36(5.53-7.90) 165$ & 5.55 \\
\hline LIB & $5.46 \pm 0.25(4.67-6.08) 116$ & 5.35 & 5.01 & $5.54 \pm 0.27(4.81-6.26) 168$ & 5.46 \\
\hline ZB & $17.84 \pm 0.67(15.90-19.59) 99$ & 18.16 & 15.70 & $17.92 \pm 0.77(15.67-19.69) 137$ & 15.99 \\
\hline BZP & $4.07 \pm 0.26(3.30-4.74) 116$ & 4.22 & 2.92 & $4.09 \pm 0.28(3.44-5.01) 167$ & 3.67 \\
\hline CZL & $23.88 \pm 0.89(21.72-26.20) 103$ & 24.36 & 19.57 & $24.25 \pm 0.96(21.48-26.67) 158$ & 20.97 \\
\hline OFL & $11.77 \pm 0.43(10.76-12.87) 116$ & 11.77 & 9.91 & $11.93 \pm 0.46(10.60-12.87) 168$ & 10.49 \\
\hline
\end{tabular}

ally, wider at the squamosal root. Braincase large, with elongated profile. Parietals not expanded ventrally, on the lateral surface of braincase. Interparietal long and wide. Rostrum with gnathic process short; rostral tube absent. Zygomatic plate moderately wide, with anterior margin straight or slightly concave; free dorsal margin projected anteriorly, with rounded anterodorsal margin. Zygomatic arches narrow, with jugal large and with maxillar and squamosal processes not overlapping. Stapedial foramen large; sphenofrontal foramen present and squamosal-alisphenoid groove present; large opening for the alisphenoid canal (primitive carotid and stapedial circulatory pattern 1; Voss, 1988). Alisphenoid strut present, configuring distinct oval accessory and buccinator-masticatory foramina (on both sides [one specimen] or only on the left side of skull [two specimens]). Postglenoid foramen present. Subsquamosal fenestra present. Stapedial process of ectotympanic present; external auditory meatus small and rounded. Mastoid predominantly ossified, with small fenestra on the contact with the exoccipital margin, or with large fenestra (in young specimens). Incisive foramina moderately long and narrow, with lateral margins parallel or slightly wider medially; anterior margins acute and narrow and posterior margins rounded, not reaching the anterior alveoli of $\mathrm{M} 1$. Posterior margin of zygomatic plate positioned anteriorly to the alveoli of M1. Palate wide and long, with central region deeper and with two deeper parallel depressions aligned with posterior palatine foramina (configuring a small medial crest); palate with posterolateral palatal pits small to moderate and situated on the palate level. Mesopterygoid fossa not penetrating between molar series; roof of mestopterygoid fossa perforated by small and narrow sphenopalatine vacuities, along the suture of the presphenoid and basisphenoid. Parapterygoid fossa wider or with the same width of the mesopterygoid fossa. Auditory bullae small and moderately globose, with the periotic margin extending to the internal carotid canal. Mandible robust, with ramus high (Fig. 32). Coronoid process high and triangular or weakly falciform, equal in height to the condyloid process; angular process rounded, on the same level of the condyloid process; superior notch and inferior notch shallow. Capsular process of lower incisor present, small (in young specimens) to large (in adult specimens). Superior and inferior masseteric crests weakly developed (inferior more developed) and converging anteriorly in a V-shape. Upper incisors opisthodont. Upper molar series parallel; molars with main cusps arranged in opposite pairs; labial and lingual flexus slightly interpenetrating on medial plane of molar. M1 with procingulum not divided in two conules; anteroloph well developed, reaching the labial margin and labial cingulum; anteroflexus present; mesoloph present, reaching the labial cingulum, fused to the mesostyle; with wear this flexus will be closed, forming a distinct mesofosset; in few specimens, a small paralophule will bissect the paraflexus in two, transversally, forming a medial parafosset; then, two enamel islands will be present; protostyle and enterostyle present. M2 similar to M1, with parafosset and mesofosset present in most specimens; a few individuals exhibiting a third fosset, a labial parafosset. $\mathrm{m} 1$ with procingulum not divided by the anteromedian flexid; anterolophid well developed, reaching the cingulid; mesolophid well developed, fused to mesostylid; protostylid and ectostylid small; ectolophid present, but in low frequencies (with moderate wear).

Remarks: Percequillo (1998) examined the non-geographic morphological variation of $E$. russatus in some populations of the AF and he documented a seasonal variation in the structure and coloration of the pelage: specimens collected in the warmer months (e.g., from December to April in the sample collected at Estação Biológica de Boracéia) had thin and rough pelage, with new emerging pelage and epidermis dark due to pigment. This condition was found in specimen EEB 1001, which was collected in December, corroborating the seasonality of this feature. 


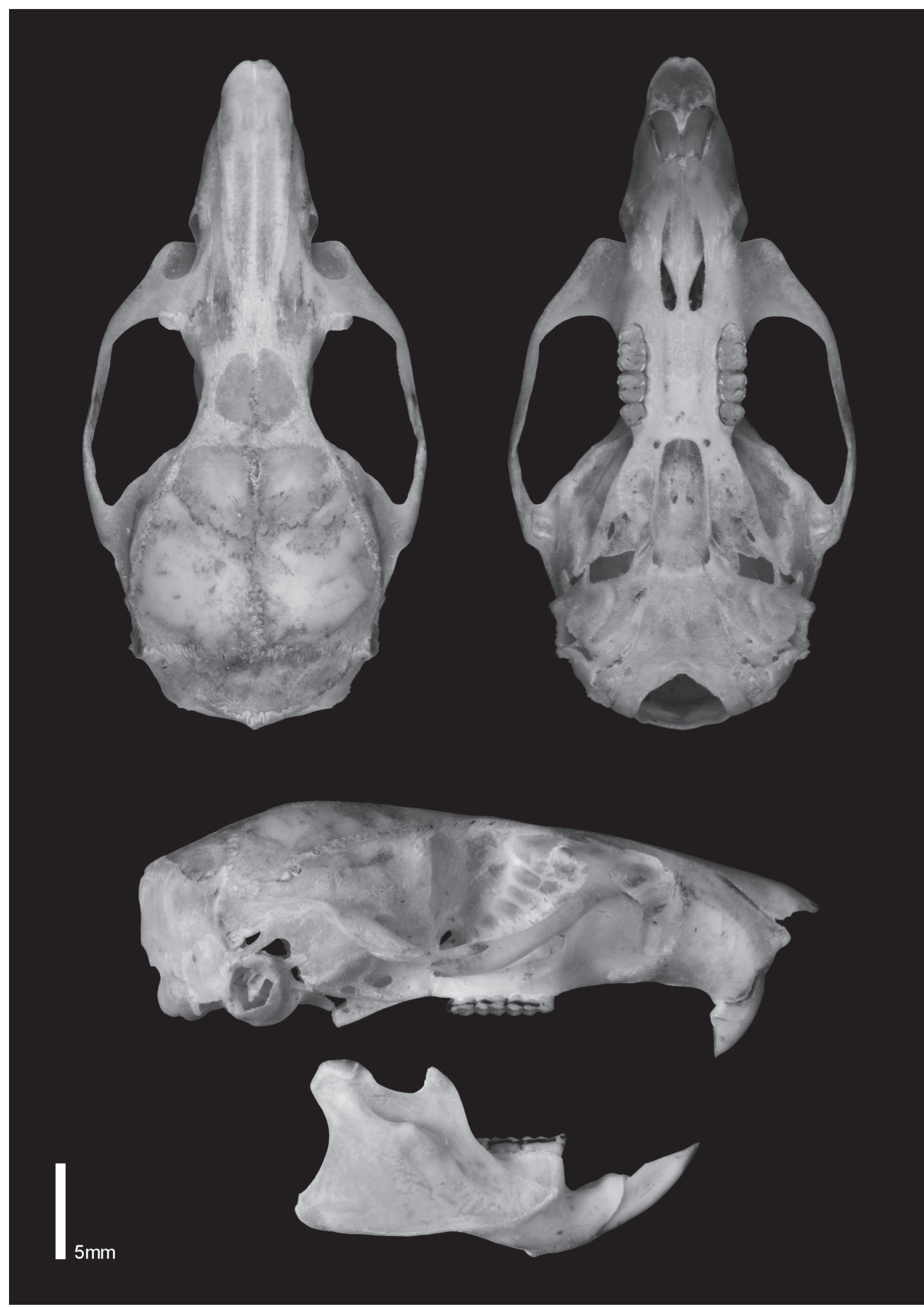

Figure 32. Skull and mandible of Euryoryzomys russatus (EEB 1001; female; $G S L=35.41 \mathrm{~mm}$ ). Skull on dorsal, ventral, and lateral view, and mandible on lateral view. 
Field observations: Specimens of E. russatus were collected at EEB only in the last expedition, in December, 2011. Before that, no individuals of the species had been captured, despite the larger sampling effort performed previously in 2003, 2004 and 2005. The specimens collected at EEB are two females (one adult with mammal glands visible and a juvenile without indication of reproductive activity) and a juvenile male (according to pelage and wear of molars) with scrotal testes. These specimens were captured by Sherman traps along the same trail.

\section{Nectomys squamipes (Brants 1827)}

Voucher material: $\mathrm{n}=2$; female: uncatalogued: EEB 800; sex unknown: uncatalogued:TX080023.

Specimens identification: Very large size. Dorsal pelage short, soft, compact and moderately dense, brown weakly grizzled with golden yellow hairs; guard hairs long and moderately stiff (about $14 \mathrm{~mm}$ in length), with half proximal portion thinner and grayish and distal portion wider and stiffer, and black; cover hairs moderately long (about $12 \mathrm{~mm}$ ), with proximal three quarters grayish and distal quarter portion banded, with basal band orange and terminal band black; wool hairs very numerous, shorter, wavy and thin (about $10 \mathrm{~mm}$ in length), with the apical portion banded, with basal band orange and terminal band black. Lateral pelage denser and laxer than dorsal pelage, predominantly brownish yellow or rusty brown; transition with ventral region subtle, without a sharp limit. Ventral pelage ochraceous brown or ochraceous orange on the abdominal region and grayish cream on the inguinal, thoracic and gular regions; specimens with a distinct patch of self-colored cream hairs on gular region; hairs with gray base $(2 / 3$ or $1 / 2)$ and cream/ochraceous orange or whitish/whitish cream tip. Mystacial vibrissae moderately long and dense, reaching base of pinnae when laid backwards. Pinnae small and rounded covered internally by few short whitish hairs and densely covered externally by short brown hairs. Manus short and moderately wide, covered dorsally by predominant short white hairs and few brown-based hairs; manus with long digits bearing long claws (claw of dIII = $3.8 \mathrm{~mm} ; \mathrm{n}=1$ ); dl vestigial, dll to dV longer, without conspicuous ungual tufts. Pes very long and moderately wide, robust, with dorsal surface covered by short white hairs; digit II similar in size to digit IV and longer than dIV, dIII longer than other digits; digits with long claws (claw of dIII = $6.8 \mathrm{~mm}$; $\mathrm{n}=1$ ); pes with well-developed interdigital membranes, reaching the middle of the first phalanges of dII to dIV; digits with sparse and moderately long ungual tufts (tufts appear to be short, since they not reach half the length of the claw, which is very long), not concealing claws; pes with very small interdigital pads; thenar and hypothenar pads present; plantar integument between pads densely covered by squamae; pads flesh colored. Tail much longer than head and body length; tail covered by short hairs, whose length equals the length of two scale rows on dorsal surface and of more than four scale rows on the ventral surface (forming a distinct keel on the ventral surface of tail, that act as a fin for navigation); apical tuft of hairs present, but quite small; scales very large and arranged in 10 transverse rows per centimeter at the base of the tail, in 11 rows per cm on the central portion of the tail, and in 12 rows on the apical centimeter of the tail ( $n=1)$; tail brownish (skin and hairs) dorsally, and light brownish ventrall, with whitish hairs in the keel.

Skull large and very robust (Fig. 33; Table 21). Rostrum short and wide; rostral tube absent. Nasals very long, expanded laterally distally, with posterior margin (nasofrontal suture) surpassing the maxillar-frontal-lacrimal sutures. Nasofrontal suture triangular and acute. Premaxillae not projected posteriorly, ending anteriorly to the nasals. Lacrimal predominantly in contact with the maxillar. Zygomatic notch very deep and wide. Interorbital region converging anteriorly, with supraorbital margins square or beaded, forming well developed dorsal projections, continuous with the sharp temporal margin. Frontosquamosal suture not collinear with the frontoparietal suture. Zygomatic arches robust, projected laterally, wider at the squamosal root. Braincase large and robust, with elongated profile. Parietals quite expanded ventrally, widely penetrating on the lateral surface of braincase. Rostrum with gnathic process short. Zygomatic plate very wide, with anterior margin straight or slightly concave; free dorsal margin projected anteriorly, with rounded anterodorsal margin sloped downwards. Zygomatic arches wide, with jugal large, and with maxillar and squamosal processes not overlapping. Stapedial and sphenofrontal foramina, and squamosal-alisphenoid groove absent; large opening for the alisphenoid canal; presence of the anastomotic canal on the surface of alisphenoid (derived carotid and stapedial circulatory pattern 3; Voss, 1988). Alisphenoid strut absent, configuring confluent oval accessory and buccinator-masticatory foramina. Postglenoid foramen present. Subsquamosal fenestra very small or absent. Stapedial and dorsostapedial processes of ectotympanic present; external auditory meatus small and rounded; mastoid completely ossified. Incisive foramina moderately long and narrow, with lateral margins parallel or slightly wider medially; anterior and posterior margins rounded, not reaching the anterior alveoli of M1. Posterior margin of zygomatic plate positioned anteriorly to the alveoli of M1. Palate wide and long, with central region deeper and with two deeper parallel depressions aligned with posterior palatine foramina (configuring a small medial crest on the posterior portion of the palate); palate with posterolateral palatal pits multiple, large and complex, recessed in deep palatal depressions. Mesopterygoid fossa not penetrating between molar series; roof of mestopterygoid fossa completely ossified, without sphenopalatine vacuities. Parapterygoid fossa narrower than mesopterygoid fossa. Auditory bullae small and moderately globose, with the periotic margin extending or not to the internal carotid canal. Mandible very robust, with high ramus (Fig. 33). Coronoid process wide, weakly falciform, lower than the condyloid process; angular process rounded, slightly surpassing posteriorly the condyloid process; superior notch and inferior notch very shallow. Capsular process of lower 


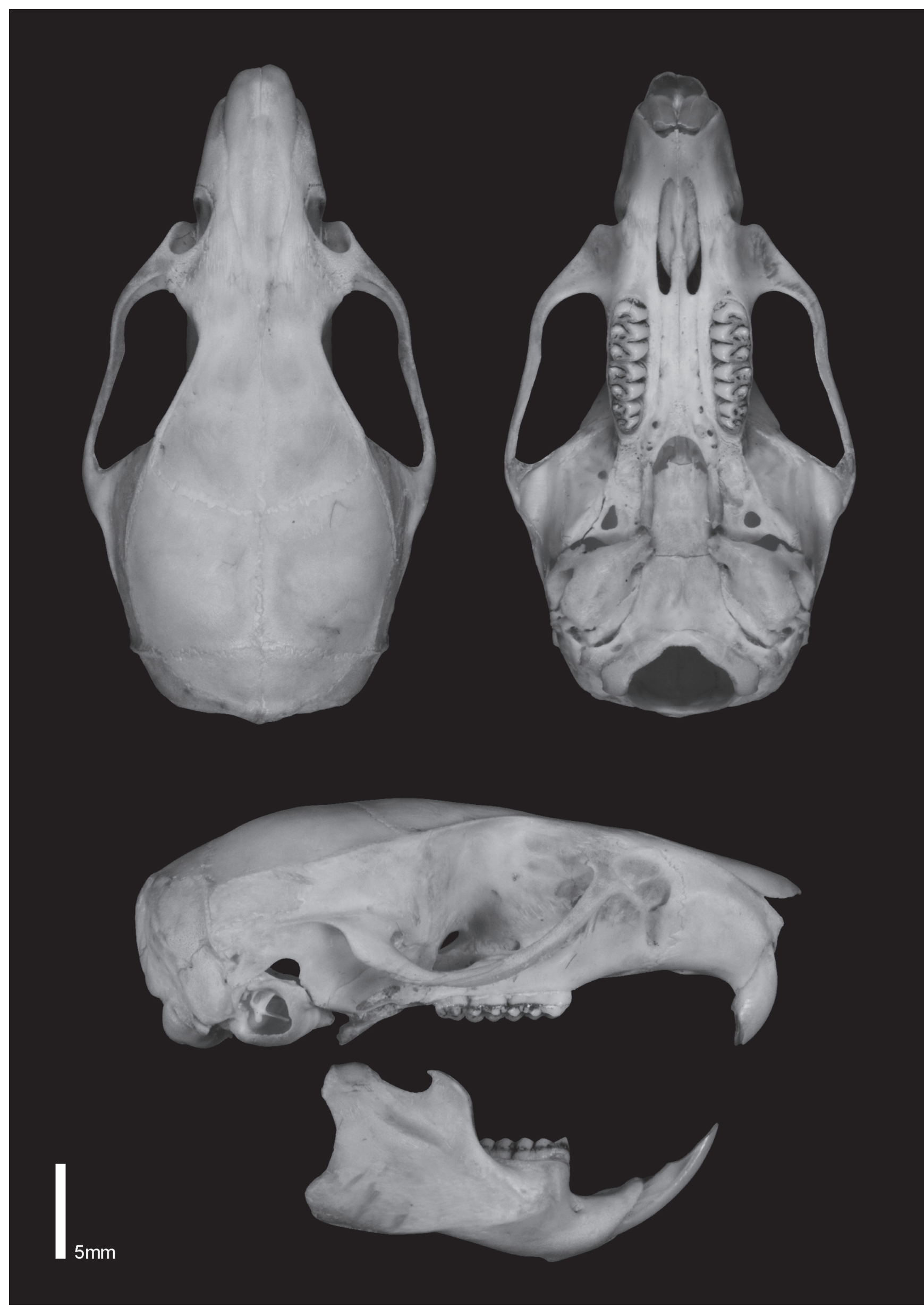

Figure 33. Skull and mandible of Nectomys squamipes (EEB 800; female; $G S L=35.29 \mathrm{~mm}$ ). Skull on dorsal, ventral, and lateral view, and mandible on lateral view. 
Table 21. Descriptive statistics (mean \pm standard deviation, range, and sample size) of cranio-dental measurements (mm) of Nectomys squamipes, obtained in Bonvicino (1994), and values of EEB specimens.

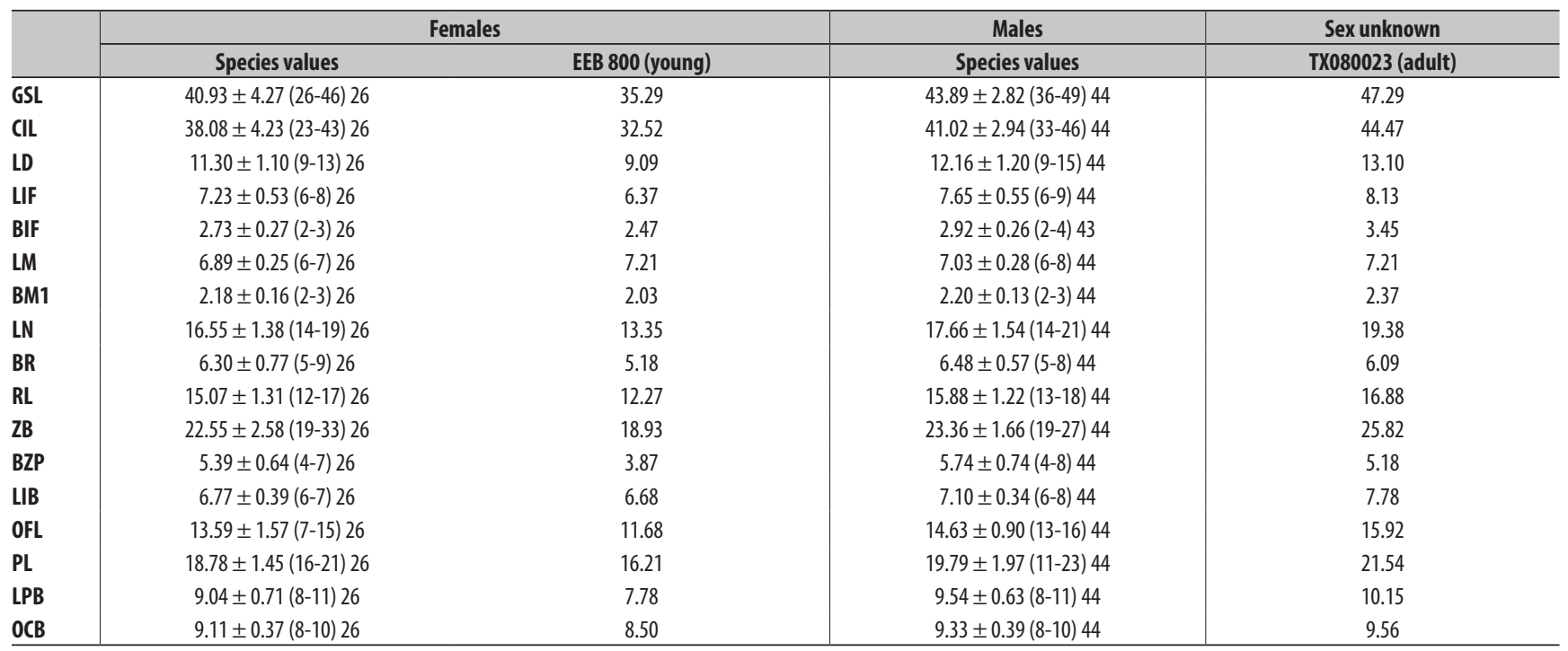

incisor absent or present, but small. Superior and inferior masseteric crests developed, forming a single crest anteriorly. Upper incisors opisthodont. Upper molar series parallel; molars with main cusps arranged in opposite pairs; molar series very robust, long and wide; labial and lingual flexus long (especially the labial) and deeply interpenetrating on medial plane of molar. M1 with procingulum not divided in two conules; anteroloph well developed, fused to the labial portion of the antecone, defining a long anterofosset; mesoloph present, reaching the labial cingulum, fused to the mesostyle; with minimum wear this flexus is closed labially by the fusion of the mesolophostyle to the paracone, forming a distinct mesofosset, that is bisected in two by an oblique paralophule near the labial margin of the fosset, originating two enamel islands (mesofossets: a smaller labial and a larger medial). $\mathrm{m} 1$ with procingulum not divided by the anteromedian flexid; anterolophid well developed, reaching the cingulid, and fused to the lingual portion of anterocone, defining a small anterofossetid; mesolophid well developed, fused to lingually to the entoconid, forming a entofossetid; protostylid and ectostylid small; ectolophid present.

Remarks: The morphological characteristics of the EEB specimens agree with the short description of $N$. squamipes provided by Bonvicino (1994), as well as the brief description of Hershkovitz (1944). The values of five (LM, LN, ZB, BZP, LPB) out of the 17 cranio-dental variables differed between EEB specimens and those studied by Bonvicino (1994) (see Table 21) - this may represent an intraspecific variation, or just a measurement discrepancy, since the values obtained from EEB specimens, although distinct, are very close to the values given by Bonvicino (1994).

Field observations: One of the specimens collected at EEB was a juvenile female with unperforated vagina, which was captured with pitfall trap in December 2003. For the other, no field information was recorded.

\section{Genus Oligoryzomys}

The genus Oligoryzomys is the most speciose and the most widely distributed member of the tribe Oryzomyini. It includes 19 species that occur from northern Central America to southern South America (Agrellos et al., 2012; Teta et al., 2013; Weksler \& Bonvicino, 2015). There is large intraspecific morphological variation within the genus as well as interspecific overlapping of morphological features, which makes challenging to delimit and identify species, frequently occurring in sympatry (Weksler \& Bonvicino, 2005; Paresque, 2010; Machado et al., 2011).

In the EEB samples, 108 specimens of Oligoryzomys were recovered, and to correctly identify these specimens at species level and to better understand the limits of morphologic variation within EEB populations, two discriminatory strategies were adopted: the first was a molecular approach using CYTB sequences of 14 specimens (including specimens that represented much of the morphological qualitative variation; data not shown); and the second was a morphometric approach using cranio-dental measurements from 65 specimens, including males and females. To optimize the morphometric analyses, we included eight specimens from Floresta Nacional de Ipanema, São Paulo, identified by Ana Paula Carmignotto using cytogenetic analyses - five as $O$. nigripes, with $2 n=62$, and three as $O$. flavescens, with $2 n=64-66$. We chose these two species as they are the most likely to occur in the area, based on the current taxonomic arrangements (Agrellos et al., 2012; Teta et al., 2013; Weksler \& Bonvicino, 2015).

The first morphometric analysis was a PCA including individuals from all age classes and 18 cranio-dental variables (Table 22). The results showed a weak separation among the species (Fig. 34), with specimens of $O$. flavescens appearing peripherally to, but in part superimposed with, specimens of $O$. nigripes, and predominantly with negative values along the first component. The first component was responsible for $77.89 \%$ of the 
Table 22. Scores of cranio-dental morphometric variables in the first two principal components from a PCA of Oligoryzomys. Variables that loaded higher on each component are indicated in bold.

\begin{tabular}{lcc}
\hline Variables & PC1 & PC 2 \\
\hline CIL & $\mathbf{0 . 9 9 3}$ & 0.019 \\
LD & $\mathbf{0 . 9 5 3}$ & -0.014 \\
LIF & 0.812 & -0.042 \\
BIF & 0.549 & 0.206 \\
LM & 0.330 & 0.026 \\
BM1 & 0.411 & 0.077 \\
LN & $\mathbf{0 . 9 0 4}$ & -0.270 \\
BR & 0.297 & $\mathbf{0 . 3 0 1}$ \\
RL & $\mathbf{0 . 9 5 8}$ & -0.067 \\
BZP & 0.761 & 0.095 \\
LIB & 0.277 & $\mathbf{0 . 4 8 3}$ \\
OFL & $\mathbf{0 . 9 1 1}$ & -0.029 \\
LPB & 0.681 & 0.048 \\
BBP & 0.711 & 0.142 \\
HBC & 0.347 & $\mathbf{0 . 5 1 3}$ \\
BBC & 0.600 & $\mathbf{0 . 5 9 1}$ \\
IPB & 0.274 & $\mathbf{0 . 8 2 5}$ \\
IPL & 0.234 & 0.243 \\
\hline
\end{tabular}

variation, and the variables that influenced this component more strongly were: condylo-incisive length (CIL), rostral length $(R L)$, length of diastema (LD), orbital fossa length (OFL) and length of nasals (LN). The second component corresponded to $6.76 \%$ of the variation and the determining variables were: interparietal breadth (IPB), breadth of braincase (BBC), height of braincase ( $\mathrm{HBC}$ ), least interorbital breadth (LIB) and breadth of rostrum (BR). The first two components were responsible for $84.65 \%$ of the variation. The variables that mostly influenced in each component suggest that the length of the cranium, more specifically the length of the rostrum, separates these two species; the second component indicates that this discrimination is based on the width of the braincase, and that the variables interparietal breadth

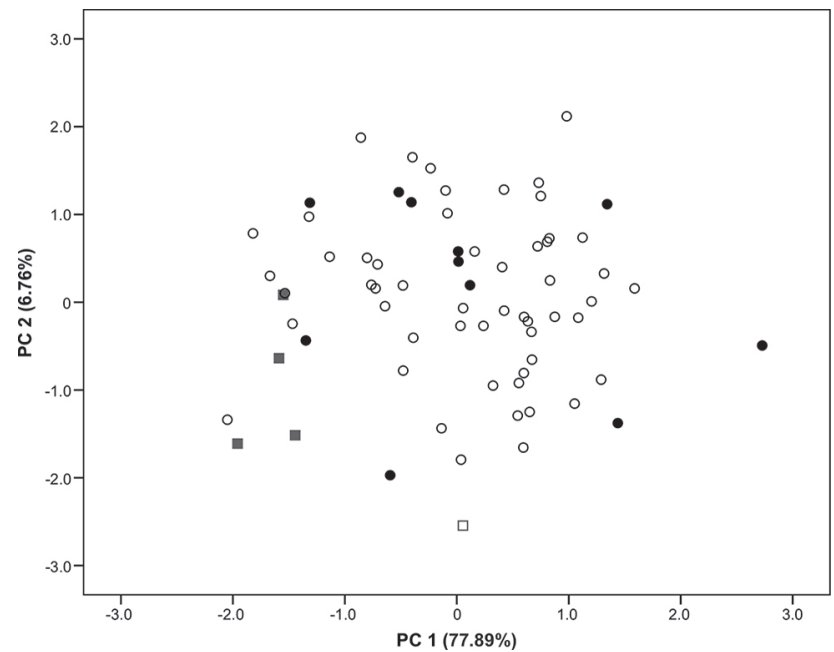

Figure 34. Scatterplot graph showing the projections of individual scores of the specimens of Oligoryzomys nigripes (dots; full black dots represent specimens with molecular or cytogenetic information) and Oligoryzomys flavescens (square; full gray dots represent specimens with molecular or cytogenetic information), from a PCA analysis. and breadth of braincase had more weight on the second component. The partial overlap between the two species can be explained by the fact that the O. flavescens individuals (in the lower portion of the graph) belong to AC2; they are superimposed with younger individuals of $O$. nigripes, usually in $A C 1$, demonstrating what was expected for the two species (Weksler \& Bonvicino, 2005; Machado et al., 2011): young individuals of $O$. nigripes are superimposed with older specimens of $O$. flavescens. It is important to note that the O. flavescens specimen that is separated from the other representative of the species (on the left of the graph) is an older individual. Therefore, the discrimination between individuals of $O$. flavescens corresponds to ontogenetic variation.

Additionally, we constructed bivariate graphs based on the values of the variables that strongly influenced the PCA results and variables that were potentially diagnostic for the species: CIL, RL, CZL, LN, BBP, BBC, BIF, BZP. The graphs of the Figs. 35A (CIL versus $\mathrm{RL}$ ) and 35B (CZL versus LN) were similar, showing a clear separation between most $O$. flavescens specimens from most specimens of $O$. nigripes, and one $O$. flavescens superimposed with
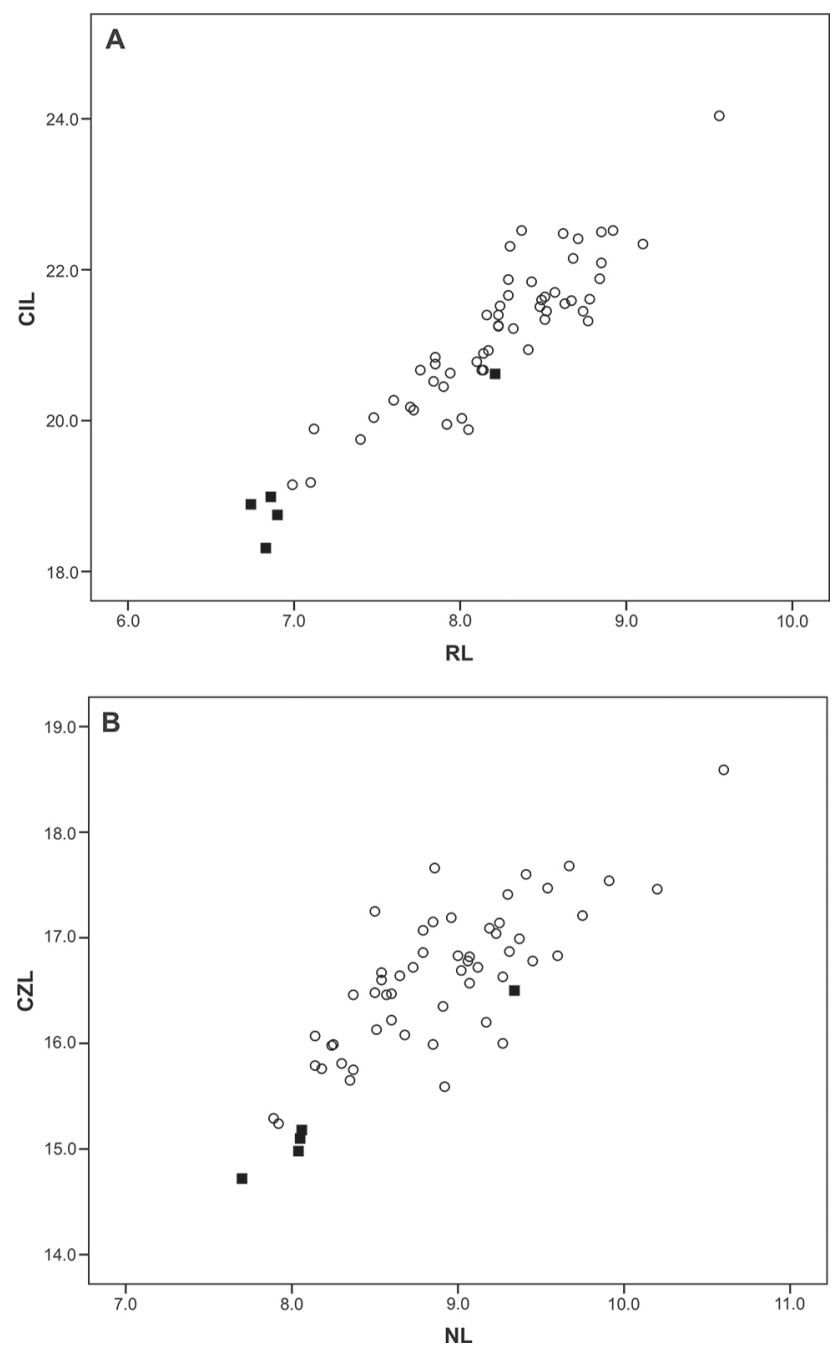

Figure 35. Bivariate graphs displaying (A) the condylo-incisive length (CIL) versus the rostral length (RL), and (B) the condylo-zigomatic length (CZL) versus the length of nasals (LN), of Oligoryzomys specimens. 0 . nigripes are represented by dots and 0 . flavescens by squares. 
O. nigripes. This specimen, however, corresponds the oldest individual of $O$. flavescens in the sample, and as in the case of the PCA, this result that may represent the superimposition of individuals of the two species that belong to different age classes. The other two graphs, BBP versus BBC (Fig. 36A) and BIF versus BZP (Fig. 36B), also showed similar results: the separation between $O$. flavescens and $O$. nigripes was greater and there was almost no superimposition between the specimens, which makes these variables important in the discrimination of the two species. The variables BIF and CIL had been recognized as being important to separate between the two species by Machado et al. (2011), based on individuals from Rio Grande do Sul.

Regarding qualitative morphological traits, some characters of external morphology were fundamental on the identification of these two species in the EEB samples, and, in general, they are consistent with previous suggestions of Weksler \& Bonvicino (2005), Paresque (2010) and Machado et al. (2011). For instance, the ventral coloration is entirely orange-cream in 0 . flavescens and predominantly whitish gray in $O$. nigripes, which also
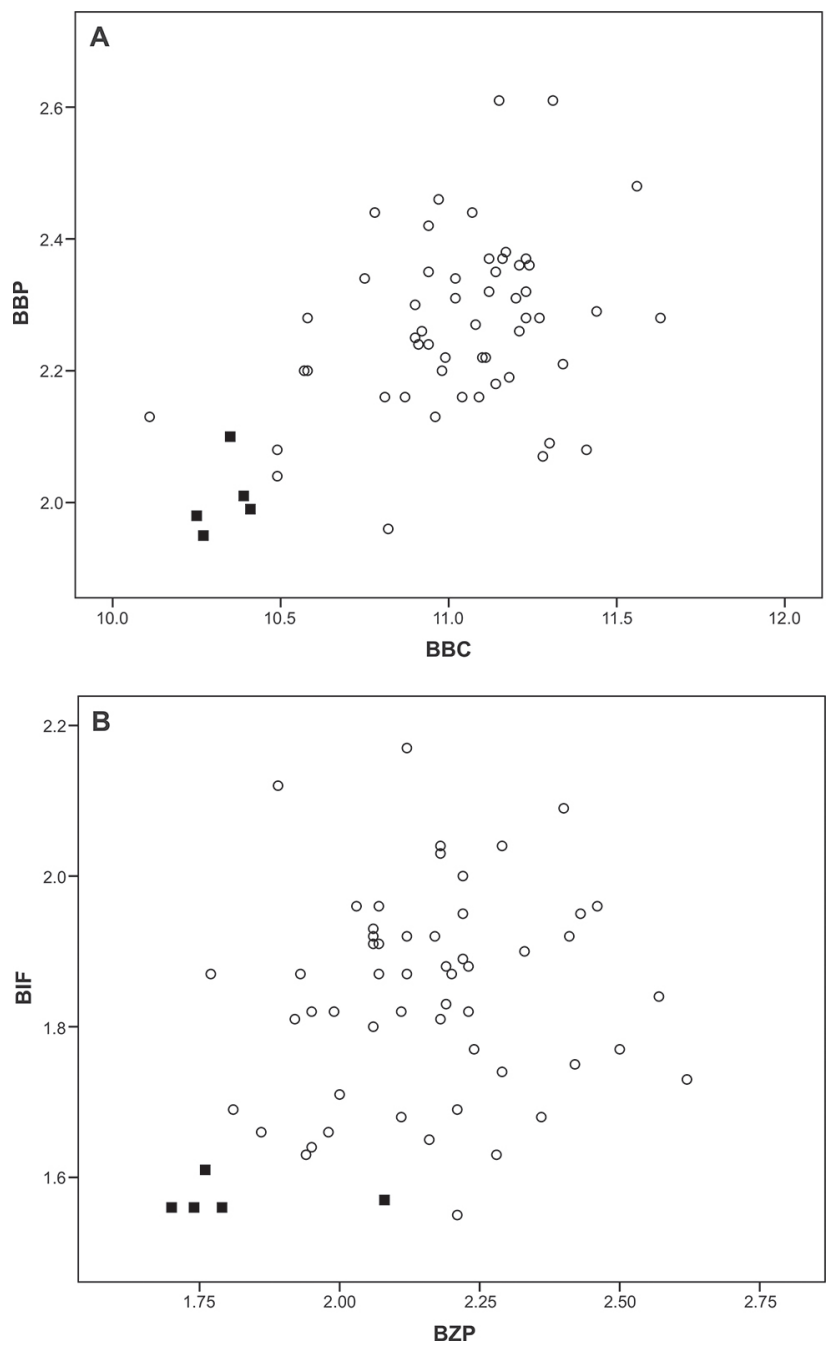

Figure 36. Bivariate graphs displaying $(A)$ the breadth of bony palate (BBP) versus the breadth of braincase $(\mathrm{BBC})$, and $(\mathrm{B})$ the breadth of incisive foramina (BIF) versus breadth of zygomatic plate (BZP), of Oligoryzomys specimens. 0 . nigripes are represented by dots and 0 . flavescens by squares. has a stripe of orange hairs in the pectoral region. Also, the most important character in the differentiation of the two specimens from EEB, was the transition in the dorsoventral coloration: the lateral coloration of $O$. nigripes is intensely orange on its limit with the venter, accentuating the dorsoventral transition; in O. flavescens, the lateral is weakly orange, whereas the venter is completely orange, making up for a subtle transition between the side and the venter. In specimens from EEB this characteristic can be unambiguously employed to separate between the two species.

In the skull, we also corroborate some diagnostic features for these species as already proposed (e.g., Bonvicino \& Weksler, 1998; Weksler \& Bonvicino, 2005; Paresque, 2010; Machado et al., 2011), such as: shape of incisive foramen, shape of interorbital region, orientation of foramen magnum, shape of zygomatic arch and braincase (see specimen identifications below). However, some discrepancies between the characters observed in the EEB specimens and the descriptions in the literature were found, especially in the cranial morphology, such as: zygomatic arch more delicate and less divergent in $O$. flavescens versus more robust and more divergent in $O$. nigripes; braincase more rounded in $O$. flavescens and less rounded in O. nigripes; foramen magnum more ventrally oriented in $O$. flavescens adults and more posterior oriented in adults of $O$. nigripes (as described by Machado et al., 2011), interorbital region narrower medially in O. flavescens and narrower along the molar series in O. $n$ igripes (as reported by Paresque, 2010). It is possible that the small sample of $O$. flavescens - only two with cranium available for analysis - have contributed to our inability to discern these overall patterns. The O. nigripes sample, by contrast, was larger and it allowed us to observe great intraspecific cranial plasticity (presented below), which includes even characters that had been previously recognized as diagnostic for O. flavescens. Therefore, of the 108 specimens, we assigned three to $O$. flavescens and 105 to O. nigripes.

\section{Oligoryzomys flavescens (Waterhouse 1837)}

Voucher material: $\mathrm{n}=3$; male: uncatalogued: EBM 335; EEB 1008; sex unknown: uncatalogued: EEB 675.

Specimens identification: Small size (see body measurements on Table 23). Dorsal pelage dense and soft, moderately lax; dorsal pelage orange or ocher grizzled with black hairs, with hump more orangish. Dorsal pelage with guard hairs moderately long and stiff (length ranging from 11 to $13 \mathrm{~mm}$ ), with proximal half thinner and grayish and distal portion wider and black; cover hairs shorter (length ranging from 9 to $9.5 \mathrm{~mm}$ ), with proximal portion ( $3 / 5$ to $4 / 5)$ grayish and distal portion banded, with basal band orange and terminal band black; wool hairs very short, wavy and thin (length ranging from 6.5 to $7.5 \mathrm{~mm}$ ), gray basally and with the apical portion banded, with basal band orange and terminal band black. Lateral pelage predominantly orange more or less intensely griz- 
Table 23. Descriptive statistics (mean \pm standard deviation, range, and sample size) of body measurements $(\mathrm{mm})$, and weight $(\mathrm{g}$ ), of adult specimens of Oligoryzomys flavescens and 0. nigripes from EEB, and comparative descriptive statistics obtained in *Weksler \& Bonvicino (2005) and ** Machado et al. (2011).

\begin{tabular}{|c|c|c|c|c|c|c|}
\hline & \multicolumn{3}{|c|}{ Oligoryzomys flavescens } & \multicolumn{3}{|c|}{0. nigripes } \\
\hline & Species values* & Values of specimens from $\mathrm{RS} * *$ & EEB specimens & Species values* & Values of specimens from $\mathrm{RS}^{* *}$ & EEB specimens \\
\hline HBL & $87.8 \pm 5.7(81.0-93.0) 4$ & $70.75(60.0-86.0) 12$ & $(85.0-94.0) 2$ & $92.0 \pm 9.9(70.0-120.0) 44$ & $85.08(62.0-115.0) 88$ & $86.46 \pm 4.57(81.0-96.0) 23$ \\
\hline TL & $109.7 \pm 17.7(97.0-130.0) 4$ & $104.08(81.0-115.0) 12$ & $(99.6-104.0) 2$ & $116.0 \pm 10.5(96.0-144.0) 43$ & $121.57(88.0-140.0) 82$ & $119.73 \pm 6.89(103.9-131.5) 23$ \\
\hline HF1 & - & $22.07(20.0-28.0) 14$ & $(22.0-22.3) 2$ & - & $23.77(21.0-28.0) 86$ & $22.94 \pm 1.11(20.8-24.8) 22$ \\
\hline HF2 & $23.5 \pm 13(22.0-24.0) 4$ & $23.57(21.0-30.0) 14$ & $(23.0) 2$ & $24.8 \pm 1.8(21.0-29.0) 42$ & $25.38(22.0-30.0) 85$ & $24.12 \pm 1.04(21.9-26.0) 23$ \\
\hline EL & $15.0 \pm 0.8(15.0-16.0) 4$ & $13.14(12.0-15.0) 14$ & $(11.0-13.0) 2$ & $17.0 \pm 1.4(14.5-20.0) 44$ & $15.83(10.0-20.0) 87$ & $14.83 \pm 1.49(12.0-19.0) 23$ \\
\hline W & $18.8 \pm 2.6(15.0-21.0) 4$ & - & $(10.0-17.5) 2$ & $25.4 \pm 4.7(18.0-40.0) 29$ & - & $19.04 \pm 3.73(14.0-27.0) 13$ \\
\hline
\end{tabular}

zled with brown, more intensely orangish on cheeks and rump; transition with ventral region subtle. Ventral pelage predominantly orangish cream, with gray-based (1/2) orange cream hairs. Mystacial vibrissae moderately long and dense, reaching the base of pinnae when laid backwards. Pinnae small and rounded, covered internally by short hairs with brown base and orange tip, and covered externally by short hairs, entirely black or orange. Manus covered dorsally by predominant short white or yellowish hairs and few grayish brown hairs; dII to dV without conspicuous ungual tufts; digits with short claws (claw of dIII with 1.1 to $1.5 \mathrm{~mm} ; \mathrm{n}=2$ ). Pes long and narrow, with dorsal surface covered by short white or yellowish hairs; dIII similar to digit IV and longer than digit II; claw moderate in size (claw of dIII with 2.2 to $2.5 \mathrm{~mm} ; \mathrm{n}=2$ ); digits with short and sparse white ungual tufts; pes with small interdigital pads; thenar and hypothenar pads present; plantar integument between pads with squamae; pads with the same color as the remaining ventral surface. Tail longer than head and body $(127.8 \% \pm 24.3$ of head and body length, ranging from 110.6 to $155.6 \% ; n=3$ ); tail covered by short hairs, whose length equals the length of one and a half to two scale rows on dorsal surface and of more than two scale rows on the ventral surface; apical tuft of hairs absent, but longer hairs are present on the tip of the tail; scales very small and arranged in 17 transverse rows per centimeter at the base of the tail, between 16 to 17 rows per $\mathrm{cm}$ on the central portion of the tail, and in 28 rows on the apical centimeter of the tail $(n=2)$; tail brownish (skin and hairs) dorsally, and ventrally whitish; tail distinctly bicolored.

Skull short, small and delicate (Fig. 37; Table 24). Rostrum moderately short and narrow; rostral tube absent. Nasals long, expanded laterally distally, with anterior margin projected beyond the superior incisors, and with posterior margin (nasofrontal suture) surpassing the maxillar-frontal-lacrimal sutures. Nasofrontal suture rounded. Premaxillae projected posteriorly, aligned to

Table 24. Descriptive statistics (mean \pm standard deviation, range, and sample size) of cranio-dental measurements (mm) of adult specimens of Oligoryzomys flavescens and 0. nigripes from EEB, and comparative descriptive statistics obtained in *Weksler \& Bonvicino (2005) and **Machado et al. (2011).

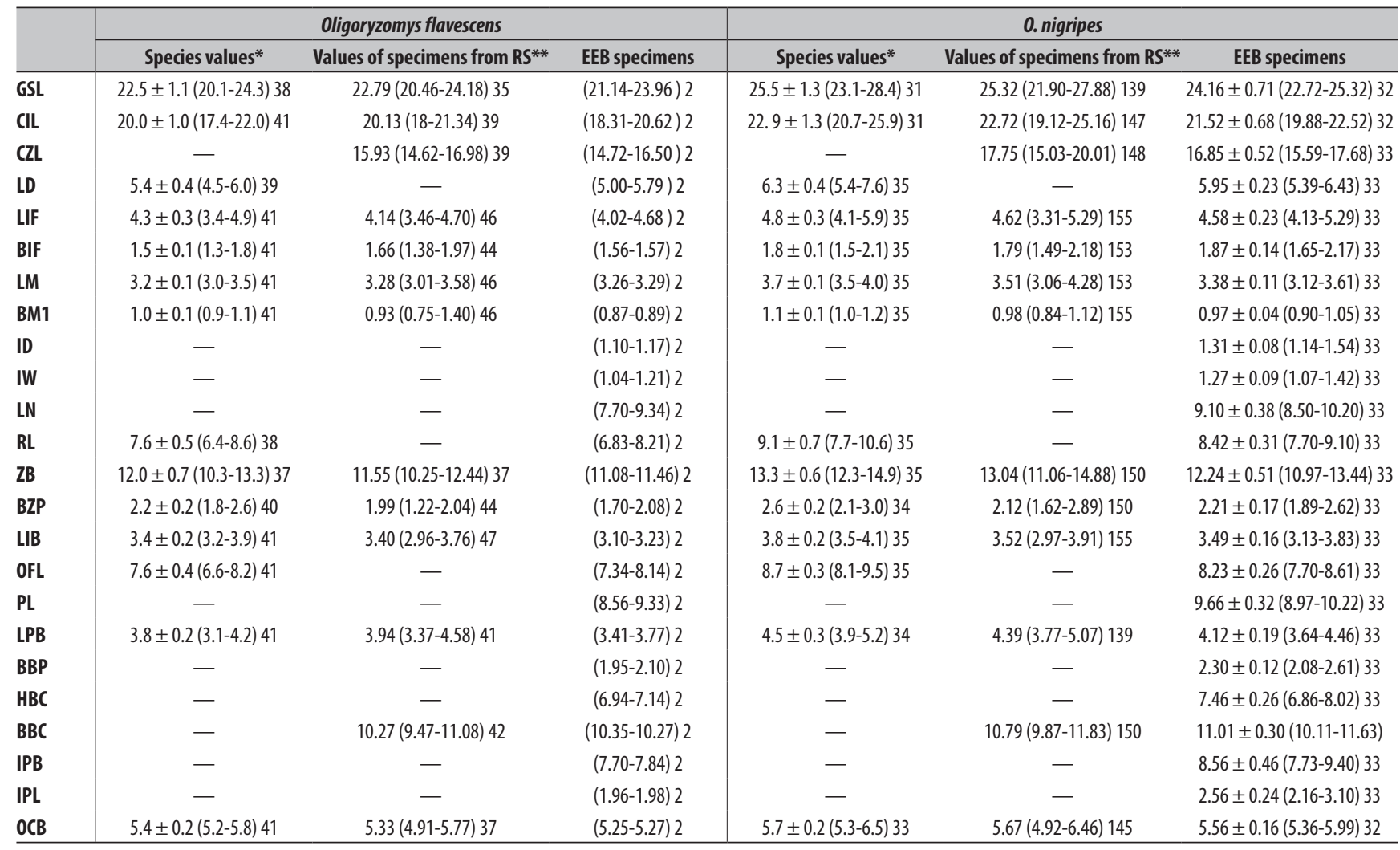




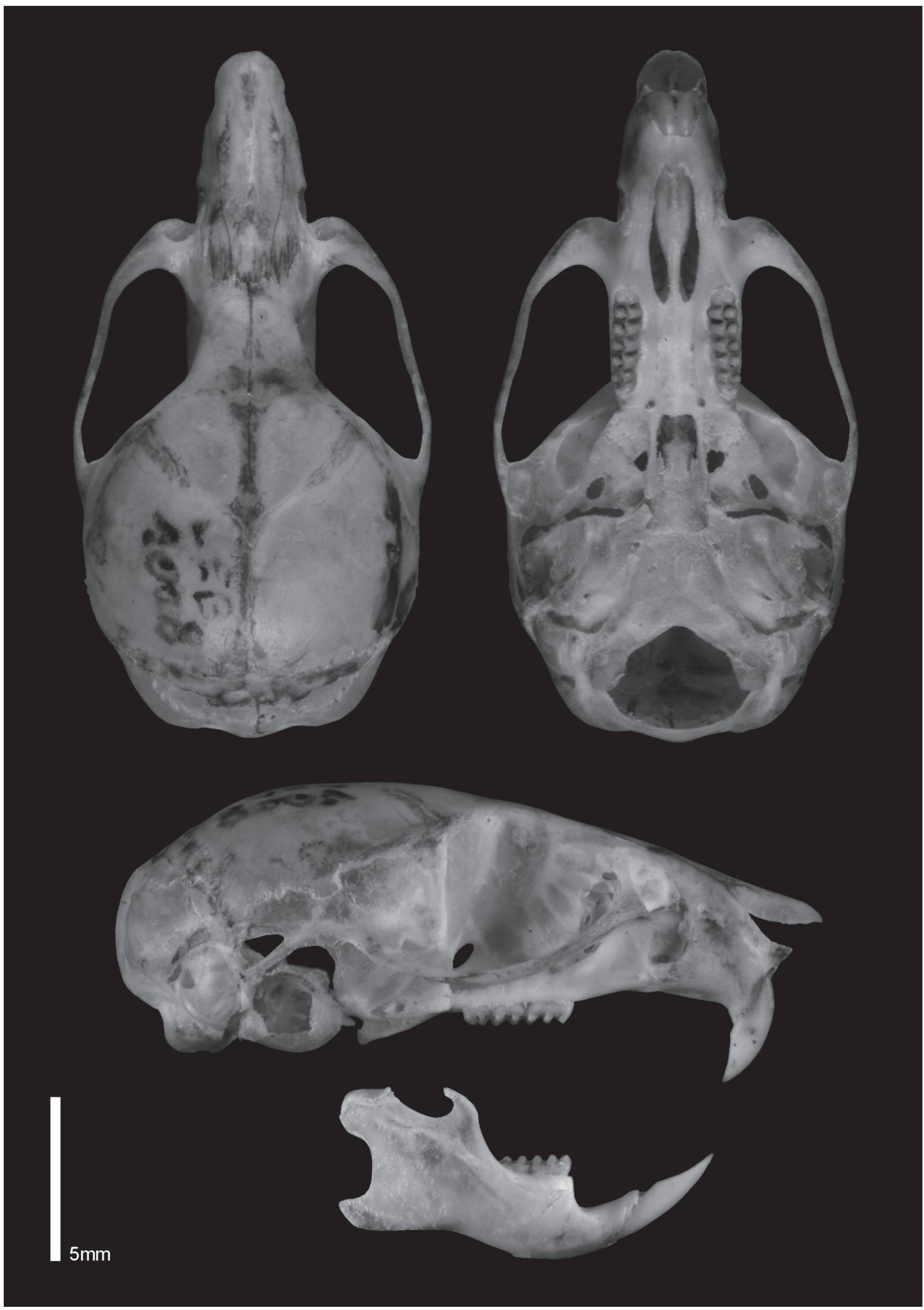

Figure 37. Skull and mandible of Oligoryzomys flavescens (EEB 1008; male; GSL=21.14 mm). Skull on dorsal, ventral, and lateral view, and mandible on lateral view. 
the nasals or surpassing them. Lacrimal equally contacts maxillar and frontal. Zygomatic notch shallow and moderately narrow. Interorbital region symmetrically constricted, narrower medially, with supraorbital margins squared, continuous with the moderately sharp temporal margin. Frontosquamosal suture collinear with the frontoparietal suture. Zygomatic arches projected laterally, parallel, slightly wider at the squamosal root. Braincase large, with rounded (young specimen) or elongated (adult) profile. Parietals slightly expanded ventrally, on the lateral surface of braincase. Interparietal long and wide. Rostrum with gnathic process short; rostral tube absent. Zygomatic plate moderately narrow, with anterior margin straight; free dorsal margin not distinctly projected anteriorly, with squared anterodorsal margin. Zygomatic arches narrow, with jugal absent and with maxillar and squamosal processes in contact. Stapedial foramen large; sphenofrontal foramen, squamosal-alisphenoid groove, and anastomotic canal absent (derived carotid and stapedial circulatory pattern 2; Voss, 1988). Alisphenoid strut absent, configuring confluent oval accessory and buccinator-masticatory foramina (a single foramen ovale). Postglenoid foramen present. Subsquamosal fenestra present. Stapedial process of ectotympanic present; external auditory meatus small and rounded. Mastoid with fenestra. Incisive foramina long and narrow, with lateral margins parallel or slightly wider medially, and anterior and posterior margins rounded; posterior margin reaching the anterior alveoli of $\mathrm{M} 1$ or penetrating between the molar series, reaching the anterolingual conule. Posterior margin of zygomatic plate positioned anteriorly to the alveoli of M1. Palate wide and long, with medial region deeper and with two deeper parallel depressions aligned with posterior palatine foramina (creating a small medial crest); palate with posterolateral palatal pits deep and complex situated in moderately deep palatal fossa. Mesopterygoid fossa not penetrating between molar series; roof of mestopterygoid fossa perforated by large sphenopalatine vacuities, along the presphenoid and basisphenoid. Parapterygoid fossa wider than the mesopterygoid fossa. Auditory bullae small and moderately globose, with the periotic margin extending to the internal carotid canal. Mandible delicate, with ramus low (Fig. 37). Coronoid process high and weakly to moderately falciform, equal in height to the condyloid process; angular process rounded, on the same level of the condyloid process; superior notch and inferior notch shallow. Capsular process of lower incisor present and well developed, large. Superior and inferior masseteric crests weakly developed (inferior more developed) and converging anteriorly in a V-shape. Upper incisors opisthodont. Upper molar series parallel or slightly convergent anteriorly; molars with main cusps arranged in opposite pairs; labial and lingual flexus slightly interpenetrating on medial plane of molar. M1 with procingulum divided by the anteromedian flexus in anterolingual and anterolabial conules (anterolabial conule larger than the anterolingual); anteroloph well developed, reaching the labial margin and labial cingulum; anteroflexus narrow and short; paracone fused medially to the protocone, forming a long paraflexus; mesoloph present, reaching the labial cingulum, fused to the mesostyle; with wear this flexus will be closed, forming a distinct mesofosset; protostyle and enterostyle present. $\mathrm{m} 1$ with procingulum divided by the anteromedian flexid, in anterolingual and anterolabial conulids; anterolophid well developed, reaching the cingulid; mesolophid well developed, fused to mesostylid; protostylid well developed; ectostylid present (young specimen) and ectolophid present (adult specimen).

Field observations: Three specimens of $O$. flavescens were collected with pitfall traps at EEB throughout the sampling period: one adult (sex unknown) in December, 2003; a male with scrotal testes in April, 2004; and juvenile male in December, 2011.

Additional specimens examined: Oligoryzomys flavescens: $\mathrm{n}=3$; Brazil: São Paulo: Iperó: Floresta Nacional de Ipanema $\left(23^{\circ} 25^{\prime} 49^{\prime \prime} \mathrm{S}, 47^{\circ} 37^{\prime} 22^{\prime \prime} \mathrm{W}\right)$ : male: uncatalogued: APC 1705, 1779, 1860.

\section{Oligoryzomys nigripes (Olfers 1818)}

Voucher material: $\mathrm{n}=105$; female: MZUSP 33730, 33731; uncatalogued: EBM 014, 020, 025, 047, 048, 051, $075,100,114,115,126,141,162,166,181,182,188,206$, 227, 236, 248, 256, 259, 260; EEB 518, 528, 746, 754, 814, $817,827,828,829,842,916$; male: MZUSP 33732, 33733, 33734, 33735, 33744, 33745, 33746; uncatalogued: EBM 003, 008, 022, 024, 042, 050, 055, 060, 069, 094, 096, 098, $101,108,109,117,125,139,146,147,152,183,209,210$, $211,214,223,224,226,228,235,237,238,240,241,245$, 254, 257, 306; EEB 546, 673, 714, 763, 774, 811, 815, 820, $830,835,839,840,845,846,847,861,867,1019$; sex unknown: uncatalogued: EBM 062; EEB 719, 882, 888.

Specimens identification: Small size (see body measurements on Table 23). Dorsal pelage short, moderately dense and harsher; dorsal pelage orange or ocher grizzled with brown hairs. Dorsal pelage with guard hairs moderately long and stiff (length ranging from 9.5 to $12 \mathrm{~mm}$ ), with half proximal portion thinner and grayish and distal portion wider and black; cover hairs shorter (length ranging from 7 to $8.5 \mathrm{~mm}$ ), with proximal portion $(2 / 3)$ grayish and distal portion banded, with basal band orange and terminal band black; wool hairs very short, wavy and thin (length ranging from 5.5 to $6.5 \mathrm{~mm}$ ), gray basally (3/4) and with the apical portion banded, with basal band orange and terminal band black. Lateral pelage predominantly yellow or orange more or less intensely grizzled with brown, more intensely orange on the transition line with the ventral region; transition with ventral region sharp. Ventral pelage predominantly grayish, grayish-white to grayish yellow; several specimens with a transverse orange band along the pectoral region; hairs with gray base $(2 / 3)$ and white, cream white or yellowish white tip. Mystacial vibrissae moderately long and dense, reaching the base of pinnae when laid 
backwards. Pinnae small and rounded, densely covered internally by short hairs with brown base and orange tip, and weakly covered externally by short hairs, entirely brown. Manus short and narrow, covered dorsally by predominant short white hairs and brown base hairs; dll to dV without conspicuous ungual tufts; digits with short claws (claw of dIII with 1.1 to $1.5 \mathrm{~mm} ; \mathrm{n}=4$ ). Pes long and narrow, with dorsal surface covered by short white hairs and brown base hairs; dIII similar to dIV and longer than digit II; claw moderate in size (claw of dIII with 2.1 to $2.3 \mathrm{~mm} ; \mathrm{n}=4$ ); digits with moderately long and dense white ungual tufts, surpassing and concealing claws; pes with small interdigital pads; thenar and hypothenar pads present; plantar integument between pads with squamae; pads with the same color as the remaining ventral surface. Tail much longer than head and body $(139.7 \% \pm 11.8$ of head and body length, ranging from 113 to $172 \% ; n=80$ ); tail covered by short hairs, whose length equals the length of one to two scale rows on dorsal surface and of more than two scale rows on the ventral surface; apical tuft of hairs absent; scales very small and arranged in 15 to 18 transverse rows per centimeter at the base of the tail, between 14 to 21 rows per $\mathrm{cm}$ on the central portion of the tail, and between 19 to 25 rows on the apical centimeter of the tail $(n=4)$; tail brownish (skin and hairs) dorsally, and ventrally whitish (proximal half) and brownish (distal half); tail bicolored on proximal half.

Skull short, small and delicate (Fig. 38; Table 24). Rostrum moderately short and narrow; rostral tube absent. Nasals long, expanded laterally distally, with anterior margin projected beyond the superior incisors, and with posterior margin (nasofrontal suture) surpassing $(74.2 \%)$ or not $(25.8 \%)$ the maxillar-frontal-lacrimal sutures $(n=62)$. Nasofrontal suture rounded $(69.4 \%)$, acute $(11.3 \%)$ or squared $(19.4 \%)(n=62)$. Premaxillae projected posteriorly, aligned to the nasals (51.6\%) or surpassing the nasals (37.1\%), or not projected posteriorly, with premaxillar/frontal suture anterior to nasofrontal suture $(11.3 \%)(n=62)$. Lacrimal equally contacts maxillar and frontal $(96 \%)$ or mainly in contact with maxillar (4\%) $(n=62)$. Zygomatic notch shallow to moderately deep and narrow. Interorbital region symmetrically constricted, narrower medially, with supraorbital margins squared $(90.5 \%)$ or rounded $(9.5 \%)$, continuous with the moderately sharp temporal margin $(n=62)$. Frontosquamosal suture collinear $(68.3 \%)$ or not $(31.7 \%)$ with the frontoparietal suture $(n=62)$. Zygomatic arches projected laterally, parallel, slightly wider at the squamosal root. Braincase large, with rounded or elongated profile. Parietals restricted to the dorsal surface of the braincase (3.2\%), predominantly associated to the dorsal surface of braincase $(85.5 \%)$ or slightly expanded ventrally, on the lateral surface of braincase $(11.3 \%)(n=62)$. Interparietal long and wide. Rostrum with gnathic process short. Zygomatic plate moderately narrow, with anterior margin flat $(79.4 \%)$ or slightly wider, with anterior margin projected anteriorly (20.6\%) $(n=62)$. Zygomatic arches narrow, with jugal absent and with maxillar and squamosal processes in contact. Stapedial foramen large; sphe- nofrontal foramen, squamosal-alisphenoid groove, and anastomotic canal absent (derived carotid and stapedial circulatory pattern 2; Voss, 1988). Alisphenoid strut absent, configuring confluent oval accessory and buccinator-masticatory foramina (a single foramen ovale) (93.7\%) or present as a narrow strut (only on the left side of the skull; 6.3\%), configuring separated oval accessory and buccinator-masticatory foramina $(n=62)$. Postglenoid foramen present. Subsquamosal fenestra present. Stapedial process of ectotympanic present; external auditory meatus small and rounded. Mastoid with fenestra. Incisive foramina long and narrow, with lateral margins parallel (33.3\%) or slightly wider posteriorly $(66.7 \%)$, and anterior and posterior margins rounded $(n=63)$; posterior margin distant of the M1 (34.9\%), reaching the anterior alveoli of $\mathrm{M} 1$ (63.5\%), penetrating between the molar series $(1.6 \%)$, reaching the anterolingual conule $(n=63)$. Posterior margin of zygomatic plate positioned anteriorly to the alveoli of M1. Palate wide and long, with central region deeper and with two deeper parallel depressions aligned with posterior palatine foramina (configuring a small medial crest); palate with posterolateral palatal pits multiple, deep and complex situated in moderately deep palatal fossa $(65.1 \%)$, situated on the palate level (14.3\%), or as a single and large posterolateral palatal pit (20.6\%) ( $n=63$ ). Mesopterygoid fossa not penetrating between molar series; anterior margin of mesopterygoid fossa squared $(53.3 \%)$ or rounded $(46.7 \%)(n=63)$; roof of mestopterygoid fossa perforated by large sphenopalatine vacuities, along the presphenoid and basisphenoid. Parapterygoid fossa wider than the mesopterygoid fossa. Auditory bullae small and moderately globose, with the periotic margin extending to the internal carotid canal. Mandible delicate, with ramus low (Fig. 38). Coronoid process low and weakly to moderately falciform, lower than the condyloid process; angular process rounded, on the same level of the condyloid process; superior notch shallow and inferior notch moderately deep. Capsular process of lower incisor present and well developed, large. Superior and inferior masseteric crests weakly developed (inferior more developed) and converging anteriorly in a V-shape. Upper incisors opisthodont. Upper molar series parallel or slightly convergent anteriorly; molars with main cusps arranged in opposite pairs; labial and lingual flexus slightly interpenetrating on medial plane of molar. M1 with procingulum divided by the anteromedian flexus in anterolingual and anterolabial conules; anteroloph well developed, reaching the labial margin and labial cingulum; anteroflexus narrow and short; paracone fused medially to the protocone, forming a long paraflexus; mesoloph present, reaching the labial cingulum, fused to the mesostyle; with wear this flexus will be closed, forming a distinct mesofosset; protostyle and enterostyle present. $\mathrm{m} 1$ with procingulum divided by the anteromedian flexid, in anterolingual and anterolabial conulids; anterolophid well developed, reaching the cingulid; mesolophid well developed, fused to mesostylid; protostylid well developed; ectostylid present (young specimen) and ectolophid present (adult specimen). 


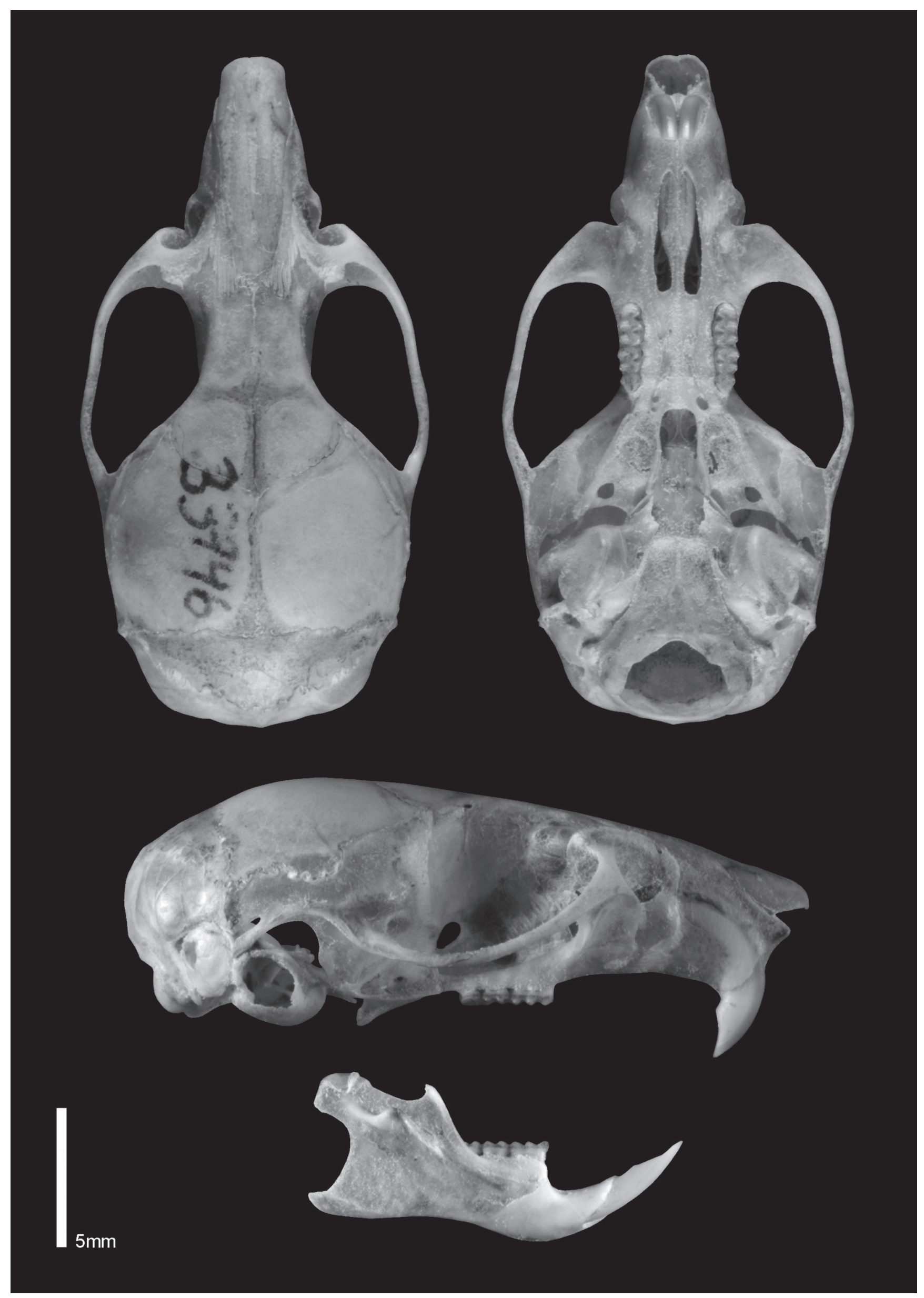

Figure 38. Skull and mandible of Oligoryzomys nigripes (MZUSP 33746; male; GSL=24.33 mm). Skull on dorsal, ventral, and lateral view, and mandible on lateral view. 
Ontogenetic and sexual variation: Using the cranio-dental morphometric variables we analyzed the pattern of cranial ontogenetic development and the presence of sexual dimorphism in different age classes of specimens of Oligoryzomys nigripes from EEB $(n=63)$. The specimens examined were sorted in four age classes: AC1 $(n=14), \operatorname{AC2}(n=18), A C 3(n=27)$ and AC4 $(n=4)$. The 25 cranio-dental morphometric variables were tested and presented a normal distribution according to the Kolmogorov-Smirnov test (Table 25). ANOVA followed by Tukey's post hoc tests were used to ascertain how these variables behave ontogenetically. A total of 18 out of 25 variables differed significantly between age classes and conformed to a similar growth pattern: 12 of the 18 variables increased, on average, with age; these variables were correlated with the length of the cranium (GSL, CIL, CZL, LD, OFL and LPB), upper incisors (ID and IW), width of the cranium or width of cranial structures (ZB, BBP, BZP and $O C B$ ); five of the 18 variables, from the rostral region of the cranium (LIF, BIF, LN, BR and PL), increased in value with age, except between AC3 and AC4, where their averages decreased. This needs to be interpreted with caution given the small numbers of specimens available in AC4; additionally, the mean of the variable BBC increased significantly from AC1 to AC2 and then in older age classes it did not vary significantly. The remaining seven variables that did not vary significantly among age classes are related to the upper molars (LM and BM1), width of cranium (BR and LIB), interparietal (IPB and IPL) and height of braincase (HBC) (Table 25).
The comparisons between the four age classes reveals that individuals of $A C 1$ differ from individuals of $A C 2$ in 15 variables; from AC3 individuals in 17 variables, and from AC4 specimens in 15 variables; individuals of AC2 differ from individuals of AC3 in nine variables and from AC4 individuals in three variables; and, finally, specimens of AC3 do not differ significantly from specimens of AC4 (Table 25) in any variable. In this manner, specimens of $O$. nigripes from EEB can be classified into three distinct age-groups: juveniles (AC1); subadults (AC2); and adults (AC3 and AC4).

These results are consistent with those found by Prado \& Percequillo (2011) for Aegialomys; Carleton \& Musser (1989) for Microryzomys; and Voss (1991) for Zygodontomys: all of them observed great variation in size, with mean values of most of the variables increasing from the younger to the next, older age classes, except for variables of the upper molars (LM and BM1) and neurocranium (represented here by $B R, L I B, I P B, I P L, H B C$ ). The absence of ontogenetic variation in the molars and neurocranium is due to the fast development of these structures just after birth in these animals. On the other hand, the craniofacial variables and those of the incisors have great ontogenetic variation due to the continuous growth of these structures with age, as found by us for many variables discussed above.

Sexual dimorphism was tested with the Student's t-test, by comparing males and females within each age class established by the ontogenetic variation analysis. The results show that there is no cranio-dental sexual di-

Table 25. Results of Kolmogorov-Smirnov test, Analysis of Variance (ANOVA), and Tukey's post-hoc test, and sample size in each age class for 25 cranio-dental morphometric variables measured in Oligoryzomys nigripes from EEB. Statistically significant values $(P<0.05)$ are indicated in bold.

\begin{tabular}{|c|c|c|c|c|c|c|c|c|c|c|c|c|c|}
\hline & \multirow{2}{*}{$\begin{array}{l}\text { K-S } \\
\text { Sig. }\end{array}$} & \multicolumn{2}{|c|}{ ANOVA } & \multicolumn{6}{|c|}{ Tukey } & \multicolumn{4}{|c|}{$\mathbf{N}$} \\
\hline & & $\mathbf{F}$ & Sig. & 1 vs. 2 & 1 vs. 3 & 1 vs. 4 & 2 vs. 3 & 2 vs. 4 & 3 vs. 4 & 1 & 2 & 3 & 4 \\
\hline GSL & 0.860 & 29.016 & 0.000 & 0.000 & 0.000 & 0.000 & 0.001 & 0.095 & 1.000 & 13 & 18 & 27 & 4 \\
\hline CIL & 0.280 & 31.621 & 0.000 & 0.000 & 0.000 & 0.000 & 0.003 & 0.110 & 0.990 & 13 & 18 & 27 & 4 \\
\hline CZL & 0.530 & 27.038 & 0.000 & 0.000 & 0.000 & 0.000 & 0.003 & 0.076 & 0.962 & 12 & 18 & 27 & 4 \\
\hline LD & 0.361 & 28.835 & 0.000 & 0.000 & 0.000 & 0.000 & 0.001 & 0.088 & 0.997 & 14 & 18 & 27 & 4 \\
\hline LIF & 0.518 & 17.702 & 0.000 & 0.000 & 0.000 & 0.004 & 0.035 & 0.796 & 0.919 & 14 & 18 & 27 & 4 \\
\hline BIF & 0.644 & 6.040 & 0.001 & 0.083 & 0.001 & 0.130 & 0.334 & 0.893 & 0.996 & 14 & 18 & 27 & 4 \\
\hline LM & 0.410 & 1.668 & 0.184 & - & - & - & - & - & - & 12 & 18 & 27 & 4 \\
\hline BM1 & 0.330 & 2.101 & 0.110 & - & - & - & - & - & - & 14 & 18 & 27 & 4 \\
\hline ID & 0.752 & 18.983 & 0.000 & 0.001 & 0.000 & 0.000 & 0.020 & 0.175 & 0.972 & 14 & 18 & 27 & 4 \\
\hline IW & 0.676 & 16.383 & 0.000 & 0.438 & 0.000 & 0.003 & 0.000 & 0.038 & 1.000 & 14 & 18 & 27 & 4 \\
\hline LN & 0.967 & 29.799 & 0.000 & 0.000 & 0.000 & 0.000 & 0.000 & 0.482 & 0.671 & 13 & 18 & 27 & 4 \\
\hline BR & 0.391 & 0.450 & 0.718 & - & - & - & - & - & - & 13 & 18 & 27 & 4 \\
\hline RL & 0.527 & 26.158 & 0.000 & 0.000 & 0.000 & 0.000 & 0.001 & 0.164 & 0.998 & 13 & 18 & 27 & 4 \\
\hline ZB & 0.684 & 22.043 & 0.000 & 0.000 & 0.000 & 0.000 & 0.121 & 0.005 & 0.106 & 12 & 18 & 27 & 4 \\
\hline BZP & 0.814 & 11.660 & 0.000 & 0.009 & 0.000 & 0.003 & 0.105 & 0.386 & 0.987 & 14 & 18 & 27 & 4 \\
\hline LIB & 0.564 & 2.975 & 0.039 & - & - & - & - & - & - & 14 & 18 & 27 & 4 \\
\hline OFL & 0.823 & 25.948 & 0.000 & 0.000 & 0.000 & 0.000 & 0.006 & 0.051 & 0.871 & 14 & 18 & 27 & 4 \\
\hline PL & 0.362 & 23.093 & 0.000 & 0.000 & 0.000 & 0.000 & 0.070 & 0.655 & 0.996 & 13 & 18 & 27 & 4 \\
\hline LPB & 0.998 & 10.128 & 0.000 & 0.000 & 0.000 & 0.005 & 0.897 & 0.848 & 0.971 & 13 & 18 & 27 & 4 \\
\hline BBP & 0.359 & 14.776 & 0.000 & 0.004 & 0.000 & 0.000 & 0.276 & 0.008 & 0.92 & 14 & 18 & 27 & 4 \\
\hline $\mathrm{HBC}$ & 0.842 & 1.948 & 0.132 & - & - & - & - & - & - & 12 & 18 & 27 & 4 \\
\hline BBC & 0.242 & 7.838 & 0.000 & 0.000 & 0.000 & 0.079 & 0.941 & 0.981 & 1.000 & 13 & 18 & 27 & 4 \\
\hline IPB & 0.999 & 1.521 & 0.219 & - & - & - & - & - & - & 13 & 18 & 27 & 4 \\
\hline IPL & 0.687 & 1.355 & 0.266 & - & - & - & - & - & - & 13 & 18 & 27 & 4 \\
\hline$O C B$ & 0.226 & 6.572 & 0.001 & 0.015 & 0.001 & 0.010 & 0.839 & 0.559 & 0.817 & 12 & 18 & 26 & 4 \\
\hline
\end{tabular}


morphism in EEB's population of $O$. nigripes. In each agegroup only one variable was significantly dimorphic: BBC in juveniles, CZL in subadults and IPB in adults (Table 26).

Sexual dimorphism in 0 . nigripes had been investigated before; for example, Myers \& Carleton (1981) studied populations from three localities in Paraguay. Initially they tested for sexual variation in specimens from the locality with larger sample size (Parque Nacional de Ybycuí; $n=62$ ) and reported the presence of dimorphism in three out of six cranial variables examined. Later they analyzed specimens from three localities together and seven out of 20 variables showed significant results for sexual variation. The authors also analyzed the contributions of the sexual and ontogenetic variation to the overall variation. In their results, age differences contributed substantially and significantly to the total variation of two of six characters. The effect of sexual variation, however, is small and almost undetectable (Myers \& Carleton, 1981). More recently, Machado et al. (2011) also evaluated sexual dimorphism in O. nigripes and found evidence of it in only five of the 24 variables analyzed.

Remarks: The great phenotypic plasticity of $O$. nigripes makes it difficult to differentiate it from $O$. flavescens, as discussed previously. Several characteristics evaluated in EEB specimens present multiple states with different frequencies in the population. Among them, coloration of the ventral pelage, which varies from whitish gray to yellowish gray, particularly in specimens in AC2; older individuals, $\mathrm{AC} 3$ and $\mathrm{AC4}$, have pelage predominantly whitish gray.

In the cranium, there is great variation in the length of the incisive foramen with respect to the palate. In juveniles and subadults it extends beyond the anterior surface of $M 1$, entering the palatine bridge. In adults it does not reach the anterior surface of $M 1$, being restricted to the rostral region of the palate. The shape of the incisive foramen also varies greatly (Fig. 39), tending to with parallel margins in juveniles and subadults, whereas in adults it is drop-shaped; however, in all age classes there were individuals that deviated from this pattern, suggesting that this structure should not be used to identify O. nigripes in the EEB population, as proposed by Paresque (2010) for the species. Another characteristic that varies considerably is the number and size of the posterolateral palatine pits: they are small in juvenile individuals, often appearing as a single perforation in them, whereas in adults there are more than one, inserted in a large fossa; this modification of the posterolateral palatine pits was described as occurring in species of the tribe Oryzomyini by Weksler (2006), who proposed that the change from one simple small pit to conspicuous perforations - usually more than one foramen that could or not be recessed in fossae - is a common pattern in the ontogenetic variation of species of the tribe.

Field observations: A total of 105 specimens of 0 . nigripes were identified in the EEB samples. Of these, 19

Table 26. Descriptive statistics (mean \pm standard deviation, and sample size) of 25 cranio-dental measuremenst of young, subadult, and adult specimens of Oligoryzomys nigripes from EEB, separated by sex, and results of Student's t-test for sexual dimorphism and its value of significance. Statistically significant values $(P<0.05)$ are indicated in bold.

\begin{tabular}{|c|c|c|c|c|c|c|c|c|c|c|c|c|}
\hline & \multicolumn{4}{|c|}{ Young } & \multicolumn{4}{|c|}{ Subadults } & \multicolumn{4}{|c|}{ Adults } \\
\hline & Males & Females & $t$ & Sig. & Males & Females & $t$ & Sig. & Males & Females & $t$ & Sig. \\
\hline GSL & $21.81 \pm 0.81(9)$ & $22.01 \pm 0.49(4)$ & -0.453 & 0.659 & $23.33 \pm 0.86(14)$ & $22.82 \pm 0.53(4)$ & 1.104 & 0.286 & $24.29 \pm 0.78(16)$ & $24.02 \pm 0.62(15)$ & 1.087 & 0.286 \\
\hline CIL & $18.88 \pm 0.81(8)$ & $18.65 \pm 1.90(5)$ & 0.316 & 0.758 & $20.67 \pm 0.79(14)$ & $20.18 \pm 0.23(4)$ & 2.029 & 0.060 & $21.57 \pm 0.72(16)$ & $21.48 \pm 0.65(15)$ & 0.342 & 0.735 \\
\hline CZL & $15.05 \pm 0.68(8)$ & $15.44 \pm 0.45$ (4) & -1.030 & 0.327 & $16.33 \pm 0.57(14)$ & $15.86 \pm 0.15(4)$ & 2.768 & 0.014 & $16.87 \pm 0.59(16)$ & $16.81 \pm 0.45(15)$ & 0.316 & 0.754 \\
\hline LD & $5.12 \pm 0.29(9)$ & $5.02 \pm 0.58(5)$ & 0.451 & 0.660 & $5.62 \pm 0.30(14)$ & $5.47 \pm 0.17(4)$ & 0.944 & 0.359 & $5.96 \pm 0.27(16)$ & $5.93 \pm 0.19(15)$ & 0.321 & 0.751 \\
\hline LIF & $4.04 \pm 0.28(9)$ & $3.82 \pm 0.55(5)$ & 1.046 & 0.316 & $4.40 \pm 0.20(14)$ & $4.24 \pm 0.93(4)$ & 1.572 & 0.135 & $4.62 \pm 0.27(16)$ & $4.54 \pm 0.17(15)$ & 0.911 & 0.370 \\
\hline BIF & $1.68 \pm 0.19(9)$ & $1.68 \pm 0.14(5)$ & -0.055 & 0.957 & $1.81 \pm 0.11(14)$ & $1.77 \pm 0.15(4)$ & 0.641 & 0.531 & $1.90 \pm 0.15(16)$ & $1.84 \pm 0.13(15)$ & 1.083 & 0.288 \\
\hline LM & $3.32 \pm 0.12(8)$ & $3.37 \pm 0.14(4)$ & -0.706 & 0.496 & $3.42 \pm 0.10(14)$ & $3.47 \pm 0.13(4)$ & -0.807 & 0.432 & $3.37 \pm 0.13(16)$ & $3.40 \pm 0.10(15)$ & -0.787 & 0.438 \\
\hline BM1 & $0.94 \pm 0.04(9)$ & $0.95 \pm 0.07(5)$ & -0.272 & 0.796 & $0.96 \pm 0.02(14)$ & $0.97 \pm 0.03(4)$ & -0.431 & 0.672 & $0.97 \pm 0.05(16)$ & $0.97 \pm 0.04(15)$ & 0.093 & 0.927 \\
\hline ID & $1.09 \pm 0.10(9)$ & $1.04 \pm 0.20(5)$ & 0.690 & 0.503 & $1.23 \pm 0.09(14)$ & $1.19 \pm 0.05(4)$ & 0.714 & 0.486 & $1.32 \pm 0.11(16)$ & $1.30 \pm 0.04(15)$ & 0.442 & 0.663 \\
\hline IW & $1.11 \pm 0.07(9)$ & $1.08 \pm 0.16(5)$ & 0.476 & 0.643 & $1.15 \pm 0.04(14)$ & $1.13 \pm 0.05(4)$ & 0.677 & 0.508 & $1.27 \pm 0.10(16)$ & $1.26 \pm 0.07(15)$ & 0.344 & 0.734 \\
\hline LN & $7.84 \pm 0.54(9)$ & $7.69 \pm 0.38(4)$ & 0.486 & 0.637 & $8.58 \pm 0.50(14)$ & $8.37 \pm 0.23(4)$ & 0.811 & 0.429 & $9.20 \pm 0.41(16)$ & $9.00 \pm 0.34(15)$ & 1.541 & 0.134 \\
\hline BR & $3.11 \pm 0.15(9)$ & $3.43 \pm 0.39(4)$ & -2.206 & 0.050 & $3.31 \pm 0.26(14)$ & $3.17 \pm 0.16(4)$ & 0.994 & 0.335 & $3.31 \pm 0.21(16)$ & $3.20 \pm 0.20(15)$ & 1.424 & 0.165 \\
\hline RL & $7.34 \pm 0.37(9)$ & $7.25 \pm 0.23(4)$ & 0.435 & 0.672 & $8.00 \pm 0.56(14)$ & $7.76 \pm 0.22$ (4) & 0.807 & 0.432 & $8.48 \pm 0.32(16)$ & $8.36 \pm 0.29(15)$ & 1.112 & 0.275 \\
\hline ZB & $10.89 \pm 0.42(9)$ & $11.35 \pm 0.77(3)$ & -1.374 & 0.200 & $11.89 \pm 0.40(14)$ & $11.70 \pm 0.38(4)$ & 0.822 & 0.423 & $12.24 \pm 0.46(16)$ & $12.24 \pm 0.58(15)$ & -0.011 & 0.991 \\
\hline BZP & $1.83 \pm 0.11(9)$ & $1.91 \pm 0.31(5)$ & -0.722 & 0.484 & $2.09 \pm 0.19(14)$ & $2.03 \pm 0.21(4)$ & 0.522 & 0.609 & $2.19 \pm 0.17(16)$ & $2.22 \pm 0.17(15)$ & -0.396 & 0.695 \\
\hline LIB & $3.50 \pm 0.12(9)$ & $3.50 \pm 0.21(5)$ & -0.023 & 0.982 & $3.62 \pm 0.15(14)$ & $3.60 \pm 0.56(4)$ & 0.156 & 0.878 & $3.51 \pm 0.14(16)$ & $3.48 \pm 0.18(15)$ & 0.483 & 0.633 \\
\hline OFL & $7.43 \pm 0.30(9)$ & $7.17 \pm 0.58(5)$ & 1.090 & 0.297 & $7.93 \pm 0.24(14)$ & $7.68 \pm 0.49(4)$ & 1.498 & 0.154 & $8.23 \pm 0.28(16)$ & $8.22 \pm 0.23(15)$ & 0.148 & 0.883 \\
\hline PL & $8.71 \pm 0.30(8)$ & $8.46 \pm 0.78(5)$ & 0.829 & 0.425 & $9.44 \pm 0.35(14)$ & $9.16 \pm 0.13(4)$ & 1.525 & 0.147 & $9.74 \pm 0.36(16)$ & $9.57 \pm 0.26(15)$ & 1.455 & 0.156 \\
\hline LPB & $3.83 \pm 0.13(8)$ & $3.75 \pm 0.25(5)$ & 0.817 & 0.431 & $4.11 \pm 0.18(14)$ & $3.97 \pm 0.07(4)$ & 1.480 & 0.158 & $4.16 \pm 0.14(16)$ & $4.08 \pm 0.23(15)$ & 1.258 & 0.219 \\
\hline BBP & $2.00 \pm 0.19(9)$ & $2.11 \pm 0.18(5)$ & -1.062 & 0.309 & $2.20 \pm 0.12(14)$ & $2.21 \pm 0.10(4)$ & -0.152 & 0.881 & $2.29 \pm 0.14(16)$ & $2.30 \pm 0.09(15)$ & -0.408 & 0.686 \\
\hline HBC & $7.21 \pm 0.27(8)$ & $7.35 \pm 0.27(4)$ & -0.878 & 0.400 & $7.46 \pm 0.37(14)$ & $7.43 \pm 0.29(4)$ & 0.144 & 0.887 & $7.52 \pm 0.26(16)$ & $7.39 \pm 0.25(15)$ & 1.411 & 0.169 \\
\hline BBC & $10.48 \pm 0.25(9)$ & $10.86 \pm 0.20(4)$ & -2.690 & 0.021 & $11.04 \pm 0.22(14)$ & $11.13 \pm 0.39$ (4) & -0.575 & 0.573 & $11.10 \pm 0.28(16)$ & $10.92 \pm 0.31(15)$ & 1.672 & 0.105 \\
\hline IPB & $8.19 \pm 0.33(9)$ & $8.49 \pm 0.26(4)$ & -1.634 & 0.130 & $8.43 \pm 040(14)$ & $8.79 \pm 0.21(4)$ & -1.703 & 0.108 & $8.76 \pm 0.43(16)$ & $8.35 \pm 0.39(15)$ & 2.743 & 0.10 \\
\hline IPL & $2.40 \pm 0.27(9)$ & $2.60 \pm 0.19(4)$ & -1.324 & 0.212 & $2.63 \pm 0.28(14)$ & $2.74 \pm 0.41(4)$ & -0.621 & 0.543 & $2.57 \pm 0.24(16)$ & $2.55 \pm 0.25(15)$ & 0.289 & 0.775 \\
\hline OCB & $5.31 \pm 0.15(8)$ & $5.38 \pm 0.13(4)$ & -0.788 & 0.449 & $5.53 \pm 0.18(14)$ & $5.44 \pm 0.12(4)$ & 0.971 & 0.346 & $5.62 \pm 0.18(15)$ & $5.51 \pm 0.10(15)$ & 1.977 & 0.061 \\
\hline
\end{tabular}



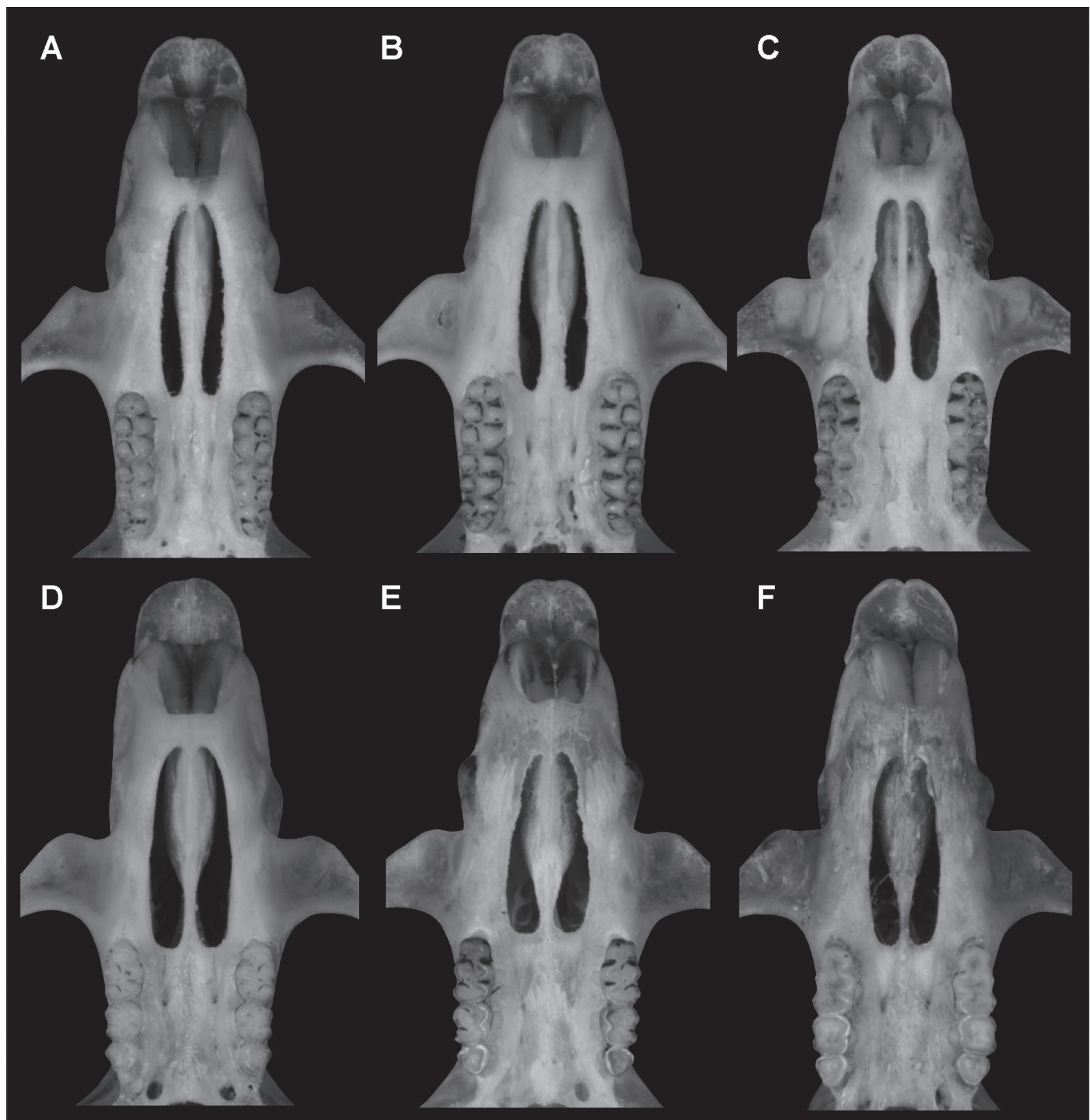

Figure 39. Variation of incisive foramina shape in specimens of Oligoryzomys nigripes in different age classes: (A) EBM $008(A C 2 ; \mathrm{LIF}=4.49 \mathrm{~mm})$; (B) EEB 660 (AC2; $\mathrm{LIF}=4.18 \mathrm{~mm}$ ); (C) EBM 047 (AC2; LIF = 4.22 mm); (D) EEB 839 (AC3; LIF = 5.29); (E) MZUSP 33730 (AC4; LIF=4.25 mm); (F) EEB 518 (AC5; LIF = 4.50 mm).

were collected in December, 2003, 69 in April, 2004, 11 in May, 2004, five in December, 2004, and one in December, 2011. Most captures (98.1\%) occurred in pitfall traps, and only two individuals were captured in conventional traps. The reproductive condition of specimens was as follows: in December, 2003, 11 males with scrotal testes and three females with perforated vaginas and/or mammary glands visible were captured, besides one male with abdominal testes and three females without signs of reproductive activity; reproductive information was not obtained for one specimen. In April, 2004, 10 males with scrotal testes, seven females with perforated vaginas and/or mammary glands visi- ble and 13 females without signs of reproductive activity were captured; information on the reproductive state of 39 specimens was not obtained. In December, 2001 a young male with abdominal testes was collected. For the remaining expeditions (May, 2004 and December, 2004) information on the reproductive state of specimens was not recorded.

Additional specimens examined: Oligoryzomys nigripes: $\mathrm{n}=5$; Brazil: São Paulo: Iperó: Floresta Nacional de Ipanema $\left(23^{\circ} 25^{\prime} 49^{\prime \prime} \mathrm{S}, 47^{\circ} 37^{\prime} 22^{\prime \prime} \mathrm{W}\right)$ : female: uncatalogued: APC 1781, 1834; male: uncatalogued: APC 1780, $1837,1856$. 
Table 27. Descriptive statistics (mean \pm standard deviation, range, and sample size) of body measurements $(\mathrm{mm})$, and weight ( $\mathrm{g}$ ), of adult specimens of Sooretamys angouya from EEB, and comparative descriptive statistics for the species obtained in Chiquito et al. (2014).

\begin{tabular}{l|cc|cc}
\hline & \multicolumn{2}{|c|}{ Females } & Males \\
\cline { 2 - 5 } & Species values & EEB specimens & Species values \\
\hline HBL & $166.98 \pm 17.91(125.0-215.0) 76$ & $153.80 \pm 20.60(125.0-173.0) 7$ & $163.74 \pm 16.93(126.0-198.0) 64$ \\
TL & $200.42 \pm 17.38(137.0-230.0) 77$ & $188.64 \pm 34.68(145.5-230.0) 7$ & $205.52 \pm 17.45(160.0-246.0) 64$ \\
HF1 & $33.71 \pm 3.56(20.0-39.0) 32$ & $33.13 \pm 2.36(30.0-37.0) 7$ & $35.66 \pm 2.37(31.5-40.0) 29$ & $139.80 \pm 7.07(127.0-145.0) 5$ \\
HF2 & $36.55 \pm 2.98(25.0-41.0) 80$ & $35.16 \pm 2.05(32.5-39.0) 7$ & $37.45 \pm 2.46(33.0-43.0) 67$ & $33.92 \pm 1.87(31.8-35.8) 5$ \\
EL & $22.30 \pm 2.86(13.0-26.5) 76$ & $21.10 \pm 1.37(19.6-22.5) 6$ & $22.75 \pm 2.56(16.0-34.0) 66$ & $35.54 \pm 2.10(32.2-37.5) 5$ \\
W & $114.44 \pm 30.93(56.0-220.0) 48$ & $83.90 \pm 39.50(43.0-132.0) 5$ & $117.70 \pm 43.02(64.0-280.0) 30$
\end{tabular}

\section{Sooretamys angouya (Fischer 1814)}

Voucher material: $\mathrm{n}=19$; female: MZUSP 33709; uncatalogued: EBM 063, 082, 095, 113, 142; EEB 646, 691, 727, 731; male: MZUSP 33708, 33710; uncatalogued: EBM 107, 120; EEB 742, 744, 768, 799; sex unknown: uncatalogued: EEB 907.

Specimens identification: Large size (see body measurements on Table 27). Dorsal pelage long and moderately dense, orangish/yellowish weakly grizzled with brown hairs; guard hairs long and moderately stiff (length ranging from 11 to $15 \mathrm{~mm}$ ), with half proximal portion thinner and grayish and distal portion wider and stiffer, black with tip brownish; cover hairs moderately long (length ranging from 8 to $12 \mathrm{~mm}$ ), with proximal three quarters grayish and distal quarter portion banded, with basal band orange and terminal band black; wool hairs very numerous, shorter, wavy and thin (length ranging from 6 to $9 \mathrm{~mm}$ ), with the basal portion grayish and apical portion $(1 / 10$ of the length of hairs) banded, with basal band orange and terminal band black. Lateral pelage similar to the dorsal pelage, predominantly brownish yellow, ochraceous yellow or rusty brown; transition with ventral region sharp, clearly delimited. Ventral pelage grayish-cream on abdominal and pectoral regions, with hairs with basal third grayish and distal two thirds cream, or cream on gular and inguinal regions with self-colored cream hairs; young individuals with ventral pelage predominantly grayish on abdominal and pectoral regions and whitish on gular and inguinal regions. Mystacial vibrissae long and moderately dense, reaching the tip of pinnae when laid backwards. Pinnae small and rounded densely covered internally and externally by short brown hairs. Manus short and moderately wide, covered dorsally by short brownish hairs; manus with long digits bearing short claws (claw of dlll with length ranging from 1.74 to $1.94 \mathrm{~mm}$; $\mathrm{n}=4$ ); dl vestigial, dll to dV longer, without conspicuous ungual tufts. Pes long and moderately wide, robust, with dorsal surface covered by short brown hairs, proximally, and grayish hairs distally; dlll longer than other digits; digits with long claws (claw of dlll with length ranging from 2.68 to $4.03 \mathrm{~mm} ; \mathrm{n}=4$ ); digits with sparse and long ungual tufts, not completely concealing the claws; pes with small interdigital pads; thenar and hypothenar pads present; plantar integument between pads covered by squamae; pads flesh colored. Tail longer than head and body $(125.3 \% \pm 12.5$ of head and body length, ranging from 104.8 to $145.4 \% ; n=16$ ); tail covered with short hairs, whose length equals the length of one to two scale rows on dorsal and ventral surface; apical tuft of hairs present, but small; scales large and arranged in 15 to 30 transverse rows per centimeter at the base and central portion of the tail, and between 19 to 32 rows on the apical centimeter of the tail $(n=4)$; tail brownish or grayish brown (skin and hairs) dorsally, and light brownish with some whitish hairs ventrally; apical tuft whitish.

Skull large and robust (Fig. 40; Table 28). Rostrum long and wide; rostral tube absent. Nasals very long, expanded laterally distally, with anterior margin projected beyond the superior incisors, and posterior margin (nasofrontal suture) surpassing (27.3\%) or not surpassing $(72.7 \%)$ the maxillar-frontal-lacrimal sutures $(n=11)$. Nasofrontal suture triangular (9.1\%) or rounded (90.9\%) $(n=11)$. Premaxillae not projected posteriorly, ending anteriorly (36.4\%, most adults), leveled (54.5\%, mostly young), or posteriorly (9.1\%, one adult) to the nasals $(n=11)$. Lacrimal equally in contact with the maxillar and frontal. Zygomatic notch very deep and wide. Interorbital region symmetrically constricted, narrower medially, with supraorbital margins squared $(72.7 \%)$ or slightly projected dorsally (beaded; $27.3 \%$ ), continuous with the sharp temporal margin $(n=11)$. Frontosquamosal suture collinear $(36.7 \%)$ or not $(63.6 \%)$ with the frontoparietal suture $(n=11)$. Zygomatic arches robust, projected laterally, wider at the squamosal root. Braincase narrow, with elongated profile. Parietals quite expanded ventrally, widely penetrating on the lateral surface of braincase $(90.0 \%)$ or restricted to the dorsal surface $(9.1 \%)$ ( $n=11$ ). Rostrum with gnathic process short; rostral tube absent. Zygomatic plate very wide, with anterior margin straight or slightly concave; free dorsal margin projected anteriorly, with rounded anterodorsal margin or with distinct dorsal acute process. Zygomatic arches wide, with jugal large with maxillar and squamosal processes not overlapping (63.6\%), or with jugal small with maxillar and squamosal processes overlapping, but not in contact $(36.4 \%)(n=11)$. Stapedial and sphenofrontal foramina, and squamosal-alisphenoid groove absent; large opening for the alisphenoid canal; presence of the anastomotic canal on the surface of alisphenoid (derived carotid and stapedial circulatory pattern 3; Voss, 1988). Alisphenoid strut absent, configuring oval accessory and buccinator-masticatory foramina confluent, on a single foramen oval. Postglenoid foramen present. Subsquamosal fenestra very small or absent. Stapedial 
Table 28. Descriptive statistics (mean \pm standard deviation, range, and sample size) of cranio-dental measurements (mm) of Sooretamys angouya from EEB, and comparative descriptive statistics for the species obtained in Abreu-Júnior et al. (2012).

\begin{tabular}{|c|c|c|c|c|c|}
\hline & & Females & & & Males \\
\hline & Species values & EEB adult specimens & EEB young and subadult specimens & Species values & EEB young and subadult specimens \\
\hline GSL & $39.26 \pm 1.60(35.01-43.83) 89$ & $39.44 \pm 0.96(38.04-40.13) 4$ & 34.06 & $39.62 \pm 2.21(32.87-44.31) 85$ & $33.59 \pm 2.34(29.68-35.74) 5$ \\
\hline CIL & $35.93 \pm 1.54(31.60-39.70) 94$ & $35.57 \pm 0.99(34.17-36.52) 4$ & 30.87 & $36.23 \pm 2.20(29.02-41.05) 92$ & $30.01 \pm 2.27(26.14-32.07) 5$ \\
\hline LD & $10.08 \pm 0.67(7.94-11.85) 102$ & $10.09 \pm 0.43(9.64-10.66) 4$ & $(6.99-8.44) 2$ & $10.22 \pm 0.79(8.18-12.10) 96$ & $8.34 \pm 0.64(7.40-8.89) 5$ \\
\hline LM & $6.00 \pm 0.25(5.41-6.70) 101$ & $6.14 \pm 0.22(5.86-6.32) 4$ & 6.01 & $6.09 \pm 0.27(5.16-6.76) 95$ & $5.83 \pm 0.26(5.53-6.12) 4$ \\
\hline BM1 & $1.68 \pm 0.08(1.49-1.90) 102$ & $1.74 \pm 0.07(1.65-1.80) 4$ & $(1.53-1.76) 2$ & $1.71 \pm 0.08(1.45-1.98) 95$ & $1.63 \pm 0.05(1.58-1.71) 5$ \\
\hline LIF & $8.17 \pm 0.50(6.71-9.46) 103$ & $8.09 \pm 0.20(7.87-8.32) 4$ & $(5.75-7.00) 2$ & $8.27 \pm 0.66(5.57-9.50) 96$ & $6.94 \pm 0.63(6.12-7.48) 5$ \\
\hline BIF & $2.86 \pm 0.21(2.39-3.35) 101$ & $2.84 \pm 0.13(2.73-3.02) 4$ & $(2.12-2.36) 2$ & $2.86 \pm 0.26(2.02-3.43) 93$ & $2.57 \pm 0.11(2.47-2.75) 5$ \\
\hline IPB & $10.81 \pm 0.83(8.24-13.14) 91$ & $10.50 \pm 0.57(9.81-11.16) 4$ & 8.82 & $10.80 \pm 0.79(8.02-12.35) 91$ & $9.04 \pm 0.95(7.89-10.19) 5$ \\
\hline IPL & $4.44 \pm 0.52(3.27-5.80) 92$ & $4.32 \pm 0.14(4.14-4.44) 4$ & 3.28 & $4.52 \pm 0.51(3.05-5.96) 90$ & $4.35 \pm 0.25(4.05-4.62) 5$ \\
\hline LN & $15.25 \pm 0.90(13.01-17.76) 93$ & $15.50 \pm 0.60(14.84-16.05) 4$ & 13.60 & $15.36 \pm 1.11(12.22-18.05) 90$ & $12.72 \pm 1.15(10.97-13.77) 5$ \\
\hline LPB & $6.93 \pm 0.51(5.40-7.94) 103$ & $7.16 \pm 0.56(6.76-7.81) 4$ & $(5.80-6.20) 2$ & $7.06 \pm 0.50(5.80-8.26) 96$ & $6.27 \pm 0.57(5.37-6.94) 5$ \\
\hline LIB & $5.41 \pm 0.24(4.90-6.00) 103$ & $5.52 \pm 0.32(5.05-5.76) 4$ & $(5.12-5.16) 2$ & $5.45 \pm 0.26(4.78-6.16) 96$ & $5.28 \pm 0.13(5.16-5.49) 5$ \\
\hline ZB & $19.95 \pm 0.93(17.00-21.99) 90$ & $19.96 \pm 0.90(19.31-21.25) 4$ & $(14.54-17.17) 2$ & $20.14 \pm 1.09(16.85-23.04) 89$ & $16.97 \pm 1.08(15.07-17.65) 5$ \\
\hline BZP & $4.38 \pm 0.37(3.56-5.42) 103$ & $4.36 \pm 0.15(4.17-4.49) 4$ & $(2.77-3.61) 2$ & $4.27 \pm 0.39(3.43-5.37) 96$ & $3.10 \pm 0.33(2.55-3.39) 5$ \\
\hline CZL & $27.96 \pm 1.1(24.52-30.59) 93$ & $27.88 \pm 0.62(27.14-28.57) 4$ & 24.47 & $28.23 \pm 1.69(22.34-31.93) 91$ & $23.75 \pm 1.75(20.78-25.33) 5$ \\
\hline OFL & $13.31 \pm 0.57(11.61-14.83) 102$ & $13.06 \pm 0.42(12.59-13.56) 4$ & $(10.14-11.93) 2$ & $13.48 \pm 0.77(10.79-14.97) 96$ & $11.46 \pm 0.83(10.05-12.06) 5$ \\
\hline
\end{tabular}

and dorsostapedial processes of ectotympanic present; external auditory meatus small and rounded. Mastoid perforated by small fenestra or completely ossified. Incisive foramina long and narrow, with lateral margins parallel or slightly wider medially and anterior and posterior margins acute; posterior margin not reaching the anterior alveoli of $\mathrm{M} 1$ (36.4\%, all adult specimens), reaching the alveolus of M1 (54.4\%) or penetrating between M1 $(9.1 \%)(n=11)$. Posterior margin of zygomatic plate positioned anteriorly to the alveoli of M1 (90.9\%) or on the same level of the alveoli of M1 (9.1\%) $(n=11)$. Palate wide and long, with central region deeper and with two deeper parallel depressions aligned with posterior palatine foramina (configuring a small medial crest on the posterior portion of the palate); palate with posterolateral palatal pits multiple, large and complex, recessed in deep palatal depressions $(81.8 \%)$ or pits multiple, large and complex, not positioned in deep depression (18.2\%, mostly young specimens). Mesopterygoid fossa not penetrating between molar series; roof of mestopterygoid fossa perforated by small sphenopalatine vacuities, anterior to the presphenoid/basisphenoid suture (80\%) or large sphenopalatine vacuities, along the presphenoid and basisphenoid (20\%) ( $n=11$ ). Parapterygoid fossa wider than $(40 \%)$ or with the same width $(60 \%)$ of the mesopterygoid fossa $(n=10)$. Auditory bullae small and moderately globose, with the periotic margin extending $(72.7 \%)$ or not $(27.3 \%)$ to the internal carotid canal $(n=11)$. Mandible very robust, with ramus deep (Fig. 40). Coronoid process wide, triangular or weakly falciform, much lower than the condyloid process; angular process rounded, slightly surpassing posteriorly the condyloid process; superior notch and inferior notch shallow. Capsular process of lower incisor present, as a discrete elevation (50\%, young specimens) or as a well-developed process (50\%, all adult specimens). Superior masseteric crest weakly developed, absent in few specimens (18.2\%) $(n=11)$, and inferior masseteric crests developed. Upper incisors opisthodont. Upper molar series parallel; molars with main cusps arranged in opposite pairs; molar series robust, long and wide; labial and lingual flexus moderately long and interpenetrating on medial plane of molar. M1 with procingulum divided by an enamel island (internal procingulum flexid; $63.3 \%$ ) or by the anteromedial flexus in two conules (9.1\%), or not divided (27.3\%); anteroloph well developed, fused to the labial portion of the antecone, defining a long anterofosset; mesoloph present, reaching the labial cingulum, fused to the mesostyle; paracone fused to the protocone medially and not by the median mure, forming a very long and oblique mesoflexus; with wear this flexus is closed labially by the fusion of the mesolophostyle to the paracone, forming a distinct, long and oblique mesofosset, that is bisected in two by an oblique paralophule near the labial margin of the fosset, originating two enamel islands (mesofossets: a smaller labial and a larger medial). $\mathrm{m} 1$ with procingulum not divided by the anteromedian flexid; anterolophid well developed, reaching the cingulid, and fused to the lingual portion of anterocone, defining a small anterofossetid; mesolophid well developed, fused to lingually to the entoconid, forming an entofossetid; protostylid and ectostylid small; ectostylid and ectolophid present.

Remarks: Comparing EEB specimens with the description of S. angouya by Weksler et al. (2006) and Chiquito et al. (2014) we noticed some discrepancies on morphological features. The ventral coloration according to Weksler et al. (2006) is entirely pale with the base of the hairs always gray, without patches (gular and inguinal) with unicolored hairs (cream), as observed in the EEB specimens; the presence of these patches was also not mentioned by Chiquito et al. (2014). In the cranial morphology, the posterior margin of the zygomatic plate, according to Weksler et al. (2006), is situated dorsally to the alveolus of M1, while in the EEB sample it is situated anteriorly in $90.9 \%$ of the specimens; another character 


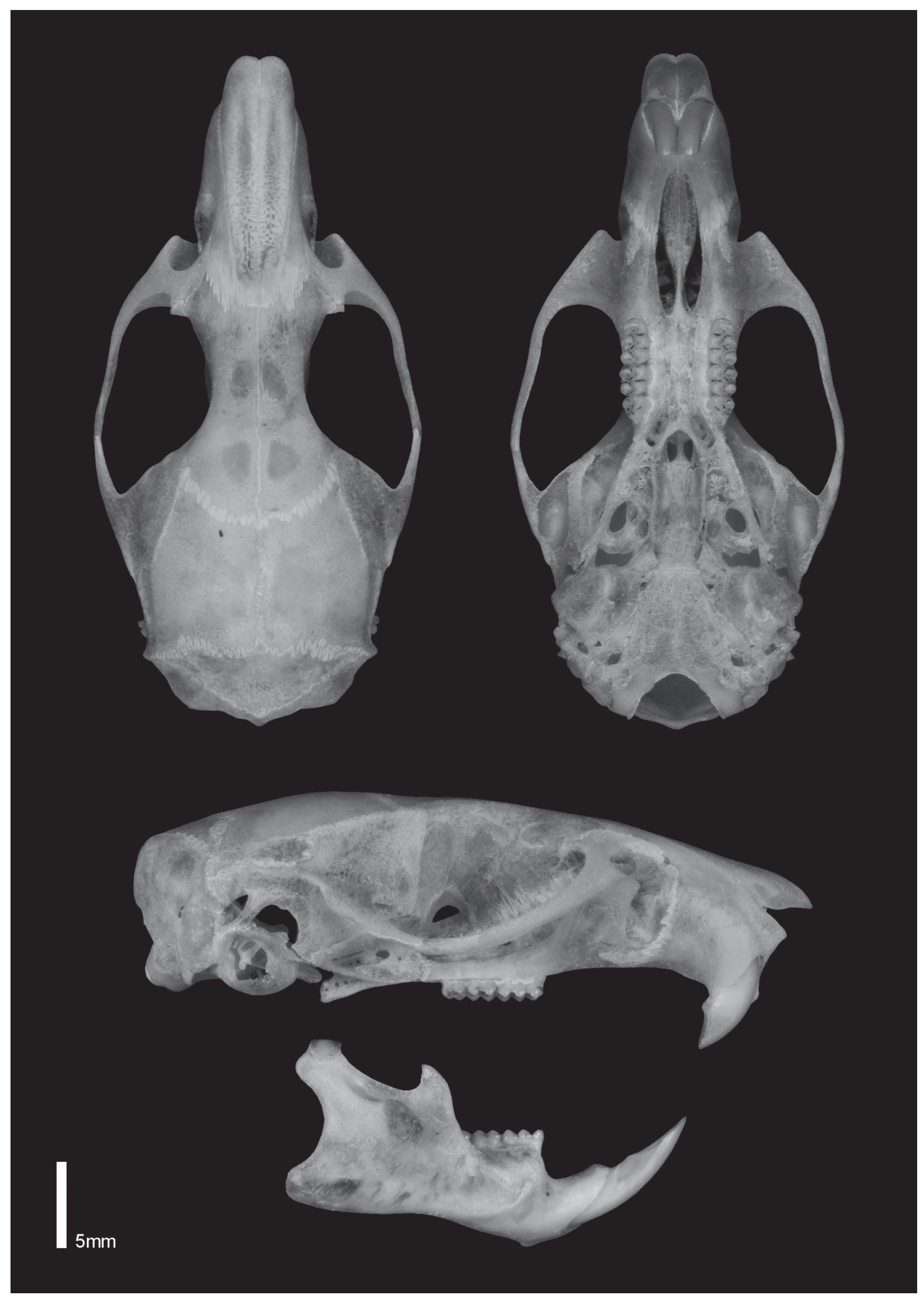

Figure 40. Skull and mandible of Sooretamys angouya (EBM 142; female; GSL =39.64 mm). Skull on dorsal, ventral, and lateral view, and mandible on lateral view. 
is the size of the posterior opening of the alisphenoid canal, which in the EEB specimens varies greatly, and it was described only as small by the authors above.

Field observations: Nineteen specimens of S. angouya were collected at EEB, 10 females, eight males and one specimen of undetermined sex. They were captured in 2003 and 2004 in different trails within EEB, using Sherman (10.5\% of the captures) and Pitfall (89.5\% of the captures) traps. Their reproductive condition was as follows: in December, 2003, six (out of 11) specimens were sexually active (i.e., males with scrotal testes and females with perforated vaginas or lactating) and three specimens did not show signs of reproductive activity (i.e., abdominal testes and females with unperforated vaginas); information about the reproductive condition of two specimens is unavailable. In April, 2004, four (out of seven) specimens did not show signs of reproductive activity, and the reproductive condition of the remaining specimens was not recorded.

\section{Tribe Thomasomyini Rhagomys rufescens (Thomas 1886)}

Voucher material: $\mathrm{n}=1$; male: uncatalogued: EEB 849 .
Specimen identification: Small size (see body measurements on Table 29). Dorsal pelage long and very dense, orangish/brown (rusty or rufous); guard hairs long (with about $9 \mathrm{~mm}$ in length), with half proximal portion thinner and grayish and distal portion wider dark brown; cover hairs moderately long (ca. $8 \mathrm{~mm}$ long), with proximal three quarters grayish and distal quarter portion orange; wool hairs very numerous, shorter, wavy and thin (with about $6 \mathrm{~mm}$ in length), with the basal portion grayish and apical portion ( $1 / 5$ of the length of hairs) banded, with basal band orange and terminal band black. Lateral pelage similar to the dorsal pelage, predominantly orangish (rufous); transition with ventral region subtle, not clearly delimited. Ventral pelage grayish-orange, with hairs with basal half or basal third grayish and distal portion orange. Mystacial vibrissae moderately long and dense, reaching the base of pinnae when laid backwards. Pinnae small and rounded, covered internally by short orange hairs arrange in a band near the border of pinnae and a band of brown hairs on the border; pinnae covered externally by short brown hairs. Manus short and moderately wide, covered dorsally by short grayish hairs; manus with long digits bearing short claws (claw of dIII = $1.2 \mathrm{~mm}$ in; $\mathrm{n}=1$ ); dl vestigial, dll to dV longer, without conspicuous ungual tufts. Pes short and moderately wide, delicate,

Table 29. Body and cranio-dental morphometric values $(\mathrm{mm})$, and weight $(\mathrm{g})$ of Rhagomys rufescens from Atlantic Forest localities. *Data from Pinheiro et al. (2004). ${ }^{* *}$ Specimens measured in this study. ${ }^{* * *}$ Data from Percequillo et al. (2004).

\begin{tabular}{|c|c|c|c|c|c|c|c|c|}
\hline & & Females & & & & & Sex unknow & \\
\hline & BMNH 86.2.8.5 (Holotype)* & MN $66056^{*}$ & MN 65545* & EEB 849** & CMUFLA 905** & MZUSP $31952^{* * *}$ & $A B 356^{* *}$ & AB 401** \\
\hline HBL & 94 & 75 & 107 & - & 65 & - & - & - \\
\hline TL & 93 & 94 & 112 & - & 105 & - & - & - \\
\hline HF2 & 20 & 19 & 21 & - & - & - & - & - \\
\hline HF1 & - & 18 & 21 & - & - & - & - & - \\
\hline EL & - & 12 & 15 & - & - & - & - & - \\
\hline w & - & 12 & 32 & - & 16 & - & - & - \\
\hline GSL & - & - & - & 26.65 & 26.41 & 26.24 & 23.68 & 28.50 \\
\hline CIL & - & 22.69 & 28.52 & 24.50 & 24.28 & - & 21.01 & 25.87 \\
\hline CZL & - & - & - & 19.77 & 19.70 & - & 16.99 & 20.75 \\
\hline LD & 7.90 & 6.02 & 8.20 & 7.34 & 7.21 & 7.02 & 5.82 & 7.41 \\
\hline LIF & 4.18 & 3.43 & 3.79 & 3.70 & 3.88 & 3.87 & 3.22 & 4.09 \\
\hline BIF & 1.65 & 1.34 & 1.56 & 1.38 & 1.15 & 1.34 & 1.35 & 1.64 \\
\hline LM & 4.76 & 4.59 & 4.56 & 4.17 & 4.26 & 4.39 & - & 4.65 \\
\hline BM1 & 1.31 & 1.29 & 1.29 & 1.12 & 1.18 & 1.23 & 1.14 & 1.24 \\
\hline ID & - & - & - & 1.51 & 1.56 & - & 0.98 & 1.74 \\
\hline IW & - & - & - & 1.54 & 1.68 & - & 1.38 & 1.76 \\
\hline LN & - & - & - & 9.39 & 8.32 & 8.30 & 7.09 & 9.39 \\
\hline BR & - & - & - & 3.11 & 3.12 & - & 3.30 & 3.70 \\
\hline RL & 10.15 & 8.10 & 11.37 & 9.19 & 8.73 & - & 7.08 & 9.79 \\
\hline ZB & - & 13.73 & 16.76 & 15.03 & 14.63 & - & - & 16.12 \\
\hline BZP & 3.72 & 3.23 & 4.35 & 3.09 & 3.18 & 3.08 & 2.32 & 3.38 \\
\hline LIB & 5.24 & 4.95 & 4.83 & 5.26 & 4.91 & 4.98 & 4.66 & 5.12 \\
\hline OFL & 10.75 & 9.72 & 10.95 & 9.89 & 10.02 & - & 8.99 & 10.52 \\
\hline PL & - & - & - & 11.96 & 11.63 & - & 10.50 & 12.94 \\
\hline LPB & 5.01 & 5.72 & 6.67 & 6.01 & 5.77 & - & 5.30 & 6.27 \\
\hline BBP & - & - & - & 2.59 & 2.42 & - & 2.02 & 2.43 \\
\hline HBC & - & - & - & 9.29 & 8.57 & - & 8.62 & 9.04 \\
\hline BBC & - & - & - & 13.27 & 12.28 & - & 12.14 & 13.57 \\
\hline IPB & - & - & - & 9.87 & 9.09 & - & 8.37 & 8.51 \\
\hline IPL & - & - & - & 4.55 & 4.06 & - & 3.89 & 4.12 \\
\hline OCB & - & 6.06 & 6.61 & 6.03 & 6.25 & - & 6.05 & 6.13 \\
\hline
\end{tabular}


with dorsal surface covered by short orange hairs; pes with dl short and with terminal pad enlarged and fleshy, dIII longer than dII and longer than dIV; digits with short claws (claw od dIII = $1.9 \mathrm{~mm} ; \mathrm{n}=1$ ); digits with sparse and long ungual tufts, not completely concealing the claws; ungual tufts with gray base and white apex; pes with moderately large interdigital pads; thenar and hypothenar pads present; plantar integument between pads covered by squamae; pads flesh colored. Tail longer than head and body length; tail covered by short hairs, whose length equals the length of two to three scale rows on dorsal and ventral surface; apical tuft of hairs present, but small, with $4.5 \mathrm{~mm}$; scales small and arranged in 20 rows per centimeter at the base of the tail, in 21 rows per $\mathrm{cm}$ on the central portion of the tail, and in 31 rows on the apical centimeter of the tail $(n=1)$; tail brownish dorsally (hairs entirely brown), brownish ventrally (hairs with brown base and white apex).

Skull small, short and moderately wide (Fig. 41; Table 29). Rostrum short and narrow; rostral tube absent. Nasals long, not expanded laterally distally, with anterior margin projected beyond incisors, and with posterior margin (nasofrontal suture) not surpassing the maxillar-frontal-lacrimal sutures. Nasofrontal suture triangular and acute. Premaxillae long, projected posteriorly beyond the nasals. Lacrimal equally in contact with the maxillar and frontal. Zygomatic notch very shallow and narrow. Interorbital region slightly divergent posteriorly, narrower anteriorly, with supraorbital margins rounded, continuous with the moderately sharp temporal margin. Frontosquamosal suture not collinear with the frontoparietal suture. Zygomatic arches delicate, projected laterally, wider at the squamosal root. Braincase large and voluminous, with rounded profile. Parietals restricted to the dorsal surface of skull. Interparietal long and wide. Rostrum with gnathic process very short; rostral tube absent. Zygomatic plate narrow, with anterior margin straight or very slightly concave; free dorsal margin barely projected anteriorly, with rounded and downward sloping anterodorsal margin. Zygomatic arches narrow, with jugal large with maxillar and squamosal processes not overlapping. Stapedial and sphenofrontal foramina, and squamosal-alisphenoid groove absent; large opening for the alisphenoid canal, and presence of the anastomotic canal on the surface of alisphenoid (derived carotid and stapedial circulatory pattern 3; Voss, 1988). Alisphenoid strut absent, configuring oval accessory and buccinator-masticatory foramina confluent, on a single foramen oval. Postglenoid foramen present. Subsquamosal fenestra very large. Stapedial process of ectotympanic present; external auditory meatus small and rounded. Mastoid perforated by small fenestra. Incisive foramina very short and very narrow, with lateral margins parallel and anterior margins acute and posterior margins rounded; posterior margin not reaching the anterior alveoli of M1. Posterior margin of zygomatic plate positioned anteriorly to the alveoli of M1. Palate wide and long, with central region deeper and with two deeper parallel depressions aligned with posterior palatine foramina (configuring a small medial crest on the posterior portion of the palate); palate with posterolateral palatal pits simple, not positioned in depressions; palatal excrescencies absent. Mesopterygoid fossa not penetrating between molar series; roof of mestopterygoid fossa perforated by small sphenopalatine vacuities, posteriorly to the presphenoid/basisphenoid suture. Parapterygoid fossa with the same width of the mesopterygoid fossa. Auditory bullae small and moderately globose, with the periotic margin extending to the internal carotid canal. Mandible with ramus low, and with lower border of the ramus completely straight (Fig. 41). Condyloid process robust and long. Coronoid process minute, almost not discernible on the dorsal surface of the ramus, weakly falciform, much lower than the condyloid process; angular process rounded, much shorter than condyloid process; superior notch undefined and inferior notch asymmetric and moderately deep. Capsular process of lower incisor absent. Superior and inferior masseteric crests weakly developed, converging in a V shape anteriorly. Upper incisors orthodont. Upper molar slightly convergent anteriorly; molar series delicate, short and narrow; molars with main cusps arranged in opposite pairs; main cusps high and isolated, not united by conspicuous enamel (or dentine) flexus; dentine exposition very small, restricted to the apical portion of the cusps; labial and lingual flexus incipient, not overlapping. M1 with procingulum divided by an asymmetric anteromedian flexus; labial anteroconule larger than lingual one; anteroloph absent; anterior mure present, as a small but distinct cone; mesoloph present, reaching the labial cingulum, fused to the mesostyle; paracone fused to the protocone by the median mure (median mure is a distinct, but small, cone on the molar topography), forming a very long and shallow paraflexus; mesoflexus very short; protostyle and enterostyle absent; posteroloph present, but small. $\mathrm{m} 1$ with procingulum not divided by the anteromedian flexid; anterolophid absent; mesolophid well developed, fused to lingually to the entoconid; protostylid and ectostylid small; ectostylid and ectolophid present.

Remarks: Despite information obtained in recent years (Pinheiro et al., 2004; Percequillo et al., 2004; SteinerSouza et al., 2008; Passamani et al., 2011), Rhagomys rufescens is one of the most elusive AF sigmodontine rodents. It was described by Thomas (1886) as Hesperomys rufescens, based on a female collected in the city of Rio de Janeiro. Thomas moved the species to a different genus in 1917, after he obtained a second specimen. Nearly a century later, Percequillo et al. (2004) recorded two additional specimens of $R$. rufescens from Minas Gerais and São Paulo states. Subsequently, as the result of the widespread use of pitfall traps (see Steiner-Souza et al., 2008 and also Passamani et al., 2011), specimens of $R$. rufescens have been sporadically collected, increasing the number of specimens available for study. However, the number of specimens in scientific collections is still not sufficient to precisely evaluate the morphologic and morphometric variation in the species.

In order to contribute to the knowledge about the morphology of this species, we compared the morpholo- 


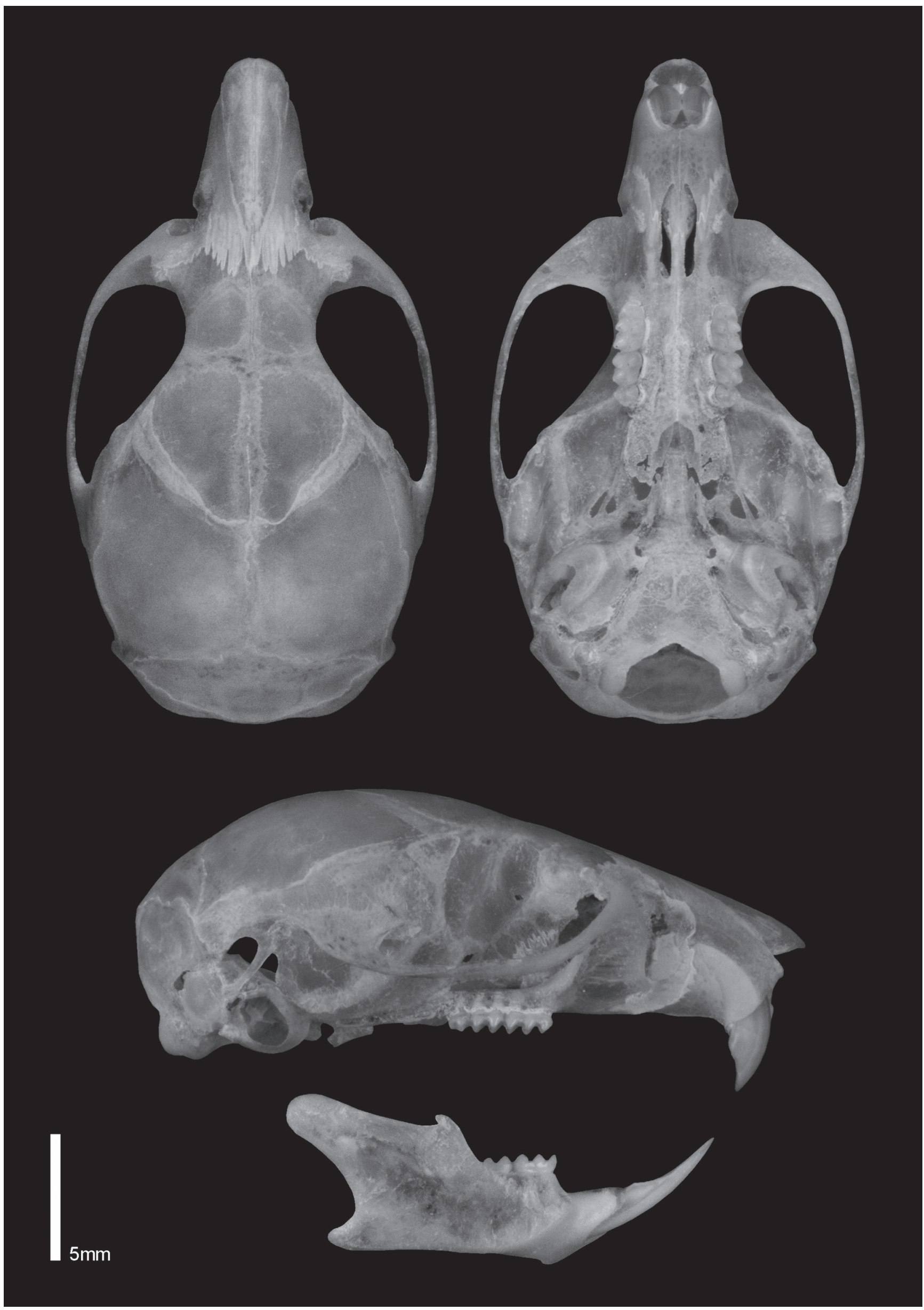

Figure 41. Skull and mandible of Rhagomys rufescens (EEB 849; male; GSL = $28.51 \mathrm{~mm}$ ). Skull on dorsal, ventral, and lateral view, and mandible on lateral view. 
gy of the EEB specimen with the descriptions provided by Percequillo et al. (2004) and Pinheiro et al. (2004). In the external morphology, the EEB specimen is very similar to those descriptions, with reddish brown dorsal coloration and buffy yellowish ventral coloration, similar tail length, vibrissae, manus and pes. In the cranial morphology, the specimen from EEB differs from others by the absence of alisphenoid strut; our specimen also lacks the stapedial foramen, which is consistent with the description of Percequillo et al. (2004), but contrasts with Pinheiro et al. (2004) - who described this structure as present and large; the latter authors also described a large post-orbital process, which was detected, though inconspicuous, in the EEB specimen. Percequillo et al. (2004) described the braincase as wide and elongated, whereas in the EEB specimen it has a rounder profile. In the molar morphology it is worth to mention the absence of the anteroloph of M1 in the EEB specimen; this structure has been described as being conspicuous in the species and was also observed by us in the specimens AB 356 and AB 401, from Ribeirão Grande, São Paulo.

Field observations: The specimen collected at EEB, a juvenile male, was captured with pitfall trap on May, 2004.

Additional specimens examined: Rhagomys rufescens: $\mathrm{n}=3$; Brazil: Minas Gerais: Parque Estadual da Serra do Papagaio (22 $\left.09^{\prime} \mathrm{S}, 44^{\circ} 43^{\prime} \mathrm{W}\right)$ : male: CMUFLA 905; São Paulo: Ribeirão Grande ( $\left.24^{\circ} 05^{\prime} 57^{\prime \prime} \mathrm{S}, 48^{\circ} 21^{\prime} 55^{\prime \prime} \mathrm{W}\right)$ : male: uncatalogued: $A B$ 356; female: uncatalogued: $A B 401$.

\section{Rhipidomys itoan Costa, Geise, Pereira \& Costa 2011}

Voucher material: $\mathrm{n}=17$; female: MZUSP 32525, 32526, 32527, 33716; uncatalogued: EEB 756, 795, 797; male: MZUSP 33715; uncatalogued: EBM 054, 064, 103, 201; EEB 769, 851, 852, 853; sex unknown: uncatalogued: EEB 892.

Specimens identification: Large size (see body measurements on Table 30). Dorsal pelage moderately long and dense, orangish/yellowish weakly to moderately grizzled with light brown hairs; guard hairs long and slightly stiff (length ranging from 10 to $11 \mathrm{~mm}$ ), with proximal half thinner and grayish and distal portion wider and stiffer, brownish; cover hairs moderately long (length ranging from 8.5 to $10 \mathrm{~mm}$ ), with proximal two thirds grayish and distal third portion copper; wool hairs very numerous, shorter, wavy and thin (length ranging from 5.5 to $7 \mathrm{~mm}$ ), with the basal portion grayish and apical portion ( $1 / 4$ of the length of hairs) banded, with basal band copper and terminal band brown. Lateral pelage light brown, grayish (due to the exposition of the gray bases of hairs); transition with ventral region sharp, clearly delimited. Ventral pelage white, with self-colored white hairs; in some specimens, in the transition between ventral and lateral regions, the abdominal region with hairs with basal third grayish and distal two white. Three pairs of mammary glands, in inguinal, abdominal and post-axial positions. Mystacial vibrissae very long and dense, surpassing the tip of pinnae when laid backwards. Pinnae small and rounded sparsely covered internally by whitish hairs and externally by short brown hairs. Manus short and moderately wide, covered dorsally by short brownish hairs and digits covered by whitish hairs; manus with long digits bearing short claws (claw of dIII with length ranging from 1.45 to $2.2 \mathrm{~mm} ; \mathrm{n}=3$ ); dl vestigial, dll to $\mathrm{dV}$ longer, without conspicuous ungual tufts. Pes long and wide, robust, with dorsal surface covered by short brown hairs, forming a distinct patch laterally; medially, pes are covered by whitish hairs; digits covered by white hairs, as the ungual tufts; pes with dIII similar to dIV, and longer than other digits; digits with long claws (claw of dIII with length ranging from 2.4 to $3.3 \mathrm{~mm} ; \mathrm{n}=3$ ); digits with sparse and long ungual tufts, not completely concealing the claws; pes with large and fleshy interdigital pads; thenar and hypothenar pads present; plantar integument between pads covered by squamae; pads flesh colored. Tail longer than head and body $(126.2 \% \pm 15.1$ of head and body length, ranging from 109.1 to $147.3 \% ; n=8$ ); tail covered by short hairs, whose length equals the length of two scale rows on dorsal and ventral surface; apical tuft of hairs present, but small; scales large and arranged in 13 to 15 transverse rows per centimeter at the base of the tail, between 14 to 17 rows per $\mathrm{cm}$ on the central portion of the tail, and between 18 to 20 rows on the apical centimeter of the tail $(n=3)$; apical tuft present, with 7 to $8 \mathrm{~mm}$; tail brownish dorsally, with hairs entirely brown; ventrally, basal portion of tail light brown (bicolored hairs, with brown base and white apex) and distal portion brown (brown hairs); apical tuft brown.

Skull robust, long and narrow (Fig. 42; Table 30). Rostrum moderately long and wide; rostral tube absent. Nasals long, expanded laterally distally, with anterior margin projected anteriorly beyond incisors, and posterior margin (nasofrontal suture) surpassing $(16.7 \%)$ or aligned $(83.3 \%)$ to the maxillar-frontal-lacrimal sutures ( $n=12$ ). Nasofrontal suture predominantly rounded $(91.7 \%)$ or triangular $(8.3 \%)(n=12)$. Premaxillae usually projected posteriorly, ending posteriorly (75\%) or at the same level $(25 \%)$ to the nasals $(n=12)$. Lacrimal equally in contact with the maxillar and frontal $(58.3 \%)$ or with the maxillar $(41.7 \%)(n=12)$. Zygomatic notch very shallow and narrow. Interorbital region symmetrically constricted, narrower medially, with supraorbital margins squared (66.7\%, mostly young and subadult specimens) or projected dorsally, forming beads or small crests (33.3\%, all adults), continuous with the sharp temporal margin $(n=12)$; temporal margin with a distinct, but small, process on the midportion of the parietals, posterior to the squamosal root of the zygomatic arch. Frontosquamosal suture collinear $(8.3 \%)$ or not $(91.7 \%)$ with the frontoparietal suture $(n=12)$. Zygomatic arches robust, projected laterally, slightly wider at the squamosal root. Braincase narrow, with elongated profile. Parietals quite expanded ventrally, widely penetrating on the lateral surface of braincase $(75 \%)$ or restricted to the dorsal surface $(25 \%)$ ( $n=12)$. Interparietal long and wide. Rostrum 
Table 30. Descriptive statistics (mean \pm standard deviation, range, and sample size) of body and cranio-dental measurements (mm), and weight ( $\mathrm{g}$ ), of Rhipidomys itoan from EEB, and descriptive statistics for the species obtained in Costa et al. (2011).

\begin{tabular}{|c|c|c|c|}
\hline & Species values & Young and subadult specimens from EEB & Adult specimens from EEB \\
\hline HBL & $136.37 \pm 13.35(119.0-165.0) 26$ & $108.04 \pm 9.41(95.0-117.2) 7$ & $130.67 \pm 19.22(110.0-148.0) 3$ \\
\hline TL & $173.85 \pm 19.41(138.0-212.0) 26$ & $134.89 \pm 16.98(105.0-151.0) 7$ & $165.00 \pm 6.08(161.0-172.0) 3$ \\
\hline HF2 & $29.42 \pm 3.24(18.5-33.5) 26$ & $27.36 \pm 1.29(25.0-28.7) 7$ & $29.17 \pm 1.76(27.5-31.0) 3$ \\
\hline HF1 & - & $26.11 \pm 1.33(24.0-27.7) 7$ & $27.50 \pm 1.50(26.0-29.0) 3$ \\
\hline EL & $18.58 \pm 2.83(12.0-22.0) 26$ & $17.09 \pm 1.43(15.0-19.0) 7$ & $18.83 \pm 0.29(18.5-19.0) 3$ \\
\hline w & $76.83 \pm 19.88(49.9-112.0) 20$ & $37.50 \pm 9.00(28.5-49.0) 7$ & $76.17 \pm 15.69(70.0-94.0) 3$ \\
\hline GSL & $34.43 \pm 1.67(31.72-37.46) 26$ & $30.43 \pm 1.13(28.68-31.61) 6$ & $33.97 \pm 1.07(32.54-35.47) 5$ \\
\hline CIL & $31.36 \pm 1.72(28.83-34.94) 27$ & $27.53 \pm 1.16(25.46-28.58) 6$ & $30.60 \pm 1.19(28.73-31.70) 5$ \\
\hline CZL & - & $21.68 \pm 0.98(20.00-22.60) 6$ & $23.94 \pm 1.05(22.39-25.27) 5$ \\
\hline LD & $8.76 \pm 0.69(7.53-10.37) 30$ & $7.57 \pm 0.49(6.75-8.13) 7$ & $8.85 \pm 0.28(8.62-9.30) 5$ \\
\hline LIF & $7.25 \pm 0.50(6.43-8.15) 30$ & $6.30 \pm 0.29(5.80-6.57) 7$ & $6.91 \pm 0.29(6.51-7.30) 5$ \\
\hline BIF & $2.74 \pm 0.25(2.32-3.27) 29$ & $2.74 \pm 0.18(2.55-3.01) 7$ & $2.92 \pm 0.13(2.72-3.08) 5$ \\
\hline LM & $5.13 \pm 0.10(4.94-5.33) 29$ & $5.16 \pm 0.14(4.93-5.34) 7$ & $5.13 \pm 0.24(4.89-5.49) 5$ \\
\hline BM1 & $1.41 \pm 0.04(1.34-1.48) 29$ & $1.37 \pm 0.05(1.31-1.44) 7$ & $1.41 \pm 0.06(1.36-1.51) 5$ \\
\hline ID & - & $1.52 \pm 0.08(1.43-1.62) 7$ & $1.80 \pm 0.08(1.67-1.86) 5$ \\
\hline IW & $2.11 \pm 0.11(1.92-2.30) 27$ & $1.71 \pm 0.11(1.55-1.83) 7$ & $2.02 \pm 0.14(1.85-2.18) 5$ \\
\hline LN & $11.51 \pm 0.92(9.81-13.30) 26$ & $10.14 \pm 0.75(9.13-11.16) 6$ & $11.28 \pm 0.25(11.01-11.58) 5$ \\
\hline BR & - & $4.14 \pm 0.15(3.85-4.36) 7$ & $4.55 \pm 0.29(4.18-4.91) 5$ \\
\hline $\mathbf{R L}$ & $10.65 \pm 0.75(9.52-12.31) 26$ & $9.68 \pm 0.74(9.24-10.36) 7$ & $11.54 \pm 0.25(11.18-11.83) 5$ \\
\hline ZB & $18.23 \pm 0.81(17.02-19.97) 26$ & $15.76 \pm 0.87(15.17-17.03) 7$ & $17.73 \pm 0.67(16.76-18.57) 5$ \\
\hline BZP & $2.79 \pm 0.22(2.44-3.42) 29$ & $2.30 \pm 0.27(1.98-2.63) 7$ & $2.70 \pm 0.25(2.36-3.04) 5$ \\
\hline LIB & $5.57 \pm 0.43(5.09-7.20) 30$ & $5.10 \pm 0.12(4.89-5.20) 7$ & $5.48 \pm 0.19(5.30-5.78) 5$ \\
\hline OFL & - & $10.37 \pm 0.44(9.67-10.89) 7$ & $11.59 \pm 0.65(10.97-12.56) 5$ \\
\hline PL & - & $12.42 \pm 0.57(11.30-13.00) 7$ & $13.63 \pm 0.56(12.91-14.38) 5$ \\
\hline LPB & $5.20 \pm 0.27(4.65-5.63) 30$ & $4.84 \pm 0.26(4.51-5.22) 7$ & $5.35 \pm 0.39(4.93-6.00) 5$ \\
\hline BBP & $3.29 \pm 0.20(2.74-3.67) 29$ & $3.21 \pm 0.09(3.11-3.37) 7$ & $3.38 \pm 0.14(3.24-3.57) 5$ \\
\hline HBC & - & $9.34 \pm 0.33(8.93-9.82) 6$ & $10.07 \pm 0.39(9.52-10.41) 5$ \\
\hline BBC & - & $14.02 \pm 0.48(13.35-14.68) 7$ & $14.94 \pm 0.59(14.47-15.92) 5$ \\
\hline IPB & - & $9.75 \pm 0.44(9.11-10.11) 6$ & $9.90 \pm 0.56(9.04-10.41) 5$ \\
\hline IPL & - & $4.59 \pm 0.15(4.41-4.81) 6$ & $5.11 \pm 0.30(4.75-5.57) 5$ \\
\hline OCB & - & $7.22 \pm 0.13(7.00-7.34) 6$ & $7.69 \pm 0.45(7.25-8.17) 5$ \\
\hline
\end{tabular}

with gnathic process short. Zygomatic plate very narrow, with anterior margin straight or slightly concave; free dorsal margin barely projected anteriorly, with rounded anterodorsal margin. Zygomatic arches wide, with jugal large with maxillar and squamosal processes not overlapping. Stapedial and sphenofrontal foramina, and squamosal-alisphenoid groove absent; large opening for the alisphenoid canal; presence of the anastomotic canal on the surface of alisphenoid (derived carotid and stapedial circulatory pattern 3; Voss, 1988). Alisphenoid strut present, configuring separate oval accessory and buccinator-masticatory foramina. Tegmen tympani well developed, overlapped to the suspensory process of squamosal, configuring a very small postglenoid foramen. Subsquamosal fenestra small. Stapedial and dorsostapedial processes of ectotympanic present; external auditory meatus small and rounded. Mastoid perforated by small fenestra or completely ossified. Incisive foramina long and wide, with lateral margins parallel or wider medially (convex) and anterior and posterior margins acute or rounded; posterior margin not reaching the anterior alveoli of $\mathrm{M} 1$ (41.7\%, all adult specimens), reaching the alveolus of $\mathrm{M} 1$ (33.3\%, subadult specimens) or penetrating between M1 (25\%, young specimens) ( $n=12)$. Posterior margin of zygomatic plate positioned anterior- ly to the alveoli of M1. Palate wide and short, with central region deeper and with two deeper parallel depressions aligned with posterior palatine foramina (configuring a small medial crest on the posterior portion of the palate); palate with posterolateral palatal pits small and single (83.3\%), or single and double in different sides of the skull $(16.7 \%)$, at palate level $(n=12)$. Mesopterygoid fossa reaching $(33.3 \%)$ or penetrating $(66.7 \%)$ between molar series; roof of mestopterygoid fossa completely ossified, not perforated by sphenopalatine vacuities. Parapterygoid fossa wider than $(18.2 \%)$ or with the same width $(81.8 \%)$ of the mesopterygoid fossa $(n=11)$. Auditory bullae small and moderately globose, with the periotic margin extending to the internal carotid canal $(66.7 \%)$ or with the periotic margin not contributing to the carotid canal (33.3\%) $(n=12)$. Mandible robust, with ramus deep (Fig. 42). Coronoid process wide, triangular or weakly falciform, lower than the condyloid process; angular process rounded, at the same level of the condyloid process; superior notch and inferior notch shallow. Capsular process of lower incisor present, as a discrete elevation. Crests weakly developed; superior crest present in some specimens $(41.7 \%)(n=12)$. Upper incisors opisthodont. Upper molar series slightly convergent anteriorly; molars with main cusps arranged in opposite pairs; 


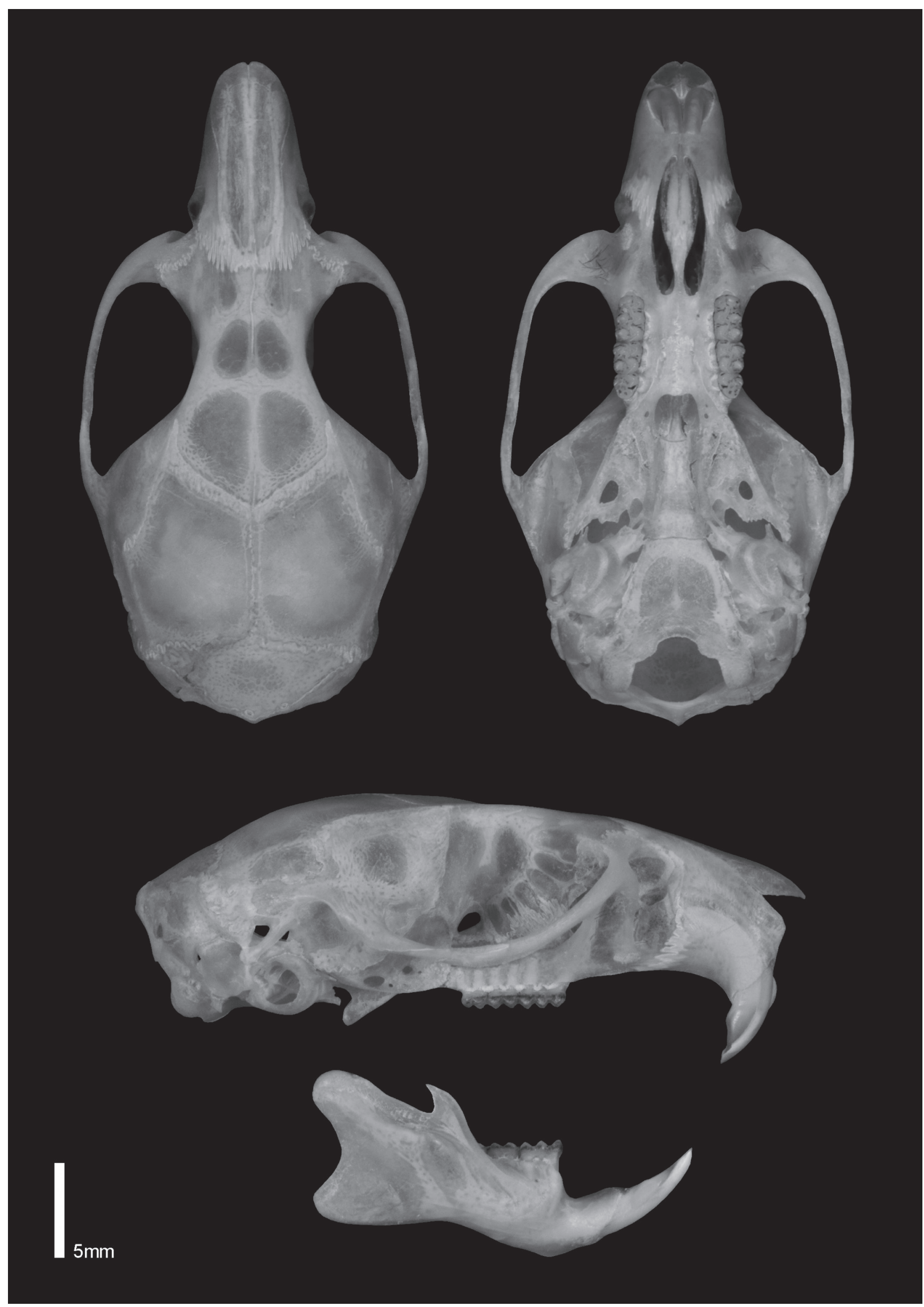

Figure 42. Skull and mandible of Rhipidomys itoan (EEB 851; male; GSL = $35.47 \mathrm{~mm}$ ). Skull on dorsal, ventral, and lateral view, and mandible on lateral view. 
molar series robust, long and wide; labial and lingual flexus moderately long and moderately interpenetrating on medial plane of molar. M1 with procingulum divided by the anteromedial flexus in two conules; anteroloph well developed, fused to the anterosyle; protostyle present; mesoloph present, reaching the labial cingulum, fused to the mesostyle; paracone fused to the protocone by the median mure; paracone fused by a paralophule to the mesoloph, forming a distinct mesofosset (with some wear); enterostyle present and enteroloph present (with some wear). $\mathrm{m} 1$ with procingulum divided by the anteromedian flexid; anterolophid well developed, reaching the cingulid, and fused to the lingual portion of anterocone, defining a small anterofossetid; protostylid present; mesolophid well developed, fused to lingually to the entoconid, forming an entofossetid; ectostylid and ectolophid present.

Remarks: According to Costa et al. (2011) and Tribe (2015), R. itoan can be differentiated from the other Rhipidomys species from southeastern Brazil (i.e., R. tribei, $R$. mastacalis and R. macrurus) by: its larger size: the presence of a small lacrimal projection (elongated and expanded laterally in $R$. tribei and $R$. macrurus); the presence of hourglass interorbital region with supraorbital crests conspicuous (not so in the other species); and by the presence of the incisive foramen converging anteriorly with posterior margins parallel, slightly converging on posterior tip. In general, these characteristics were also observed in EEB specimens, except for the shape of the incisive foramen which presents anterior and posterior margins converging. Morphometrically, adult specimens from EEB had values similar to those described for the species (see Table 30).

Field observations: Seventeen specimens of $R$. itoan were collected at EEB, 12 with pitfall traps, three with museum special traps, and two were collected accidently near EEB's main building. In December, 2003, nine specimens were collected: seven females, three showing signs of reproductive activity (two with mammary glands visible and perforated vagina and one pregnant), two without signs of reproductive activity (unperforated vagina), and two for which reproductive info is not available; and two males with abdominal testes. In April, 2004, four males were captured, but the reproductive state was observed for only one, as abdominal testes. In June 2004, three additional males were collected, without information on their reproductive state. In December 2004, the last specimen was captured, also without information on its reproductive state.

\section{Tribe Wiedomyini Genus Juliomys}

The genus Juliomys was described to accommodate Thomasomys pictipes Osgood - a species from eastern Brazil and northeastern Argentina (González, 2000). Subsequently, three new species were described for the genus: J. rimofrons (Oliveira \& Bonvicino, 2002), J. ossitenuis (Costa et al., 2007) and J. ximenezi (Christoff et al., 2016). Based on external and cranio-dental characteristics, we identified the specimens occurring at EEB as J. ossitenuis and J. pictipes. Externally, the major differences observed in specimens collected at EEB was in the coloration: the venter of J. pictipes is paler (whitish-buffy) than J. ossitenuis (ochraceous-buffy/grayish, with strong influence of the basal grayish portion of the hairs); and the color of the dorsum and the dorsal coloration of the hind feet of J. pictipes are more intensely orange than J. ossitenuis. Costa et al. (2007) reported an important difference in the color of the tail, strongly bicolor in J. pictipes versus slightly bicolor in J. ossitenuis. However, in specimens from EEB this character is very variable with age; the tails of juvenile individuals are frequently unicolor (dark on both surfaces) whereas in older specimens there is more distinction between the dorsal and ventral surfaces (dorsal dark and ventral pale), similar to what was observed in J. pictipes; the ontogenetic variation of this character needs to be considered for identification purposes.

The following characteristics of the cranial morphology appeared to be the most relevant to differentiate between the two species: the carotid and stapedial circulatory pattern, primitive in J. ossitenuis, J. rimofrons and J. ximenezi (pattern 1; Voss, 1988) and derived in J. pictipes (pattern 2; Voss, 1988), a difference that is uncommon in sigmodontines (but see Zygodontomys; Voss, 1991); the shape of the interorbital region, symmetrically constricted in J. ossitenuis and converging anteriorly in J. pictipes; sphenopalatine gaps, usually large in J. ossitenuis and reduced in J. pictipes; size of ectotympanic bulla, relatively large in J. ossitenuis and small in J. pictipes. The mandible is also diagnostic, robust and with ramus high in J. pictipes and delicate and with ramus low in J. ossitenuis.

Morphometrically, Pavan \& Leite (2011) described J. pictipes as being larger than J. ossitenuis, both in the external morphology and cranio-dental morphology. The small sample of J. pictipes precluded us for a consistent comparison among EEB specimens.

\section{Juliomys ossitenuis Costa, Pavan, Leite \& Fagundes 2007}

Voucher specimens: $\mathrm{n}=34$; female: MZUSP 33738, 33739; uncatalogued: EBM 015, 034, 049, 077, 119, 184, 191, 204; EEB 570, 837, 848, 1010; male: MZUSP 33736, $33737,33741,33743,33747$; uncatalogued: EBM 040, 057, 124, 143, 196, 244, 247, 253, 333; EEB 802, 812; unknown sex: uncatalogued: EBM 128, 202; EEB 868, 899.

Specimens identification: Small size (see body measurements on Table 31). Dorsal pelage long and dense, brown grizzled with orange, more intensely on the rump; guard hairs long (with length ranging from 10 to $11 \mathrm{~mm}$ ), with half proximal portion thinner and grayish and distal portion wider and with a basal band dark gray and apical band brown; cover hairs moderately long (length ranging from 7.5 to $11 \mathrm{~mm}$ ), with proximal two thirds grayish 
Table 31. Descriptive statistics (mean \pm standard deviation, range, and sample size) of body and cranio-dental measurements (mm), and weight ( $\mathrm{g}$ ), of Juliomys ossitenuis from EEB.

\begin{tabular}{|c|c|c|c|}
\hline & Youngs ( 2 females and 6 males) & Subadults ( 7 females and 4 males) & Adults (1female and 3males) \\
\hline $\mathrm{HBL}$ & $71.89 \pm 2.95(68.0-76.0) 8$ & $78.58 \pm 4.21(74.0-86.0) 8$ & $(90.0-87.6) 2$ \\
\hline TL & $97.55 \pm 3.53(92.7-102.6) 8$ & $107.22 \pm 6.42(98.4-115.0) 9$ & $(108.3-110.0) 2$ \\
\hline HF1 & $18.68 \pm 0.91(17.0-20.0) 8$ & $19.41 \pm 1.98(17.5-24.0) 9$ & $(19.0-21.0) 2$ \\
\hline HF2 & $19.96 \pm 0.75(18.4-21.0) 8$ & $20.67 \pm 1.84(19.1-25.0) 9$ & $(20.0-22.0) 2$ \\
\hline EL & $13.74 \pm 1.14(12.0-15.0) 8$ & $14.91 \pm 1.84(13.0-19.0) 9$ & $(15.2-16.0) 2$ \\
\hline w & $11.38 \pm 1.18(10.5-13.0) 4$ & $14.75 \pm 2.72(10.0-18.0) 6$ & 24.0 \\
\hline GSL & $22.42 \pm 0.32(21.99-22.90) 7$ & $23.59 \pm 0.68(22.19-24.84) 11$ & $25.41 \pm 0.39(25.09-25.95) 4$ \\
\hline CIL & $19.53 \pm 0.44(18.67-20.17) 8$ & $20.86 \pm 0.72(19.26-21.92) 11$ & $22.61 \pm 0.67(22.06-23.54) 4$ \\
\hline CZL & $15.36 \pm 0.26(14.85-15.62) 8$ & $16.37 \pm 0.57(15.10-17.19) 11$ & $17.74 \pm 0.45(17.40-18.38) 4$ \\
\hline LD & $5.56 \pm 0.16(5.25-5.74) 7$ & $5.98 \pm 0.25(5.69-6.53) 11$ & $6.58 \pm 0.31(6.29-7.00) 4$ \\
\hline LIF & $4.13 \pm 0.17(3.85-4.33) 8$ & $4.54 \pm 0.24(3.97-4.89) 11$ & $4.77 \pm 0.21(4.55-5.04) 4$ \\
\hline BIF & $1.68 \pm 0.11(1.55-1.86) 8$ & $1.76 \pm 0.08(1.62-1.87) 11$ & $1.73 \pm 0.11(1.59-1.85) 4$ \\
\hline LM & $3.83 \pm 0.12(3.64-3.96) 7$ & $3.82 \pm 0.14(3.56-3.95) 11$ & $3.89 \pm 0.14(3.79-4.09) 4$ \\
\hline BM1 & $1.05 \pm 0.05(0.97-1.09) 7$ & $1.04 \pm 0.04(0.96-1.12) 11$ & $1.06 \pm 0.04(1.01-1.10) 4$ \\
\hline ID & $1.15 \pm 0.06(1.04-1.21) 8$ & $1.19 \pm 0.08(1.04-1.34) 11$ & $1.35 \pm 0.03(1.31-1.39) 4$ \\
\hline IW & $1.35 \pm 0.08(1.22-1.47) 8$ & $1.40 \pm 0.12(1.21-1.55) 11$ & $1.68 \pm 0.06(1.62-1.73) 4$ \\
\hline LN & $7.50 \pm 0.56(6.67-8.50) 7$ & $8.07 \pm 0.39(7.56-8.66) 11$ & $9.12 \vee 0.14(8.97-9.29) 4$ \\
\hline BR & $2.94 \pm 0.20(2.60-3.22) 8$ & $2.83 \pm 0.12(2.70-3.05) 11$ & $3.04 \pm 0.17(2.86-3.20) 4$ \\
\hline $\mathbf{R L}$ & $7.27 \pm 0.30(6.90-7.83) 7$ & $7.77 \pm 0.33(7.33-8.30) 11$ & $8.72 \pm 0.16(8.58-8.94) 4$ \\
\hline ZB & $11.37 \pm 0.60(10.56-12.32) 8$ & $12.3 \pm 0.42(11.65-12.93) 10$ & $13.31 \pm 0.49(12.66-13.83) 4$ \\
\hline BZP & $1.71 \pm 0.09(1.55-1.81) 8$ & $1.88 \pm 0.14(1.72-2.15) 11$ & $2.11 \pm 0.14(1.90-2.19) 4$ \\
\hline LIB & $3.65 \pm 0.12(3.52-3.90) 8$ & $3.61 \pm 0.15(3.41-3.87) 11$ & $3.78 \pm 0.11(3.63-3.87) 4$ \\
\hline OFL & $8.05 \pm 0.23(7.56-8.36) 8$ & $8.52 \pm 0.28(8.00-8.92) 11$ & $9.04 \pm 0.31(8.69-9.41) 4$ \\
\hline PL & $8.69 \pm 0.19(8.38-9.00) 8$ & $9.19 \pm 0.34(8.60-9.65) 11$ & $9.89 \pm 0.39(9.52-10.33) 4$ \\
\hline LPB & $3.42 \pm 0.12(3.31-3.59) 8$ & $3.55 \pm 0.17(3.33-3.85) 11$ & $3.88 \pm 0.15(3.74-4.01) 4$ \\
\hline BBP & $2.18 \pm 0.11(2.01-2.36) 7$ & $2.35 \pm 0.15(2.11-2.60) 10$ & $2.64 \pm 0.09(2.51-2.73) 4$ \\
\hline HBC & $7.70 \pm 0.31(7.17-8.17) 8$ & $7.81 \pm 0.34(6.98-8.28) 11$ & $8.18 \pm 0.19(7.95-8.33) 4$ \\
\hline BBC & $11.14 \pm 0.27(10.71-11.48) 8$ & $11.52 \pm 0.31(11.05-11.91) 11$ & $11.90 \pm 0.27(11.70-12.29) 4$ \\
\hline IPB & $8.29 \pm 0.36(7.55-8.71) 8$ & $8.29 \pm 0.62(7.34-9.13) 11$ & $8.91 \pm 0.15(8.74-9.10) 4$ \\
\hline IPL & $2.91 \pm 0.19(2.66-3.19) 8$ & $3.01 \pm 0.32(2.43-3.54) 11$ & $3.47 \pm 0.16(3.24-3.59) 4$ \\
\hline OCB & $5.35 \pm 0.22(5.10-5.73) 8$ & $5.51 \pm 0.15(5.22-5.75) 11$ & $5.68 \pm 0.13(5.57-5.83) 4$ \\
\hline
\end{tabular}

and distal portion with a basal band orangish; wool hairs numerous, shorter, wavy and thin (length ranging from 5.5 to $9 \mathrm{~mm}$ ), with the basal portion grayish and apical portion ( $1 / 5$ of the length of hairs) orangish. Lateral pelage brown more intensely grizzled with orange, more predominantly orange on the rump; muzzle with hairs entirely orange, in contrast with the head, similar to the remaining lateral region; transition with ventral region moderately defined. Ventral pelage buffy to ochraceous buffy, slightly grayish; hairs with basal third grayish and distal portion white. Mystacial vibrissae long, reaching the base and surpassing the tip of pinnae when laid backwards. Pinnae small and rounded, weakly covered internally and more densely covered externally, both by buffy orange hairs. Manus short and moderately wide, covered dorsally predominantly by short yellowish white hairs, but also by few hairs with brownish basal portion and yellowish white apical portion; manus with moderately long digits bearing very short claws (claw od dIII with length ranging from 1.3 to $1.4 \mathrm{~mm} ; \mathrm{n}=5$ ); dl vestigial, dll to $\mathrm{dV}$ longer, without conspicuous ungual tufts. Pes short and narrow, with dorsal surface covered by short orangish hairs, few of them with with basal portion brown; pes with dl shorter, dIII longer than dII and shorter than dIV; digits with short claws (claw of dIII with length ranging from 2.2 to $2.5 \mathrm{~mm} ; \mathrm{n}=5$ ); digits with dense long ungual tufts, concealing the claws; ungual tufts whitish, as well as digits; pes with moderately large interdigital pads; thenar and hypothenar pads present; plantar integument between pads covered by squamae; pads flesh colored; pes with. Tail longer than head and body $(135.4 \% \pm 7.5$ of head and body length, ranging from 123.3 to $148 \%$; $\mathrm{n}=27$ ); tail hirsute, covered by short hairs, whose length equals the length of two to three scale rows on dorsal and ventral surface; apical tuft of hairs small; scales very small and arranged in 22 to 23 transverse rows per centimeter at the base of the tail, and between 24 to 29 rows per $\mathrm{cm}$ on the central portion of the tail $(n=5)$; tail brownish dorsally (hairs entirely brown), light brownish ventrally in young individuals, and even more lighter in adult individuals; ventral surface of tail with discrete dark line throughout its length.

Skull small and delicate, slightly wide (Fig. 43; Table 31). Rostrum short and narrow; rostral tube absent. Nasals long, not expanded laterally distally, with anterior margin projected beyond incisors and posterior margin (nasofrontal suture) extending (47.6\%) or not $(52.4 \%)$ extending beyond the maxillar-frontal-lacrimal sutures $(n=21)$. Nasofrontal suture rounded $(81 \%)$ or acute $(19 \%)(n=21)$. Premaxillae long, with caudal margin leveled to the nasals (9.5\%) or extending beyond (90.5\%) the nasals ( $n=21$ ). Lacrimal equally in contact with the 


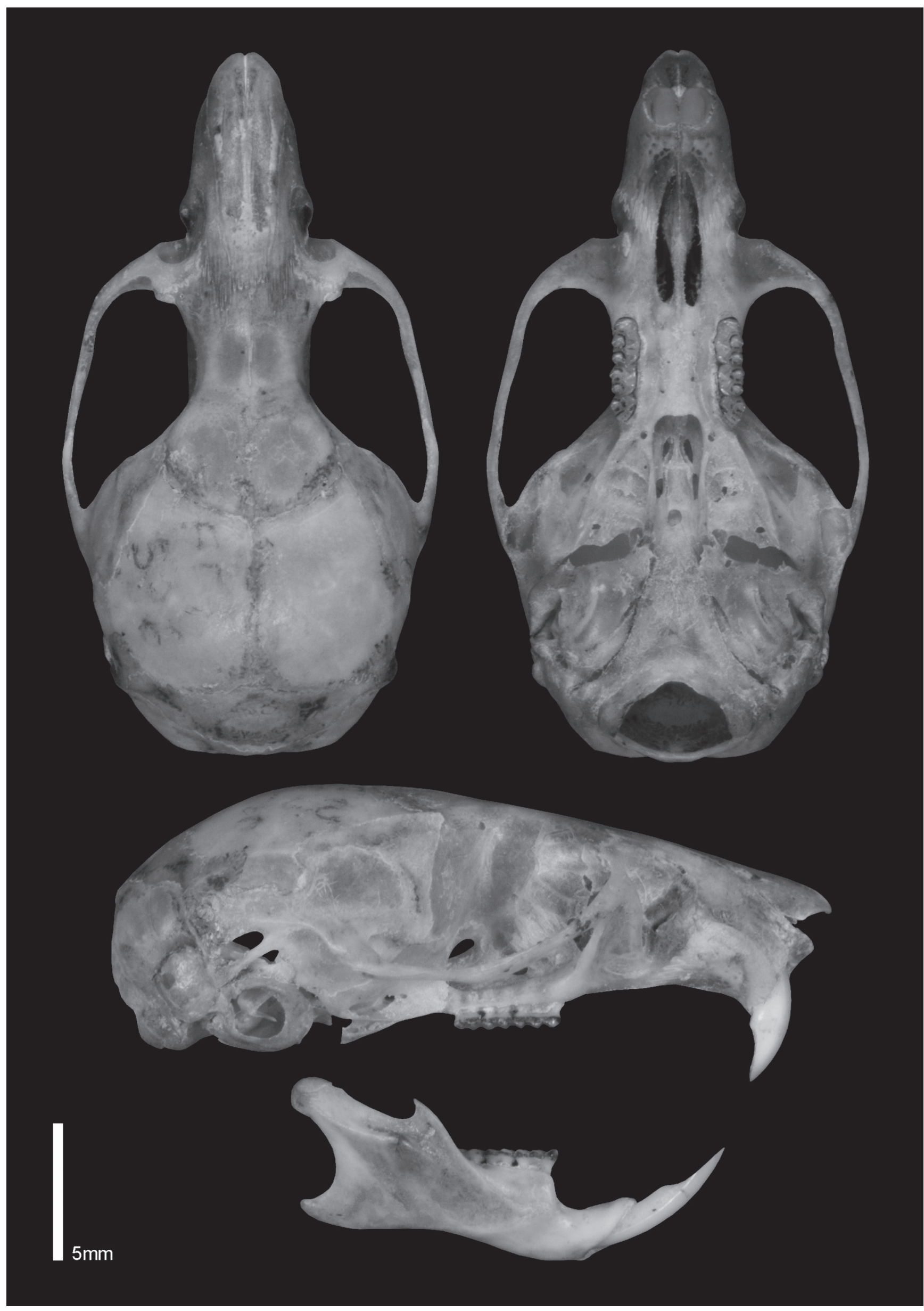

Figure 43. Skull and mandible of Juliomys ossitenuis (MZUSP 33736; male; GSL=25.95 mm). Skull on dorsal, ventral, and lateral view, and mandible on lateral view. 
maxillar and frontal (90.9\%) or only with the maxillar $(9.1 \%)(n=21)$. Zygomatic notch shallow. Interorbital region symmetrically constricted, with rounded $(39.1 \%)$ or with sharp, squared (60.9\%) supraorbital margins, continuous with the moderately sharp temporal margin ( $n=23)$. Interfrontal fontanelle absent $(78.3 \%)$ or present $(21.7 \%$; all specimens with the fontanelle are young and subadult) ( $n=23$ ). Frontosquamosal suture collinear (52.2\%) or not (47.8\%) with the frontoparietal suture $(n=23)$. Zygomatic arches delicate, projected laterally, wider at the squamosal root. Braincase small and slightly elongated. Parietals widely (68.2\%) or slightly (31.8\%) projected on the lateral surface of skull $(n=22)$. Rostrum with gnathic process very short. Zygomatic plate narrow, with anterior margin straight, slightly concave dorsally; free dorsal margin barely developed. Zygomatic arches moderate, with jugal large with maxillar and squamosal processes not overlapping. Stapedial foramen large; sphenofrontal foramen and squamosal-alisphenoid groove present; large opening for the alisphenoid canal (primitive carotid and stapedial circulatory pattern 1; Voss, 1988). Alisphenoid strut absent, configuring oval accessory and buccinator-masticatory foramina confluent on a single foramen oval (87\%) or present, with separated foramina (13\%) $(n=23)$. Suspensory squamosal process present and overlapped to the tegmen tympani, configuring a small postglenoid foramen. Subsquamosal fenestra very large. Stapedial process of ectotympanic present; external auditory meatus small and rounded. Mastoid perforated by small fenestra. Incisive foramina moderately long, with lateral margins divergent posteriorly; posterior margin reaching (34.8\%) or not (65.2\%) the anterior alveoli of M1 $(n=23)$. Posterior margin of zygomatic plate positioned much anterior to the alveoli of M1. Palate wide and short, with two shallow lateral depression aligned with posterior palatine foramina, deeper medially; palate with posterolateral palatal pits simple on the palate level (95.7\%) or as double perforations $(4.3 \%)$ at least in one sides of the skull $(n=23)$; palatal excrescencies absent. Mesopterygoid fossa penetrating between the molar series; roof of mesopterygoid fossa completely ossified $(8.7 \%)$ or perforated by small sphenopalatine vacuities, positioned posteriorly to the presphenoid-basisphenoid suture $(13 \%)$ or by large vacuities, along the presphenoid and basisphenoid (78.3\%) ( $n=23$ ). Parapterygoid fossa wider than $(95.7 \%)$ or with the same width (4.3\%) of the mesopterygoid fossa $(n=23)$. Auditory bullae small and moderately globose, with the periotic margin extending to the internal carotid canal. Mandible elongated, with ramus low (Fig. 43). Condyloid process large, projected posteriorly. Coronoid process small, triangular, much lower than the condyloid process; angular process moderately acute, slightly shorter than condyloid process; superior notch shallow and inferior notch moderately deep. Capsular process of lower incisor developed (60.9\%) or reduced, as a small elevation $(39.1 \%)(n=23)$. Superior masseteric crest well developed and inferior crest weakly developed, converging anteriorly. Upper incisors opisthodont. Upper molar series parallel; molar series small and delicate; molars with main cusps arranged in opposite pairs; main cusps moderately low, united by conspicuous enamel (or dentine) flexus; dentine exposition moderate; labial and lingual flexus conspicuous, moderately overlapping. M1 with procingulum divided by deep anteromedian flexus, very slightly asymmetric; labial anteroconule slightly larger than lingual one; anteroloph well developed; anteroflexus present; mesoloph robust, fused to mesostyle; mesoflexus short; protostyle present; enterostyle absent; posteroloph present. $\mathrm{m} 1$ with procingulum developed, divided by a deep the anteromedian flexid; anterolophid present; mesolophid developed; protostylid and protolophid present; small; ectostylid and ectolophid present.

Ontogenetic and sexual variation: The studied sample of J. ossitenuis from EEB is probably the largest series from a single locality, as less than 30 specimens from 15 localities were used in the original description of the species (Costa et al., 2007). The 23 EEB specimens with skulls available were subjected to age and sex variation analyses, the latter of which was conducted only on specimens in $\mathrm{AC2}$ ( $\mathrm{n}=11,7$ females and 4 males), considering the small sample sizes and the difference in the numbers of males and females in the remaining classes ( $A C 1, n=8,2$ females e 6 males; AC3, $n=4,1$ female and 3 males).

In the ontogenetic variation analysis, we compared values of 25 cranio-dental variables from specimens classified in three ACs. According to ANOVA, cranial divergence among the three classes was found; between individuals in $A C 1$ and $A C 2,13$ variables were statistically different (representing $52 \%$ of the variables analyzed), between individuals in $A C 1$ and $A C 3,18$ variables were significantly different (representing $72 \%$ of the variables analyzed), and between classes AC2 and AC3, 15 variables were statistically different (representing $60 \%$ of the variables analyzed) (Table 32; see also Table 31 for descriptive statistics). These results suggest that there are relevant cranial modifications associated with the ontogenetic development of $J$. ossitenuis, particularly in the length of the cranium, since out of the 18 variables with significant differences between at least two age classes, 11 are associated with the length of the cranium or the length of some structure (e.g., length of incisive foramen and length of interparietal), representing $61 \%$ of the variables with statistical differences. In practice, there is an increase in the cranial dimensions from the younger to the older class, and individuals in different age classes cannot be placed in the same category for analyses of sexual dimorphism and geographic variation, for example. In Costa et al. (2007) and in Pavan \& Leite (2011), the specimens of Juliomys classified in AC2 and AC3 based on the wear of the occlusion surface of the molars were considered adults and were clustered for descriptive statistics. Based on our results, this was an inaccurate interpretation of the age class variation of this species.

Some qualitative cranial characters also changed with age; for example: no adult individual examined exhibited the posterior margin of the incisive foramina reaching the anterior surface of $\mathrm{M} 1$; the parapterygoid fossa, in all adults, was excavated to some degree, dif- 
Table 32. Results of Kolmogorov-Smirnov test, Analysis of Variance (ANOVA), and Tukey's post-hoc test, and sample size in each age class for 25 cranio-dental morphometric variables measured in Juliomys ossitenuis from EEB. Statistically significant values $(\mathrm{P}<0.05)$ are indicated in bold.

\begin{tabular}{l|c|cc|ccc|ccc}
\hline & K-S & \multicolumn{2}{|c|}{ ANOVA } & \multicolumn{3}{|c|}{ Tukey } & \multicolumn{3}{c}{ N } \\
\cline { 2 - 10 } & Sig. & F & Sig. & $\mathbf{1}$ vs.2 & $\mathbf{1}$ vs. & $\mathbf{2}$ vs. 3 & $\mathbf{1}$ & $\mathbf{2}$ & $\mathbf{3}$ \\
\hline GSL & 0.652 & $\mathbf{3 8 . 4 8 0}$ & $\mathbf{0 . 0 0 0}$ & $\mathbf{0 . 0 0 1}$ & $\mathbf{0 . 0 0 0}$ & $\mathbf{0 . 0 0 0}$ & 7 & 11 & 4 \\
CIL & 0.962 & 33.045 & $\mathbf{0 . 0 0 0}$ & $\mathbf{0 . 0 0 1}$ & $\mathbf{0 . 0 0 0}$ & $\mathbf{0 . 0 0 0}$ & 8 & 11 & 4 \\
CZL & 0.757 & 35.346 & $\mathbf{0 . 0 0 0}$ & $\mathbf{0 . 0 0 0}$ & $\mathbf{0 . 0 0 0}$ & $\mathbf{0 . 0 0 0}$ & 8 & 11 & 4 \\
LD & 0.745 & $\mathbf{2 3 . 2 6 5}$ & $\mathbf{0 . 0 0 0}$ & $\mathbf{0 . 0 0 4}$ & $\mathbf{0 . 0 0 0}$ & $\mathbf{0 . 0 0 1}$ & 7 & 11 & 4 \\
LIF & 0.931 & $\mathbf{1 3 . 9 6 3}$ & $\mathbf{0 . 0 0 0}$ & $\mathbf{0 . 0 0 2}$ & $\mathbf{0 . 0 0 0}$ & 0.181 & 8 & 11 & 4 \\
BIF & 0.887 & 1.586 & 0.229 & - & - & - & 8 & 11 & 4 \\
LM & 0.770 & 0.417 & 0.665 & - & - & - & 7 & 11 & 4 \\
BM1 & 0.964 & 0.284 & 0.756 & - & - & - & 7 & 11 & 4 \\
ID & 0.779 & $\mathbf{1 1 . 9 7 2}$ & $\mathbf{0 . 0 0 0}$ & 0.364 & $\mathbf{0 . 0 0 0}$ & $\mathbf{0 . 0 0 2}$ & 8 & 11 & 4 \\
IW & 0.933 & $\mathbf{1 4 . 6 8 0}$ & $\mathbf{0 . 0 0 0}$ & 0.569 & $\mathbf{0 . 0 0 0}$ & $\mathbf{0 . 0 0 0}$ & 8 & 11 & 4 \\
LN & 0.855 & $\mathbf{1 8 . 3 3 6}$ & $\mathbf{0 . 0 0 0}$ & $\mathbf{0 . 0 3 1}$ & $\mathbf{0 . 0 0 0}$ & $\mathbf{0 . 0 0 1}$ & 7 & 11 & 4 \\
BR & 0.892 & 2.854 & 0.081 & - & - & - & 8 & 11 & 4 \\
RL & 0.575 & $\mathbf{3 0 . 2 7 0}$ & $\mathbf{0 . 0 0 0}$ & $\mathbf{0 . 0 0 6}$ & $\mathbf{0 . 0 0 0}$ & $\mathbf{0 . 0 0 0}$ & 7 & 11 & 4 \\
ZB & 0.996 & $\mathbf{2 0 . 7 3 8}$ & $\mathbf{0 . 0 0 0}$ & $\mathbf{0 . 0 0 3}$ & $\mathbf{0 . 0 0 0}$ & $\mathbf{0 . 0 0 8}$ & 8 & 11 & 4 \\
BZP & 0.559 & $\mathbf{1 4 . 3 2 5}$ & $\mathbf{0 . 0 0 0}$ & $\mathbf{0 . 0 2 0}$ & $\mathbf{0 . 0 0 0}$ & $\mathbf{0 . 0 1 2}$ & 8 & 11 & 4 \\
LIB & 0.769 & 2.486 & 0.109 & - & - & - & 8 & 11 & 4 \\
OFL & 0.728 & $\mathbf{1 8 . 3 8 7}$ & $\mathbf{0 . 0 0 0}$ & $\mathbf{0 . 0 0 4}$ & $\mathbf{0 . 0 0 0}$ & $\mathbf{0 . 0 0 9}$ & 8 & 11 & 4 \\
PL & 0.797 & $\mathbf{2 1 . 0 9 0}$ & $\mathbf{0 . 0 0 0}$ & $\mathbf{0 . 0 0 6}$ & $\mathbf{0 . 0 0 0}$ & $\mathbf{0 . 0 0 2}$ & 8 & 11 & 4 \\
LPB & 0.737 & $\mathbf{1 2 . 5 9 8}$ & $\mathbf{0 . 0 0 0}$ & 0.208 & $\mathbf{0 . 0 0 0}$ & $\mathbf{0 . 0 0 3}$ & 8 & 11 & 4 \\
BBP & 0.900 & $\mathbf{1 6 . 3 9 7}$ & $\mathbf{0 . 0 0 0}$ & $\mathbf{0 . 0 3 9}$ & $\mathbf{0 . 0 0 0}$ & $\mathbf{0 . 0 0 3}$ & 7 & 11 & 4 \\
HBC & 0.808 & 3.120 & 0.066 & - & - & - & 8 & 11 & 4 \\
BBC & 0.938 & $\mathbf{9 . 6 0 0}$ & $\mathbf{0 . 0 0 1}$ & $\mathbf{0 . 0 2 8}$ & $\mathbf{0 . 0 0 1}$ & 0.092 & 8 & 11 & 4 \\
IPB & 0.919 & 2.629 & 0.097 & - & - & - & 8 & 11 & 4 \\
IPL & 0.463 & $\mathbf{6 . 2 5 0}$ & $\mathbf{0 . 0 0 8}$ & 0.728 & $\mathbf{0 . 0 0 7}$ & $\mathbf{0 . 0 1 9}$ & 8 & 11 & 4 \\
OCB & 0.667 & $\mathbf{4 . 9 1 5}$ & $\mathbf{0 . 0 1 8}$ & 0.155 & $\mathbf{0 . 0 1 6}$ & 0.239 & 8 & 11 & 4 \\
\hline
\end{tabular}

fering from some juveniles, which had the structure at the same level as the palate; in the mandible, the capsular process of the alveoli of the lower incisors was always observed in adults, whereas in some juveniles this process was absent. Additionally, a relevant observation needs to be made about the interorbital fontanelle, a feature that was described as usually absent in J. ossitenuis (Costa et al., 2007). This trait appeared in moderately low frequencies in our sample $(21.7 \%)$, but we found that this trait appears to be influenced by age: the interorbital fontanelle is present only in juveniles and subadults, being absent in adult specimens.

Sexual dimorphism was evaluated in AC2 specimens, also from 25 cranio-dental variables, using the t-student test. The results in Table 33 demonstrate that sexual dimorphism is present in five of the 25 variables and that sexual differences are a consequence of the development of the anterior portion of the cranium: greatest skull length (GSL), length of incisive foramen (LIF), length of nasals ( $(L N)$, breadth of rostrum (BR) and rostral length (RL). The nature of the dimorphic variables is not surprising, since there is great variation in cranial-facial structures during the growth of many rodent species (e.g., Carleton \& Musser, 1989; Voss, 1991). Therefore, the probability of sexual differences in these variables is higher than in variables that vary little during development. Sexual selection and intraspecific niche divergence (Shine, 1989) are the most common sources of sexual dimorphism in mammals; there is a strong correlation between the location of these dimorphic variables and structures that are important to sexual behavior and resource foraging, such as those that enable hearing. However, the five dimorphic measurements represent $20 \%$ of the variables analyzed. Considering that sexual dimorphism, when present in sigmodontine rodents, is more accentuated in age-class 2 (see Hingst, 1995; Camardella et al., 1998; Libardi, 2013), it is possible that these differences do not represent a great source of variation in J. ossitenuis in this and older age classes, even though they have not been evaluated.

Remarks: As already mentioned, thirty specimens from 15 localities were used in the description of J. ossitenuis - which has made difficult to ascertain population variation within the species. Thirty-four specimens of J. ossitenuis were identified at EEB samples, 23 of which were used for qualitative and quantitative cranio-dental analyses, besides analyses of the external morphology of several of these specimens. In the cranial morphology, most characteristics observed in EEB specimens are consistent with the original description. Here, however, we provide the frequency of occurrence of polymorphic states; many characters are recognized to be variable (in Costa et al., 2007), but the frequency of their states was not known. One discrepancy with respect to the origi-

Table 33. Descriptive statistics (mean \pm standard deviation, and sample size) of 25 cranio-dental morphometric variables of subadult (AC2) specimens of Juliomys ossitenuis from EEB, separated by sex, and results of Student's t-test for sexual dimorphism and its value of significance. Statistically significant values $(P<0.05)$ are indicated in bold.

\begin{tabular}{|c|c|c|c|c|}
\hline & & Juliomys ossiten & & \\
\hline & Females & Males & $t$ & Sig. \\
\hline GSL & $23.27 \pm 0.52(7)$ & $24.16 \pm 0.56(4)$ & -2.635 & 0.027 \\
\hline CIL & $20.62 \pm 0.74(7)$ & $21.28 \pm 0.50(4)$ & -1.583 & 0.148 \\
\hline CZL & $16.14 \pm 0.53(7)$ & $16.78 \pm 0.42(4)$ & -2.052 & 0.070 \\
\hline LD & $5.88 \pm 0.17(7)$ & $6.17 \pm 0.29(4)$ & -2.190 & 0.056 \\
\hline LIF & $4.42 \pm 0.21(7)$ & $4.74 \pm 0.14(4)$ & -2.666 & 0.026 \\
\hline BIF & $1.76 \pm 0.07(7)$ & $1.76 \pm 0.10(4)$ & 0.047 & 0.963 \\
\hline LM & $3.81 \pm 0.15(7)$ & $3.82 \pm 0.13(4)$ & -0.061 & 0.952 \\
\hline BM1 & $1.03 \pm 0.02(7)$ & $1.05 \pm 0.07(4)$ & -0.456 & 0.677 \\
\hline ID & $1.18 \pm 0.10(7)$ & $1.21 \pm 0.03(4)$ & -0.549 & 0.596 \\
\hline IW & $1.39 \pm 0.15(7)$ & $1.42 \pm 0.06(4)$ & -0.530 & 0.610 \\
\hline LN & $7.87 \pm 0.21(7)$ & $8.43 \pm 0.40(4)$ & -3.143 & 0.012 \\
\hline BR & $2.78 \pm 0.09(7)$ & $2.92 \pm 0.11(4)$ & -2.324 & 0.045 \\
\hline $\mathbf{R L}$ & $7.62 \pm 0.27(7)$ & $8.04 \pm 0.27(4)$ & -2.452 & 0.037 \\
\hline ZB & $12.29 \pm 0.39(6)$ & $12.32 \pm 0.53(4)$ & -0.075 & 0.942 \\
\hline BZP & $1.86 \pm 0.15(7)$ & $1.92 \pm 0.13(4)$ & -0.667 & 0.521 \\
\hline LIB & $3.62 \pm 0.12(7)$ & $3.59 \pm 0.20(4)$ & 0.272 & 0.791 \\
\hline OFL & $8.44 \pm 0.28(7)$ & $8.65 \pm 0.26(4)$ & -1.229 & 0.250 \\
\hline PL & $9.09 \pm 0.37(7)$ & $9.35 \pm 0.23(4)$ & -1.224 & 0.252 \\
\hline LPB & $3.58 \pm 0.20(7)$ & $3.49 \pm 0.11(4)$ & 0.840 & 0.423 \\
\hline BBP & $2.39 \pm 0.15(6)$ & $2.29 \pm 0.13(4)$ & 1.035 & 0.331 \\
\hline HBC & $7.69 \pm 0.35(7)$ & $8.01 \pm 0.23(4)$ & -1.579 & 0.149 \\
\hline BBC & $11.45 \pm 0.29(7)$ & $11.64 \pm 0.35(4)$ & -0.960 & 0.362 \\
\hline IPB & $8.24 \pm 0.73(7)$ & $8.39 \pm 0.41(4)$ & -0.362 & 0.725 \\
\hline IPL & $2.89 \pm 0.36(7)$ & $3.21 \pm 0.10(4)$ & -1.735 & 0.117 \\
\hline$O C B$ & $5.51 \pm 0.13(7)$ & $5.52 \pm 0.20(4)$ & -0.120 & 0.907 \\
\hline
\end{tabular}


nal description is in the subesquamosal fenestra: it was originally described as being smaller than the post-glenoid foramen, but in all specimens evaluated by us it was larger.

In the external morphology, our observations are consistent with the species description (Costa et al., 2007), although we have found more variation in the color of the ventral and dorsal pelage: the ventral pelage, in some individuals, was slightly darker (tending to ochraceous-buffy) than the whitish-buffy coloration described for the species. This variation was more perceptible in juveniles; in the dorsal pelage, larger and older individuals had more intense orange coloration, and some juveniles had dorsal pelage predominantly brown and slightly grayish.

Field observations: During the sampling expeditions at EEB, nine specimens were collected in December, 2003, 20 in April, 2004, two in May, 2004, two in December, 2004, and one in December, 2011. Most were captured by pitfall traps (32 out of 34). In December, 2003, six males and three females were collected. Two of the males had scrotal testes and three did not, and there was no information about one specimen; one female had perforated vagina, whereas two presented unperforated vaginas. In April, 2004, 10 males and eight females were captured, besides two individuals of undetermined sex. The reproductive condition of none of the males was reported; seven of the eight females had unperforated vaginas; no reproductive activity was recorded in this period of the year. There was no reproductive information about the individuals collected on the other occasions.

\section{Juliomys pictipes (Osgood 1933)}

Voucher specimens: $\mathrm{n}=2$; female: uncatalogued: EBM 036; male: uncatalogued: EEB 885.

Specimens identification: Specimens with small size (see body measurements on Table 34). Dorsal pelage long and very dense, brown grizzled with orange; guard hairs long (with length ranging from 9 to $11.5 \mathrm{~mm}$ ), with half proximal portion thinner and grayish and distal portion wider and dark gray; cover hairs moderately long (length ranging from 7.7 to $8.5 \mathrm{~mm}$ ), with proximal four fifth grayish and distal portion with a basal band orangish and apical band black; wool hairs numerous, shorter, wavy and thin (length ranging from 6.4 to $6.8 \mathrm{~mm}$ ), with the basal portion grayish and apical portion $(1 / 5$ of the length of hairs) with basal band orangish and apical band black. Lateral pelage orange, brownish near the arms and more predominantly orange on the rump; muzzle with hairs entirely orange, in contrast with the head, similar to the remaining lateral region; transition with ventral region well defined. Ventral pelage buffy to whitish buffy, slightly grayish; hairs with basal third light gray and distal portion white, or self-colored white/whitish buffy hairs. Mystacial vibrissae long, reaching the base and surpassing the tip of pinnae when laid backwards. Pinnae small and rounded, densely covered internally and externally,
Table 34. Body and cranio-dental morphometric values $(\mathrm{mm})$, and weight $(\mathrm{g}$ ) of Juliomys pictipes from EEB.

\begin{tabular}{lcc}
\hline & EBM 036 (subadult, female) & EEB 885 (adult, male) \\
\hline HBL & 81.0 & - \\
TL & 99.6 & - \\
HF1 & 20.9 & - \\
HF2 & 21.4 & - \\
EL & 13.5 & - \\
W & 23.0 & - \\
GSL & 24.72 & 25.86 \\
CIL & 21.92 & 22.99 \\
CZL & 17.39 & 18.10 \\
LD & 6.01 & 6.51 \\
LIF & 4.27 & 4.72 \\
BIF & 1.71 & 1.82 \\
LM & 3.99 & 3.86 \\
BM1 & 1.05 & 1.08 \\
ID & 1.43 & 1.54 \\
IW & 1.65 & 1.73 \\
LN & 8.91 & 9.43 \\
BR & 3.22 & 3.34 \\
RL & 8.24 & 9.04 \\
ZB & 13.41 & 13.52 \\
BZP & 2.19 & 2.02 \\
LIB & 3.92 & 4.29 \\
OFL & 9.28 & 9.14 \\
PL & 9.72 & 10.19 \\
LPB & 4.20 & 4.15 \\
BBP & 2.35 & 2.26 \\
HBC & 7.85 & 7.91 \\
BBC & 12.15 & 11.90 \\
IPB & 9.26 & 9.43 \\
IPL & 3.74 & 3.41 \\
OCB & 6.14 & 6.00 \\
\hline & & \\
\hline & & \\
\hline
\end{tabular}

with orange and brown hairs, respectively. Manus short and moderately wide, covered dorsally predominantly by short orangish hairs; manus with moderately long digits bearing very short claws (claw of dIII with length ranging from 1.25 to $1.5 \mathrm{~mm} ; \mathrm{n}=2$ ); dl vestigial, $\mathrm{dll}$ to $\mathrm{dV}$ longer, without conspicuous ungual tufts. Pes short and narrow, with dorsal surface covered by short orangish hairs; pes with dl shorter, dIII similar in size to dII and dIV; digits with short claws (claw of dlll with about $2.2 \mathrm{~mm}$ in length; $\mathrm{n}=2$ ); digits with dense and long ungual tufts, concealing the claws; ungual tufts whitish, as well as digits; pes with moderately large interdigital pads; thenar and hypothenar pads present; plantar integument between pads covered by squamae; pads flesh colored. Tail longer than head and body (ca. 123\% of head and body length; $n=1$ ); tail hirsute, covered by short hairs, whose length equals the length of two to three scale rows on dorsal and ventral surface; apical tuft of hairs small; scales very small and arranged in 21 to 22 transverse rows per centimeter at the base of the tail, between 24 to 28 rows per $\mathrm{cm}$ on the central portion of the tail, and between 37 to 38 rows on the apical centimeter of the tail $(n=2)$; tail brownish dorsally (hairs entirely brown), light brownish ventrally (hairs whitish and orangish); ventral surface of tail with discrete dark line throughout its length. 
Skull small and delicate, slightly wide (Fig. 44; Table 34). Rostrum short and narrow; rostral tube absent. Nasals long, not expanded laterally distally, with anterior margin projected beyond incisors and posterior margin (nasofrontal suture) not extending beyond the maxillar-frontal-lacrimal sutures. Nasofrontal suture acute and triangular. Premaxillae short, with caudal margin leveled to the nasals. Lacrimal equally in contact with the maxillar and frontal. Zygomatic notch shallow. Interorbital region divergent posteriorly, with sharp, squared supraorbital margins, continuous with the moderately sharp temporal margin; interfrontal fontanelle absent. Frontosquamosal suture collinear with the frontoparietal suture. Zygomatic arches delicate, projected laterally, wider at the squamosal root. Braincase small and slightly elongated. Parietals predominantly restricted to the dorsal surface of skull. Rostrum with gnathic process very short. Zygomatic plate narrow, with anterior margin straight, slightly concave dorsally; free dorsal margin barely developed. Zygomatic arches moderate, with jugal large with maxillar and squamosal processes not overlapping. Stapedial foramen very large; sphenofrontal foramen and squamosal-alisphenoid groove absent; large opening for the alisphenoid canal (derived carotid and stapedial circulatory pattern 2; Voss, 1988). Alisphenoid strut absent, configuring oval accessory and buccinator-masticatory foramina confluent on a single foramen oval. Suspensory squamosal process present and overlapped to the tegmen tympani, configuring a small postglenoid foramen. Subsquamosal fenestra very large. Stapedial process of ectotympanic present; external auditory meatus small and rounded. Mastoid perforated by small fenestra. Incisive foramina moderately long, with lateral margins divergent posteriorly; posterior margin not reaching the anterior alveoli of M1. Posterior margin of zygomatic plate positioned much anterior to the alveoli of M1. Palate wide and short, with two shallow lateral depressions aligned with posterior palatine foramina, deeper medially; palate with posterolateral palatal pits simple on the palate level; palatal excrescencies absent. Mesopterygoid fossa penetrating between the molar series; roof of mestopterygoid fossa completely ossified or perforated by small sphenopalatine vacuities, positioned posteriorly to the presphenoid-basisphenoid suture. Parapterygoid fossa wider than the mesopterygoid fossa. Auditory bullae small and moderately globose, with the periotic margin extending to the internal carotid canal. Mandible elongated, with ramus low (Fig. 44). Condyloid process large, projected posteriorly. Coronoid process small, triangular, much lower than the condyloid process; angular process moderately acute, slightly shorter than condyloid process; superior notch shallow and inferior notch moderately deep. Capsular process of lower incisor developed. Superior masseteric crest well developed and inferior crest weakly developed, converging anteriorly. Upper incisors opisthodont. Upper molar parallel; molar series small and delicate; molars with main cusps arranged in opposite pairs; main cusps moderately low, united by conspicuous enamel (or dentine) flexus; dentine exposition moderate; labial and lingual flexus conspicuous, moderately overlapping. M1 with procingulum divided by deep anteromedian flexus, very slightly asymmetric; labial anteroconule slightly larger than lingual one; anteroloph well developed; anteroflexus present; mesoloph robust, fused to mesostyle; mesoflexus short; protostyle present; enterostyle absent; posteroloph present. $\mathrm{m} 1$ with procingulum developed, divided by a deep the anteromedian flexid; anterolophid present; mesolophid developed; protostylid and protolophid present; small; ectostylid and ectolophid present.

Field observations: Two specimens of J. pictipes were collected at EEB, the first in April, 2004, a female without signs of reproductive activity (unperforated vagina), and the second in December, 2004, a male without scrotal testes. Both were captured with pitfall traps.

\section{Phaenomys ferrugineus (Thomas 1894)}

\section{Voucher specimens: $\mathrm{n}=1$; male: uncatalogued: EBM 190.}

Specimen identification: Large size (see body measurements on Table 35). Dorsal pelage long and very dense, predominantly rusty-orange, with brown hairs along the mid-dorsal region, especially at the rump; guard hairs long (about $18 \mathrm{~mm}$ in length), with half proximal portion thinner and grayish and distal portion wider and orange-brown; cover hairs moderately long (with nearly $14 \mathrm{~mm}$ ), with proximal two thirds grayish and distal portion rusty-orange; wool hairs numerous, shorter, wavy and thin (about $10 \mathrm{~mm}$ in length), with the basal portion grayish and apical portion ( $1 / 4$ of the length of hairs) orangish. Lateral pelage rusty-orange, slightly lighter than the dorsum; transition with ventral region well defined. Ventral pelage buffy to whitish buffy, hairs with basal third light gray and distal portion white on thoracic and abdominal, or self-colored white/whitish buffy hairs on gular and inguinal regions. Mystacial vibrissae dense and long, reaching the base and surpassing the tip of pinnae when laid backwards. Pinnae small and rounded, densely covered internally and externally, with orange and brown hairs, respectively. Manus short and moderately wide, covered dorsally predominantly by short entirely white hairs and brown base hairs; manus with moderately long digits bearing very short claws (claw of dIII $=2.18 \mathrm{~mm}$; $\mathrm{n}=1$ ); dl vestigial, dll to dV longer, without conspicuous ungual tufts. Pes long and wide, with dorsal surface covered by long grayish brown and whitish hairs; pes with dl shorter, dIII similar in size to dII and dIV; digits with long claws (claw of dIII $=3.78 \mathrm{~mm} ; \mathrm{n}=1$ ); digits with moderately dense and long ungual tufts, with the same length and not concealing the claws; ungual tufts whitish, as well as digits; pes with large interdigital pads; thenar and hypothenar pads present; plantar integument between pads covered by squamae; pads flesh colored. Tail longer than head and body (ca. $136 \%$ of head and body length; $\mathrm{n}=1$ ); tail hirsute, covered by short hairs, whose length equals the length of two to three scale rows on dorsal and ventral surface; apical tuft of hairs small; scales very 


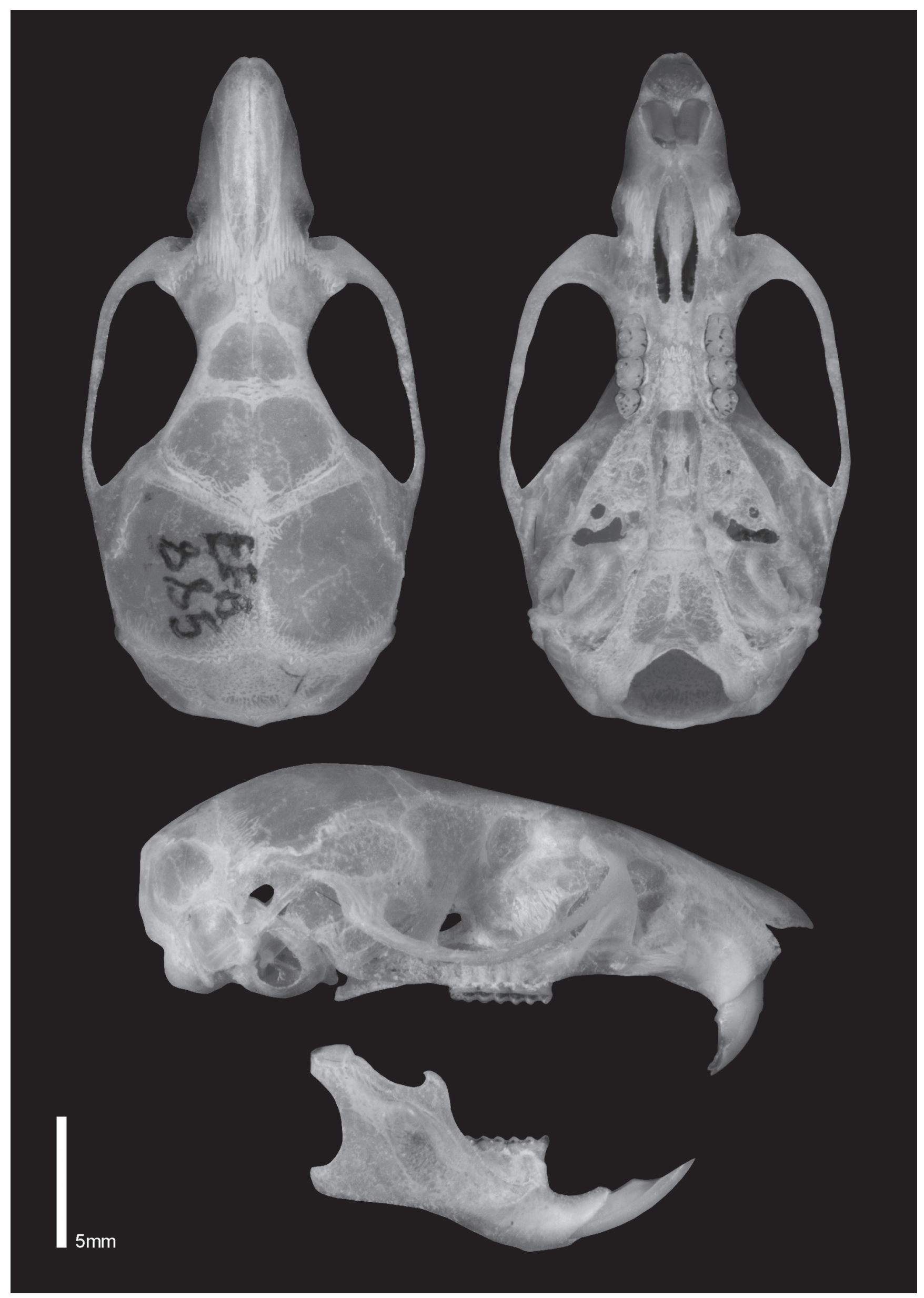

Figure 44. Skull and mandible of Juliomys pictipes (EEB 885; male; GSL = $25.86 \mathrm{~mm}$ ). Skull on dorsal, ventral, and lateral view, and mandible on lateral view. 
Table 35. Corporeal and cranio-dental morphometric values (mm), and weight (g) of Phaenomys ferrugineus. *Data from Bonvicino et al. (2001).

\begin{tabular}{|c|c|c|c|c|c|c|c|c|c|c|c|c|}
\hline & 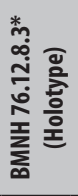 & 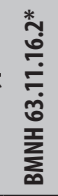 & 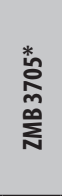 & $\begin{array}{l}\stackrel{*}{\stackrel{2}{\circ}} \\
\stackrel{m}{0} \\
\sum_{N}^{\infty}\end{array}$ & $\begin{array}{l}\text { \% } \\
\text { Oे } \\
\text { స్ } \\
\sum_{N}^{\infty}\end{array}$ & 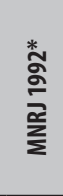 & 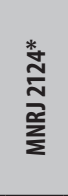 & 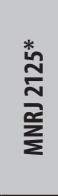 & 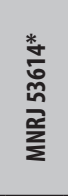 & 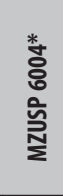 & 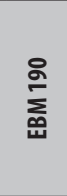 & 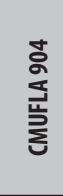 \\
\hline & - & female & male & female & - & - & male & female & male & male & male & male \\
\hline je class & - & - & - & - & - & - & - & - & - & - & 2 & 2 \\
\hline$A B L$ & 148.0 & - & 146.0 & 151.0 & 155.0 & 175.0 & 160.0 & 175.0 & 170.0 & 143.0 & 144.6 & 148.0 \\
\hline & 187.0 & - & 197.0 & 195.0 & - & - & 220.0 & 200.0 & 199.0 & 192.0 & 196.5 & 184.0 \\
\hline & & 31.0 & 32.0 & 32.0 & 30.0 & 30.0 & - & 30.0 & 29.0 & - & 32.6 & 31.0 \\
\hline & & - & 34.0 & 34.0 & 32.0 & 32.0 & - & 32.0 & 32.0 & 31.0 & 34.1 & 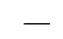 \\
\hline & 7.0 & 19.0 & - & 18.0 & - & - & - & - & 20.0 & - & 18.3 & 20.0 \\
\hline & - & - & - & - & - & - & - & - & - & - & 64.0 & 80.0 \\
\hline & - & - & - & - & - & 39.52 & 40.11 & - & 37.83 & - & 35.96 & 35.9 \\
\hline FII & - & - & - & - & - & 34.95 & 36.19 & - & 34.96 & - & 31.96 & 32.21 \\
\hline & - & - & - & - & - & - & - & - & - & - & 25.23 & 24.91 \\
\hline & - & - & - & - & - & 9.51 & 10.38 & - & 9.34 & - & 8.65 & 8.84 \\
\hline & - & - & - & - & - & 8.09 & 8.06 & - & 7.25 & - & 7.17 & 7.22 \\
\hline & - & - & - & - & - & 3.12 & 3.22 & - & 3.27 & - & 3.02 & 3.36 \\
\hline & - & - & - & - & - & 6.55 & 6.42 & - & 6.36 & - & 6.47 & 6.42 \\
\hline & - & - & - & - & - & - & - & - & - & - & 1.99 & 1.92 \\
\hline & - & - & - & - & - & - & - & - & - & - & 1.61 & 1.69 \\
\hline & - & - & - & - & - & - & - & - & - & - & 2.15 & 2.19 \\
\hline & - & - & - & - & - & - & - & - & - & - & 14.84 & 12.25 \\
\hline D & - & - & - & - & - & - & - & - & - & - & 4.55 & 4.29 \\
\hline & - & - & - & - & - & - & - & - & - & - & 12.70 & 12.3 \\
\hline & - & - & - & - & - & 20.15 & 20.27 & - & 18.79 & - & 17.49 & 19.43 \\
\hline & - & - & - & - & - & 2.87 & 3.41 & - & 2.98 & - & 2.50 & 2.84 \\
\hline & - & - & - & - & - & 5.21 & 5.06 & - & 5.30 & - & 5.30 & 4.97 \\
\hline $\mathrm{FI}$ & - & - & - & - & - & 14.05 & 12.94 & - & 12.85 & - & 12.06 & 12.59 \\
\hline & - & - & - & - & - & 17.79 & 18.54 & - & 17.36 & - & 14.29 & 14.27 \\
\hline & - & - & - & - & - & 6.31 & 6.65 & - & & - & 6.25 & 6.00 \\
\hline TI & - & - & - & - & - & 3.56 & 3.65 & - & 3.42 & - & 2.64 & 3.43 \\
\hline IDC & - & - & - & - & - & - & - & - & - & - & 10.01 & 10.11 \\
\hline BBC & - & - & - & - & - & - & - & - & - & - & 15.23 & 15.43 \\
\hline IDP & - & - & - & - & - & - & - & - & - & - & 11.21 & 11.37 \\
\hline & - & - & - & - & - & - & - & - & - & - & 5.21 & 4.34 \\
\hline$O C B$ & - & - & - & - & - & - & - & - & - & - & 7.98 & 8.03 \\
\hline
\end{tabular}

small and arranged in 14 transverse rows per centimeter at the base of the tail, in 15 rows per $\mathrm{cm}$ on the central portion of the tail, and in 21 rows on the apical centimeter of the tail $(n=1)$; tail brownish dorsally and ventrally.

Skull large and robust, proportionally long (Fig. 45; Table 35). Rostrum long and wide; rostral tube short (shorter than in Delomys, similar to Drymoreomys). Nasals long, not expanded laterally distally, with anterior margin projected beyond incisors and posterior margin (nasofrontal suture) extending beyond the maxillar-frontal-lacrimal sutures. Nasofrontal suture acute and triangular. Premaxillae short, with caudal margin anterior to the nasals. Lacrimal equally in contact with the maxillar and frontal. Zygomatic notch shallow. Interorbital region slightly divergent posteriorly, with sharp, moderately crested supraorbital margins, continuous with the moderately sharp temporal margin. Frontosquamosal suture not collinear with the frontoparietal suture. Zygomatic arches moderately robust, projected laterally, wider at the squamosal root. Braincase large and slightly elongat- ed. Parietals largely projected along the lateral surface of skull. Interparietal long and wide. Rostrum with gnathic process very short; nasals and premaxillar projected anteriorly forming a short rostral tube. Zygomatic plate narrow, with anterior margin straight; free dorsal margin barely developed. Zygomatic arches moderate, with jugal large with maxillar and squamosal processes not overlapping. Stapedial foramen large; sphenofrontal foramen and squamosal-alisphenoid groove present; large opening for the alisphenoid canal (primitive carotid and stapedial circulatory pattern 1; Voss, 1988). Alisphenoid strut absent, configuring oval accessory and buccinator-masticatory foramina confluent on a single foramen oval. Suspensory squamosal process present and overlapped to the tegmen tympani, configuring a very small postglenoid foramen. Subsquamosal fenestra very large. Stapedial process of ectotympanic present; external auditory meatus small and rounded. Mastoid perforated by small fenestra. Incisive foramina long, with lateral margins divergent posteriorly, wider slightly posteriorly; posterior margin reaching the anterior alveoli of $\mathrm{M} 1$. Posterior margin of zygomatic plate positioned anterior to the alveoli of M1. Palate wide and short, with two shallow lateral depression aligned with posterior palatine foramina, deeper medially; palate with posterolateral palatal pits simple on the palate level; palatal excrescencies absent. Mesopterygoid fossa penetrating between the molar series; roof of mesopterygoid fossa perforated by small sphenopalatine vacuities, positioned anteriorly to the presphenoid-basisphenoid suture. Parapterygoid fossa narrower than the mesopterygoid fossa. Auditory bullae moderately large and moderately globose, with the periotic margin extending to the internal carotid canal. Mandible long, with ramus high (Fig. 45). Condyloid process large and robust, projected posteriorly. Coronoid process moderately large, strongly falciform, leveled with the condyloid process; angular process moderately rounded, surpassing the condyloid process posteriorly; superior notch shallow and inferior notch moderately deep. Capsular process of lower incisor absent. Superior and inferior masseteric crests weakly developed, converging anteriorly and forming a single crest. Upper incisors opisthodont. Upper molar slightly divergent posteriorly; molar series long and wide, robust; molars with main cusps arranged in opposite pairs; main cusps moderately low, united by conspicuous enamel (or dentine) flexus; dentine exposition moderate; labial and lingual flexus conspicuous, moderately overlapping. M1 with procingulum divided by deep asymmetric anteromedian flexus; labial anteroconule noticeably larger than lingual one; anteroloph well developed; anteroflexus present; mesoloph robust, fused to mesostyle; mesoflexus short; protostyle present; enterostyle present; posteroloph present. $\mathrm{m} 1$ with procingulum developed, divided by a deep the anteromedian flexid; anterolophid present; mesolophid developed; protostylid and protolophid present; small; ectostylid present.

Remarks: $P$. ferrugineus was originally described in the genus Oryzomys (Thomas, 1894), but later Thomas (1917) moved the species to the new genus Phaenomys. The 


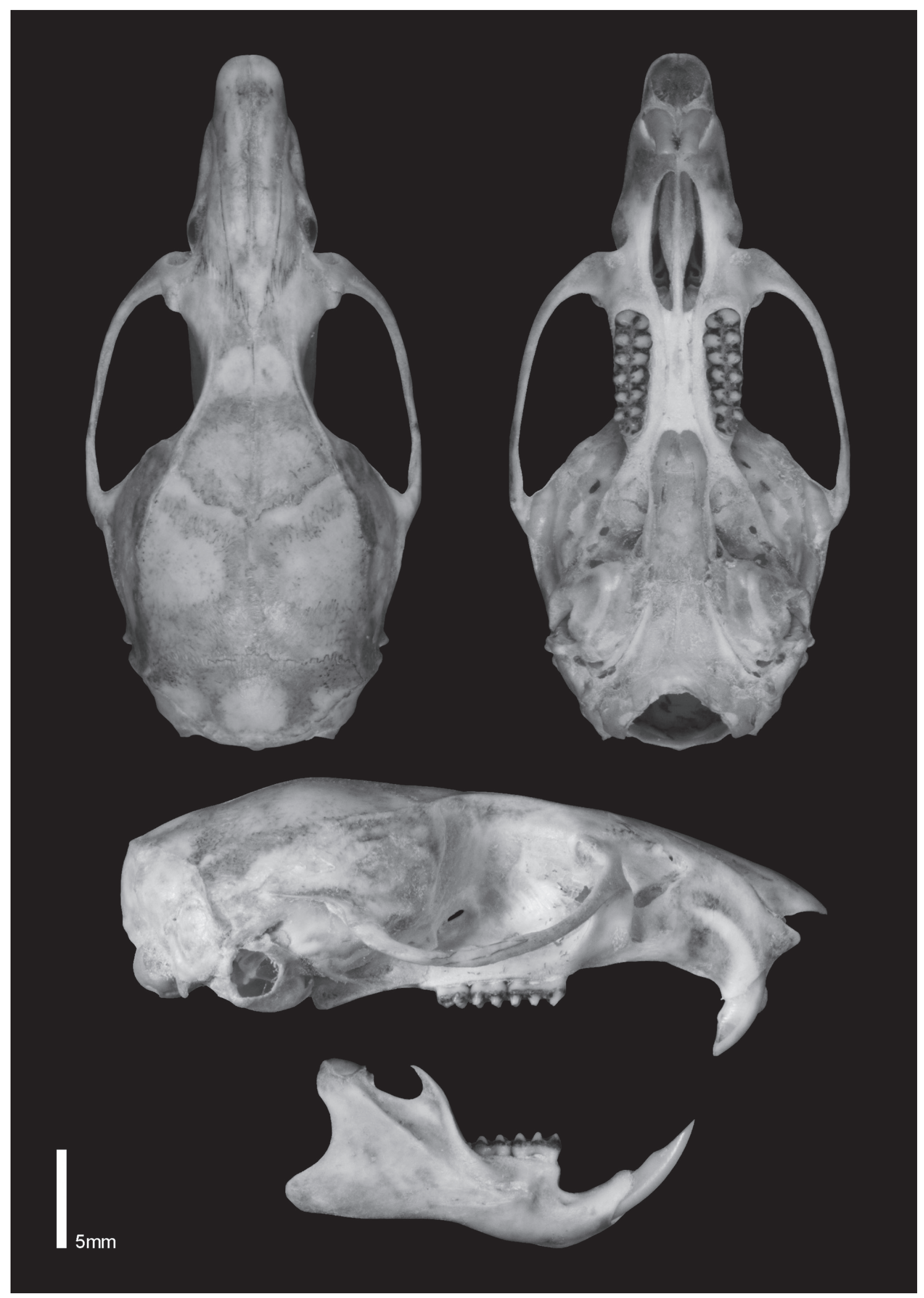

Figure 45. Skull and mandible of Phaenomys ferrugineus (EBM 190; male; GSL=35.96 mm). Skull on dorsal, ventral, and lateral view, and mandible on lateral view. 
description is based on the holotype, BMNH 76.12.8.3, which is a skin and skull, of undetermined sex from "Rio de Janeiro". Only 14 specimens are available in collections in Brazil and abroad, including the EEB specimen (Table 36). Some specimens were collected in the end of the XIX century, beginning of the XX century, and in the 1960 's (although not published until Vaz, 2000). For several years the species was not collected, but in 1998 a new specimen of $P$. ferrugineus, an adult male, was captured in Nova Friburgo, Rio de Janeiro, by the Laboratório da Peste da Fundação Nacional de Saúde (Bonvicino et al., 2001). More recently another specimen was obtained, an adult male captured in 2011 at Itamonte, Minas Gerais (Passamani et al., 2011). The EEB specimen was captured in 2004, and represents the second capture of $P$. ferrugineus from the Serra da Bocaina (the first was the specimen from the 1960's, from Fazenda California, Bananal, São Paulo; Percequillo, 2015b).

In Table 35 the measurement of the body and skull are presented for some specimens of $P$. ferrugineus. Some were extracted from the literature and some were measured directly from specimens. Bonvicino et al. (2001) made important observations on the external and cranial morphology of $P$. ferrugineus, which generally coincide with the EEB specimens.

Field observations: The only specimen of $P$. ferrugineus collected at EEB was a subadult male. It was captured in April, 2004, with pitfall trap, in area with arboreal vegetation with high and closed canopy.

Additional specimens examined: Phaenomys ferrugineus: Brazil: Minas Gerais: Casa Alpina Hotel, 14 km East of

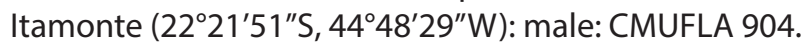

\section{Sigmodontinae incertae sedis Abrawayaomys ruschii Cunha \& Cruz 1979}

Voucher material: $\mathrm{n}=2$; male: MZUSP 34717; uncatalogued: EEB 816.

Specimens identification: Medium size (see body measurements on Table 37). Dorsal pelage moderately long, dense, and stiff, with spiny hairs, dark brown weakly grizzled with ochraceous yellow (grayish); guard hairs long (with about $11.5 \mathrm{~mm}$ in length), with half proximal portion thinner and grayish and distal portion wider and black; cover hairs moderately long (with nearly $10.5 \mathrm{~mm}$ ), with proximal five-sixths grayish and distal sixth portion ochraceous yellow; wool hairs very numerous, shorter, wavy and thin (with about $7.5 \mathrm{~mm}$ ), with the basal portion grayish and apical portion ( $1 / 6$ of the length of hairs) banded, with basal band ochraceous yellow and terminal band black. Lateral pelage similar to the dorsal pelage, predominantly grayish, mainly near the limit between lateral and ventral regions; transition with ventral region moderately subtle, not clearly delimited. Ventral pelage grayish, with hairs with basal half or basal third grayish and distal portion white or buffy. Mystacial vibrissae moderately long and dense, reaching the base of pinnae when laid backwards. Pinnae small and rounded, densely covered internally by brown hairs; pinnae moderately covered externally by short brown hairs. Manus short and moderately wide, covered dorsally by short grayish and whithish hairs; manus with moderately long digits bearing short claws (claw of dlll with about $2.2 \mathrm{~mm} ; \mathrm{n}=1$ ); dl vestigial, dll to dV longer, without conspicuous ungual tufts. Pes moderately long and wide, with dorsal surface covered by short grayish hairs; pes with dl shorter,

Table 36. Specimens of Phaenomys ferrugineus available in Brazilian collections (Museu Nacional da Universidade Federal do Rio de Janeiro, MNRJ; Museu de Zoologia da Universidade de São Paulo, MZUSP; Coleção de Mamíferos da Universidade Federal de Lavras, CMUFLA) and abroad (The Natural History Museum, London, BMNH; Museum für Naturkunde, der Humboldt Universität zu Berlin, Berlin, ZMB). Information obtained from Vaz (2000), Bonvicino et al. (2001), Passamani et al. (2011) and present study. ${ }^{*}$ Skull of specimen BMNH 48.5.6.8, according to Vaz (2000). ${ }^{* *}$ Coordinates provided by Passamani et al. (2011).

\begin{tabular}{|c|c|c|c|c|c|c|c|}
\hline Specimen & Collector & Locality & Coordinates & Altitude & Date & Sex & Material \\
\hline BMNH 76.12.8.3 (Holotype) & - & "Rio de Janeiro" & - & - & - & - & Skin and skull \\
\hline BMNH 63.11.16.2 & - & "Bahia" & - & - & - & female & Skin and skull \\
\hline BMNH 27.11.19.43 & - & "Brésil" & - & - & - & female & Skin and skull \\
\hline BMNH 48.5.6.8 & - & "Brazil" & - & - & - & - & Skin \\
\hline BMNH 48.5.28.1* & - & "Brasil" & - & - & - & - & Skull \\
\hline ZMB 3705 & - & "Brazilien" & - & - & - & male & Skin \\
\hline ZMB 3706 & - & "Brazilien" & - & - & - & female & Skin \\
\hline ZMB 26999 & E.L. Bresslau and P.F. Schirch & "Therezopolis, Socavão" & $22^{\circ} 22^{\prime} S, 42^{\circ} 44^{\prime} \mathrm{W}^{* *}$ & & 16.IV.1914 & - & Skin and skull \\
\hline MNRJ 1992 & - & Teresópolis & - & - & - & - & Skin and skull \\
\hline MNRJ 2124 & P.F. Schirch & Teresópolis & - & - & 25.IX.1914 & male & Skin and skull \\
\hline MNRJ 2125 & P.F. Schirch & Teresópolis & - & - & 1915 & female & Skin \\
\hline MNRJ 53614 & - & $\begin{array}{l}\text { Córrego Grande, } 26 \text { km NE of "Socavão", } \\
\text { Nova Friburgo, Rio de Janeiro State, Brazil }\end{array}$ & $22^{\circ} 17^{\prime} 12^{\prime \prime} \mathrm{S}, 42^{\circ} 41^{\prime} 28^{\prime \prime} \mathrm{W}$ & $1,080 \mathrm{~m}$ & 09.IX.1998 & male & $\begin{array}{l}\text { Skin, skull, and partial } \\
\text { postcranial skeleton }\end{array}$ \\
\hline MZUSP 6004 & A.M. Olalla & $\begin{array}{l}\text { "Serra da Bocaina". According Vaz (2000), } \\
\text { nearby Fazenda Califórnia, Bananal } \\
\text { municipality, São Paulo State, Brazil }\end{array}$ & $22^{\circ} 53^{\prime} 00^{\prime \prime} \mathrm{S}, 44^{\circ} 28^{\prime} 30^{\prime \prime} \mathrm{W}$ & & 28.VIII. 1941 & male & Skin \\
\hline $\begin{array}{l}\text { MZUSP not cataloged } \\
\text { (EBM 190) }\end{array}$ & A.R. Percequillo and C.C. Aires & $\begin{array}{l}\text { Estação Ecológica de Bananal, Bananal } \\
\text { municipality, São Paulo State, Brazil }\end{array}$ & $22^{\circ} 47^{\prime} 42^{\prime \prime} \mathrm{S}, 44^{\circ} 21^{\prime} 36^{\prime \prime} \mathrm{W}$ & $1,200 \mathrm{~m}$ & 19.IV.2004 & male & $\begin{array}{l}\text { Skin, skull, postcranial skeleton, } \\
\text { tissue, and ectoparasites }\end{array}$ \\
\hline CMUFLA 904 & M. Passamani & $\begin{array}{l}\text { Casa Alpina Hotel, } 14 \text { km E of Itamonte, } \\
\text { Minas Gerais State, Brazil }\end{array}$ & $22^{\circ} 21^{\prime} 51^{\prime \prime S}, 44^{\circ} 48^{\prime} 29^{\prime \prime} \mathrm{W}$ & $1,550 \mathrm{~m}$ & 12.II.2011 & male & Skin, skull, and tissue \\
\hline
\end{tabular}


Table 37. Body and cranio-dental morphometric values (mm), and weight (g) of Abrawayaomys. *Data from Pardiñas et al. (2009).

\begin{tabular}{|c|c|c|c|c|c|c|c|c|c|c|}
\hline & CEM 9970* & MACN 20253* & MN 23075* & MN 67557* & MN* & UFMG 2492* & UFSC $3427^{*}$ & USNM 552416 & MZUSP 34717 & EEB 816 \\
\hline Sex & - & male & female & male & male & male & male & male & male & male \\
\hline Age Class & - & 4 & 3 & 3 & - & 4 & - & 3 & 2 & 1 \\
\hline HBL & - & 120.0 & 116.0 & 135.0 & 122.0 & 128.0 & 116.0 & 140.0 & 100.0 & 75.0 \\
\hline TL & - & 133.0 & 85.0 & [116] & 144.0 & 146.0 & 124.0 & 150.0 & [103] & 90.0 \\
\hline HF1 & - & 27.5 & - & - & - & - & - & - & 27.0 & 24.0 \\
\hline HF2 & - & 29.0 & 29.0 & 32.0 & 30.0 & 31.0 & 32.0 & 30.0 & 29.0 & 26.0 \\
\hline EL & - & 17.0 & 20.0 & 16.0 & 20.0 & 20.0 & 18.0 & - & 16.0 & 15.0 \\
\hline W & - & - & 46.0 & 55.0 & 59.0 & 63.0 & 42.0 & - & 27.5 & - \\
\hline GSL & - & 27.67 & 27.46 & 28.64 & - & 27.64 & - & 26.98 & 26.04 & 23.59 \\
\hline CIL & - & - & - & - & - & - & - & 26.45 & 24.68 & 22.43 \\
\hline CZL & - & - & - & - & - & - & - & 21.33 & 19.74 & 17.62 \\
\hline LD & - & 7.68 & 7.57 & 8.43 & - & 7.97 & - & 7.04 & 6.56 & 6.03 \\
\hline LIF & 4.40 & 4.65 & 5.33 & 5.56 & - & 4.83 & - & 4.92 & 4.29 & 4.20 \\
\hline BIF & 1.20 & 1.44 & 1.72 & 1.63 & - & 1.68 & - & 1.57 & 1.70 & 1.76 \\
\hline LM & 3.30 & 3.96 & 4.51 & 4.25 & - & 4.62 & - & 4.38 & 4.60 & - \\
\hline BM1 & - & 1.16 & 1.32 & 1.29 & - & 1.39 & - & 1.32 & 1.37 & 1.38 \\
\hline ID & - & - & - & - & - & - & - & 1.97 & 1.80 & 1.34 \\
\hline IW & - & - & - & - & - & - & - & 1.76 & 1.68 & 1.26 \\
\hline LN & - & - & - & - & - & - & - & 9.06 & 8.31 & 7.01 \\
\hline RL & - & 10.94 & 8.26 & 8.41 & - & 9.15 & - & 7.87 & 7.99 & 6.81 \\
\hline ZB & 16.00 & 16.99 & 17.20 & 17.40 & - & 16.00 & - & 16.44 & 15.41 & 14.02 \\
\hline BZP & - & 3.56 & 4.34 & 4.31 & - & 3.70 & - & 3.10 & 2.74 & 2.51 \\
\hline LIB & 5.90 & 6.02 & 6.29 & 6.21 & - & 6.23 & - & 5.92 & 6.22 & 5.91 \\
\hline OFL & - & 9.81 & 10.10 & 11.06 & - & 10.70 & - & 10.10 & 9.00 & 8.46 \\
\hline PL & - & - & - & - & - & - & - & 11.75 & 10.73 & 9.72 \\
\hline LPB & - & 5.25 & 5.46 & 5.21 & - & 5.87 & - & 5.06 & 4.76 & 4.44 \\
\hline HBC & 11.0 & 9.68 & 9.94 & 10.34 & - & 10.22 & - & 9.70 & 9.53 & 9.17 \\
\hline BBC & - & 13.67 & 13.18 & 13.57 & - & 13.08 & - & 13.13 & 13.43 & 12.84 \\
\hline IPB & - & - & - & - & - & - & - & 4.77 & 3.29 & 3.87 \\
\hline IPL & - & - & - & - & - & - & - & 3.00 & 2.95 & 1.42 \\
\hline$O C B$ & - & 6.53 & 8.01 & 8.00 & - & 7.07 & - & 7.04 & 7.35 & 6.83 \\
\hline
\end{tabular}

dIII longer than dII and dIV; digits with moderately long claws (claw of dIII with about $2.7 \mathrm{~mm} ; \mathrm{n}=1$ ); digits with sparse and long ungual tufts, not completely concealing the claws; ungual tufts with gray base and white apex; pes with moderately large interdigital pads; thenar and hypothenar pads present; plantar integument between pads covered by squamae; pads flesh colored. Tail longer than head and body length; tail covered by short hairs, whose length equals the length of one to two scale rows on dorsal and ventral surface; apical tuft of hairs absent; scales small and arranged in 20 transverse rows per centimeter at the base and central portion of the tail, and in 24 rows on the apical centimeter of the tail $(n=1)$; tail brownish dorsally (hairs entirely brown), brownish ventrally (hairs with brown base and white apex, more concentrated on the base of the tail).

Skull small, short and wide, robustly built (Fig. 46; Table 37). Rostrum very short and narrow; rostral tube absent. Nasals short, not expanded laterally distally, with anterior margin not projected anteriorly beyond incisors and posterior margin (nasofrontal suture) surpassing or not the maxillar-frontal-lacrimal sutures. Nasofrontal suture triangular and acute or squared. Premaxillae long, leveled to the nasals. Lacrimal equally in contact with the maxillar and frontal. Zygomatic notch shallow to moderately deep and narrow. Interorbital region slightly diver- gent posteriorly, narrower anteriorly, with supraorbital margins rounded, or symmetrically constricted (hourglass), with squared supraorbital margins; supraorbital margins continuous with the moderately sharp temporal margin. Frontosquamosal suture not collinear with the frontoparietal suture. Zygomatic arches delicate, projected laterally, wider at the squamosal root. Braincase large and voluminous, with long profile. Parietals largely projected along the lateral surface of skull. Interparietal very small, narrow and short, diamond-shaped. Rostrum with gnathic process short. Zygomatic plate moderately wide, with anterior margin straight or very slightly concave; free dorsal margin projected anteriorly, with rounded and downward sloping anterodorsal margin. Zygomatic arches moderately built, with jugal large with maxillar and squamosal processes not overlapping. Stapedial foramen large, sphenofrontal foramen present and squamosal-alisphenoid groove present; large opening for the alisphenoid canal (primitive carotid and stapedial circulatory pattern 1; Voss, 1988). Alisphenoid strut absent, configuring oval accessory and buccinator-masticatory foramina confluent, on a single foramen oval, or present, with distinct oval accessory and buccinator-masticatory foramina. Suspensory squamosal process present and overlapped to the tegmen tympani, configuring a small postglenoid foramen. Subsquamosal fenestra very large. 


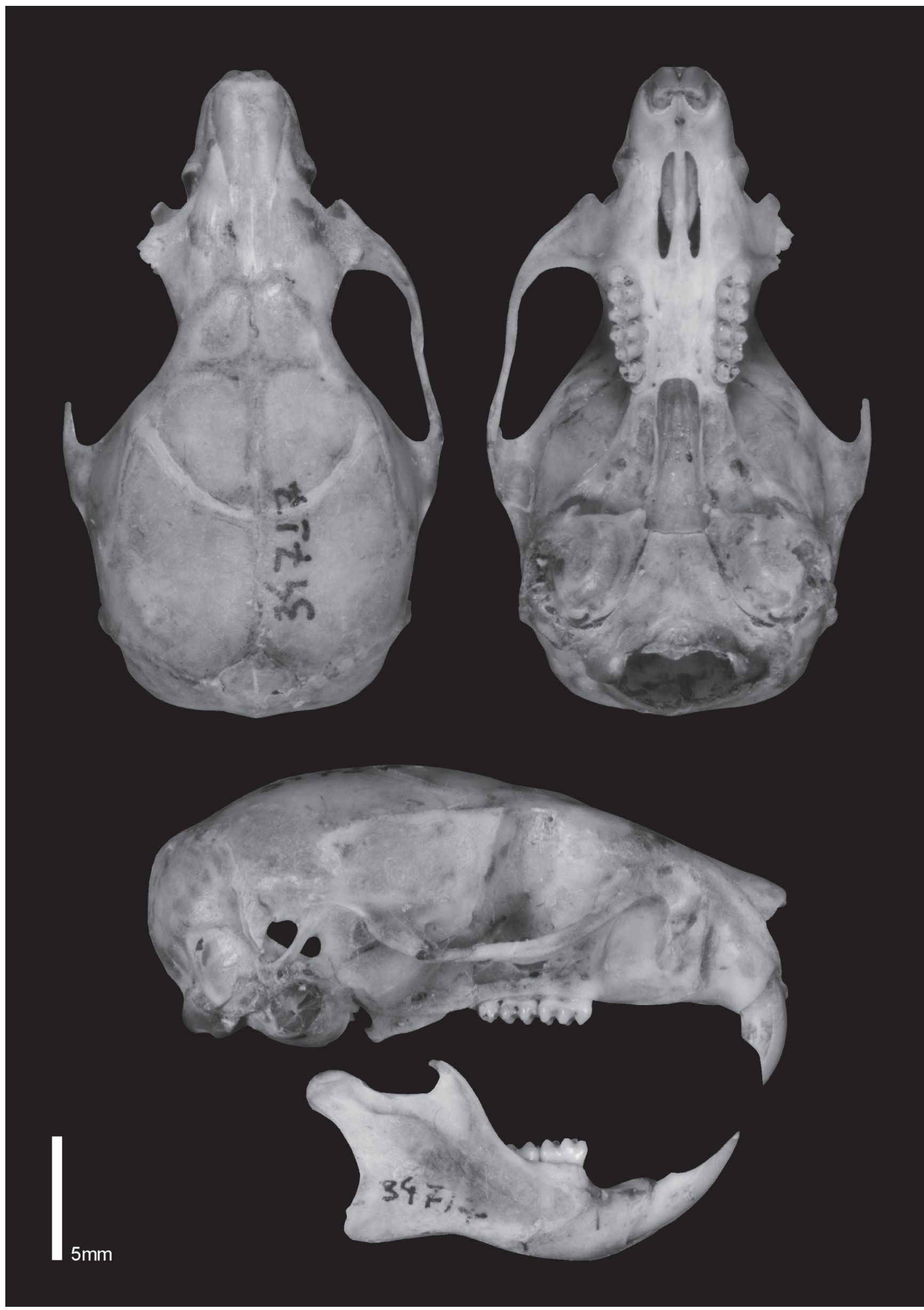

Figure 46. Skull and mandible of Abrawayaomys ruschii (MZUSP 34717; male; GSL =26.04 mm). Skull on dorsal, ventral, and lateral view, and mandible on lateral view. 
Stapedial process of ectotympanic present; external auditory meatus small and rounded; mastoid perforated by small fenestra. Incisive foramina moderately long and narrow, with lateral margins parallel; posterior margin not reaching the anterior alveoli of M1. Posterior margin of zygomatic plate positioned nearly to the alveoli of M1. Palate wide, long and flat, with deep central depression or with two shallow lateral depression aligned with posterior palatine foramina; palate with posterolateral palatal pits simple, not positioned in depressions, or absent; palatal excrescencies absent. Mesopterygoid fossa penetrating or not between molar series; roof of mestopterygoid fossa not perforated by sphenopalatine vacuities. Parapterygoid fossa narrower than the mesopterygoid fossa. Auditory bullae small and moderately globose, with the periotic margin extending to the internal carotid canal. Mandible elongated, with ramus high (Fig. 46). Condyloid process robust and long. Coronoid process developed, falciform, on the same level of the condyloid process; angular process rounded, positioned much anteriorly than condyloid process; superior notch moderately deep and inferior notch asymmetric and moderately deep. Capsular process of lower incisor strongly developed. Superior and inferior masseteric crests weakly developed, converging in a "V" shape anteriorly. Upper incisors proodont. Upper molar slightly convergent posteriorly; molar series short and narrow; molars with main cusps arranged in slightly alternate pairs, with lingual cusps anteriorly positioned than labial ones; main cusps moderately high, united by conspicuous enamel (or dentine) flexus; dentine exposition small; labial and lingual flexus incipient, not overlapping. $\mathrm{M} 1$ with procingulum divided or not by an asymmetric anteromedian flexus; labial anteroconule slightly larger than lingual one; anteroloph apparently fused to the conule; anterior mure present, as a small but distinct cone; mesoloph fused to the paracone, with a labial portion projected posteriorly and fused to the metacone; paracone not connected to the median mure or to the protocobe, forming a very long paraflexus; mesoflexus very short; protoloph present or not; enterostyle absent; posteroloph present, but small. $\mathrm{m} 1$ with procingulum very small, not divided by the anteromedian flexid; anterolophid absent; mesolophid absent; protostylid and ectostylid small; ectostylid and ectolophid present.

Remarks: The genus Abrawayaomys was recently reviewed by Percequillo et al. (2017). These authors, showed that most of the morphologic features used to distinguish the two previously valid species are highly polymorphic and also reported a low genetic divergence between them, suggesting that $A$. chebezi Pardiñas, Teta, and D'Elía, 2009 is a junior synonym of $A$. ruschii Cunha and Cruz, 1979. The specimens collected at EEB were included in the analyses of Percequillo et al. (2017).

Field observations: The two EEB specimens were captured with pitfall traps; the first capture was in December 2003 (subadult male) and the second specimen was trapped in April 2004 (juvenile male).

\section{Genus Delomys}

The most recent taxonomic review of Delomys (Gonçalves \& Oliveira, 2014) suggests that the genus includes three species. Two of them, D. dorsalis and D. sublineatus, were identified in the EEB samples, based on external and cranio-dental traits. On the external morphology, the dorsal pelage soft and dense of $D$. dorsalis differs from the dorsal pelage coarse and sparser of $D$. sublineatus; the dorsal coloration dark brownish of $D$. dorsalis differs from the orangish dorsal coloration of $D$. sublineatus, which also presents an orangish strip between the lateral and ventral pelage; $D$. dorsalis has three mammae pairs, while $D$. sublineatus has four; the tail of $D$. sublineatus is clearly more bicolor than the tail of $D$. dorsalis; the hindfeet are covered by whitish hairs in $D$. sublineatus and by grayish/brownish hairs in $D$. dorsalis; mystacial vibrissae are longer in $D$. dorsalis (always reaching the base of pinnae when laid back) and shorter in D. sublineatus (mostly not reaching the base of pinnae when laid back); and the ventral color is strongly grayish in $D$. dorsalis, while in $D$. sublineatus it is whitish.

No cranial qualitative characteristic was found to distinguish between $D$. dorsalis and $D$. sublineatus, as previously suggested by Voss (1993) and Gonçalves \& Oliveira (2014), even though there is a slight divergence in the frequencies of some characters states. However, it is worth to mention, that in the M1 of $D$. sublineatus the anterconules are asymmetrical - the anteromedian flexus is positioned more lingually, defining a lingual anteroconule that is smaller than the labial anteroconule; in $D$. dorsalis, by contrast, the anteroconules are symmetrical - with anteromedian flexus positioned medially to the procingulum. Furthermore, analysis of an extensive series of both taxa allowed us to notice that the cranium of $D$. dorsalis is slightly more robust and wider, whereas the cranium of $D$. sublineatus is narrower and slender, which was confirmed by the influence of the width of the interorbital in the multivariate analysis (see below).

These two species are also well-defined through cranio-dental morphometric characteristics. A PCA with 22 variables (see variables list in Table 38 ) was performed and a clear segregation was found. The PCA revealed that the populations of $D$. dorsalis and $D$. sublineatus from EEB can be distinguished without overlap of individual scores along the second principal component (Fig. 47); this represented $3.5 \%$ of the variation and was determined by: interparietal breadth (IPB), least interorbital breadth (LIB), height of braincase (HBC) length of molars (LM) (Table 36). The first principal component corresponded to $91.4 \%$ of the variation, and the variables related to it, as the cranium length (GSL and CIL) and rostrum length ( $L D$ and $R L$ ), are responsible for most of the variation on this component.

Voss (1993) documented the multivariate craniometric divergence in samples of $D$. dorsalis and $D$. sublineatus from Estação Biológica de Boracéia, SP. The author applied a growth correction to level off the specimens in different age groups, which may not be necessary for discrimination, based on the results of the present anal- 
Table 38. Scores of cranio-dental morphometric variables in the first two principal components from a PCA of Delomys. Variables that loaded higher on each component are indicated in bold.

\begin{tabular}{lcc}
\hline Variables & $\mathbf{P C 1}$ & $\mathbf{P C 2}$ \\
\hline GSL & $\mathbf{0 . 9 9 7}$ & 0.032 \\
CIL & $\mathbf{0 . 9 9 5}$ & -0.051 \\
CZL & 0.960 & -0.218 \\
LD & $\mathbf{0 . 9 7 2}$ & 0.088 \\
LIF & 0.921 & 0.088 \\
BIF & 0.706 & 0.095 \\
LM & 0.320 & $\mathbf{0 . 4 1 5}$ \\
BM1 & 0.633 & 0.402 \\
IW & 0.858 & -0.209 \\
LN & 0.960 & -0.129 \\
BR & 0.611 & 0.131 \\
RL & $\mathbf{0 . 9 7 1}$ & 0.140 \\
BZP & 0.864 & -0.078 \\
LIB & 0.418 & $\mathbf{0 . 7 3 1}$ \\
OFL & 0.890 & -0.315 \\
PL & 0.966 & 0.112 \\
LPB & 0.600 & -0.075 \\
BBP & 0.816 & 0.094 \\
HBC & 0.762 & $\mathbf{0 . 4 1 9}$ \\
BBC & 0.752 & 0.409 \\
IPB & 0.453 & $\mathbf{0 . 7 9 1}$ \\
IPL & 0.547 & 0.301 \\
\hline
\end{tabular}

ysis, which included specimens in age-groups AC1 to AC4. A possible explanation for the agreement between our results and those of Voss (1993) despite the different treatment of samples, is the fact that the second component, which was responsible for the separation between the species, is related to the shape, and not size, of organisms. Shape is determined by ontogenetic factors whereas the first component is more correlated with size (Hingst, 1995).

This cranial morphometric segregation revealed the importance of one variable that can be used to distinguish $D$. dorsalis and $D$. sublineatus, interorbital width (LIB). This measurement strongly influenced the dispersion of individual scores along the second component, both in the present study and in the analysis of Voss (1993). These results confirm that the interorbital of $D$. dorsalis is wider (between 5.16 and $5.89 \mathrm{~mm}$ ) in EEB specimens, compared with $D$. sublineatus (between 4.74 and $5.14 \mathrm{~mm}$ ); the values of this variable did not overlap, and they did not vary significantly with ontogenetic development in any of the species in question, suggesting that this is a reliable variable for species diagnosis.

Furthermore, we tested the presence of discontinuities between these species in body measurements, through a bivariate graph of HBL versus TL. The body variables were also completely distinct in Delomys species. Specimens representing $D$. dorsalis occupied the upper diagonal of the graph and the specimens of $D$. sublineatus the lower diagonal (Fig. 48). In the individuals of $D$. dorsalis analyzed, the tail corresponds, on average, to $103.5 \%( \pm 7.1 ; n=41)$ of the length of head and body, varying from 88.2 to $116.3 \%$; in D. sublineatus, on the other hand, the tail corresponds to $84.1 \%( \pm 7.4 ; n=18)$ of

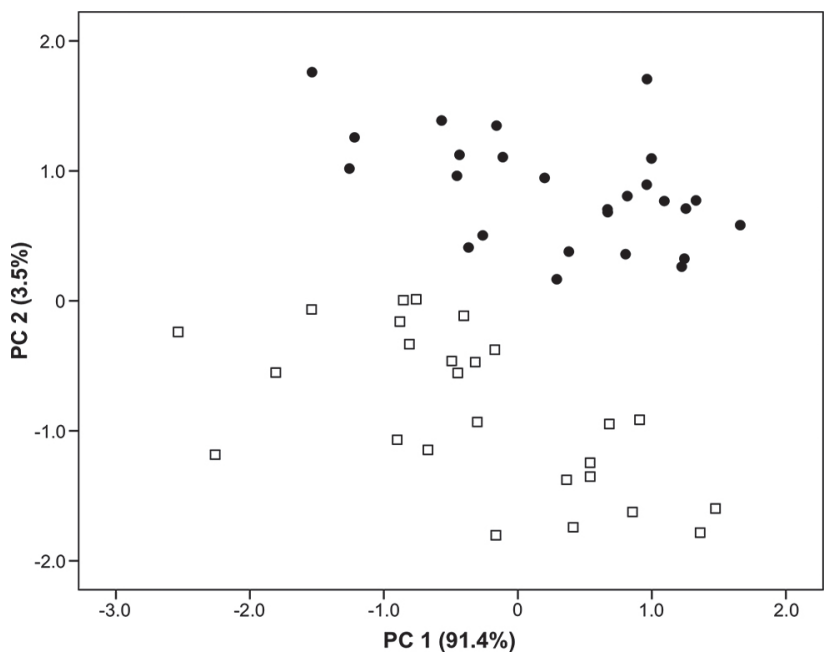

Figure 47. Scatterplot graph showing the projections of individual scores of Delomys dorsalis (clack dots) and Delomys sublineatus (white squares) specimens, from a PCA analysis.

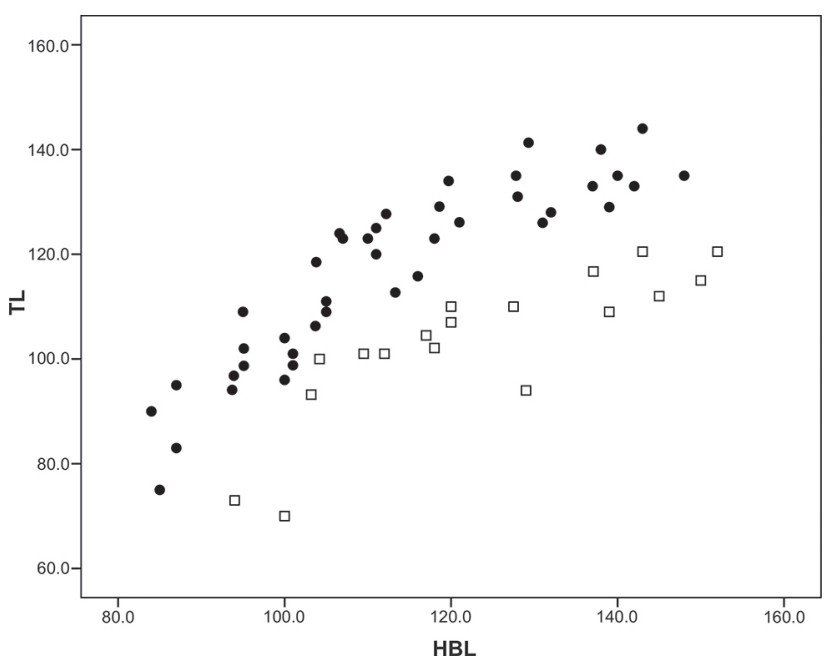

Figure 48. Biplot graph displaying the head and body length (HBL) and the tail length (TL) of Delomys dorsalis (black dots) and Delomys sublineatus (white squares) specimens.

the length of head and body, between 70.0 and $96.0 \%$. Despite the partial overlap in values, we can state that the tail of $D$. dorsalis is similar in size or longer than the combined length of head and body and the tail of $D$. sublineatus is always smaller than the combined length of head and body. In conclusion, as observed by Voss (1993) in the population from Boracéia, the relationship $\mathrm{HBL}$ versus $\mathrm{TL}$ is an important diagnostic characteristic among species of the genus Delomys, corroborated here for EEB specimens.

\section{Delomys dorsalis (Hensel 1872)}

Voucher material: $\mathrm{n}=52$; female: MZUSP 33677, 33678, 33719, 33724, 33777, 33778, 33780; uncatalogued: EBM 017, 044, 059, 061, 099, 249, 261; EEB 554, 569, 657, 737, 739, 770, 929; male: MZUSP 33722, 33728, 33768, 33774, 33776, 33793; uncatalogued: EBM 052, 093, 110, 150, $151,158,168,194,218,220,243,262$, 337; EEB 535, 544, 
Table 39. Descriptive statistics (mean \pm standard deviation, range, and sample size) of body and cranio-dental measurements $(\mathrm{mm})$, and weight $(\mathrm{g})$, of adult specimens of Delomys dorsalis and Delomys sublineatus from EEB.

\begin{tabular}{|c|c|c|}
\hline & $\begin{array}{l}\text { Delomys dorsalis } \\
\text { (6 males and } 9 \text { females) }\end{array}$ & $\begin{array}{l}\text { Delomys sublineatus } \\
\text { (7 males and } 3 \text { females) }\end{array}$ \\
\hline HBL & $133.04 \pm 10.21(106.6-148.0) 15$ & $137.57 \pm 12.19(117.0-152.0) 9$ \\
\hline TL & $133.33 \pm 5.93(124.0-144.0) 13$ & $113.24 \pm 5.76(104.5-120.5) 8$ \\
\hline HF1 & $28.59 \pm 1.15(25.9-30.5) 15$ & $28.28 \pm 1.06(27.0-30.0) 10$ \\
\hline HF2 & $30.07 \pm 1.26(26.6-32.0) 15$ & $30.28 \pm 0.94(29.0-32.0) 9$ \\
\hline EL & $21.23 \pm 2.20(17.0-25.0) 13$ & $21.87 \pm 1.17(20.7-24.0) 10$ \\
\hline W & $75.70 \pm 9.89(64.0-95.0) 10$ & $68.81 \pm 12.62(41.0-79.0) 8$ \\
\hline GSL & $33.97 \pm 0.95(32.40-35.97) 15$ & $33.11 \pm 1.45(30.34-35.08) 9$ \\
\hline CIL & $29.63 \pm 0.70(28.04-30.63) 14$ & $29.28 \pm 1.17(27.23-31.16) 9$ \\
\hline CZL & $21.77 \pm 0.64(20.63-23.14) 14$ & $22.13 \pm 0.87(20.85-23.46) 9$ \\
\hline LD & $9.20 \pm 0.33(8.52-9.76) 15$ & $8.64 \pm 0.60(7.33-9.34) 10$ \\
\hline LIF & $7.17 \pm 0.33(6.69-7.83) 15$ & $6.73 \pm 0.45(5.88-7.27) 10$ \\
\hline BIF & $2.66 \pm 0.19(2.40-3.12) 15$ & $2.47 \pm 0.19(2.06-2.65) 9$ \\
\hline LM & $4.81 \pm 0.13(4.47-5.01) 15$ & $4.72 \pm 0.18(4.39-4.92) 10$ \\
\hline BM1 & $1.51 \pm 0.07(1.40-1.65) 15$ & $1.43 \pm 0.06(1.34-1.54) 10$ \\
\hline ID & $1.46 \pm 0.07(1.35-1.58) 15$ & $1.44 \pm 0.10(1.29-1.61) 10$ \\
\hline IW & $1.74 \pm 0.10(1.60-1.92) 14$ & $1.65 \pm 0.09(1.49-1.80) 10$ \\
\hline LN & $13.65 \pm 0.65(12.34-14.92) 15$ & $13.55 \pm 1.03(12.18-15.12) 9$ \\
\hline BR & $4.71 \pm 0.28(4.28-5.46) 15$ & $4.52 \pm 0.26(4.29-5.12) 10$ \\
\hline $\mathbf{R L}$ & $14.12 \pm 0.57(13.29-15.27) 15$ & $13.27 \pm 0.82(11.50-14.46) 9$ \\
\hline ZB & $16.21 \pm 0.65(15.09-17.07) 13$ & $15.99 \pm 0.73(14.69-16.85) 7$ \\
\hline BZP & $2.97 \pm 0.19(2.54-3.20) 15$ & $2.88 \pm 0.19(2.67-3.24) 10$ \\
\hline LIB & $5.42 \pm 0.21(5.16-5.89) 15$ & $4.94 \pm 0.13(4.74-5.14) 10$ \\
\hline OFL & $10.08 \pm 0.34(9.33-10.71) 15$ & $10.32 \pm 0.39(9.74-10.84) 9$ \\
\hline PL & $13.41 \pm 0.43(12.71-14.07) 15$ & $12.77 \pm 0.61(11.55-13.46) 10$ \\
\hline LPB & $4.91 \pm 0.29(4.54-5.57) 15$ & $4.87 \pm 0.34(4.36-5.62) 10$ \\
\hline BBP & $3.32 \pm 0.21(2.99-3.65) 15$ & $3.13 \pm 0.21(2.79-3.42) 10$ \\
\hline HBC & $9.38 \pm 0.38(8.61-10.03) 15$ & $8.98 \pm 0.36(8.58-9.61) 9$ \\
\hline BBC & $14.06 \pm 0.44(13.50-14.91) 15$ & $13.37 \pm 0.36(12.90-14.03) 9$ \\
\hline IPB & $10.97 \pm 0.47(10.14-11.67) 15$ & $9.75 \pm 0.52(8.78-10.34) 9$ \\
\hline IPL & $3.92 \pm 0.33(3.50-4.50) 15$ & $3.67 \pm 0.39(3.08-4.19) 9$ \\
\hline$O C B$ & $7.34 \pm 0.28(6.75-7.87) 14$ & $7.05 \pm 0.26(6.69-7.55) 10$ \\
\hline
\end{tabular}

561, 700, 740, 741, 743, 759; sex unknown: MZUSP 33723; uncatalogued: EBM 301; EEB 692, 866.

Specimens identification: Large size (see body measurements on Table 39). Dorsal pelage moderately long and dense, dark brown moderately speckled with ochraceous yellow, with a distinct longitudinal darker stripe from the nape to the base of the tail; guard hairs long (length ranging from 11.5 to $12 \mathrm{~mm}$ ), with half proximal portion thinner and grayish and distal portion wider and with a basal band dark gray and apical band ochraceous brown; cover hairs moderately long (length ranging from 7.5 to10 $\mathrm{mm}$ ), with proximal two thirds grayish and distal portion with a basal band dark gray and apical band ochraceous brown; wool hairs numerous, shorter, wavy and thin (length ranging from 6 to $9.5 \mathrm{~mm}$ ), grayish colored with a subterminal band brown and a terminal band black. Lateral pelage similar to the dorsal pelage, dark brown more intensely grizzled with ochraceous yellow; transition with ventral region well defined, clearly delimited. Ventral pelage grayish, with hairs with basal half or basal third grayish and distal portion white. Three mammae pairs, in inguinal, abdominal and postaxial positions
( $n=8$ ). Mystacial vibrissae moderately long, reaching the base of pinnae when laid backwards. Pinnae small and rounded, weakly covered internally and externally by brown hairs. Manus short and moderately narrow, covered dorsally by short hairs, with basal portion brown and apical white; manus with moderately long digits bearing very short claws (claw of dlll with length ranging from 0.8 to $1.3 \mathrm{~mm} ; \mathrm{n}=5$ ); dl vestigial, dll to $\mathrm{dV}$ longer, without conspicuous ungual tufts. Pes long and narrow, with dorsal surface covered by short and whitish hairs; pes with dl shorter, dIII equal or longer than dII and equal or shorter than dIV; digits with moderately long claws (claw of dIII ranging from 2.5 to $3 \mathrm{~mm} ; \mathrm{n}=5$ ); digits with sparse and moderately long ungual tufts, not completely concealing the claws; ungual tufts whitish, as well as digits; pes with moderately large interdigital pads; thenar and hypothenar pads present; plantar integument between pads covered by squamae; pads flesh colored. Tail similar to head and body length $(103.5 \% \pm 7.1$ of head and body length, ranging from 88.2 to $116.3 \% ; n=41$ ); tail covered by short hairs, whose length equals the length of one to two scale rows on dorsal and ventral surface; apical tuft of hairs absent; scales small and arranged in 14 to 18 transverse rows per centimeter at the base of the tail, between 13 to 16 rows per $\mathrm{cm}$ on the central portion of the tail, and between 18 to 22 rows on the apical centimeter of the tail $(n=5)$; tail brownish dorsally (hairs entirely brown), brownish ventrally (hairs with brown base and white apex, more concentrated on the base of the tail).

Skull long and narrow (Fig. 49; Table 39). Rostrum very long and narrow; rostral tube present and long; anterior portion of premaxillae and nasals projected anteriorly. Nasals long, not expanded laterally distally, with anterior margin projected beyond incisors and posterior margin (nasofrontal suture) surpassing (3.1\%) or not (96.9\%) the maxillar-frontal-lacrimal sutures $(n=32)$. Nasofrontal suture rounded. Premaxillae short, with caudal margin anterior $(31.3 \%)$ to or leveled $(65.6 \%)$ to the nasals, or long, extending beyond the nasals $(3.1 \%)(n=32)$. Lacrimal equally in contact with the maxillar and frontal (80\%) or predominantly in contact with maxillar $(20 \%)(n=32)$. Zygomatic notch moderately deep to deep, and narrow. Interorbital region symmetrically constricted (hourglass), with rounded (75\%) or sharp, squared (25\%; especially more posteriorly) supraorbital margins ( $n=32)$; supraorbital margins continuous with the moderately sharp temporal margin. Frontosquamosal suture collinear $(31.25 \%)$ or not $(68.75 \%)$ with the frontoparietal suture $(n=32)$. Zygomatic arches delicate, projected laterally, wider at the squamosal root. Braincase small and rounded. Parietals mostly restricted to the dorsal surface of skull. Interparietal long and wide. Rostrum with gnathic process very short. Zygomatic plate moderately wide, with anterior margin straight or very slightly concave; free dorsal margin projected anteriorly, with rounded and downward sloping anterodorsal margin (61.3\%) or projected anteriorly with distinct anterior process (38.7\%) ( $n=32)$. Zygomatic arches moderate, with jugal large with maxillar and squamosal processes not overlapping (96.8\%) or jugal small, with process overlapped, 


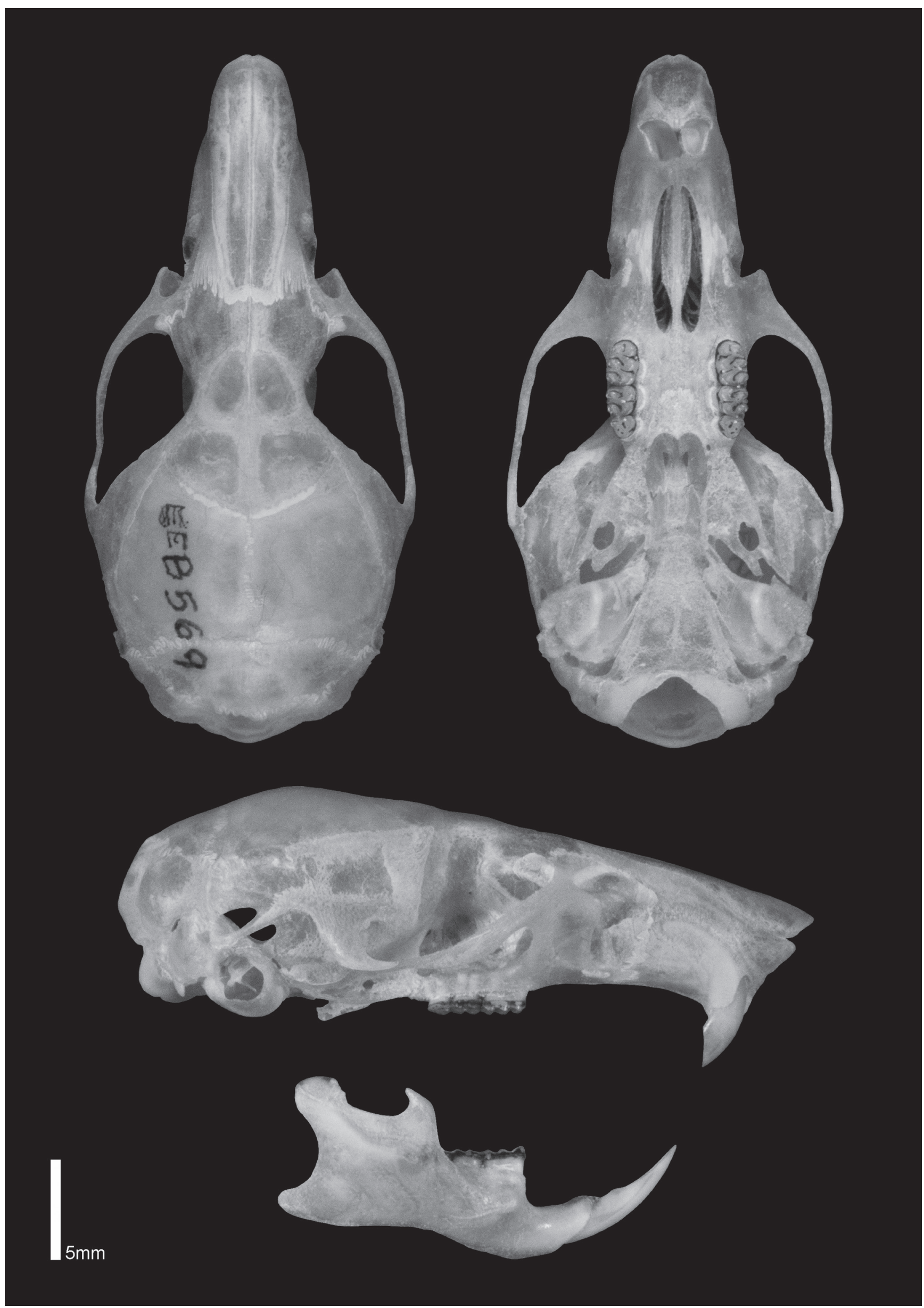

Figure 49. Skull and mandible of Delomys dorsalis (EEB 569; female; GSL = $34.69 \mathrm{~mm}$ ). Skull on dorsal, ventral, and lateral view, and mandible on lateral view. 
but not in contact $(3.2 \%)$ ( $n=32$ ). Stapedial foramen large; sphenofrontal foramen and squamosal-alisphenoid groove present; large opening for the alisphenoid canal (primitive carotid and stapedial circulatory pattern 1; Voss, 1988). Alisphenoid strut absent, configuring oval accessory and buccinator-masticatory foramina confluent on a single foramen oval $(87.5 \%)$, or present, with distinct oval accessory and buccinator-masticatory foramina (symmetrically, in 3.1\%; and asymmetrically on the left side, $9.4 \%)(n=32)$. Suspensory squamosal process present and overlapped to the tegmen tympani, configuring a small postglenoid foramen. Subsquamosal fenestra very large. Stapedial process of ectotympanic present; external auditory meatus small and rounded; mastoid perforated by small fenestra. Incisive foramina moderately long, with lateral margins converging anteriorly $(56.25 \%)$, wider medially (convex) $(25 \%)$, or parallel $(18.75 \%)(n=32)$; posterior margin not reaching the anterior alveoli of M1 (96.9\%; reaching only in young specimens, $3.1 \%)(n=32)$. Posterior margin of zygomatic plate positioned nearly $(87.5 \%)$, anterior $(9,4 \%)$ or posterior $(3.1 \%)$ to the alveoli of $\mathrm{M} 1(\mathrm{n}=32)$. Palate wide and long, with two shallow lateral depression aligned with posterior palatine foramina; palate with posterolateral palatal pits simple, not positioned in depressions; palatal excrescencies absent. Mesopterygoid fossa penetrating between $(96.9 \%)$ or just leveled (3.1\%) to molar series ( $n=32$ ); roof of mestopterygoid fossa perforated by small sphenopalatine vacuities, positioned anteriorly to the presphenoid-basisphenoid suture $(93.5 \%)$ or by large vacuities, along the presphenoid and basisphenoid $(6.5 \%)(n=31)$. Parapterygoid fossa with the same width (44.8\%), narrower (37.9\%) or wider (17.2\%) than the mesopterygoid fossa $(n=29)$. Auditory bullae small and moderately globose, with the periotic margin extending to the internal carotid canal. Mandible elongated, with ramus high (Fig. 49). Condyloid process robust and long. Coronoid process developed, falciform, on the same level or lower than the condyloid process; angular process rounded, positioned posteriorly than condyloid process; superior and inferior notch moderately deep. Capsular process of lower incisor small and delicate (53.1\%) or absent $(46.9 \%)(n=32)$. Superior and inferior masseteric crests weakly developed, forming a single anterior crest (87.5\%) or converging in a V shape anteriorly (12.5\%) ( $n=32$ ). Upper incisors opisthodont. Upper molar parallel or slightly divergent posteriorly; molar series moderately long and wide; molars with main cusps arranged in opposite pairs; main cusps moderately low, united by conspicuous enamel (or dentine) flexus; dentine exposition moderate; labial and lingual flexus conspicuous, deeply overlapping. M1 with procingulum divided by deep anteromedian flexus; labial anteroconule equal in size to lingual one; anteroloph well developed; anteroflexus present; mesoloph robust, fused to mesostyle; mesoflexus very short; protostyle present; enterostyle absent; posteroloph present. $\mathrm{m} 1$ with procingulum developed, divided by a deep the anteromedian flexid or partially divided by an internal fold of the procingulum; anterolophid present; mesolophid developed; proto- stylid and protolophid present; small; ectostylid and ectolophid present.

Ontogenetic and sexual variation: We tested for ontogenetic variation using the 25 cranio-dental variables of specimens divided into four ACs. The ANOVA followed by Tukey post-hoc test, demonstrated that there is significant variation in 21 of the 25 variables tested (Table 40). Only the variables LM, LIB, LPB and IPL failed to vary significantly with age. The strongest divergences were found between classes $A C 1$ and $A C 2$ (17 variables with significant differences) and between classes AC2 and AC3 (18 variables with significant differences); between classes AC3 and AC4 only one variable was significantly different, suggesting that growth stabilizes from class $A C 3$. Based on these results, individuals in classes $A C 3$ and AC4 were clustered, and treated as adults. In these two classes, sexual dimorphism was evaluated by the t-student test on each of the 25 cranio-dental variables. Only incisive depth (ID) was significantly different between the sexes (Table 41). Studding morphological variation in D. dorsalis, Voss (1993) did not comment on ontogenetic or sexual variation of cranio-dental variables, however, he reported that the external morphology of the species varies with age - particularly in features of the dorsal pelage - and that secondary sexual dimorphism was not apparent. Our results corroborate the age differences and absence of sexual dimorphism in $D$. dorsalis as reported by Voss (1993).

Remarks: Comparing the EEB specimens of $D$. dorsalis with the descriptions provided by Gonçalves \& Oliveira (2014) we found high polymorphism in some characteristics mentioned by these authors as diagnostic for the species, for instance: the frontoparietal suture is not collinear with the frontosquamosal suture in $D$. dorsalis according to Gonçalves \& Oliveira (2014), however, this is very plastic in the EEB population, with some specimens exhibiting collinear sutures. Another example is the orientation of the lateral margins of the posterior portion of the incisive foramen, which are not curved according to Gonçalves \& Oliveira (2014), but, in EEB specimens this character is also highly polymorphic. On the other hand, some characteristics pointed out by Gonçalves \& Oliveira (2014) as diagnostic for $D$. dorsalis were consistent in the EEB specimens, such as: the angular process of the mandible pronounced and projected beyond the posterior limit of the condyloid process; the mesopterygoid fossa expanded only on its the anterior portion; and the bicolor pattern of tail coloration.

Field observations: The 52 specimens of $D$. dorsalis identified in the EEB samples were collected in December, 2003 (29 specimens), April, 2004 (21 specimens), and December, 2004 (two specimens). Pitfall traps captured thirty-eight individuals, while conventional traps captured 14 individuals (Sherman, Tomahawk and Museum Special). In December 2003, out of the 14 males, seven had scrotal testes and seven had abdominal testes; of the 13 females, nine were reproductively active (mam- 
Table 40. Results of Kolmogorov-Smirnov test, Analysis of Variance (ANOVA), and Tukey's post-hoc test, and sample size in each age class for 25 cranio-dental morphometric variables measured in Delomys dorsalis from EEB. Statistically significant values $(P<0.05)$ are indicated in bold.

\begin{tabular}{|c|c|c|c|c|c|c|c|c|c|c|c|c|c|}
\hline & \multirow{2}{*}{$\begin{array}{l}\text { K-S } \\
\text { Sig. }\end{array}$} & \multicolumn{2}{|c|}{ ANOVA } & \multicolumn{6}{|c|}{ Tukey } & \multicolumn{4}{|c|}{$\mathrm{N}$} \\
\hline & & $\mathbf{F}$ & Sig. & 1 vs. 2 & 1 vs. 3 & 1 vs. 4 & 2 vs. 3 & 2 vs. 4 & 3 vs. 4 & 1 & 2 & 3 & 4 \\
\hline GSL & 0.740 & 78.398 & 0.000 & 0.000 & 0.000 & 0.000 & 0.000 & 0.000 & 0.916 & 6 & 10 & 12 & 3 \\
\hline CIL & 0.570 & 97.661 & 0.000 & 0.000 & 0.000 & 0.000 & 0.000 & 0.000 & 0.951 & 6 & 10 & 12 & 2 \\
\hline CZL & 0.563 & 65.768 & 0.000 & 0.000 & 0.000 & 0.000 & 0.000 & 0.005 & 0.969 & 6 & 10 & 12 & 2 \\
\hline LD & 0.705 & 80.798 & 0.000 & 0.000 & 0.000 & 0.000 & 0.000 & 0.000 & 0.589 & 6 & 10 & 12 & 3 \\
\hline LIF & 0.889 & 49.125 & 0.000 & 0.000 & 0.000 & 0.000 & 0.000 & 0.002 & 0.935 & 6 & 10 & 12 & 3 \\
\hline BIF & 0.994 & 21.736 & 0.000 & 0.009 & 0.000 & 0.000 & 0.027 & 0.000 & 0.022 & 6 & 10 & 12 & 3 \\
\hline LM & 0.802 & 0.680 & 0.574 & - & - & - & - & - & - & 6 & 9 & 12 & 3 \\
\hline BM1 & 0.386 & 5.472 & 0.005 & 0.983 & 0.020 & 0.140 & 0.017 & 0.175 & 1.000 & 6 & 10 & 12 & 3 \\
\hline ID & 0.477 & 35.702 & 0.000 & 0.002 & 0.000 & 0.000 & 0.000 & 0.006 & 0.980 & 6 & 10 & 12 & 3 \\
\hline IW & 0.960 & 33.284 & 0.000 & 0.151 & 0.000 & 0.000 & 0.000 & 0.006 & 0.529 & 6 & 10 & 11 & 3 \\
\hline LN & 0.593 & 52.504 & 0.000 & 0.000 & 0.000 & 0.000 & 0.000 & 0.001 & 1.000 & 6 & 10 & 12 & 3 \\
\hline BR & 0.966 & 11.570 & 0.000 & 0.005 & 0.000 & 0.001 & 0.265 & 0.252 & 0.897 & 6 & 10 & 12 & 3 \\
\hline RL & 0.923 & 66.818 & 0.000 & 0.000 & 0.000 & 0.000 & 0.000 & 0.000 & 1.000 & 6 & 10 & 12 & 3 \\
\hline ZB & 0.929 & 34.336 & 0.000 & 0.005 & 0.000 & 0.000 & 0.000 & 0.005 & 0.999 & 6 & 10 & 11 & 2 \\
\hline BZP & 0.864 & 32.167 & 0.000 & 0.000 & 0.000 & 0.000 & 0.001 & 0.002 & 0.608 & 6 & 10 & 12 & 3 \\
\hline LIB & 0.519 & 2.548 & 0.077 & - & - & - & - & - & - & 6 & 10 & 12 & 3 \\
\hline OFL & 0.702 & 36.139 & 0.000 & 0.002 & 0.000 & 0.000 & 0.000 & 0.033 & 0.538 & 6 & 10 & 12 & 3 \\
\hline PL & 0.945 & 59.988 & 0.000 & 0.000 & 0.000 & 0.000 & 0.000 & 0.000 & 0.869 & 6 & 10 & 12 & 3 \\
\hline LPB & 0.620 & 2.332 & 0.097 & - & - & - & - & - & - & 6 & 10 & 12 & 3 \\
\hline BBP & 0.992 & 20.503 & 0.000 & 0.029 & 0.000 & 0.000 & 0.002 & 0.002 & 0.458 & 6 & 10 & 12 & 3 \\
\hline HBC & 0.848 & 9.609 & 0.000 & 0.210 & 0.000 & 0.015 & 0.012 & 0.236 & 0.995 & 6 & 10 & 12 & 3 \\
\hline BBC & 0.984 & 18.790 & 0.000 & 0.008 & 0.000 & 0.000 & 0.002 & 0.097 & 0.996 & 6 & 10 & 12 & 3 \\
\hline IPB & 0.975 & 4.051 & 0.017 & 0.795 & 0.036 & 0.997 & 0.133 & 0.790 & 0.092 & 6 & 10 & 12 & 3 \\
\hline IPL & 0.895 & 1.133 & 0.353 & - & - & - & - & - & - & 6 & 10 & 12 & 3 \\
\hline$O C B$ & 0.980 & 11.924 & 0.000 & 0.006 & 0.000 & 0.008 & 0.096 & 0.576 & 1.000 & 6 & 10 & 12 & 2 \\
\hline
\end{tabular}

mal glands visible and/or lactating, perforated vagina, or were pregnant) and four presented unperforated vaginas. In April, 2004, the reproductive condition of two out of 13 male specimens was examined and they had scrotal testes; of the seven females, two exhibited reproductive activity (mammal glands visible and/or lactating) and five were not apparently active (unperforated vagina). No information on the reproductive state of specimens captured in December, 2004, was obtained.

\section{Delomys sublineatus (Thomas 1903)}

Voucher material: $\mathrm{n}=31$; female: MZUSP 33717, 33718, 33750; uncatalogued: EBM 018, 250; EEB 699, 729, 874, 918, 1006, 1023; male: MZUSP 33720, 33721, 33725, 33727, 33771, 33773; uncatalogued: EBM 035, 091, 159, 232, 251, 304; EEB 556, 647, 771, 808, 844, 908, 1012, 1018.

Specimens identification: Large size (see body measurements on Table 39). Dorsal pelage coarse and not very dense, with heterogeneous coloration from dark brown to ochraceous yellow, and with a subtle longitudinal darker stripe from the scapular region to the rump; guard hairs long (length ranging from 10 to $11 \mathrm{~mm}$ ), with half proximal portion thinner and grayish and distal portion wider and with a basal band dark gray and apical band ochraceous brown; cover hairs moderately long (length ranging from 8.5 to $9.5 \mathrm{~mm}$ ), with proximal two thirds grayish and distal portion with a basal band ochraceous yellow and apical band brown; wool hairs numerous, shorter, wavy and thin (length ranging from 6 to $8 \mathrm{~mm})$, grayish colored with a subterminal band ochraceous yellow and a terminal band black. Lateral pelage dark brown more intensely grizzled with ochraceous yellow in young individuals and predominantly ochraceous yellow in adults; transition with ventral region well defined, clearly delimited by a yellowish line. Ventral pelage grayish, with hairs with basal half or basal third grayish and distal portion white. Four mammae pairs, in inguinal, abdominal, postaxial, and pectoral positions $(n=8)$. Mystacial vibrissae short, with few hairs reaching the base of pinnae when laid backwards. Pinnae small and rounded, weakly covered internally by yellowish hairs more ventrally and brown hairs more dorsally, and externally by brown hairs. Manus short and moderately narrow, covered dorsally by short hairs, with basal portion brown and apical white; manus with moderately long digits bearing very short claws (claw of dIII with length ranging from 0.8 to $1.2 \mathrm{~mm} ; \mathrm{n}=5$ ); dl vestigial, dll to $\mathrm{dV}$ longer, without conspicuous ungual tufts. Pes long and narrow, with dorsal surface covered by short hairs, with basal portion brown and apical white; pes with dl shorter, dIII equal or longer than dII and equal or shorter than dIV; digits with moderately long claws (claw of dIII with length ranging from 2.1 to $3.1 \mathrm{~mm} ; \mathrm{n}=5$ ); digits with sparse and moderately long ungual tufts, not completely concealing the claws; ungual tufts whitish, as well as digits; pes with moderately large interdigital pads; the- 
Table 41. Descriptive statistics (mean \pm standard deviation, and sample size) of 25 cranio-dental morphometric variables of adult specimens of Delomys dorsalis and Delomys sublineatus from EEB, separated by sex, and results of Student's t-test for sexual dimorphism and its value of significance. Statistically significant values $(\mathrm{P}<0.05)$ are indicated in bold.

\begin{tabular}{|c|c|c|c|c|c|c|c|c|}
\hline & \multicolumn{4}{|c|}{ Delomys dorsalis } & \multicolumn{4}{|c|}{ Delomys sublineatus } \\
\hline & Females & Males & $t$ & Sig. & Females & Males & $t$ & Sig. \\
\hline GSL & $33.75 \pm 0.87(9)$ & $34.30 \pm 1.04(6)$ & 1.109 & 0.287 & $32.57 \pm 0.79(3)$ & $33.38 \pm 1.69(6)$ & -0.773 & 0.465 \\
\hline CIL & $29.46 \pm 0.65(8)$ & $29.86 \pm 0.77(6)$ & -1.040 & 0.319 & $28.85 \pm 0.59(3)$ & $29.49 \pm 1.38(6)$ & -0.754 & 0.475 \\
\hline CZL & $21.54 \pm 0.44(8)$ & $22.06 \pm 0.77(6)$ & -1.599 & 0.136 & $21.73 \pm 0.48(3)$ & $22.34 \pm 0.98(6)$ & -0.988 & 0.356 \\
\hline LD & $9.15 \pm 0.23(9)$ & $9.27 \pm 0.46(6)$ & -0.667 & 0.516 & $8.54 \pm 0.13(3)$ & $8.68 \pm 0.72(7)$ & -0.314 & 0.761 \\
\hline LIF & $7.08 \pm 0.26(9)$ & $7.29 \pm 0.40(6)$ & -1.236 & 0.238 & $6.69 \pm 0.35(3)$ & $6.75 \pm 0.51(7)$ & -0.173 & 0.867 \\
\hline BIF & $2.65 \pm 0.16(9)$ & $2.69 \pm 0.24(6)$ & -0.416 & 0.684 & $2.61 \pm 0.06(3)$ & $2.43 \pm 0.20(7)$ & 1.132 & 0.295 \\
\hline LM & $4.82 \pm 0.07(9)$ & $4.80 \pm 0.19(6)$ & 0.267 & 0.794 & $4.64 \pm 0.22(3)$ & $4.75 \pm 0.17(7)$ & -0.837 & 0.427 \\
\hline BM1 & $1.53 \pm 0.07(9)$ & $1.48 \pm 0.07(6)$ & 1.424 & 0.178 & $1.46 \pm 0.09(3)$ & $1.41 \pm 0.04(7)$ & 1.062 & 0.319 \\
\hline ID & $1.49 \pm 0.08(9)$ & $1.42 \pm 0.03(6)$ & 2.431 & 0.030 & $1.46 \pm 0.04(3)$ & $1.44 \pm 0.12(7)$ & 0.390 & 0.707 \\
\hline IW & $1.77 \pm 0.11(8)$ & $1.70 \pm 0.07(6)$ & 1.500 & 0.160 & $1.66 \pm 0.08(3)$ & $1.65 \pm 0.11(6)$ & 0.167 & 0.872 \\
\hline LN & $13.44 \pm 0.67(9)$ & $13.95 \pm 0.53(6)$ & -1.556 & 0.144 & $12.97 \pm 0.72(3)$ & $13.83 \pm 1.09(6)$ & -1.222 & 0.261 \\
\hline BR & $4.71 \pm 0.35(9)$ & $4.71 \pm 0.13(6)$ & 0.011 & 0.991 & $4.79 \pm 0.33(3)$ & $4.41 \pm 0.11(7)$ & 2.944 & 0.019 \\
\hline RL & $13.94 \pm 0.46(9)$ & $14.39 \pm 0.67(6)$ & -1.561 & 0.142 & $13.15 \pm 0.24(3)$ & $13.33 \pm 1.02(6)$ & -0.283 & 0.786 \\
\hline ZB & $16.21 \pm 0.63(8)$ & $16.21 \pm 0.77(5)$ & -0.005 & 0.996 & $15.96 \pm 0.07(2)$ & $16.00 \pm 0.89(5)$ & -0.063 & 0.952 \\
\hline BZP & $2.98 \pm 0.14(9)$ & $2.95 \pm 0.25(6)$ & 0.241 & 0.814 & $2.84 \pm 0.20(3)$ & $2.90 \pm 0.20(7)$ & -0.429 & 0.680 \\
\hline LIB & $5.43 \pm 0.21(9)$ & $5.41 \pm 0.22(6)$ & 0.228 & 0.823 & $4.83 \pm 0.09(3)$ & $4.99 \pm 0.12(7)$ & -2.080 & 0.071 \\
\hline OFL & $10.00 \pm 0.38(9)$ & $10.20 \pm 0.25(6)$ & -1.100 & 0.291 & $10.09 \pm 0.44(3)$ & $10.44 \pm 0.34(6)$ & -1.331 & 0.225 \\
\hline PL & $13.39 \pm 0.46(9)$ & $13.43 \pm 0.42(6)$ & -0.173 & 0.865 & $12.80 \pm 0.30(3)$ & $12.75 \pm 0.72(7)$ & 0.095 & 0.926 \\
\hline LPB & $4.92 \pm 0.35(9)$ & $4.89 \pm 0.20(6)$ & 0.149 & 0.884 & $4.72 \pm 0.21(3)$ & $4.93 \pm 0.38(7)$ & -0.883 & 0.403 \\
\hline BBP & $3.34 \pm 0.21(9)$ & $3.30 \pm 0.24(6)$ & 0.398 & 0.697 & $3.17 \pm 0.04(2)$ & $3.12 \pm 0.25(7)$ & 0.514 & 0.624 \\
\hline HBC & $9.45 \pm 0.45(9)$ & $9.27 \pm 0.27(6)$ & 0.843 & 0.414 & $8.98 \pm 0.22(3)$ & $8.98 \pm 0.44(6)$ & -0.012 & 0.991 \\
\hline BBC & $14.14 \pm 0.53(9)$ & $13.94 \pm 0.28(6)$ & 0.826 & 0.424 & $13.32 \pm 0.12(3)$ & $13.39 \pm 0.44(6)$ & -0.281 & 0.787 \\
\hline IPB & $10.83 \pm 0.52(9)$ & $11.17 \pm 0.31(6)$ & -1.436 & 0.175 & $9.52 \pm 0.75(3)$ & $9.87 \pm 0.41(6)$ & -0.913 & 0.392 \\
\hline IPL & $3.85 \pm 0.29(9)$ & $4.03 \pm 0.38(6)$ & -1.014 & 0.329 & $3.41 \pm 0.29(3)$ & $3.80 \pm 0.38(6)$ & -1.547 & 0.166 \\
\hline$O C B$ & $7.35 \pm 0.37(8)$ & $7.33 \pm 0.09(6)$ & 0.166 & 0.872 & $7.05 \pm 0.16(3)$ & $7.05 \pm 0.31(7)$ & 0.040 & 0.969 \\
\hline
\end{tabular}

nar and hypothenar pads present; plantar integument between pads covered by squamae; pads flesh colored. Tail shorter than head and body $(84.1 \% \pm 7.4$ of head and body length, ranging from 70 to $96 \% ; n=18$ ); tail covered by short hairs, whose length equals the length of one to two scale rows on dorsal and ventral surface; apical tuft of hairs absent; scales small and arranged in 15 to 21 transverse rows per centimeter at the base of the tail, between 15 to 19 rows per $\mathrm{cm}$ on the central portion of the tail, and between 17 to 23 rows on the apical centimeter of the tail $(n=5)$; tail brownish dorsally (hairs entirely brown), light brownish ventrally (hairs with brown base and white apex, more concentrated on the base of the tail).

Skull long and narrow (Fig. 50; Table 39). Rostrum very long and narrow; rostral tube present and long; anterior portion of premaxillae and nasals projected anteriorly. Nasals long, not expanded laterally distally, with anterior margin projected beyond incisors and posterior margin (nasofrontal suture) surpassing (31\%) or not $(69 \%)$ the maxillar-frontal-lacrimal sutures $(n=29)$. Nasofrontal suture rounded. Premaxillae short, with caudal margin anterior (75.9\%) to or leveled (24.1\%) to the nasals $(n=29)$. Lacrimal equally in contact with the maxillar and frontal $(88.9 \%)$ or predominantly in contact with maxillar $(7.4 \%)$ or frontal $(3.7 \%)(n=27)$. Zygomatic notch moderately deep to deep, and narrow. Interorbital region symmetrically constricted (hourglass), with rounded (86.2\%) or sharp, squared (13.8\%; especially more posteriorly) su- praorbital margins ( $n=29$ ); supraorbital margins continuous with the moderately sharp temporal margin. Frontosquamosal suture collinear $(34.5 \%)$ or not $(65.5 \%)$ with the frontoparietal suture $(n=29)$. Zygomatic arches delicate, projected laterally, wider at the squamosal root. Braincase small and rounded. Parietals predominantly present $(96.4 \%)$ or exclusively present $(3.6 \%)$ on the dorsal surface of skull $(n=28)$. Interparietal long and wide. Rostrum with gnathic process very short; rostral tube present and long. Zygomatic plate moderately wide, with anterior margin straight or very slightly concave; free dorsal margin projected anteriorly, with rounded and downward sloping anterodorsal margin (62.1\%) or projected anteriorly with distinct anterior process (37.9\%) $(n=29)$. Zygomatic arches moderate, with jugal large with maxillar and squamosal processes not overlapping (89.3\%) or jugal small, with process overlapped, but not in contact $(10.7 \%)(n=28)$. Stapedial foramen large; sphenofrontal foramen and squamosal-alisphenoid groove present; large opening for the alisphenoid canal (primitive carotid and stapedial circulatory pattern 1; Voss, 1988). Alisphenoid strut absent, configuring oval accessory and buccinator-masticatory foramina confluent on a single foramen oval. Suspensory squamosal process present and overlapped to the tegmen tympani, configuring a small postglenoid foramen. Subsquamosal fenestra very large. Stapedial process of ectotympanic present; external auditory meatus small and rounded. Mastoid perforated by small fenestra. Incisive foramina 

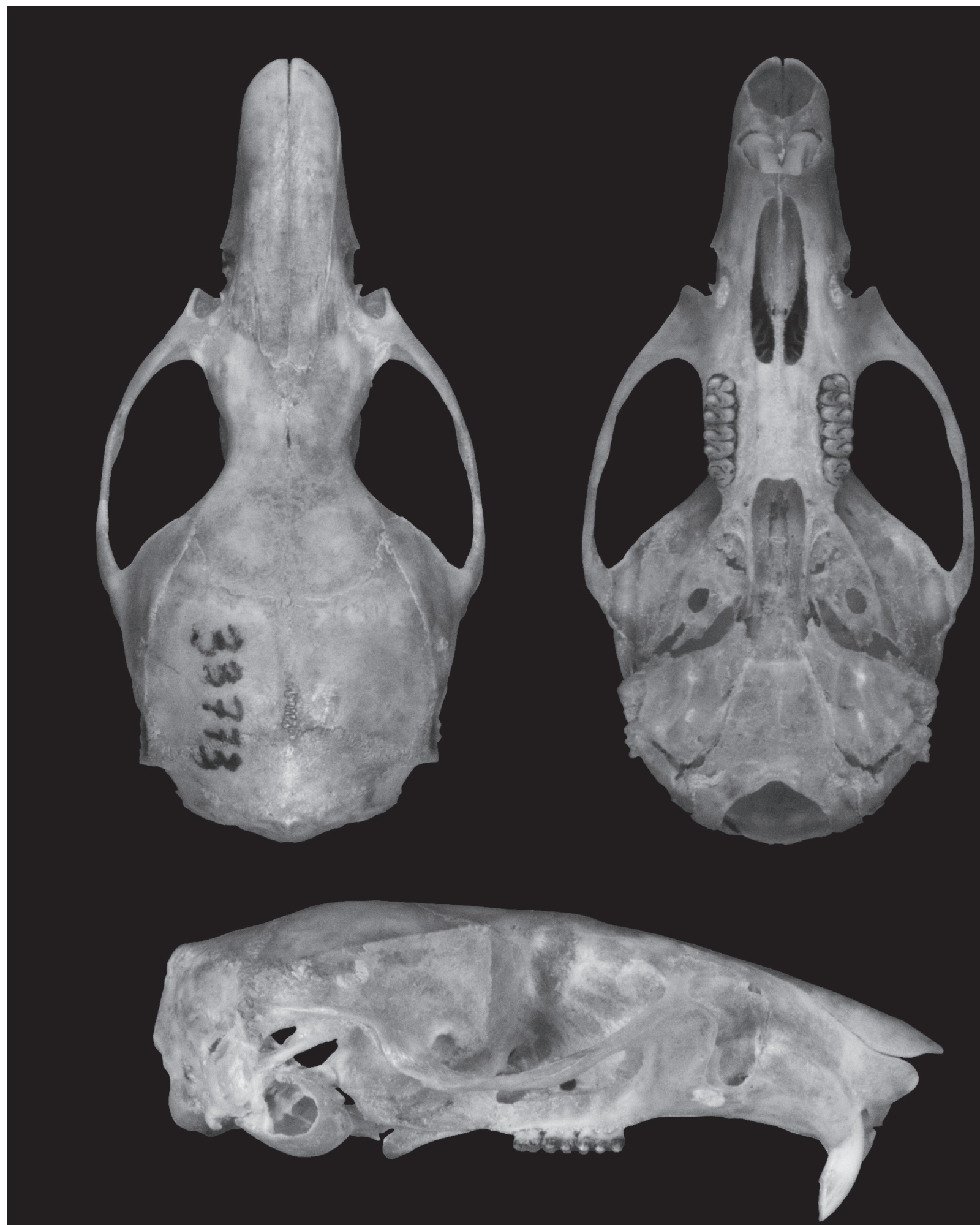

$5 \mathrm{~mm}$

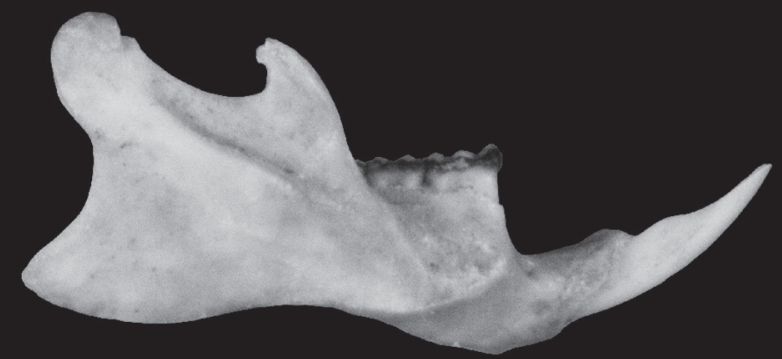

Figure 50. Skull and mandible of Delomys sublineatus (MZUSP 33773; male; GSL=33.64 mm). Skull on dorsal, ventral, and lateral view, and mandible on lateral view. 
moderately long, with lateral margins converging anteriorly $(46.4 \%)$, wider medially (convex) $(42.9 \%)$, or parallel $(10.7 \%)(n=28)$; posterior margin not reaching the anterior alveolus of M1 (93.1\%; reaching only in young specimens, $6.9 \%)(n=29)$. Posterior margin of zygomatic plate positioned nearly (69\%) or anterior $(31 \%)$ to the alveoli of M1 $(n=29)$. Palate wide and long, with two shallow lateral depression aligned with posterior palatine foramina; palate with posterolateral palatal pits simple $(65.5 \%)$ or double $(31 \%)$ on the palate level, or multiple, recessed in deep depressions (3.5\%) ( $n=29)$; palatal excrescencies absent. Mesopterygoid fossa penetrating between (96.6\%) or just leveled (3.4\%) to molar series $(n=29)$; roof of mestopterygoid fossa perforated by small sphenopalatine vacuities, positioned anteriorly to the presphenoid-basisphenoid suture (92.9\%) or by large vacuities, along the presphenoid and basisphenoid $(7.1 \%)(n=28)$. Parapterygoid fossa with the same width (57.7\%), narrower (11.5\%) or wider $(30.8 \%)$ than the mesopterygoid fossa $(n=29)$. Auditory bullae small and moderately globose, with the periotic margin extending to the internal carotid canal. Mandible elongated, with ramus high (Fig. 50). Condyloid process robust and long. Coronoid process developed, falciform, on the same level or lower than the condyloid process; angular process rounded, positioned posteriorly than condyloid process; superior and inferior notch moderately deep. Capsular process of lower incisor small and delicate $(24.1 \%)$ or absent $(75.9 \%)(n=29)$. Superior and inferior masseteric crests weakly developed, forming a single anterior crest (89.7\%) or converging in a V shape anteriorly (10.3\%) ( $n=29$ ). Upper incisors opisthodont. Upper molar parallel or slightly divergent posteriorly; molar series moderately long and wide; molars with main cusps arranged in opposite pairs; main cusps moderately low, united by conspicuous enamel (or dentine) flexus; dentine exposition moderate; labial and lingual flexus conspicuous, deeply overlapping. M1 with procingulum divided by deep anteromedian flexus, slightly asymmetric; labial anteroconule slightly larger than lingual one; anteroloph well developed; anteroflexus present; mesoloph robust, fused to mesostyle; mesoflexus very short; protostyle present; enterostyle absent; posteroloph present. $\mathrm{m} 1$ with procingulum developed, divided by a deep the anteromedian flexid or partially divided by an internal fold of the procingulum; anterolophid present; mesolophid developed; protostylid and protolophid present; small; ectostylid and ectolophid present.

Ontogenetic and sexual variation: We verified the ontogenetic variation in 25 cranio-dental variables of specimens of $D$. sublineatus divided into three ACs (AC1, AC2 and AC3). The ANOVA followed by Tukey post-hoc test, showed differences in 21 of the 25 variables tested (Table 42); the variables LM, LIB, LPB and IPB did not vary significantly throughout the species development (similarly to $D$. dorsalis). Between classes $A C 1$ and $A C 2,16$ variables were significantly different and between AC2 and $A C 3,15$ variables were significantly different; it was not possible to test for variation between AC4 and AC5,
Table 42. Results of Kolmogorov-Smirnov test, Analysis of Variance (ANOVA), and Tukey's post-hoc test, and sample size in each age class for 25 cranio-dental morphometric variables measured in Delomys sublineatus from EEB. Statistically significant values $(P<0.05)$ are indicated in bold.

\begin{tabular}{l|c|cc|ccc|ccc}
\hline & K-S & \multicolumn{2}{|c|}{ ANOVA } & \multicolumn{3}{c|}{ Tukey } & \multicolumn{3}{c}{ N } \\
\cline { 2 - 9 } & Sig. & F & Sig. & $\mathbf{1}$ vs.2 & $\mathbf{1}$ vs.3 & $\mathbf{2}$ vs.3 & $\mathbf{1}$ & $\mathbf{2}$ & $\mathbf{3}$ \\
\hline GSL & 0.931 & $\mathbf{4 5 . 9 1 2}$ & $\mathbf{0 . 0 0 0}$ & $\mathbf{0 . 0 0 0}$ & $\mathbf{0 . 0 0 0}$ & $\mathbf{0 . 0 0 0}$ & 5 & 14 & 9 \\
CIL & 0.828 & $\mathbf{5 6 . 4 6 9}$ & $\mathbf{0 . 0 0 0}$ & $\mathbf{0 . 0 0 0}$ & $\mathbf{0 . 0 0 0}$ & $\mathbf{0 . 0 0 0}$ & 5 & 14 & 9 \\
CZL & 0.998 & $\mathbf{4 9 . 8 9 7}$ & $\mathbf{0 . 0 0 0}$ & $\mathbf{0 . 0 0 0}$ & $\mathbf{0 . 0 0 0}$ & $\mathbf{0 . 0 0 0}$ & 5 & 14 & 9 \\
LD & 0.945 & $\mathbf{3 8 . 8 2 8}$ & $\mathbf{0 . 0 0 0}$ & $\mathbf{0 . 0 0 0}$ & $\mathbf{0 . 0 0 0}$ & $\mathbf{0 . 0 0 0}$ & 5 & 14 & 10 \\
LIF & 0.903 & $\mathbf{3 7 . 3 3 5}$ & $\mathbf{0 . 0 0 0}$ & $\mathbf{0 . 0 0 0}$ & $\mathbf{0 . 0 0 0}$ & $\mathbf{0 . 0 0 8}$ & 5 & 14 & 10 \\
BIF & 0.782 & $\mathbf{5 . 5 4 7}$ & $\mathbf{0 . 0 1 0}$ & 0.120 & $\mathbf{0 . 0 0 8}$ & 0.183 & 5 & 14 & 9 \\
LM & 0.387 & 1.894 & 0.171 & - & - & - & 5 & 14 & 10 \\
BM1 & 0.598 & $\mathbf{3 . 4 7 2}$ & $\mathbf{0 . 0 4 6}$ & 0.277 & $\mathbf{0 . 0 3 8}$ & 0.317 & 5 & 14 & 10 \\
ID & 0.967 & $\mathbf{2 3 . 5 8 0}$ & $\mathbf{0 . 0 0 0}$ & $\mathbf{0 . 0 0 0}$ & $\mathbf{0 . 0 0 0}$ & $\mathbf{0 . 0 0 5}$ & 5 & 14 & 10 \\
IW & 0.738 & $\mathbf{1 0 . 3 5 0}$ & $\mathbf{0 . 0 0 1}$ & $\mathbf{0 . 0 3 6}$ & $\mathbf{0 . 0 0 0}$ & $\mathbf{0 . 0 3 5}$ & 5 & 14 & 9 \\
LN & 0.975 & 33.735 & $\mathbf{0 . 0 0 0}$ & $\mathbf{0 . 0 0 0}$ & $\mathbf{0 . 0 0 0}$ & $\mathbf{0 . 0 0 1}$ & 5 & 14 & 9 \\
BR & 0.844 & 4.986 & $\mathbf{0 . 0 1 5}$ & $\mathbf{0 . 0 2 6}$ & $\mathbf{0 . 0 1 5}$ & 0.876 & 5 & 14 & 10 \\
RL & 0.965 & $\mathbf{4 0 . 9 1 5}$ & $\mathbf{0 . 0 0 0}$ & $\mathbf{0 . 0 0 0}$ & $\mathbf{0 . 0 0 0}$ & $\mathbf{0 . 0 0 0}$ & 5 & 14 & 9 \\
ZB & 0.919 & $\mathbf{1 5 . 7 7 0}$ & $\mathbf{0 . 0 0 0}$ & $\mathbf{0 . 0 1 9}$ & $\mathbf{0 . 0 0 0}$ & $\mathbf{0 . 0 0 4}$ & 4 & 13 & 7 \\
BZP & 0.606 & $\mathbf{4 0 . 1 7 1}$ & $\mathbf{0 . 0 0 0}$ & $\mathbf{0 . 0 0 0}$ & $\mathbf{0 . 0 0 0}$ & $\mathbf{0 . 0 2 0}$ & 5 & 14 & 10 \\
LIB & 0.908 & 0.187 & 0.831 & - & - & - & 5 & 14 & 10 \\
OFL & 0.952 & $\mathbf{2 9 . 7 1 4}$ & $\mathbf{0 . 0 0 0}$ & $\mathbf{0 . 0 0 0}$ & $\mathbf{0 . 0 0 0}$ & $\mathbf{0 . 0 0 3}$ & 5 & 14 & 9 \\
PL & 0.837 & $\mathbf{3 7 . 7 7 0}$ & $\mathbf{0 . 0 0 0}$ & $\mathbf{0 . 0 0 0}$ & $\mathbf{0 . 0 0 0}$ & $\mathbf{0 . 0 0 1}$ & 5 & 14 & 10 \\
LPB & 0.776 & 2.882 & 0.074 & - & - & - & 5 & 14 & 10 \\
BBP & 0.781 & $\mathbf{1 4 . 0 0 4}$ & $\mathbf{0 . 0 0 0}$ & $\mathbf{0 . 0 2 5}$ & $\mathbf{0 . 0 0 0}$ & $\mathbf{0 . 0 0 7}$ & 5 & 14 & 9 \\
HBC & 0.856 & $\mathbf{1 3 . 4 4 7}$ & $\mathbf{0 . 0 0 0}$ & 0.061 & $\mathbf{0 . 0 0 0}$ & $\mathbf{0 . 0 0 4}$ & 5 & 14 & 9 \\
BBC & 1.000 & $\mathbf{6 . 0 5 4}$ & $\mathbf{0 . 0 0 7}$ & 0.080 & $\mathbf{0 . 0 0 5}$ & 0.198 & 5 & 14 & 9 \\
IPB & 0.957 & 2.945 & 0.071 & - & - & - & 5 & 14 & 9 \\
IPL & 0.994 & $\mathbf{5 . 0 4 2}$ & $\mathbf{0 . 0 1 4}$ & $\mathbf{0 . 0 2 6}$ & $\mathbf{0 . 0 1 5}$ & 0.856 & 5 & 14 & 9 \\
OCB & 0.660 & $\mathbf{5 . 8 2 2}$ & $\mathbf{0 . 0 0 8}$ & 0.188 & $\mathbf{0 . 0 0 7}$ & 0.102 & 5 & 14 & 10 \\
\hline & & & & & & & &
\end{tabular}

since individuals within these classes were not available in the sample. Sexual dimorphism was tested in adult specimens (AC3), using the t-student test. Significant difference was present only in the breadth of rostrum (BR) (Table 41). Voss (1993) also studied the variation in the external morphology of this species and he found significant ontogenetic variation - particularly in the color of the pelage - and no indication of secondary sexual dimorphism, consistent with our results.

Field observations: A total of 31 specimens of $D$. sublineatus were identified among the specimens collected at EEB. They were collected in December, 2003 (14 specimens), January, 2004 (one specimen), April, 2004 (eight specimens), May, 2004 (one specimen), December, 2004 (three specimens), and December, 2011 (four specimens). Twenty-three individuals were captured in pitfall traps and eight in conventional traps (Sherman, Tomahawk and Museum Special). In December, 2003, five males had scrotal testes, three females were reproductively active (with mammary glands visible and perforated vaginas and/or pregnant) and one female had unperforated vagina. In April, 2004, one male with scrotal testes, one female with perforated vagina and mammary glands visible and one female with unperforated vagina were collected. In December, 2011, only one female had mammary glands developed. 


\section{Family Echimyidae \\ Subfamily Echimyinae \\ Genus Phyllomys}

Two species of Phyllomys occur at EEB, P. kerri and $P$. sulinus, which were recognized in a previous study (Abreu-Júnior et al., 2018) based on molecular and morphological data. Phyllomys kerri is represented at EEB by two juvenile specimens with skin and skull preserved, and $P$. sulinus is represented by one adult specimen with skin preserved, but skull missing. On the external morphology, according to Abreu-Júnior et al. (2018), these species can be distinguished by the texture of dorsal pelage, that is spiny with wide aristiforms on rump (about $1.0 \mathrm{~mm}$ ) in $P$. kerri and varies from soft to stiff with narrow aristiforms on rump (about $0.4 \mathrm{~mm}$ ) in $P$. sulinus; and also, by the tail hairs that are mostly pale brown in $P$. kerri and dark brow in $P$. sulinus.

\section{Phyllomys kerri (Moojen 1950)}

Voucher material: $\mathrm{n}=2$; female: uncatalogued: $E E B$ 751; male: uncatalogued: EEB 734.

Specimens identification: Large size (see body measurements on Table 43). Dorsal pelage long and spiny, but not very rigid, grayish brown colored speckled with light orange; dorsal guard hairs long (ranging from 24 to $32 \mathrm{~mm}$ long), thin at the apical extremity (whip-like tip) and wider at the base, light gray colored at basal portion $(2 / 3)$ and gradually getting dark gray to the top; dorsal cover hairs long (length ranging from 20 to $24 \mathrm{~mm}$ ) and thin, grayish colored with a subterminal band orange and a terminal band black; dorsal wool hairs short (length ranging from 12 to $15 \mathrm{~mm}$ ) and thin, grayish colored. Lateral pelage grayish brown speckled with orange; limit between lateral and ventral color well defined. Ventral pelage heterogeneous, cream or grayish cream, with some white spots; individual ventral hairs entirely cream or grayish cream, or even white. Mystacial vibrissae with numerous and long hairs, which surpass the top of the pinnae when laid back. Pinnae small and covered by dark hairs; presence of a tuft of dark hairs coming from the base of the pinnae, about twice the length of the pinnae. Dorsal surface of manus covered by numerous hairs, mostly light brow colored, but some grayish colored; digits with medium claws (claw of dlll with length ranging from 3 to $3.5 \mathrm{~mm} ; \mathrm{n}=2$ ). Pes moderately long and wide, dorsally covered by numerous hairs, mostly grayish light brow colored; dIII similar in length to IV and longer than dll; digits with long claws (claw of dIII = $3.7 \mathrm{~mm} ; \mathrm{n}=2$ ); tufts of ungual hairs short, not extending beyond the claws, grayish colored. Tail with length similar to head and body (ranging from 96.5 to $101.3 \%$ of head and body length; $n=2$ ); rump hairs covering about $1 / 7$ of the basal portion of the tail; tail covered by long hairs, with length of five to six scales on both surfaces; scales arranged in 18 to 21 transverse rows per centimeter at the base and central portion of the tail, and between 23 and 27 rows
Table 43. Body and cranio-dental morphometric values $(\mathrm{mm})$, and weight $(\mathrm{g})$ of young specimens of Phyllomys kerri from EEB.

\begin{tabular}{|c|c|c|}
\hline & EEB 734 (male) & EEB 751 (female) \\
\hline $\mathrm{HBL}$ & 172.0 & 158.0 \\
\hline TL & 166.0 & 160.0 \\
\hline HF1 & 33.0 & 33.0 \\
\hline HF2 & 36.0 & 34.0 \\
\hline EL & 15.0 & 15.0 \\
\hline W & 130.0 & 95.0 \\
\hline GSL & 44.32 & 38.80 \\
\hline CIL & 38.16 & 33.48 \\
\hline LD & 9.16 & 7.57 \\
\hline LIF & 4.41 & 4.16 \\
\hline BIF & 1.50 & 1.84 \\
\hline PL & 16.12 & 13.87 \\
\hline LN & 12.56 & 11.48 \\
\hline RL & 15.26 & 12.35 \\
\hline OFL & 12.77 & 11.95 \\
\hline BR & 4.77 & 4.77 \\
\hline LIB & 9.21 & 8.15 \\
\hline ZB & 19.66 & 19.02 \\
\hline MB & 17.20 & 15.22 \\
\hline DBB & 3.94 & 3.22 \\
\hline$O C B$ & 8.56 & 8.31 \\
\hline BL & 9.43 & 9.10 \\
\hline $\mathrm{HBC}$ & 12.78 & 13.55 \\
\hline HCM1 & - & 11.69 \\
\hline HR & 8.72 & 8.17 \\
\hline HZA & 4.84 & 4.43 \\
\hline
\end{tabular}

on the apical centimeter of the tail $(n=2)$; short tuft of numerous hairs at the tail tip, brownish colored; dorsal and ventral tail color pale brown, with pale brown hairs and some whitish hairs on ventral surface.

Skull robust and elongated (Fig. 51; Table 43). Rostrum short, with rostral tube present. Nasals anteriorly long, extending beyond the superior incisors, and posteriorly short, not extending beyond the maxillary-frontal-lacrimal suture. Premaxillae posteriorly long, extending beyond the nasals. Interorbital region divergent posteriorly, with supraorbital crests laterally expanded. Braincase large and elongated. Parietal largely expanded to the lateral surface of braincase. Foramen nasolacrimal high, ovate and vertically oriented or rounded. Post-orbital process of the zygoma rounded, formed by squamosal and jugal. Alisphenoid strut present and large; foramen buccinator and masticatory not confluent. Foramen oval rounded or elongated. Posterior opening of alisphenoid canal invisible on lateral surface of skull. Mastoid process short, not surpassing the midline of the external auditory meatus. Incisive foramina generally oval in shape. Mesopterygoid fossa "V" shaped, with the anterior portion slightly rounded and reaching the posterior surface of $\mathrm{M} 1$, or with the anterior portion sharped and reaching the middle of M2. Sphenopalatine vacuities present as rounded openings, anteriorly to basisphenoid-presphenoid suture. Auditory bullae large. Mandible robust, with ramus deep (Fig. 51). Angular process long, surpassing the condylar process. Ventral root of the angular process laterally deflected. Superior masseteric ridge reduced and inferior well developed. Ventral mandibular spine 


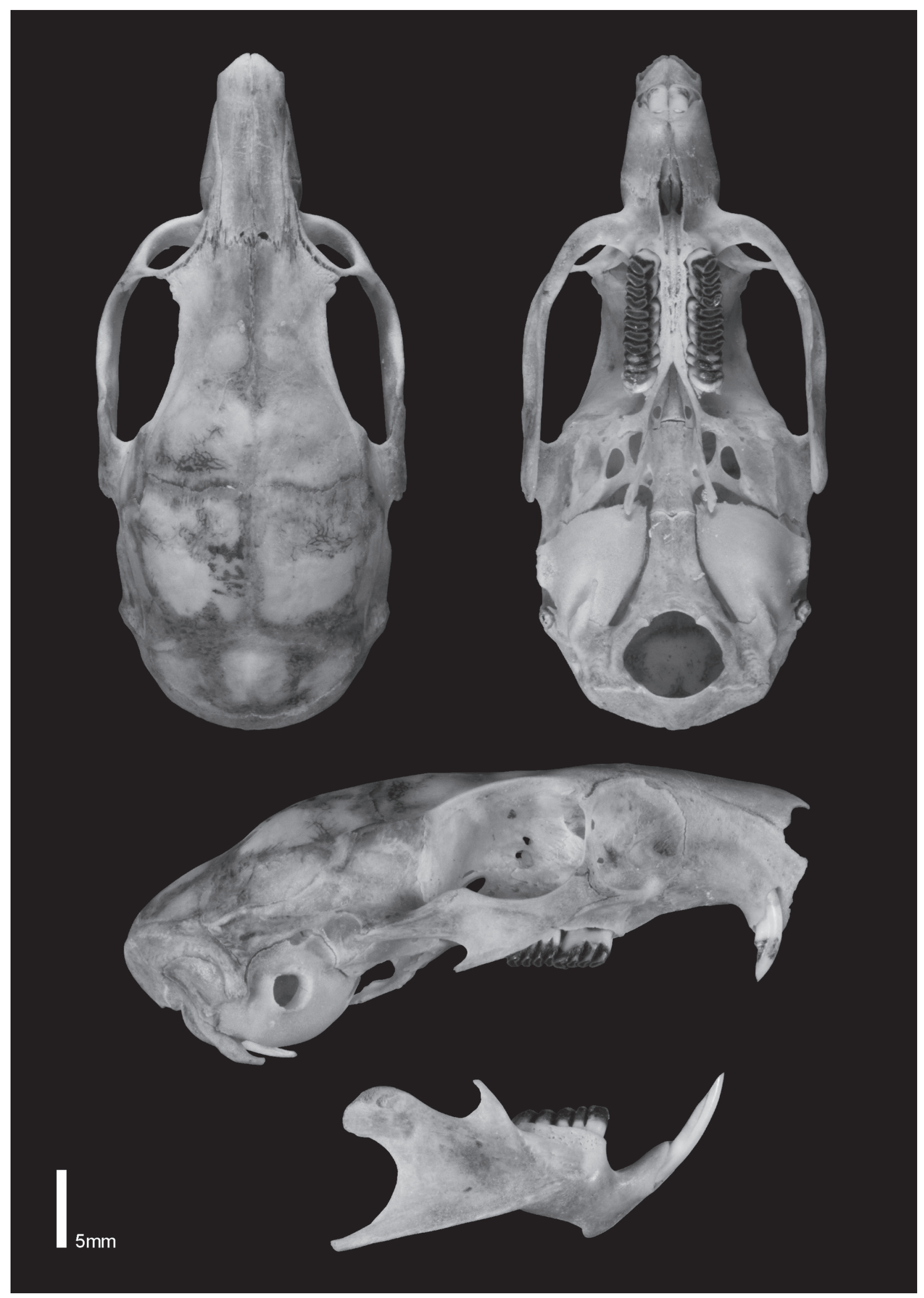

Figure 51. Skull and mandible of Phyllomys kerri (EEB 734; male; GSL = $44.32 \mathrm{~mm}$ ). Skull on dorsal, ventral, and lateral view, and mandible on lateral view. 
absent. Upper incisors slightly opisthodont. Maxillary toothrows incomplete in all specimens analyzed. Upper molars tetralophodont and lower molars trilophodont.

Field observations: Both specimens of $P$. kerri were captured in December, 2003, with pitfall traps. They were juveniles, one female and one male, without signs of reproductive activity.

\section{Phyllomys sulinus Leite, Christoff \& Fagundes 2008}

Voucher material: $\mathrm{n}=1$; sex unknown: uncatalogued: EBM 798.

Specimen identification: Large size. Dorsal pelage long and stiff, rusty orange speckled with black hairs; dorsal guard hairs long (length ranging from 27 to $32 \mathrm{~mm}$ ), thin at the apical extremity (whip-like tip) and wider at the base, light gray colored at basal portion ( $2 / 3)$ and gradually getting dark gray to the top; dorsal cover hairs long (length ranging from 24 to $28 \mathrm{~mm}$ ) and thin, grayish colored with a subterminal band orange and a terminal band black; dorsal wool hairs short (length about $13 \mathrm{~mm}$ ) and thin, grayish colored. Lateral pelage predominantly orangish; limit between lateral and ventral color strongly defined. Ventral pelage heterogeneous, predominantly whitish colored with grayish patches; individual ventral hairs generally with base white or gray, and with apical portion whitish or cream. Mystacial vibrissae with numerous and long hairs, which surpass the top of the pinnae when laid back. Pinnae small and covered by dark hairs; presence of a tuft of dark hairs coming from the base of the pinnae, about twice the length of the pinnae. Four mammae pairs, in inguinal, abdominal, pectoral, and postaxial positions. Dorsal surface of manus covered by numerous hairs, mostly grayish colored; digits with medium claws (claw of dIII = $3.75 \mathrm{~mm}$ ). Pes moderately long and wide, dorsally covered by numerous hairs, mostly grayish colored; dIII similar in length to IV and longer than dll; digits with long claws (claw of dIII $=4.5 \mathrm{~mm}$ ); tufts of ungual hairs long, extending beyond the claws, whitish colored. Tail with length similar to head and body; tail covered by long hairs, with length surpassing six scales on both surfaces; scales arranged in 14 transverse rows per centimeter at the base and central portion of the tail, and in 16 rows on the apical centimeter of the tail; tuft of numerous hairs at the tail tip (length about $10 \mathrm{~mm}$ ), brownish colored; dorsal tail color dark brown, with dark brown hairs, and ventral tail color light brown, with light brown hairs; tail slightly bicolor.

Field observations: No field observation is available for this species.

\section{Subfamily Eumysopinae} Euryzygomatomys spinosus (G. Fischer 1814)

Voucher material: $\mathrm{n}=1$; female: uncatalogued: EEB 818 .
Specimens identification: Large size (see body measurements on Table 44). Dorsal pelage dense and slightly rigid, grayish dark brown slightly speckled with ocher yellow; dorsal guard hairs rigid and long (length ranging from 20 to $22 \mathrm{~mm}$ ), with the basal portion wider (width about $0.4 \mathrm{~mm}$ ) and apical portion narrower, light gray colored ate the base and gradually getting dark to the top; dorsal cover hairs and wool hairs with same pattern, slightly smaller than guard hairs (length about $18 \mathrm{~mm}$ ), light gray colored at the basal half and the apical half with two inferior thirds dark gray and an apical third ocher yellow. Lateral pelage dark gray mixed with ocher yellow; limit between lateral and ventral color very sharply delimited. Ventral pelage color white, individual ventral hairs entirely white. Mystacial vibrissae short, not reaching the top of the pinnae when laid back. Pinnae small; covered internally and externally by sparse and short hairs, blackish colored. Dorsal surface of manus covered by predominantly grayish hairs; digits with very long claws (claw of dlll $=5 \mathrm{~mm} ; \mathrm{n}=1$ ). Pes short and wide, dorsally covered by predominantly grayish hairs; dIII longer than dII and dIV; digits with very long claws (claw of dIII $=5.5 \mathrm{~mm} ; \mathrm{n}=1$ ); tufts of ungual hairs sparse and short, usually not extending beyond the claws, whitish colored. Tail shorter than head and body; covered by hairs with length extending three scales at the basal portion on both surfaces; scales arranged in 19 transverse rows per centimeter at the base and at the top of the tail $(n=1)$; dorsal tail color dark brown, with dark brown hairs, and ventral tail color light brown, with slightly yellowish hairs.

Skull very robust, short and wide (Fig. 52; Table 44). Rostrum very short. Nasals anteriorly short, not extending beyond the superior incisors, and posteriorly also short, not extending beyond the maxillary-frontal-lacrimal suture. Premaxillae posteriorly long, extending beyond the nasals. Interorbital region divergent posteriorly, with low crests, expanded mainly laterally. Braincase large, wide and slightly elongated. Parietal largely expanded to the lateral surface of braincase. Foramen nasolacrimal high and rounded. Post-orbital process of the zygoma not prominent, formed by jugal and squamosal. Foramen buccinator and masticatory absent. Alisphenoid strut absent. Foramen oval large and ovoid. Mastoid process long, surpassing the midline of the external auditory meatus. Incisive foramina short and wide, rounded in shape; incisive foramina septum formed only by premaxillary. Mesopterygoid fossa "V" shaped, surpassing the center of M2. Sphenopalatine vacuities present as large openings along the presphenoid reaching basisphenoid. Auditory bullae large. Mandible robust, with ramus deep (Fig. 52). Angular process long and narrow, surpassing the condylar process. Superior masseteric ridge reduced and inferior well developed. Ventral mandibular spine absent. Upper incisors proodont. Maxillary toothrows anteriorly convergent. Upper and lower molars trilophodont.

Remarks: Euryzygomatomys spinosus is among the least known echimid rodents from AF, possibly because of 
Table 44. Body and cranio-dental morphometric values $(\mathrm{mm})$, and weight $(\mathrm{g})$ of Euryzygomatomys spinosus from Atlantic Forest localities ( ${ }^{*}$ EEB; ${ }^{* *}$ Villarica, Paraguay; ${ }^{* * *}$ Casa Grande, São Paulo State, Brazil), and descriptive statistics (mean \pm standard deviation, range, and sample size) of young and adult specimens from Itapetininga, São Paulo State.

\begin{tabular}{|c|c|c|c|c|c|}
\hline & EEB 818* & AMNH $66785^{* *}$ & USNM $461067^{* * *}$ & Itapetininga (Youngs) & Itapetininga (Adults) \\
\hline Sex & male & - & female & 2 males & 3 males and 2 females \\
\hline Age class & 5 & 7 & 7 & $1-4$ & $9-10$ \\
\hline HBL & - & - & 188.0 & $(137.0-170.0) 2$ & $204.60 \pm 10.24(190.0-216.0) 5$ \\
\hline TL & - & - & 48.0 & $(41.0-43.0) 2$ & $53.67 \pm 8.62(46.0-63.0) 3$ \\
\hline HF2 & - & - & 30.0 & $(26.0-30.0) 2$ & $33.20 \pm 2.49(30.0-37.0) 5$ \\
\hline EL & - & - & 20.0 & (16.0) 2 & $18.00 \pm 2.12(15.0-20.0) 5$ \\
\hline w & - & - & - & - & - \\
\hline GSL & 39.90 & 43.45 & 44.44 & $(33.17-39.27) 2$ & $44.01 \pm 1.23(42.69-45.39) 5$ \\
\hline CIL & 37.51 & 41.83 & 40.74 & $(30.42-37.19) 2$ & $42.09 \pm 1.04(40.72-43.27) 5$ \\
\hline LD & 9.37 & 10.50 & 10.32 & $(7.23-9.25) 2$ & $10.86 \pm 0.40(10.38-11.31) 5$ \\
\hline MAL & - & 9.27 & - & - & $9.52 \pm 0.18(9.24-9.69) 5$ \\
\hline BPM & - & 2.42 & 2.47 & $(2.20-2.31) 2$ & $2.83 \pm 0.03(2.80-2.88) 5$ \\
\hline LIF & 4.18 & 5.11 & 4.04 & $(2.93-4.51) 2$ & $5.09 \pm 0.48(4.34-5.55) 5$ \\
\hline BIF & 2.81 & 2.72 & 2.55 & $(2.27-2.63) 2$ & $2.80 \pm 0.23(2.54-3.07) 5$ \\
\hline PL & 15.88 & 16.55 & 16.51 & $(11.13-15.74) 2$ & $18.22 \pm 0.77(17.12-18.97) 5$ \\
\hline LN & 11.39 & 12.64 & 13.52 & $(9.03-11.09) 2$ & $12.67 \pm 0.71(11.61-13.53) 5$ \\
\hline RL & 14.12 & 14.74 & 15.73 & $(10.57-13.61) 2$ & $16.03 \pm 0.45(15.47-16.52) 5$ \\
\hline OFL & 11.53 & 12.11 & 11.89 & $(9.55-10.92) 2$ & $12.18 \pm 0.16(12.05-12.39) 5$ \\
\hline BR & 7.20 & 7.64 & 7.60 & $(6.22-7.13) 2$ & $7.31 \pm 0.10(7.17-7.41) 5$ \\
\hline LIB & 10.01 & 9.78 & 11.36 & $(8.80-10.35) 2$ & $10.99 \pm 0.44(10.48-11.60) 5$ \\
\hline ZB & 23.81 & 25.71 & 26.94 & $(19.94-23.46) 2$ & $25.91 \pm 0.48(25.10-26.31) 5$ \\
\hline MB & 19.72 & 20.54 & 21.45 & 19.36 & $20.70 \pm 0.50(19.89-21.21) 5$ \\
\hline PW & - & 8.17 & - & - & $9.34 \pm 0.71(8.56-10.0) 5$ \\
\hline DBB & 3.66 & 3.77 & 3.77 & $(2.72-3.19) 2$ & $4.25 \pm 0.27(3.90-4.63) 5$ \\
\hline OCB & 9.41 & 9.75 & 10.40 & $(7.11-8.66) 2$ & $8.83 \pm 0.25(8.57-9.17) 5$ \\
\hline BL & 10.74 & 10.80 & 11.60 & $(9.42-10.85) 2$ & $10.70 \pm 0.41(10.26-11.16) 5$ \\
\hline HBC & 14.86 & 13.09 & 14.08 & $(11.42-12.96) 2$ & $13.34 \pm 0.71(12.82-14.41) 5$ \\
\hline HCM1 & 13.47 & 14.28 & 15.20 & $(11.20-13.50) 2$ & $15.07 \pm 0.28(14.83-15.52) 5$ \\
\hline HR & 8.08 & 8.86 & 9.21 & $(7.10-8.22) 2$ & $9.20 \pm 0.14(9.01-9.39) 5$ \\
\hline HZA & 5.17 & 5.71 & 6.05 & $(4.40-6.06) 2$ & $6.33 \pm 0.40(5.76-6.88) 5$ \\
\hline
\end{tabular}

the small number of specimens available in collections, which could reflect a low capture rate for the species: in the EEB expeditions only one individual of $E$. spinosus was trapped among about 600 specimens. We compared body and cranio-dental morphometric values of E. spinosus from four AF localities (Table 44). The sample from Itapetininga, SP, is the largest one and for that values from juvenile and adult specimens are presented separately. The specimen from EEB, as a juvenile, presents morphometric values closely to those from juvenile specimens from Itapetininga.

Field observations: The only specimen of $E$. spinosus collected at EEB was a juvenile female, captured with pitfall trap in April, 2004.

Additional specimens examined: Euryzygomatomys spinosus: $\mathrm{n}=9$; Brasil: São Paulo: Casa Grande (2325'58.79"S, 4655'58.79"W): male: USNM 461067; Itapetininga $\left(23^{\circ} 33^{\prime} 34.99^{\prime \prime} \mathrm{S}, 48^{\circ} 3^{\prime} 11.16^{\prime \prime} \mathrm{W}\right)$ : male: USNM 460564, 462138, 462139, 462140, 462143; female: USNM 462141, 462144; Paraguay: Guaira: Villarica (254'55"S, $\left.56^{\circ} 25^{\prime} 59^{\prime \prime} \mathrm{W}\right)$ : sex unknown: AMNH 66785.

\section{Trinomys dimidiatus (Günther 1876)}

Voucher material: $\mathrm{n}=3$; female: uncatalogued: EEB 1011, 1013, 1024.

Specimens identification: Large size (see body measurements on Table 45). Dorsal pelage rigid, orange brown with darkish parts, mostly near the dorsal midline and the rump; dorsal guard hairs long (length ranging from 17 to $17.5 \mathrm{~mm}$ ), thin at extremities (whip-like tip) and wider at the middle (width ranging from 0.5 to $0.6 \mathrm{~mm}$ ), unpigmented at the basal portion and gradually getting dark gray to the top, with a subterminal black band and a brownish apical band; dorsal cover hairs medium (length ranging from 10 to $13 \mathrm{~mm}$ ), light gray colored at the base, gray at the middle and with a subterminal orange band and a gray apical band; dorsal wool hairs short (length ranging from 5 to $8.5 \mathrm{~mm}$ ), with the basal half wider, gray in color, and the apical half narrower, slightly brownish. Lateral pelage orange brown speckled with black; limit between lateral and ventral color sharply defined. Ventral pelage color white, individual ventral hairs entirely white. Mystacial vibrissae long, far surpassing the top of the pin- 

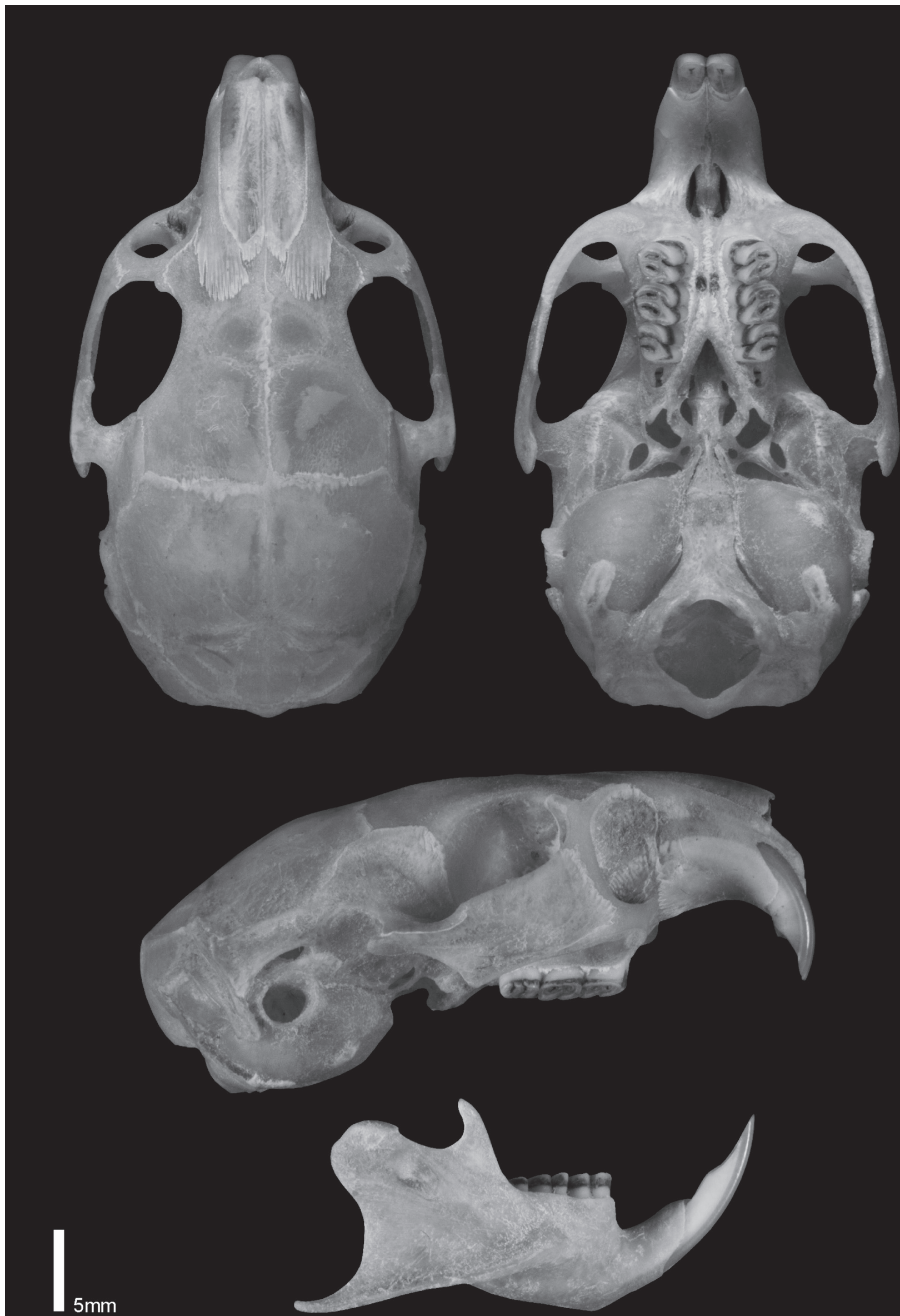

Figure 52. Skull and mandible of Euryzygomatomys spinosus (EEB 818; male; GSL=39.90 mm). Skull on dorsal, ventral, and lateral view, and mandible on lateral view. 
Table 45. Descriptive statistics (mean \pm standard deviation, range, and sample size) of body and cranio-dental measurements ( $\mathrm{mm}$ ), and weight ( $\mathrm{g}$ ), of adult specimens (AC 8-10) of Trinomys dimidiatus from EEB and localities in Rio de Janeiro State, and descriptive statistics of Trinomys iheringi from Casa Grande, São Paulo State.

\begin{tabular}{|c|c|c|c|}
\hline & \multicolumn{2}{|c|}{ T. dimidiatus } & \multirow{2}{*}{$\begin{array}{l}\text { T. iheringi } \\
\text { Casa Grande specimens ( } 6 \text { females, } 5 \text { males and } 1 \text { unknown) }\end{array}$} \\
\hline & EEB specimens (2 females) & RJ specimens (3 females) & \\
\hline HBL & $(201.0-208.0) 2$ & $(210.0-220.0) 2$ & $194.40 \pm 9.16(180.0-205.0) 10$ \\
\hline TL & $(177.0-179.0) 2$ & $178.33 \pm 7.64(170.0-185.0) 3$ & $164.89 \pm 13.61(135.0-185.0) 9$ \\
\hline HF1 & $(34.0-41.0) 2$ & - & - \\
\hline HF2 & $(37.0-44.0) 2$ & $50.00 \pm 2.00(48.0-52.0) 3$ & $41.80 \pm 1.40(40.0-44.0) 10$ \\
\hline EL & $(26.0) 2$ & (20.0) 2 & $23.80 \pm 1.03(22.0-25.0) 10$ \\
\hline W & $(170.0-180.0) 2$ & $(200.0-250.0) 2$ & - \\
\hline GSL & $(50.17-50.55) 2$ & $51.10 \pm 1.40(50.14-52.70) 3$ & $48.84 \pm 1.56(46.98-52.00) 12$ \\
\hline CIL & $(41.57-41.66) 2$ & $42.13 \pm 0.93(41.55-43.20) 3$ & $39.97 \pm 1.43(38.13-42.37) 12$ \\
\hline LD & $(10.88-11.26) 2$ & $10.96 \pm 0.84(10.16-11.84) 3$ & $10.63 \pm 0.54(9.89-11.77) 12$ \\
\hline MAL & $(7.76-8.01) 2$ & $8.33 \pm 0.35(7.97-8.67) 3$ & $7.84 \pm 0.21(7.52-8.20) 11$ \\
\hline BPM & $(2.18-2.30) 2$ & $2.15 \pm 0.06(2.09-2.19) 3$ & $1.94 \pm 0.11(1.73-2.19) 12$ \\
\hline LIF & $(3.29-3.84) 2$ & $3.68 \pm 0.17(3.48-3.80) 3$ & $3.84 \pm 0.33(3.30-4.51) 12$ \\
\hline BIF & $(2.17-2.51) 2$ & $2.47 \pm 0.06(2.44-2.54) 3$ & $2.38 \pm 0.14(2.11-2.59) 12$ \\
\hline PL & $(15.53-16.30) 2$ & $16.01 \pm 0.46(15.64-16.52) 3$ & $15.31 \pm 0.76(14.48-17.06) 12$ \\
\hline LN & $(19.24-19.70) 2$ & $18.99 \pm 0.86(18.42-19.98) 3$ & $17.26 \pm 0.76(16.16-18.82) 12$ \\
\hline RL & $(20.33-20.98) 2$ & $20.73 \pm 1.41(19.66-22.33) 3$ & $19.30 \pm 0.72(18.23-20.60) 12$ \\
\hline OFL & $(11.83-12.64) 2$ & $12.45 \pm 0.25(12.26-12.73) 3$ & $12.36 \pm 0.38(11.65-12.83) 12$ \\
\hline BR & $(7.40-749) 2$ & $7.57 \pm 0.50(7.06-8.05) 3$ & $7.01 \pm 0.35(6.57-7.87) 12$ \\
\hline LIB & $(11.23-11.42) 2$ & $11.73 \pm 0.15(11.61-11.90) 3$ & $10.78 \pm 0.34(10.37-11.35) 12$ \\
\hline ZB & $(25.24-25.41) 2$ & $25.73 \pm 0.47(25.37-26.26) 3$ & $24.74 \pm 0.72(23.73-26.59) 11$ \\
\hline MB & $(18.25-18.57) 2$ & $18.96 \pm 0.29(18.67-19.25) 3$ & $18.54 \pm 0.50(17.97-19.73) 12$ \\
\hline PW & $(9.25-9.43) 2$ & $(8.62-8.86) 2$ & $8.88 \pm 0.32(8.26-9.22) 10$ \\
\hline DBB & $(4.75-5.52) 2$ & $5.25 \pm 0.20(5.11-5.48) 3$ & $4.84 \pm 0.45(4.32-5.78) 12$ \\
\hline OCB & $(9.10-9.42) 2$ & $9.80 \pm 0.34(9.42-10.05) 3$ & $9.47 \pm 0.38(8.96-10.11) 12$ \\
\hline BL & $(9.21-9.45) 2$ & $9.00 \pm 0.23(8.74-9.19) 3$ & $8.87 \pm 0.41(8.03-9.42) 12$ \\
\hline HBC & $(15.20-15.39) 2$ & $13.94 \pm 0.53(13.33-14.34) 3$ & $13.45 \pm 0.57(13.07-14.20) 12$ \\
\hline HCM1 & $(13.87-15.98) 2$ & $13.62 \pm 0.29(13.34-13.92) 3$ & $13.32 \pm 0.34(12.92-14.08) 12$ \\
\hline HR & $(9.84-9.96) 2$ & $10.03 \pm 0.32(9.69-10.31) 3$ & $9.58 \pm 0.32(9.10-10.18) 12$ \\
\hline HZA & $(5.40-5.67) 2$ & $5.26 \pm 0.15(5.11-5.40) 3$ & $5.03 \pm 0.32(4.45-5.53) 12$ \\
\hline
\end{tabular}

nae when laid back. Pinnae large and oval shaped; covered internally and externally by very sparse and short hairs, almost imperceptible to naked eye; presence of a dark hairs tuft coming from the base of the pinnae. Dorsal surface of manus covered by numerous hairs, whitish colored; digits with short claws (claw of dIII $=2.6 \mathrm{~mm} ; \mathrm{n}=2$ ). Pes very long and narrow, dorsally covered by predominantly whitish hairs, a few hairs with brownish basal portion; dIII similar in length to IV and longer than dll; digits with long claws (claw of dIII $=5.3 \mathrm{~mm} ; \mathrm{n}=2$ ); tufts of ungual hairs dense and long, extending beyond the claws, whitish colored. Tail shorter than head and body (ranging from 82.4 to $88.1 \%$ of head and body length; $n=3$ ); covered by hairs with length of two to three scales at the basal portion on both surfaces; scales arranged in 13 to 16 transverse rows per centimeter at the base of the tail, between 12 to 14 rows per $\mathrm{cm}$ on the central portion of the tail, and between 15 and 18 rows on the apical centimeter of the tail $(n=2)$; tuft of hairs present at the tail tip (length ranging from 11 to $12 \mathrm{~mm} ; \mathrm{n}=2$ ), whitish colored; dorsal tail color brown, with brown hairs, and ventral tail color white, with whitish hairs; tail strongly bicolor.

Skull robust and elongated (Fig. 53; Table 45). Rostrum very long, with well-developed rostral tube. Nasals anteriorly long, extending beyond the superior incisors, and posteriorly short, not extending beyond the maxil- lary-frontal-lacrimal suture. Premaxillae posteriorly short, ending anteriorly to nasals. Interorbital region divergent posteriorly, with supraorbital crests laterally expanded, less developed in the young specimen. Braincase narrow and elongated. Parietal largely expanded to the lateral surface of braincase. Foramen nasolacrimal ovate and diagonally oriented, above the foramen ethmoid. Postorbital process of the zygoma spinose, formed by the squamosal only. Alisphenoid strut present, foramen buccinators and masticatory confluent, but separated from foramen oval (one specimen), or alisphenoid strut absent, foramen buccinators, masticatory and oval confluent (one specimen). Posterior opening of alisphenoid canal small and visible on lateral surface of skull. Mastoid process long, surpassing the midline of the external auditory meatus. Incisive foramina variable in shape, with convex margins and extremities equally thin (one specimen), or with anterior portion thin and central and posterior portion equally wider (one specimen); incisive foramina septum wide and formed by the premaxillary and maxillary. Mesopterygoid fossa usually "V" shaped, sometimes with the anterior portion slightly rounded, surpassing the center of M2. Sphenopalatine vacuities present but reduced as small openings, posterior to basisphenoid-presphenoid suture. Auditory bullae large. Mandible robust, with ramus deep (Fig. 53). Angular process short and thin, not 


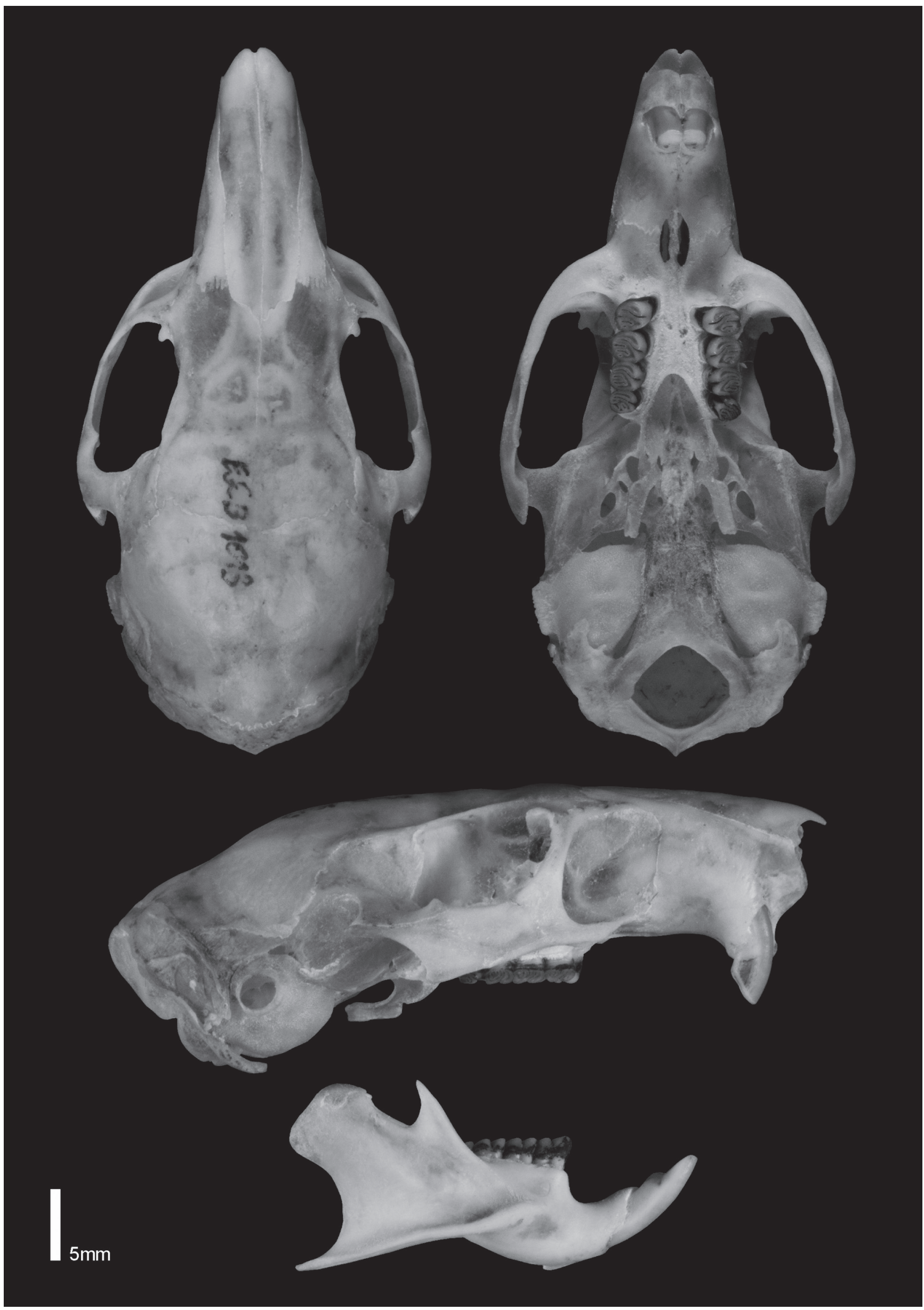

Figure 53. Skull and mandible of Trinomys dimidiatus (EEB 1013; male; GSL = 50.55 mm). Skull on dorsal, ventral, and lateral view, and mandible on lateral view. 
surpassing the condylar process. Superior masseteric ridge reduced and inferior well developed. Ventral mandibular spine absent. Upper incisors orthodont. Maxillary toothrows slightly posteriorly divergent. Upper molars pentalophodont and lower molars tetralophodont.

Remarks: The taxonomy of the genus Trinomys was revised by lack-Ximenes (2005), who recognized 17 species in the genus. Some of these are morphologically and geographically related and were divided into species-groups, among them the dimidiatus group, including $T$. dimidiatus and T. iheringi. According to lack-Ximenes (2005), this group is associated with humid forests of the central-south portion of the Atlantic Forest, with species occurring in the states of São Paulo and Rio de Janeiro. Morphologically, T. dimidiatus and T. iheringi are very similar, being diagnosed mainly by the anatomy of the postorbital process of zygoma, which is formed by the jugal and squamosal bones in T. iheringi and only by the squamosal in T. dimidiatus (lack-Ximenes, 2005; Lara \& Patton, 2000).

To evaluate this and other characteristics that can be used to distinguish the two species, three other specimens of $T$. dimidiatus and 17 specimens of $T$. iheringi were analyzed, from localities in the states of São Paulo and Rio de Janeiro (see additional specimens). This comparative analysis corroborated the differentiation of the postorbital process of the zygoma between the two species, as suggested in previous studies. Furthermore, we observed that the molariforms are wider and have a rounded aspect in $T$. dimidiatus specimens, contrasting with the narrower and less rounded molariforms of T. iheringi. This difference in the width of the molariforms can also be observed in the breadth of PM4 (BPM) in T. dimidiatus and $T$. iheringi, which are presented in the descriptive statistics comparison in Table 45. In the external morphology, specimens of $T$. dimidiatus from EEB, have a small brush at the tail tip, which is predominantly whitish. This characteristic was not observed in specimens of T. iheringi.

Field observations: Only three specimens of $T$. dimidiatus were captured at EEB, with Tomahawk traps and on the same trail during the last expedition to EEB, in December, 2011. As reported for E. russatus, no T. dimidiatus was captured in 2003, 2004 and 2005, during the intensive sampling effort performed in this period. The specimens collected are three females, two adults and one juvenile, none of which showed signs of reproductive activity.

lack-Ximenes (2005) suggested that the distribution of $T$. dimidiatus is associated with the sub-montane forests (between 300 and 1,000 m). At EEB, however, T. dimidiatus was captured above 1,200 m on montane forest.

Additional specimens examined: Trinomys dimidiatus: $\mathrm{n}=3$; Brazil: Rio de Janeiro: Gávea (2258'0.01"S, 4313'59.98"W): female: AMNH 134751; Tijuca

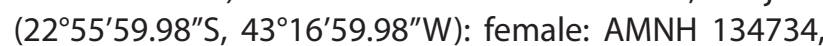
134736. Trinomys iheringi: $\mathrm{n}=17$; Brazil: São Paulo: Casa Grande $\left(23^{\circ} 25^{\prime} 58.79^{\prime \prime} \mathrm{S}, 46^{\circ} 55^{\prime} 58.79^{\prime \prime} \mathrm{W}\right)$ : female: AMNH 245085, 245086; USNM 462133, 462135, 484482, 484484, 484486, 543063, 543064; male: USNM 460561, 462134,
462136, 484483, 484485, 484488, 543065; sex unknown: USNM 460562.

\section{FINAL REMARKS}

The EEB exhibits an outstanding richness of nonvolant small mammals, including many rare and poorly known species of rodents, that are endemic to the Atlantic Forest, such as Abrawayaomys ruschii, Blarinomys breviceps, Drymoreomys albimaculatus, Euryzygomatomys spinosus, Phaenomys ferrugineus, Phyllomys kerri and Rhagomys rufescens, besides the new species of the genus Brucepattersonius here described. The high diversity found at EEB could be explained by a few and complementary facts. First, there has been an extensive sampling effort which was performed for a long period of time. The first six expeditions occurred in 2003 and 2004 and the last expedition in 2011. Even though the sampling effort in 2011 was small, two species that had not been sampled before were captured during that year (noting that the same trap types and same trails used previously were also surveyed in this last expedition); for this reason, we believe that the longterm sampling was determinant to the richness obtained.

Second, the capture success of two expeditions (third and seventh) were especially high. The capture success of the third expedition (8.25\%) is among the highest rates obtained in the Atlantic Forest, comparable to Reserva Florestal do Morro Grande, São Paulo, where Pardini \& Umetsu (2006) obtained a capture success of 7.25\%; with Parque Estadual da Serra do Brigadeiro, Minas Gerais, where Moreira et al. (2009) obtained a capture success of 8.31\%; and with Parque Estadual do Turvo, Rio Grande do Sul, where Melo et al. (2011) obtained a capture success of $7.35 \%$.

Third, the extensive use of pitfall traps was fundamental for trapping rare and even common species. At EEB nine species were captured only with pitfall traps, including some of the rarest rodent species found, such as Drymoreomys albimaculatus, Phaenomys ferrugineus and Phyllomys kerri. Several studies have demonstrated the high efficiency of pitfall traps in capturing nonvolant small mammals in the AF (e.g., Pardini \& Umetsu, 2006; Melo et al., 2011; Bovendorp et al., 2017). According to Pardini \& Umetsu (2006), conventional trap capture (i.e., by Sherman and Tomahawk traps) relies on the animal being attracted to the bait or shelter, whereas pitfall traps capture any passing animal. In other words, pitfall traps are more efficient than conventional traps because they are less selective. The capture ratio of pitfall traps is also enhanced by the plastic sheets fencing the buckets, which direct the animals to them. Voss \& Emmons (1996) and Umetsu et al. (2006) noticed that pitfall traps are effective in capturing juvenile specimens and rare species. It is however important to point out that some species (e.g., Akodon cursor, Euryoryzomys russatus, Trinomys dimidiatus and Philander quica) were collected only by conventional traps, which reinforces the idea that both methods need to be used to ascertain the richness of nonvolant small mammal species in AF localities. 
Finally, the high-quality habitat at EEB was certainly relevant for the richness recorded. Around $75 \%$ of the EEB is maintained as pristine dense forest and the EEB is inserted in the largest continuous of preserved Atlantic Forest (Baitello et al., 1998; Ribeiro et al., 2009), which may have prevented local extinctions. Moreover, the southeastern AF region presents the highest nonvolant small mammal richness in the biome (Costa et al., 2000) and is among the four more diverse areas in South America for rodent species (Amori et al., 2013).

Compared with other surveys in the AF, the species richness of EEB is second only to Parque Nacional do Itatiaia (Rio de Janeiro and Minas Gerais states), where Geise et al. (2004) based on field survey, bibliographic review and museum research, recorded 33 species. In the study of Geise et al. (2004), at least two species that are associated with altitudinal grasslands in the southeastern portion of the AF are included, namely Akodon paranaensis and Thylamys velutinus. Even though this ecosystem is present at EEB, it was not sampled. Besides the Parque Nacional do Itatiaia and EEB, three other localities have the highest number of nonvolant small mammals in the AF: Parque Estadual da Serra do Tabuleiro, Santa Catarina, with 30 species sampled along 20 years and 22 studies compiled by Cherem et al. (2011); Parque Estadual Intervales, São Paulo, with 29 species recognized by Vivo \& Gregorin (2001); and Reserva Florestal do Morro Grande, São Paulo, with 23 species sampled by Pardini \& Umetsu (2006). These results highlight the importance of the southeastern Atlantic Forest in terms of mammal diversity and feature the EEB as one of the richest localities so far sampled in the biome.

Besides documenting this outstanding richness, this monograph had another central component - to assess the intrapopulation morphological variation in rare species, with few available individuals in collections (such as Blarinomys breviceps, Euryzygomatomys spinosus, Juliomys ossitenuis), and also in common species, that are still poorly known in terms of individual variation (such as Akodon montensis, Oligoryzomys nigripes, Sooretamys angouya). We are contributing with important information on variation of diagnostic features which are useful not only for taxonomists working with species limits but also for zoologists and ecologists that need to identify nonvolant small mammals in the field.

We showed that the extracranial course of mandibular nerve is an important characteristic to differentiate Monodelphis iheringi from $M$. scalops. This feature has been previously suggested as important taxonomic trait for didelphid marsupials, but has never been associated to these species (see Voss \& Jansa, 2009; Pavan \& Voss, 2016). For another species of marsupial, Marmosops paulensis, we documented a large variation on the presence and size of palatine fenestrae. Mustrangi \& Patton (1997) suggested that the palatine fenestrae are usually absent in M. paulensis, proposing this as a useful feature to distinguish $M$. paulensis from $M$. incanus. We however suggest that this characteristic should be carefully employed to identify these species.
Regarding rodents, remarks were presented for most species. As an example, we documented a large color variation in Brucepattersonius soricinus, which is strongly associated with ontogenetic development. Chromatic characters have been employed to distinguish species of this genus, specially $B$. soricinus from $B$. igniventris (see Hershkovitz, 1998). We therefore suggest that the external diagnostic features of these species should be reviewed as well as their status as distinct species. Here and in other occasion when larger series become available, researchers were able to define species limits more adequately in this genus, even with the synonymy of some taxa, as B. albinasus (Vilela et al., 2006). Another important contribution to the taxonomy of Brucepattersonius was the description of a new species - B. nebulosus, from the mountain ranges along the Rio Paraíba do Sul valley - that is supported by morphologic and molecular data.

We demonstrated also a great ventral color variation in Oligoryzomys nigripes, in many cases overlapping with the ventral coloration of Oligoryzomys flavescens. However, we noticed that even when these two species present the same ventral coloration, $O$. nigripes always presents a stripe of more intense orange hairs in the pectoral region that stands out, and the transition in the dorsoventral coloration is more evident in O. nigripes. In Juliomys ossitenuis, we quantified an important variation also associated with ontogenetic development. All juveniles and subadults present the interorbital fontanelle, which is absent in all adult specimens. This characteristic was suggested as usually absent in J. ossitenuis by Costa et al. (2007) and, thus, this ontogenetic condition should be highlighted.

The sexual dimorphism was investigated with standardized methods to five species of sigmodontine rodents, one Akodontini (Akodon montensis), one Oryzomyini (Oligoryzomys nigripes), one Wiedomyini (Juliomys ossitenuis), and two incertae sedis (Delomys dorsalis and D. sublineatus). The species that presented more dimorphic variables were Akodon montensis (six variables with significant divergences in subadults) and Juliomys ossitenuis (five variables with significant divergences also in subadults). These results corroborate previous studies with sigmodontine rodents which have found that, when present, sexual dimorphism is more evident in subadults (see Hingst, 1995; Camardella et al., 1998; Libardi, 2013). According to Shine (1989), sexual differences in subadults may result from divergences in niche competition at the onset of sexual maturity - a rapid increase in the cranial dimensions during this phase, more so in males, attenuates the differences with respect to females. Regarding to adults, in each species (except for Juliomys ossitenuis which we were not able to test due the sample size of adults) only one variable was significantly dimorphic. Therefore, we consider that sexual dimorphism is not relevant in the species tested. The results obtained here corroborate those of Abreu-Júnior et al. (2012), which suggested that sexual dimorphism is not an important source of intrapopulation variation in species of the tribe Oryzomyini. We extended this assumption to a broader range of species, including representatives of three tribes plus incertae sedis sigmodontines. It is important to note 
that the identification of sexual divergence in craniometric characters in species of sigmodontine rodents seems to be associated with the type of analysis: as suggested by previous studies, univariate and multivariate analyses, and the proportion of the differences between male and female averages, allow for various interpretations; furthermore, sample size can also strongly influence whether this type of variation is identified or not, as pointed out by Macêdo \& Mares (1987).

\section{ACKNOWLEDGMENTS}

We are thankful to financial support provided by Fundação de Amparo à Pesquisa do Estado de São Paulo (FAPESP; Proc. 2009/16009-1, 2010/14633-7, and 2012/15928-6), Coordenação de Aperfeiçoamento de Pessoal de Ensino Superior (CAPES), and Conselho Nacional de Desenvolvimento Científico e Tecnológico (CNPq). We are also grateful to Universidade de São Paulo and the Programa de Pós-Graduação Interunidades em Ecologia Aplicada (ESALQ/CENA) for the graduate fellowship and logistic support. We are much indebted to researchers and staff of MZUSP and EEB, especially Hussam Zaher, Mario de Vivo, Luis Fábio Silveira and José Roberto Alves Suarez, for inviting us to join some expeditions (2003 and 2011) to the EEB, for carrying out the field work expeditions and for making available the specimens, fieldwork notes and catalogs. We are indebted to the curators and staff of all museums and collections studied. We also would like to acknowledge Sharon A. Jansa for providing financial and technical support for molecular analyses and to Lorissa Fujishin for helping on molecular procedures. We are also thankful to James $L$. Patton, Dayse Dias and Yuri L.R. Leite for kindly providing valuable CYTB sequences. Our thanks to Gustavo S. Libardi for drawing the skulls. Guillermo D'Elía, Pablo R. Gonçalves and Guilherme S.T. Garbino provided important and valuable comments on earlier drafts of this manuscript: they helped us to improve it and any problems that may remain are our own.

\section{REFERENCES}

Abreu-Júnior, E.F.; Brennand, P.G.G.; Chiquito, E.A.; Jorge-Rodrigues, C.R.; Libardi, G.S.; Prado, J.R. \& Percequillo, A.R. 2012. Dimorfismo sexual na tribo Oryzomyini. In: Freitas, T.R.O. \& Vieira, E.M. (Eds.). Mamíferos do Brasil: genética, sistemática, ecologia e conservação. Rio de Janeiro, Sociedade Brasileira de Mastozoologia. p. 115-134.

Abreu-Júnior, E.F.; Percequillo, A.R.; Geise, L.; Leite, Y.L.R. \& Loss, A.C. 2018. Unveiling the identity of Kerr's Atlantic tree rat, Phyllomys kerri (Rodentia, Echimyidae). Mammalian Biology, 91: 57-70.

Agrellos, R.; Bonvicino, C.R.; Rosa, E.S.T.; Marques, A.A.R.; D'Andrea, P.S.D. \& Weksler, M. 2012. The taxonomic status of the Castelo dos Sonhos Hantavirus reservoir, Oligoryzomys utiaritensis Allen 1916 (Rodentia: (ricetidae: Sigmodontinae). Zootaxa, 3220: 1-28.

Amori, G.; Chiozza, F.; Patterson, B.; Rondinini, C.; Schipper, J. \& Luiselli, L. 2013. Species richness and distribution of Neotropical rodents, with conservation implications. Mammalia, 77: 1-19. DOI
Baitello, J.B.; Aguiar, 0.T.; Rocha, F.T. \& Carvalho, J.L. 1998. Plano de Manejo das Unidades de Conservação: Estação Ecológica de Bananal - Vegetação. PPMA/IF/KFW, São Paulo.

Bonvicino, C.R. 1994. Especiação do rato d'água Nectomys: abordagem cariológica, morfológica e geográfica. Doctoral Thesis. Rio de Janeiro, Universidade Federal do Rio de Janeiro.

Bonvicino, C.R. \& Weksler, M. 1998. A new species of Oligoryzomys (Rodentia, Sigmodontinae) from (entral Brazil. Zeitschrift für Säugetierkunde, 63: 90-103.

Bonvicino, C.R.; Oliveira, J.A.; D'Andrea, P.S. \& Carvalho, R.W.K. 2001. The endemic Atlantic Forest Rodent Phaenomys ferrugineus (Thomas, 1894) (Sigmodontinae): new data on its morphology and karyology. Boletim do Museu Nacional, 467: 1-12.

Bonvicino, C.R.; Penna-Firme, V. \& Seuánez, H.N. 1998. The karyotype of Brucepattersonius griserufescens Hershkovitz. 1998 (Rodentia, Sigmodontinae) with comments on distribution and taxonomy. Zeitschrift für Säugetierkunde, 63(6): 329-335.

Bovendorp, R.S.; Villar, N.; Abreu-Júnior, E.F.; Bello, C.; Regolin, A.L.; Percequillo, A.R. \& Galetti, M. 2017. Atlantic Small-Mammal: a dataset of communities of rodents and marsupials of the Atlantic Forests of South America. Ecology, 98(8): 2226.

Brandt, R.S. \& Pessôa, L.M. 1994. Intrapopulational variability in cranial characters of Oryzomys subflavus (Wagner, 1842), in northeastern Brazil. Zoologischer Anzeiger, 233: 45-55.

Câmara, I.G. 2005. Breve história da conservação da Mata Atlântica. In: Galindo-Leal, C. \& Câmara, I.G. (Eds.). Mata Atlântica: biodiversidade, ameaças e perspectivas. Belo Horizonte, Fundação SOS Mata Atlântica e Conservação Internacional. p. 31-42.

Camardella, A.R.; Pessôa, L.M. \& Oliveira, J.A. 1998. Sexual dimorphism and age variability in cranial characters of Oryzomys subflavus (Wagner, 1842) (Rodentia: Sigmodontinae) from northeastern Brazil. Bonner Zoologische Beiträge, 48: 9-18.

Campanili, M. \& Prochnow, M. 2006. Mata Atlântica - uma rede pela floresta. Brasília, RMA.

Carleton, M.D. 1980. Phylogenetic relationships in Neotomine-Peromyscine rodents (Muroidea) and a reappraisal of the dichotomy within New World Cricetinae. Miscellaneous Publications, Museum of Zoology, University of Michigan, 157: 1-146.

Carleton, M.D. \& Musser, G.G. 1989. Systematic studies of oryzomyine rodents (Muridae, Sigmodontidae): a synopsis of Microryzomys. Bulletin of the American Museum of Natural History, 191: 1-83.

Castro, A.G. 2001. Levantamento florístico de um trecho de Mata Atlântica na Estação Ecológica de Bananal, Serra da Bocaina, Bananal, São Paulo. Masters Dissertation. Universidade Federal Rural do Rio de Janeiro, Seropédica.

Cerboncini, R.A.S.; Zanata, T.B.; Cunha, W.L.; Rorato, A.M.; Calefi, A.S.; Sbeghen, M.R.; Macagnan, R.; Abreu, K.C.; Ono, M.A. \& Passos, F.C. 2014. Distribution extension of Abrawayaomys ruschii Cunha and Cruz, 1979 (Rodentia: (ricetidae) with the first records in the state of Paraná, southern Brazil. Check List, 10(3): 660-662. D0I

Cherem, J.J.; Graipel, M.E.; Tortato, M.; Althoff, S.; Brüggemann, F.; Matos, J.; Voltolini, J.C.; Freitas, R.; Illenseer, R.; Hoffmann, F.; Ghizoni-Jr., I.R.; Bevilacqua, A.; Reinicke, R.; Salvador, C.H.; Filippini, A.; Furnari, N.; Abati, K.; Moraes, M.; Moreira, T.; Oliveira-Santos, L.G.R.; Kuhnen, V.; Maccarini, T.; Goulart, F.; Mozerle, H.; Fantacini, F.; Dias, D.; Penedo-Ferreira, R.; Vieira, B.P. \& Simões-Lopes, P.C. 2011. Mastofauna terrestre do Parque Estadual da Serra do Tabuleiro, Estado de Santa Catarina, sul do Brasil. Biotemas, 24(3): 73-84. D0I

Chiquito, E.A.; D'Elía, G. \& Percequillo, A.R. 2014. Taxonomic review of genus Sooretamys Weksler, Percequillo \& Voss (Rodentia: Cricetidae: 
Sigmodontinae): an integrative approach. Zoological Journal of the Linnean Society, 171: 842-877. DOI

Christoff, A.U.; Fagundes, V.; Sbalquero, I.J.; Mattevi, M.S. \& YonenagaYassuda, Y. 2000. Description of a new species of Akodon (Rodentia: Sigmodontinae) from southern Brazil. Journal of Mammalogy, 81: 838-851. DO

Christoff, A.U.; Vieira, E.M.; Oliveira, L.R.; Gonçalves, J.W.; Valiati, V.H. \& Tomasi, P.S. 2016. A new species of Juliomys (Rodentia, Cricetidae, Sigmodontinae) from the Atlantic Forest of Southern Brazil. Journal of Mammalogy, 97(5): 1469-1482. DOI

Cirignoli, S.; Galliari, C.A.; Pardiñas, U.F.J. \& Podestá, D.H. 2011. Mamíferos de la Reserva Valle del Cuña Pirú, Misiones, Argentina. Mastozoologia Neotropical, 18: 25-43.

Costa, B.M.A.; Geise, L.; Pereira, L.G. \& Costa, L.P. 2011. Phylogeography of Rhipidomys (Rodentia: Cricetidae: Sigmodontinae) and description of two new species from southeastern Brazil. Journal of Mammalogy, 92(5): 945-962. DOI

Costa, L.P.; Leite, Y.L.R.; Fonseca, G.A.B. \& Fonseca, M.T. 2000. Biogeography of South American forest mammals: endemism and diversity in the Atlantic forest. Biotropica, 32(4b): 872-881. D0I

Costa, L.P.; Pavan, S.E.; Leite, Y.L.R. \& Fagundes, V. 2007. A new species of Juliomys (Mammalia, Rodentia, Cricetidae) from the Atlantic Forest of southeastern Brazil. Zootaxa, 1463: 21-37.

da Silva, J.M.C. \& Casteletti, C.H.M. 2003. Status of the biodiversity of the Atlantic Forest of Brazil. In: Galindo-Leal, C. \& Câmara, I.G. (Eds.). The Atlantic forest of South America: biodiversity status, threats, and outlook. Washington, CABS and Island Press. p. 43-59.

Darriba, D.; Taboada, G.L.; Doallo, R. \& Posada, D. 2012. jModelTest 2: more models, new heuristics and parallel computing. Nature Methods, 9(8): 772.

Dias, D. 2016. Sistemática molecular, biogeografia e diversificação de Brucepattersonius (Rodentia: Sigmodontinae). Masters Dissertation. Universidade Federal do Espírito Santo, Vitória.

Edgar, R.C. 2004. MUSCLE: Multiple sequence alignment with high accuracy and high throughput. Nucleic Acids Research, 32: 1792-1797.

Figueiredo, M.S.L.; Barros, C.S.; Delciellos, A.C.; Guerra, E.B.; Cordeiro-Estrela, P.; Kajin, M.; Alvarez, M.R.; Asfora, P.H.; Astúa, D.; Bergallo, H.G.; Cerqueira, R.; Geise, L.; Gentile, R.; Grelle, C.E.V.; lack-Ximenes, G.E.; Oliveira, L.C.; Weksler, M. \& Vieira, M.V. 2017. Abundance of small mammals in the Atlantic Forest (ASMAF): a data set for analyzing tropical community patterns. Ecology, 98(11): 2981. D0l

Fundação para a Conservação e a Produção Florestal do Estado de São Paulo (Fundação Florestal). 2013. Estação Ecológica de Bananal. Available at: http://www.ambiente.sp.gov.br/e-e-bananal. Access in: 14/08/2013.

Fundação SOS Mata Atlântica \& Instituto Nacional de Pesquisas Espaciais (INPE). 2013. Atlas dos remanescentes florestais da mata atlântica, período 2011-2012. São Paulo, Fundação SOS Mata Atlântica, Instituto Nacional de Pesquisas Espaciais.

Geise, L. 2012. Akodon cursor (Rodentia: Cricetidae). Mammalian Species, 44(893): 33-43. DOI

Geise, L.; Bergallo, H.G.; Esbérard, C.E.L.; Rocha, C.F.D. \& Van Sluys, M. 2008. The karyotype of Blarinomys breviceps (Mammalia: Rodentia: Cricetidae) with comments on its morphology and some ecological notes. Zootaxa, 1907: 47-60.

Geise, L.; Moraes, D.A. \& Silva, H.S. 2005. Morphometric differentiation and distributional notes of three species of Akodon (Muridae, Sigmodontinae, Akodontini) in the Atlantic coastal area of Brazil. Arquivos do Museu Nacional, 63(1): 63-74.

Geise, L.; Pereira, L.G.; Bossi, D.E. \& Bergallo, H.G. 2004. Patterns of elevational distribution and richness of nonvolant mammals in Itatiaia National Park and surroundings, in Southeastern Brazil. Brazilian Journal of Biology, 64: 1-15. DOI

Gonçalves, P.R. \& Oliveira, J.A. 2004. Morphological and genetic variation between two sympatric forms of Oxymycterus (Rodentia: Sigmodontinae): an evaluation of hypotheses of differentiation within the genus. Journal of Mammalogy, 85(1): 148-161. DOI

Gonçalves, P.R. \& Oliveira, J.A. 2014. An integrative appraisal of the diversification in the Atlantic forest genus Delomys (Rodentia: Cricetidae: Sigmodontinae) with the description of a new species. Zootaxa, 3760(1): 1-38. DOI

Gonçalves, P.R.; Myers, P.; Vilela, J.F. \& Oliveira, J.A. 2007. Systematics of the species of the genus Akodon (Rodentia: Sigmodontinae) in Southeastern Brazil and implications for the biogeography of the campos de altitude. Miscellaneous Publications, Museum of Zoology, University of Michigan, 197: 1-24.

González, E.M. 2000. Un nuevo genero de roedor sigmodontino de Argentina y Brasil (Mammalia: Rodentia: Sigmodoninae). Comunicaciones Zoológicas del Museo de Historia Natural de Montevideo, 195(12): 1-12.

Guindon, S. \& Gascuel, 0. 2003. A simple, fast and accurate method to estimate large phylogenies by maximum-likelihood. Systematic Biology, 52: 696-704.

Hershkovitz, P. 1944. Systematic review of the Neotropicl water rats of the genus Nectomys (Cricetinae). Miscellaneous Publications, Museum of Zoology, University of Michigan, 58: 1-101.

Hershkovitz, P. 1962. Evolution of Neotropical cricetine rodents (Muridae), with special reference to the phyllotine group. Fieldiana, Zoology, 46: 1-524. DOI

Hershkovitz, P. 1998. Report on some Sigmodontinae rodents collected in southeastern Brazil with descriptions of a new genus and six new species. Bonner Zoologische Beiträge, 47: 193-256.

Hingst, E.D. 1995. Reprodução, crescimento e desenvolvimento em Bolomys lasiurus (Rodentia, Sigmodontinae). Masters Dissertation. Rio de Janeiro, Universidade Federal do Rio de Janeiro.

lack-Ximenes, G.E. 2005. Revisão de Trinomys Thomas, 1921 (Rodentia: Echimyidae). Doctoral Thesis. São Paulo, Universidade de São Paulo.

Jansa, S.A.; Goodman, S.M. \& Tucker, P.K. 1999. Molecular phylogeny and biogeography of the native rodents of Madagascar (Muridae: Nesomyidae): a test of the single origin hypothesis. Cladistics, 15: 253-270.

Johnson, R.A. \& Wichern, D.W. 1999. Applied multivariate statistical analysis. New Jersey, Upper Saddle River.

Jung, D.M.H. 2009. Filogenia e Filogeografia de Roedores Sigmodontíneos do Rio Grande do Sul. 2009. Masters Dissertation. São Leopoldo, Universidade do Vale do Rio do Sino.

Lara, M.C. \& Patton, J.L. 2000. Evolutionary diversification of spiny rats (genus Trinomys, Rodentia: Echimyidae) in the Atlantic forest of Brazil. Zoological Journal of the Linnean Society, 130: 661-686. D0I

Leite, Y.L.R. 2003. Evolution and systematics of the Atlantic Tree Rats, Genus Phyllomys (Rodentia, Echimyidae), with description of two new species. University of California Publications, 132: 1-135. D01

Libardi, G.S. 2013. Variação não-geográfica em Necromys lasiurus (Lund, 1840) (Cricetidae: Sigmodontinae) no Brasil. Masters Dissertation. Piracicaba, SP, ESALQ/Universidade de São Paulo.

Lóss, S.; Costa, L.P. \& Leite, Y.L.R. 2011. Geographic variation, phylogeny and systematic status of Gracilinanus microtarsus (Mammalia: Didelphimorphia: Didelphidae). Zootaxa, 2761: 1-33.

Macêdo, R.H. \& Mares, M.A. 1987. Geographic variation in the South American cricetine rodent Bolomys lasiurus. Journal of Mammalogy, 68(3): 578-594. DOI

Machado, L.F.; Paresque, R. \& Christoff, A.U. 2011. Anatomia comparada e morfometria de Oligoryzomys nigripes e 0 . flavescens (Rodentia, 
Sigmodontinae) no Rio Grande do Sul, Brasil. Papéis Avulsos de Zoologia, 51(3): 29-47. DOI

Manly, B.F.J. 2005. Multivariate statistical methods: A Primer. New York, Chapman \& Hall/CRC.

Mares, M.A. \& Braun, J.K. 2000. Three new species of Brucepattersonius (Rodentia:Sigmodontinae) from Misiones Province, Argentina. Occasional Papers of the Sam Noble Oklahoma Museum of Natural History, 9: 1-13.

Melo, G.L.; Sponchiado, J.; Machado, A.F. \& Cáceres, N.C. 2011. Smallmammal community structure in a South American deciduous Atlantic Forest. Community Ecology, 12: 58-66. DOI

Moreira, J.C.; Manduca, E.G.; Gonçalves, P.R.; Morais Jr., M.M.; Pereira, R.F.; Lessa, G. \& Dergam, J.A. 2009. Small mammals from Serra do Brigadeiro State Park, Minas Gerais, southeastern Brazil: species composition and elevational distribution. Arquivos do Museu Nacional, 67(1/2): 103-118.

Musser, G.G.; Carleton, M.D.; Brothers, E.M. \& Gardner, A.L. 1998. Systematic studies of oryzomyine rodents (Muridae, Sigmodontinae): diagnoses and distributions of species formerly assigned to Oryzomys "capito". Bulletin of the American Museum of Natural History, 236: 1-376.

Mustrangi, M.A. \& Patton, J.L. 1997. Phylogeography and systematics of the slender mouse opossum Marmosops (Marsupialia, Didelphidae). University of California Publications in Zoology, 130: 1-86.

Myers, N.; Mittermeier, R.A.; Mittermeier, C.G.; Fonseca, G.A.B. \& Kent, J. 2000. Biodiversity hotspots for conservation priorities. Nature, 403: 853-858. D01

Myers, P. \& Carleton, M.D. 1981. The Species of Oryzomys (Oligoryzomys) in Paraguay and the Identity of Azara's "Rat sixieme ou Rat a Tarse Noir". Miscellaneous Publications Museum of Zoology, University of Michigan, 161: 1-41.

Myers, P.; Patton, J.L. \& Smith, M.F. 1990. A review of the boliviensis group of Akodon (Muridae: Sigmodontinae), with emphasis on Peru and Bolivia. Miscellaneous Publications, Museum of Zoology, University of Michigan, 177: 1-104.

Neff, N.A. \& Marcus, L.F. 1980. A survey of multivariate methods for systematics. New York, American Museum of Natural History.

Oliveira, J.A. \& Bonvicino, C.R. 2002. A new species of sigmodontine rodent from the Atlantic Forest of eastern Brazil. Acta Theriologica, 47: 307-322. DOI

Oliveira, J.A. \& Gonçalves, P.R. 2015. Genus Oxymycterus Waterhouse, 1837. In: Patton, J.L.; Pardiñas, U.F.J. \& D'Elía, G. (Eds.). Mammals of South America, Vol. 2, Rodents. Chicago, The University of Chicago. p. 247-268.

Paglia, A.P.; Fonseca, G.A.B. da; Rylands, A.B.; Herrmann, G.; Aguiar, L.M.S.; Chiarello, A.G.; Leite, Y.L.R.; Costa, L.P.; Siciliano, S.; Kierulff, M.C.M.; Mendes, S.L.; Tavares, V.C.; Mittermeier, R.A. \& Patton, J.L. 2012. Annotated checklist of Brazilian Mammals. 2. ed. Occasional Papers in Conservation Biology, 6: 1-76.

Pardiñas, U.F.J.; Teta, P. \& D'Elía, G. 2009. Taxonomy and distribution of Abrawayaomys (Rodentia: Cricetidae), an Atlantic Forest endemic with the description of a new species. Zootaxa, 2128: 39-60.

Pardiñas, U.F.J.; Teta, P.; Alvarado-Serrano, D.F.; Geise, L.; Jayat, J.P.; Ortiz, P.E.; Gonçalves, P.R. \& D'Elía, G. 2015. Genus Akodon Meyen, 1833. In: Patton, J.L.; Pardiñas, U.F.J. \& D'Elía, G. (Eds.). Mammals of South America, Vol. 2, Rodents. Chicago, The University of Chicago Press. p. 144-204.

Pardini, R. \& Umetsu, F. 2006. Pequenos mamíferos não-voadores da Reserva Florestal do Morro Grande: distribuição das espécies e da diversidade em uma área de Mata Atlântica. Biota Neotropica, 6(2): 1-22. DOI

Paresque, R. 2010. Diversificação das espécies do gênero Oligoryzomys Bangs, 1900 (Rodentia, Cricetidae) na região neotropical. Doctoral Thesis. São Paulo, Universidade de São Paulo. DOI

Passamani, M.; Cerboncini, R.A.S. \& Oliveira, J.E. 2011. Distribution extension of Phaenomys ferrugineus (Thomas, 1894), and new data on
Abrawayaomys ruschii Cunha and Cruz, 1979 and Rhagomys rufescens (Thomas, 1886), three rare species of rodents (Rodentia: Cricetidae) in Minas Gerais, Brazil. Check List, 7(6): 827-831. D01

Patton, J.L.; da Silva, M.N.F. \& Malcolm, J.R. 2000. Mammals of the Rio Juruá and the evolutionary and ecological diversification of Amazonia. Bulletin of the American Museum of Natural History, 244: 1-306. DOI

Pavan, S.E. 2015. A new species of Monodelphis (Didelphimorphia: Didelphidae) from the Brazilian Atlantic Forest. American Museum Novitates, 3832: 1-15.

Pavan, S.E. 2019. A revision of the Monodelphis glirina group (Didelphidae: Marmosini), with a description of a new species from Roraima, Brazil. Journal of Mammalogy, 100(1): 103-117.

Pavan, S.E. \& Leite, Y.L.R. 2011. Morphological diagnosis and geographic distribution of Atlantic Forest red-rumped mice of the genus Juliomys (Rodentia: Sigmodontinae). Zoologia, 28: 663-672. D01

Pavan, S.E. \& Voss, R.S. 2016. A revised subgeneric classification of shorttailed opossums (Didelphidae: Monodelphis). American Museum Novitates, 3868: 1-44.

Pavan, S.E.; Jansa, S.A. \& Voss, R.S. 2014. Molecular phylogeny of short-tailed opossums (Didelphidae: Monodelphis): Taxonomic implications and tests of evolutionary hypotheses. Molecular Phylogenetics and Evolution, 79: 199-214.

Percequillo, A.R. 1998. Sistemática de Oryzomys Baird, 1858 do leste do Brasil (Muroidea, Sigmodontinae). Masters Dissertation. São Paulo, Universidade de São Paulo.

Percequillo, A.R. 2003. Sistemática de Oryzomys Baird, 1858: definição dos grupos de espécies erevisão do grupo albigularis (Rodentia, Sigmodontinae). Doctoral Thesis. São Paulo, Universidade de São Paulo.

Percequillo, A.R. 2015a. Genus Euryoryzomys Weksler, Percequillo, and Voss, 2006. In: Patton, J.L.; Pardiñas, U.F.J. \& D'Elía, G. (Eds.). Mammals of South America, Vol. 2, Rodents. Chicago, The University of Chicago Press. p. 312-321.

Percequillo, A.R. 2015b. Genus Phaenomys Thomas, 1917. In: Patton, J.L.; Pardiñas, U.F.J. \& D'Elía, G. (Eds.). Mammals of South America, Vol. 2, Rodents. Chicago, The University of Chicago Press. p. 101-103.

Percequillo, A.R.; Braga, C.A.C.; Brandão, M.V.; Abreu-Júnior, E.F.; GualdaBarros, J.; Lessa, G.M.; Pires, M.R.S. \& Hingst-Zaher, E. 2017. The genus Abrawayaomys Cunha and Cruz, 1979 (Rodentia: Cricetidae: Sigmodontinae): geographic variation and species definition. Journal of Mammalogy, 98: 438-455.

Percequillo, A.R.; Gonçalves, P.R. \& Oliveira, J.A. 2004. The rediscovery of Rhagomys rufescens (Thomas, 1886), with a morphological redescription and comments on its systematic relationships based on morphological and molecular (cytochrome b) characters. Mammalian Biology -Zeitschrift für Säugetierkunde, 69(4): 238-257. DOI

Percequillo, A.R.; Weksler, M. \& Costa, L.P. 2011. A new genus and species of rodent from the Brazilian Atlantic Forest (Rodentia: Cricetidae: Sigmodontinae: Oryzomyini), with comments on oryzomyine biogeography. Zoological Journal of the Linnean Society, 161:357-390. D0I

Pereira, J.; Teta, P.; Fracassi, N.; Johnson, A. \& Moreyra, P. 2005. Sigmodontinos (Rodentia, Cricetidae) de la Reserva de Vida Silvestre Urugua-í (Provincia de Misiones, Argentina), con la confirmación de la presencia de "Akodon" serrensis en la Argentina. Mastozoologia Neotropical, 12: 83-89.

Pinheiro, P.S.; Hartmann, P.A. \& Geise, L. 2004. New record of Rhagomys rufescens (Thomas, 1886) (Rodentia: Muridae: Sigmodontinae) in the Atlantic Forest of southeastern Brazil. Zootaxa, 431: 1-11.

Prado, J.R. \& Percequillo, A.R. 2011. Ontogenetic and sexual variation in cranial characters of Aegialomys xanthaeolus (Thomas, 1894) (Cricetidae: Sigmodontinae) from Ecuador and Peru. Papéis Avulsos de Zoologia, 51(9): 155-177. DOI 
Rambaut, A. 2012. FigTree v1.4. Available at: http://tree.bio.ed.ac.uk/ software/figtree. Access in: 27/07/2013.

Rambaut, A. \& Drummond, A.J. 2007. Tracer v1.5. Available at: http://beast. bio.ed.ac.uk/Tracer. Access in: 2/05/2013.

Ribeiro, M.C.; Metzger, J.P.; Martensen, A.C.; Ponzoni, F.J. \& Hirota, M.M. 2009. The Brazilian Atlantic Forest: How much is left, and how is the remaining forest distributed? Implications for conservation. Biological Conservation, 142: 1141-1153. DOI

Ronquist, F.; Teslenko, M.; Van Der Mark, P.; Ayres, D.; Darling, A.; Höhna, S.; Larget, B.; Liu, L.; Suchard, M.A. \& Huelsenbeck, J.P. 2012 MrBayes 3.2: Efficient Bayesian phylogenetic inference and model choice across a large model space. Systematic Biology, 61(3): 539-542.

Rossi, R.V.; Voss, R.S. \& Lunde, D.P. 2010. A revision of the didelphid marsupial genus Marmosa. Part 1. The species in Tate's "Mexicana" and "Mitis" sections and other closely related forms. Bulletin of the American Museum of Natural History, 334: 1-83. D0I

Shine, R. 1989. Sexual size dimorphism and male combat in snakes. Oecologia, 33: 269-277. DOI

Sikes, R.S. \& The Animal Care and Use Committee. 2016. 2016 Guidelines of the American Society of Mammalogists for the use of wild mammals in research and education. Journal of Mammalogy, 97: 663-688.

Smith, M.F. \& Patton, J.L. 1993. The diversification of South American murid rodents: evidence from mitochondrial DNA sequence data for the akodontine tribe. Biological Journal of the Linnean Society, 50(3): 149-177.

Sokal, R.R. \& Rohlf, F.J. 1981. Biometry: the principles and practice of statistics in biological research. 2. ed. New York, Ed. W.H. Freeman. 859p.

SPSS Inc. 2008. Statistical Package for the Social Sciences. Statistics for Windows, version 17.0. Chicago, Illinois.

Stamatakis, A. 2006. RAxML-VI-HPC: Maximum Likelihood-based Phylogenetic Analyses with Thousands of Taxa and Mixed Models. Bioinformatics, 22(21): 2688-2690.

Steiner-Souza, F.; Cordeiro-Estrela, P.; Percequillo, A.R.; Testoni, A.F. \& Althoff, S.L. 2008. New records of Rhagomys rufescens (Rodentia: Sigmodontinae) in the Atlantic forest of Brazil. Zootaxa, 1824: 28-34.

Svartman, M. \& Cardoso de Almeida, E.J. 1993. The karyotype of Oxymycterus sp. (Cricetidae, Rodentia) from Central Brazil. Experientia, 49: 718-720.

Tamura, K.; Stecher, G.; Peterson, D.; Filipski, A. \& Kumar, S. 2013. MEGA6: Molecular Evolutionary Genetics Analysis Version 6.0. Molecular Biology and Evolution, 30: 2725-2729.

Tavares, W.C.; Pessôa, L.M. \& Gonçalves, P.R. 2011. New species of Cerradomys from coastal sandy plains of southeastern Brazil (Cricetidae: Sigmodontinae). Journal of Mammalogy, 92(3): 645-658. D01

Teta, P.; Jayat, J.P.; Ortiz, P.E. \& D'Elía, G. 2013. The taxonomic status of Oligoryzomys brendae Massoia, 1998 (Rodentia, Cricetidae), with comments on the availability of this name. Zootaxa, 3641(4): 433-447.D01

Thomas, 0. 1886. Description of a new Brazilian species of Hesperomys. Annals and Magazine of Natural History, Ser. 5, 17: 250-251.

Thomas, 0. 1894. Descriptions of some new Neotropical Muridae. Annals and Magazine of Natural History, Ser. 5, 14: 346-366.

Thomas, 0. 1917. On the arrangement of the South American rats allied to Oryzomys and Rhipidomys. Annals and Magazine of Natural History, Ser. 8, 20: 192-198.

Tribe, C.J. 2015. Genus Rhipidomys Tschudi, 1845. In: Patton, J.L.; Pardiñas, U.F.J. \& D'Elía, G. (Eds.). Mammals of South America, Vol. 2, Rodents. Chicago, The University of Chicago Press. p. 583-617.

Umetsu, F.; Naxara, L. \& Pardini, R. 2006. Evaluating the efficiency of pitfall traps for sampling small mammals in the Neotropics. Journal of Mammalogy, 87(4): 757-765. DOI

Vanzolini, P.E. 1993. Métodos estatísticos elementares em sistemática zoológica. São Paulo, Hucitec.
Vaz, S.M. 2000. Sobre a distribuição geográfica de Phaenomys ferrugineus (Thomas) (Rodentia, Muridae). Revista Brasileira de Zoologia, 17: 183-186. DOI Veloso, H.P.; Rangel Filho, A.L.R. \& Lima, J.C.A. 1991. Classificação da vegetação brasileira adaptada a um sistema universal. Rio de Janeiro, IBGE, Departamento de Recursos Naturais e Ambientais.

Ventura, J.; Lopez-Fuster, M.J.; Salazar, M. \& Perez-Hernandez, R. 2000. Morphometric analysis of some Venezuelan Akodontine rodents. Netherlands Journal of Zoology, 50: 487-501. D0I

Vilela, J.F. 2005. Filogenia molecular do gênero Brucepattersonius (Rodentia: Sigmodontinae) com uma análise filogeográfica de B. griserufescens. Masters Dissertation. Rio de Janeiro, Museu Nacional, Universidade Federal do Rio de Janeiro.

Vilela, J.F.; Gonçalves, P.R. \& Oliveira, J.A. 2015. Genus Brucepattersonius Hershkovitz, 1998. In: Patton, J.L.; Pardiñas, U.F.J. \& D'Elía, G. (Eds.). Mammals of South America, Vol. 2, Rodents. Chicago, The University of Chicago Press. p. 211-219.

Vilela, J.F.; Oliveira, J.A. \& Bonvicino, C.R. 2006. Taxonomic status of Brucepattersonius albinasus (Rodentia: Sigmodontinae). Zootaxa, 1199:61-68.

Vivo, M. \& Gregorin, R. 2001. Mamíferos. In: Leonel, C. (Ed.). Intervales. São Paulo, Fundação para a Conservação e a Produção Florestal do Estado de São Paulo. p. 116-123.

Voss, R.S. 1988. Systematics and ecology of Ichthyomyine rodents (Muroidea): patterns of morphological evolution in a small adaptive radiation. Bulletin of the American Museum of Natural History, 188: 259-493.

Voss, R.S. 1991. An introduction to the Neotropical muroid rodent genus Zygodontomys. Bulletin of the American Museum of Natural History, 210: 1-113.

Voss, R.S. 1993. A revision of the Brazilian muroid rodent genus Delomys: with remarks on "thomasomyine" characters. American Museum Novitiates, 3073: 1-44.

Voss, R.S. \& Emmons, L.H. 1996. Mammalian diversity in Neotropical lowland rainforests: a preliminary assessment. Bulletin of the American Museum of Natural History, 230: 1-115.

Voss, R.S. \& Jansa, S.A. 2003. Phylogenetic studies on didelphid marsupials II. Nonmolecular data and new IRBP sequences: separate and combined analyses of didelphine relationships with denser taxon sampling. Bulletin of the American Museum of Natural History, 276: 1-82. D01

Voss, R.S. \& Jansa, S.A. 2009. Phylogenetic relationships and classification of Didelphid marsupials, an extant radiation of new world metatherian mammals. Bulletin of the American Museum of Natural History, 322: 1-177. D01

Voss, R.S.; Lunde, D.P. \& Simmons, N.B. 2001. The Mammals of Paracou, French Guiana: A Neotropical Lowland Fauna. Part 2. Nonvolant Species. Bulletin of the American Museum of Natural History, 263: 1-236. D0I

Voss, R.S.; Yensen, E. \& Tarifa, T. 2004. An introduction to Marmosops (Marsupialia: Didelphidae), with the description of a new species from Bolivia and notes on the taxonomy and distribution of other Bolivian congeners. American Museum Novitates, 3466: 1-40. D01

Weksler, M. 2006. Phylogenetic relationships of Oryzomyine rodents (Muroidea: Sigmodontinae): separate and combined analyses of morphological and molecular data. Bulletin of the American Museum of Natural History, 296: 1-149. D0I

Weksler, M. \& Bonvicino, C.R. 2005. Taxonomy of pigmy rice rats genus Oligoryzomys Bangs, 1900 (Rodentia, Sigmodontinae) of the Brazilian Cerrado, with the description of two new species. Arquivos do Museu Nacional, 63(1): 113-130.

Weksler, M. \& Bonvicino, C.R. 2015. Genus Oligoryzomys Bangs, 1900. In: Patton, J.L.; Pardiñas, U.F.J. \& D'Elía, G. (Eds.). Mammals of South America, Vol. 2, Rodents. Chicago, The University of Chicago Press. p. 417-437.

Weksler, M.; Percequillo, A.R. \& Voss, R.S. 2006. Ten new genera of Oryzomyine Rodents (Cricetidae: Sigmodontinae). American Museum Novitates, 3537: 1-29. DOI 